\title{
SCANNING THERMAL LITHOGRAPHY FOR NANOPATTERNING OF POLYMERS
}

Transient Heat Transport and Thermal Chemical Functionalization Across the Length Scales

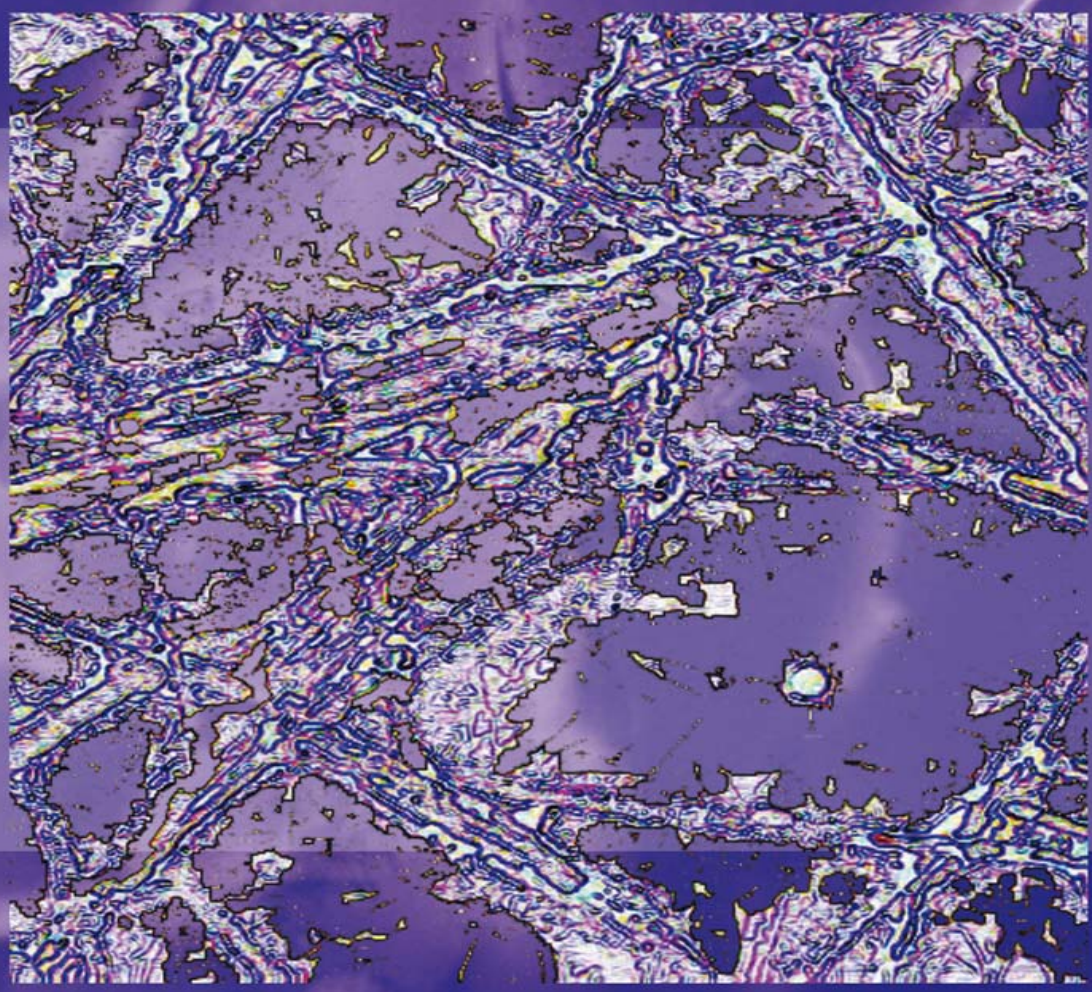


Scanning Thermal Lithography for Nanopatterning of Polymers

Transient Heat Transport and Thermal Chemical Functionalization Across the Length Scales 
This research was financially supported by the MESA+ Institute for Nanotechnology of the University of Twente and NanoNed, the nanotechnology program of the Dutch Ministry of Economic Affairs (grant TPC.6940).

Scanning Thermal Lithography for Nanopatterning of Polymers: Transient Heat Transport and Thermal Chemical Functionalization Across the Length Scales

J. Duvigneau

Ph.D. Thesis

University of Twente, Enschede, The Netherlands

(C) Joost Duvigneau 2011

ISBN: 978-90-365-3131-3

No part of this work may be reproduced by print, photocopy or any other means without the permission of the publisher. 


\title{
SCANNING THERMAL LITHOGRAPHY FOR NANOPATTERNING OF POLYMERS
}

\section{TRANSIENT HEAT TRANSPORT AND THERMAL CHEMICAL FUNCTIONALIZATION ACROSS THE LENGTH SCALES}

\author{
PROEFSCHRIFT
}

\author{
ter verkrijging van \\ de graad van doctor aan de Universiteit Twente, \\ op gezag van de rector magnificus, \\ prof. dr. H. Brinksma, \\ volgens besluit van het College voor Promoties \\ in het openbaar te verdedigen \\ op vrijdag 11 februari 2011 om 12:45 uur \\ door \\ Joost Duvigneau \\ geboren op 11 mei 1981 \\ te Winterswijk
}


Dit proefschrift is goedgekeurd door:

Promotor: prof. dr. G. J. Vancso

Assistent-promotor: prof. dr. H. Schönherr 
This Thesis is dedicated to my family 



\section{Table of Contents}

Chapter 1 General Introduction 1

1.1 Introduction 1

1.2 Concept of this Thesis 5

1.3 References 8

Chapter 2 Development of Scanning Thermal Lithography for Localized 11 Thermal Chemical Surface Functionalization

2.1 An introduction to atomic force microscopy 11

2.2 From characterization to manipulation: scanning probe lithography 14

2.3 Development of thermal scanning probes 20

2.4 Calibration of temperature 24

2.5 Heat transport from resistively heated AFM cantilevers 30

2.6 Scanning thermal lithography 34

2.7 Conclusions 38

$2.8 \quad$ References and notes 39

Chapter 3 Nanoscale Thermal AFM of Polymers: Transient Heat Flow 45 Effects

3.1 Introduction $\quad 46$

3.2 Results and discussion $\quad 49$

3.2.1 Heat induced localized PET crystallization $\quad 50$

3.2.2 PDMS film thickness dependent thermal expansion 54

3.2.3 Simplified steady state 1D model 57

$\begin{array}{lll}3.3 & \text { Conclusions } & 60\end{array}$

$\begin{array}{lll}3.4 & \text { Experimental } & 61\end{array}$

3.5 References and notes 63

Chapter 4 NanoTA of Thin Polymer Films: Tip-Sample Interface 65 Temperature Effects on the Onset of Indentation.

4.1 Introduction 66

4.2 Results and discussion $\quad 69$

4.2.1 NanoTA: substrate thermal conductivity effect 69 
4.2.2 nTMA of PS, PMMA and PC on silicon substrates 74

4.2.3 Estimation of $\Delta T_{i}$ for PS, PMMA and PC on silicon substrates 76

4.2.4 Monodisperse $\mathrm{PS}_{20}$ and $\mathrm{PS}_{650}$ films on silicon substrates 77

4.3 Conclusions 81

$\begin{array}{lll}4.4 & \text { Experimental } & 81\end{array}$

4.5 References and notes 83

Chapter 5 Surface Wetting Behavior of Poly(isoprene)-block-Poly(ferrocenyl- $\quad 87$ dimethylsilane) Crystals During Heating and Melting Unveiled by AFM and NanoTA

$\begin{array}{lll}5.1 & \text { Introduction } & 88\end{array}$

$\begin{array}{lll}5.2 & \text { Results and discussion } & 90\end{array}$

5.2.1 Differential scanning calorimetry of $\mathrm{PI}_{76}-b$ - $\mathrm{PFS}_{76}$

5.2.2 Deposition of $\mathrm{PI}_{76}-b-\mathrm{PFS}_{76}$ at $\mathrm{SiO}_{2}$ and $\mathrm{HOPG} 93$

5.2.3 Nanoscale thermal analysis of $\mathrm{PI}_{76}-b$-PFS ${ }_{76}$ crystals 94

5.2.4 Isothermal AFM of $\mathrm{PI}_{76}-b$ - $\mathrm{PFS}_{76}$ at elevated temperatures 95

$\begin{array}{lll}5.3 \text { Conclusions } & 100\end{array}$

5.4 Experimental 100

$\begin{array}{lll}5.5 & \text { References } & 102\end{array}$

Chapter 6 Reactive Imprint Lithography: Combined Topographical Patterning 105 and Chemical Surface Functionalization of Poly(styrene)-blockPoly(tert-butyl acrylate) Films

$\begin{array}{lll}6.1 & \text { Introduction } & 106\end{array}$

$\begin{array}{lll}6.2 & \text { Results and discussion } & 107\end{array}$

6.2.1 AFM investigation of the pattern transfer to $\mathrm{PS}_{2092}-b-\mathrm{P} t \mathrm{BA}_{1055}$ films $\quad 108$ during RIL

6.2.2 Transmission FTIR spectroscopy on $\mathrm{PS}_{2092}-b$-P $t \mathrm{BA}_{1055}$ films after RIL 110

6.2.3 Surface layer composition and functionality investigated with angle 111 resolved XPS

6.2.4 Fluorescence microscopic analyses of fluoresceinamine and 117 $\mathrm{MeO}-\mathrm{PEG}_{113}-\mathrm{NH}_{2}$ functionalized $\mathrm{PS}_{2092}-b-\mathrm{P} t \mathrm{BA}_{1055}$ films

6.3 Conclusions

$\begin{array}{lll}6.4 & \text { Experimental } & 120\end{array}$

$\begin{array}{lll}6.5 & \text { References and notes } & 122\end{array}$ 
Chapter 7 Scanning Thermal Lithography of PtBA Block Copolymer Films

\section{for Bioconjugation}

$\begin{array}{llr}7.1 & \text { Introduction } & 126\end{array}$

$\begin{array}{lll}7.2 & \text { Results and discussion } & 128\end{array}$

$\begin{array}{ll}\text { 7.2.1 Mechanism of thermolysis } & 128\end{array}$

$\begin{array}{lll}\text { 7.2.2 Thermolysis kinetics } & 131\end{array}$

7.2.3 Apparent activation energy of $t$ BA ester thermolysis 133

7.2.4 Chemical activation and surface immobilization of fluoresceinamine 134

$\begin{array}{lll}\text { 7.2.5 Local thermal surface functionalization } & 135\end{array}$

$\begin{array}{lll}7.3 & \text { Conclusions } & 139\end{array}$

$\begin{array}{lll}7.4 & \text { Experimental } & 139\end{array}$

$\begin{array}{lll}7.5 & \text { References and notes } & 142\end{array}$

Chapter 8 Tailored tert-Butyl Ester Protected Carboxylic Acid Functionalized 145 (Meth)acrylate Polymer Platforms for SThL

8.1 Introduction 146

$\begin{array}{lll}8.2 & \text { Results and discussion } & 148\end{array}$

$\begin{array}{lll}\text { 8.2.1 Film formation } & 148\end{array}$

8.2.2 Thermal decomposition reactions in $\mathrm{MA}_{20}$ and $\mathrm{A}_{20} \quad 149$

8.2.3 Derivatization of thermally deprotected $\mathrm{MA}_{20}$ and $\mathrm{A}_{20}$ bulk samples $\quad 154$

8.2.4 Improved thermal mechanical properties for SThL 155

8.2.5 SThL on $\mathrm{MA}_{20}$ samples 159

8.2.6 SThL on $\mathrm{A}_{20}$ films 161

$\begin{array}{lll}8.3 \text { Conclusions } & 164\end{array}$

$\begin{array}{lll}8.4 & \text { Experimental } & 165\end{array}$

$\begin{array}{lll}8.5 & \text { References and notes } & 168\end{array}$

$\begin{array}{ll}\text { Outlook } & 171\end{array}$

$\begin{array}{ll}\text { Summary } & 173\end{array}$

$\begin{array}{ll}\text { Samenvatting } & 177\end{array}$

Acknowledgements $\quad 181$

$\begin{array}{ll}\text { Curriculum Vitae } & 185\end{array}$ 



\section{Chapter 1 \\ General Introduction}

\subsection{Introduction}

Nature provides us with numerous examples of the most intriguing and complex processes occurring at interfaces at the macromolecular length scale. Hence it is a continuous source of inspiration for materials scientists and biologists. Thus a prime objective in materials science is to control and mimic these well defined and efficient processes at artificial interfaces. For instance, Spatz and coworkers ${ }^{1}$ have reported on the considerable effect of separation distance, ranging from 23 to $85 \mathrm{~nm}$, of single integrins on cell adhesion. Single integrins were conjugated to hexagonally packed gold dots $(<8 \mathrm{~nm})$ on glass surfaces. It was shown that $73 \mathrm{~nm}$ integrin separation distances and above resulted in limited cell adhesion and spreading. In a recent study, Spatz and coworkers ${ }^{2}$ expanded their earlier efforts to study the effect of lateral integrin pattern order on cell adhesion. Disordered integrin patterns with averaged separation distances over $70 \mathrm{~nm}$ showed increased cell adhesion compared to the less adhesive ordered integrin patterns. To be able to study and eventually exploit such phenomena in e.g. (bio)sensors and early diagnostics, there is an increasing need to develop and fabricate biologically reactive interfaces with well defined topographical and/or chemical domains across the length scales.

In order to meet the growing demands in ability to address, manipulate and fabricate a broad range of materials and devices in the $100 \mathrm{~nm}$ down to the sub $10 \mathrm{~nm}$ length scale, currently available as well as newly to be developed nanofabrication approaches are obliged to evolve into cost effective, robust and widely applicable techniques with high throughputs. ${ }^{3}$ This need stimulated us to enhance our knowledge and explore novel routes for the fabrication of spatially controlled chemically well defined nanostructured surfaces.

The conventional nanolithography approaches, i.e. optical and directed beam, are well established techniques mostly applied for semiconductor fabrication, despite some limitations in meeting these requirements. Sub $100 \mathrm{~nm}$ spatial resolutions can be obtained with photolithography, ${ }^{4}$ however the necessary technically challenging resolution enhancement approaches often result in a cost efficiency penalty. Despite the mentioned challenges as well as resist resolution limitations often encountered, the semiconductor micro fabrication industry has reached the $32 \mathrm{~nm}$ node $^{5}$ and is currently pursuing the $22 \mathrm{~nm}$ node. Direct writing with charged particles ${ }^{6}$ (i.e. electrons or ions) offers great flexibility in pattern design at sub $50 \mathrm{~nm}$ spatial resolution, however the serial nature of the process (resulting in low throughputs) 
combined with the expensive equipment used results in a poor cost efficiency. In addition, the conventional approaches are often not applicable to the wide variety of organic and biological materials used and they often comprise of multi-step processes. $^{7}$ More recent established nanolithography approaches as alternatives to the conventional approaches are nanoimprint lithography (NIL), ${ }^{8}$ soft lithography ${ }^{9}$ and scanning probe lithography (SPL). ${ }^{10}$ Where NIL mainly focuses on the development of nanometer sized topographical patterns by imprinting polymer films at elevated temperatures with specially designed molds, soft lithography focuses on the fabrication of patterns with distinguished chemical functional patterns in the sub micrometer range to sub-100 nanometer. These techniques have developed and continue to develop into cost effective, robust, widely applicable nanotechnology tools for the controlled topographical and chemical nanoscale patterning of a wide diversity of substrates.

On the other hand, scanning probe microscopy (SPM, i.e. atomic force microscopy and scanning tunneling microscopy) ${ }^{11}$ based approaches, have attracted considerable attention, mainly related to their capability to achieve sub-10 nanometer down to the atomic ${ }^{12}$ length scale imaging and patterning resolution on a wide variety of substrates (among which biomolecules and semiconductor devices) without being restricted to high vacuum environments and conductive substrates. Furthermore these are relatively low cost and easy to use approaches. Therefore it is also not surprising that the introduction of scanning probe lithography (SPL) occurred shortly after the invention of SPM. ${ }^{13}$ In SPL the typical nanometer sized sharp tip apex is used to locally apply a stimulus to a specific region on a surface to modify its surface properties in terms of topography and/or (chemical) functionality. The applied stimuli were mainly mechanical, electrical or chemical in nature. ${ }^{14} \mathrm{~A}$ few well known examples of SPL are NanoShaving, ${ }^{15}$ localized surface oxidation ${ }^{16}$ and DIP-Pen nanolithography. ${ }^{17}$

SPLs based on single probes have a limitation in pattering throughput, which possibly prevents their economic viable utilization in large scale applications. This was overcome by the development of massively parallel operated arrays of probes for thermal probe based data storage devices ${ }^{18}$ and DIP-Pen nanolithography. ${ }^{19}$ State of the art cantilever arrays are reported to have over 5000 and 55000 cantilevers integrated in one chip to be operated simultaneously and independently for the IBM Millipede ${ }^{18}$ and DIP-Pen nanolithography, ${ }^{19}$ respectively.

With the introduction of batch fabricated heatable AFM probes by $\mathrm{Mamin}^{20}$ in 1996 for thermal mechanical data storage applications, ${ }^{21}$ they became available for exploring other scanning thermal microscopy approaches (SThM). Initially the utilization of these probes remained limited to massive parallel thermal mechanical 
data bit writing/reading and nanoscale thermal analysis. The use of these probes as localized heat sources for thermal chemical surface modifications, referred to as scanning thermal lithography (SThL), was reported on more recently (after the start of this project) by King and coworkers and by our group (Figure 1.1). ${ }^{22}$

\section{Scanning Thermal Lithography}

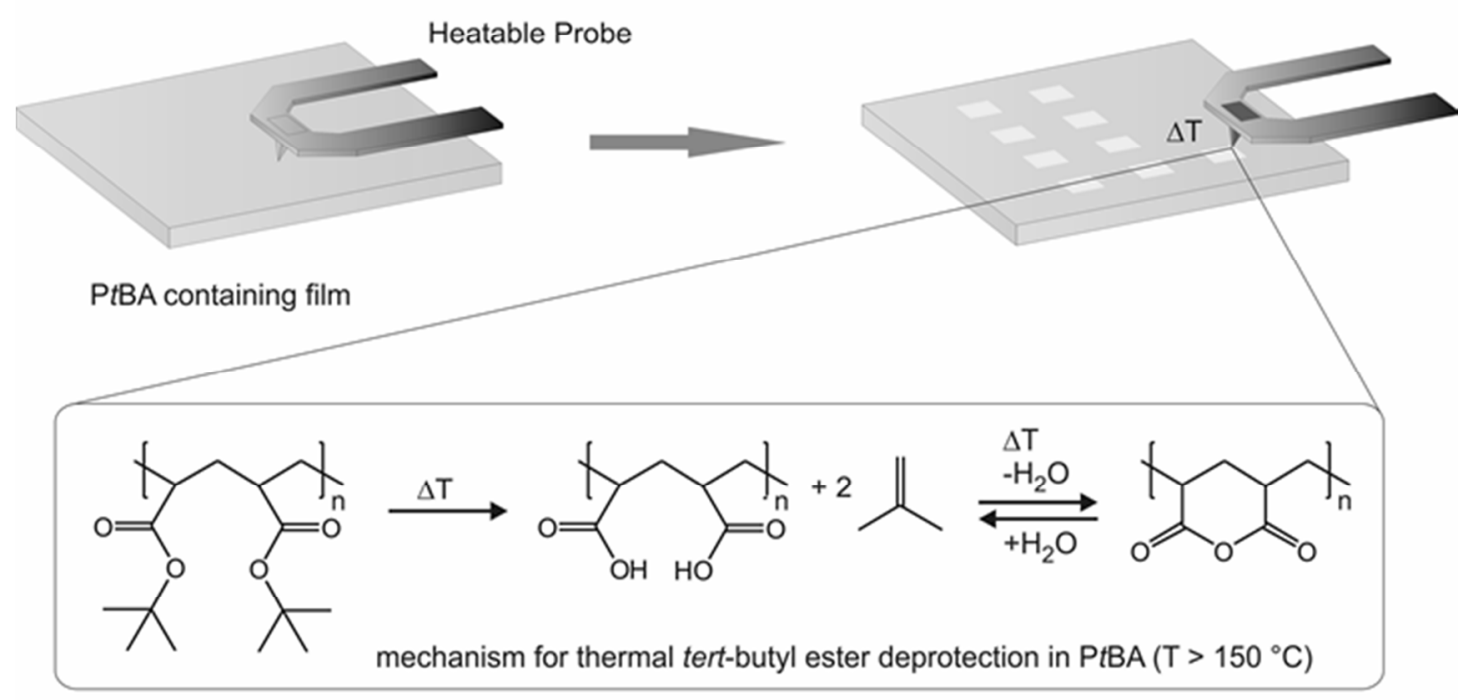

Figure 1.1: Schematic of scanning thermal lithography. The heatable AFM probes with resistive Joule heaters embedded at the cantilever end are utilized for the highly localized spatially controlled chemical surface modifications. For example surface exposed tert-butyl esters in poly(tert-butyl acrylate) containing polymer films are known to be thermally cleavable to yield the corresponding carboxylic acid and anhydride moieties after the subsequent loss of isobutylene and water, respectively. ${ }^{22 b}$

Thermally induced reactions, especially surface reactions on polymers represent a class of yet largely unexplored reactions in terms of controlled surface modification and structuring. Nealy and coworkers ${ }^{23}$ induced a cylindrical to spherical morphology transition in poly(styrene)-block-poly(tert-butyl acrylate) thin films by thermal cleavage of the tert-butyl ester. Böker et $a .^{24}$ reported on the thermal functionalization of poly(styrene)-block-poly(isoprene[graft-perfluoroacyl]) block copolymer surfaces via thermal cleavage of the perfluorinated side chains at $340{ }^{\circ} \mathrm{C}$. Only Böker et al. ${ }^{24}$ mentioned the possible surface derivatization of exposed chemical functional groups after thermolysis. Heat transport at small length scales is less restricted compared to known limitations for conventional lithography (given by the diffraction limit) or various drawbacks of other scanning probe lithographies (SPLs). Hence, SThL is expected to provide an exiting, alternative avenue for spatially controlled, highly localized surface modification and/or manipulation (Figure 1.1). 
In addition to a localized heat source for SThL, i.e. the heatable AFM probes, a suitable platform with efficient surface chemistry is also required. Polymer (thin) films are already widely applied and still being further explored for numerous applications in for example semiconductor devices, ${ }^{25}$ bio reactive platforms ${ }^{26}$ and (bio)sensors ${ }^{27}$ due to their robustness in all kinds of processing steps, reproducibility and ease of preparation as well as their quasi 3D nature for loading with e.g. biomolecules. The block copolymer film platform based on poly(styrene)-blockpoly(tert-butyl acrylate) (PS- $b$-P $t \mathrm{BA})$, recently introduced by us, meets the requirements for the fabrication of tailored (bio)reactive interfaces. ${ }^{28}$ Thin films of this block copolymer show symmetric wetting, for example, on oxide covered surfaces, where the PtBA block is in contact with both the substrate and the air at the two interfaces. Due to microphase separation of the block copolymer, the internal structure of the films comprises continuous PS cylinders which provide the already mentioned robustness in a broad range of processing conditions. The reactive PtBA skin layer exposed at the film surface on the other hand can be exploited to obtain very robust functionalized architectures. This process comprises the acid-catalyzed deprotection of the tert-butyl ester groups and subsequent wet chemical grafting reactions. Via utilization of new concepts in soft lithography, patterns down to the $300 \mathrm{~nm}$ size range were fabricated on these polymer platforms. ${ }^{29}$ Besides their instability in the presence of acid, the $t \mathrm{BA}$ ester groups are known to undergo thermal deprotection reactions, ${ }^{30}$ yielding surface exposed carboxylic acid and anhydride groups. Both are versatile functionalities for further conjugation with various biologically interesting molecules. Therefore it is expected that $\mathrm{P} t \mathrm{BA}$ based polymer films can be exploited into versatile platforms that can be $i$ ) locally thermochemically functionalized and ii) subsequently wet chemically grafted with e.g. biologically interesting molecules, down to the macromolecular level.

Like previously mentioned, thermally activated surface reactions were not described in detail before. Only very recently Knoll and coworkers ${ }^{31}$ have reported on the fabrication of 3D structures in polymer films via SThL induced local polymer decomposition with approximately $40 \mathrm{~nm}$ resolution. In addition, King and coworkers $^{32}$ have demonstrated the applicability of SThL to polymer films with surface exposed tetrahydropyran carbamate protected amine groups. After thermal decompostion of the tertrahydropyran carbamate bonds the amide functionalities were wet chemically modified to various other chemical functionalities. These examples show the feasibility of SThL as an attractive approach for spatially controlled thermally induced surface modifications.

Extended insights in $i$ ) the stability of polymer films at elevated temperatures during SThL with respect to thermomechanical surface deformations and chemical reaction mechanisms at the relatively short reaction times applied and in $i$ ) the heat 
transport close to the heated probe-tip contact interface for various film thicknesses, were obtained by us. These results may prove to be useful for the further development of SThL as an attractive alternative approach for the fabrication of nanostructured polymer film platforms with distinguished topographical and/or chemical information.

\subsection{Concept of this Thesis}

The central aim of the research described in this Thesis is the investigation of SThL as a new SPM based approach for sub $50 \mathrm{~nm}$ thermal chemical surface functionalization to provide polymer platforms for localized targeted (bio)conjugation in subsequent processing steps. Thermal chemical surface functionalization of tert-butyl (meth)acrylate based polymer systems into carboxylic acid functional domains across the length scales is presented. Heat transport arising from heated cantilevers in contact with polymer substrates is discussed in detail. The effect of copolymer film composition on the resolution of TSPL is systematically presented, starting from PS- $b$-P $t$ BA block copolymer films and subsequently extended to more tailored tert-butyl ester exposed reactive interfaces.

In Chapter 2 a literature overview on the general topics presented in this Thesis is provided. Following a brief introduction to AFM and SPL, a more detailed overview on the development of SThL is given in a historical perspective. Highlighted are cantilever design, temperature calibration and an overview of SThM applications.

In Chapter 3 the heat transport from a heated cantilever in contact with polymer films is described. The thermal probe induced surface crystallization of amorphous poly(ethylene terephthalate) (PET) reveals a short range high temperature gradient close to the tip-polymer contact interface. In addition, film thickness dependent poly(dimethylsiloxane) (PDMS) thermal expansion measurements provide insight in the long range thermal transport during typical non steady state NanoTA conditions. The results established a detailed insight in the thermal transport routes and revealed the importance of taking heat transport from the heated cantilever through the air gap between the heated cantilever and polymer film into account for optimizing the resolution of SThM based approaches.

The range of thermal transport is further elucidated in Chapter 4, which describes deviations in effective tip-sample interface temperatures $\left(T_{i}\right)$ from the calibrated $T_{i}$ for NanoTA measurements on supported polymer thin films. With decreasing film thickness an increase in measured softening temperature was observed for films on good thermally conductive solid supports (e.g. silicon or glass). This effect was more pronounced for silicon compared to glass, which is ascribed to the approximately 100 times higher thermal conductivity of silicon compared to glass. The close proximity of the good thermally conductive solid support resulted in effective 
lowering of $T_{i}$. The measured deviations of $T_{i}$ from the calibrated $T_{i}$ were several tens of degrees Celsius for $50 \mathrm{~nm}$ thick poly(styrene) (PS), polycarbonate (PC) and poly(methyl methacrylate) (PMMA) films on silicon substrates. For thicknesses lower than approximately $350 \mathrm{~nm}$ for PC and approximately $600 \mathrm{~nm}$ for PMMA as well as PS films on silicon substrates, significant deviations of $T_{i}$ from the calibrated $T_{i}$ are to be expected.

In Chapter 5 the melting behavior of poly(isoprene)-block-poly(ferrocenyldimethylsilane) (PI- $b$-PFS) block copolymer crystals on hydrophilic and hydrophobic surfaces is presented. Having established the melting trajectory of the PFS crystalline core, the in situ isothermal temperature controlled AFM study is expanded to the introduction of heatable AFM probes for rapid nanoscale thermal analysis (NanoTA) of the macromolecular PI- $b$-PFS crystalline architectures. NanoTA on single, $15 \mathrm{~nm}$ thick PI- $b$-PFS crystals, deposited on silicon was found to be below the physical limit of the technique. This was ascribed to the low sensitivity of the heated cantilevers for the thin crystals as well as to the decreased probe tip contact interface in proximity of the silicon substrate. The latter effect was expected from the results described in Chapter 3 and Chapter 4.

In Chapter 6 reactive imprint lithography (RIL) is introduced as a new one-step approach for the combined thermal chemical surface functionalization and topographical patterning of PS- $b$-P $t$ BA block copolymer films. Imprinting above the tert-butyl ester deprotection temperature yields topographically patterned poly(styrene)- $b$-poly(acrylic acid) (PS- $b$-PAA) films with surface exposed carboxylic acid and anhydride groups. The availability of these groups for wet chemical grafting reactions was confirmed by the EDC/NHS mediated coupling of amino containing, biologically relevant molecules. Besides showing that RIL is an interesting complementary approach for the preparation of (bio)reactive surfaces in a one step process, it also demonstrates the feasibility of thermal chemical surface functionalization of tert-butyl ester protected acrylates and as such this chapter forms one of the pillars for an in depth investigation of exploiting SThL on tert-butyl ester containing polymers.

Having established the basic needs and understanding concerning thermal transport from heated AFM cantilevers and thermal chemical surface functionalization, Chapter 7 continues with describing the development of SThL. The kinetics and thermal deprotection mechanism of PS- $b$-P $t$ BA are studied in detail followed by the localized thermal deprotection of these films with SThL. The smallest obtained domain size remained above the critical sub 100 nanometer length scale, which is a result of the poor thermomechanical properties of the block copolymer films at the relatively high temperatures used for tert-butyl ester deprotection 
$\left(\sim 250^{\circ} \mathrm{C}\right)$, leading to a combination of thermochemical surface functionalization and thermomechanical surface deformation.

Therefore in Chapter $\boldsymbol{8}$ the development of tailored tert-butyl ester protected carboxylic acid group containing (meth)acrylate based copolymer substrates for SThL is described. Crosslinking of the polymer films yields thermomechanically stable polymer films for the development of thermochemical SThL without observing thermomechanical surface deformations. The difference in thermal deprotection mechanism for the methacrylate and acrylate based films (depolymerization versus ester deprotection, respectively) is presented in terms of apparent activation energy for the corresponding processes. The observed higher apparent activation energy $\left(E_{a}\right)$ for tert-butyl ester deprotection in the tert-butyl acrylate system compared to the lower $E_{a}$ for poly(tert-butyl methacrylate) depolymerization, significantly enhances the resolution of thermochemical surface functionalization via SThL. The chemical functionality of the deprotected PAA domains after SThL was confirmed with fluorescence microscopy after wet chemical grafting of a fluorescent dye. The highest achievable resolution with the available heatable AFM cantilevers is approximately $20 \mathrm{~nm}$ which is below the tip radius of curvature of the used probes.

The research described in this Thesis contributed significantly to the development of SThL. Finally in the Outlook, directions for future research are provided. For instance, polymer film platforms that contain multiple protected chemical functionalities that can be thermochemically deprotected with SThL are envisaged as one of the improvements that have to be achieved in future research. These developments will contribute to $i$ ) the enhancement of the chemical functionalities that can be simultaneously introduced to the exposed reactive surface domains as well as to $i$ ) an increase in platform preparation throughput. 


\subsection{References}

1 Anold, M.; Cavalcanti-Adam, E. A.; Glass, R.; Blummel, J.; Eck, W.; Kantlehner, M.; Kessler, H.; Spatz, J. P. Chem. Phys. Chem. 2004, 5, 383.

2 Huang, J. H.; Grater, S. V.; Corbellinl, F.; Rinck, S.; Bock, E.; Kemkemer, R.; Kessler, H.; Ding, J. D.; Spatz, J. P. Nano Lett. 2009, 9, 1111.

3 a) Bang, J.; Jeong, U.; Ryu, D. Y.; Russell, T. P.; Hawker, C. J. Adv. Mater. 2009, 21, 4769. b) Gates, B. D.; Xu, Q. B.; Stewart, M.; Ryan, D.; Willson, C. G.; Whitesides, G. M. Chem. Rev. $\mathbf{2 0 0 5}, 105,1171$.

4 Buxboim, A.; Bar-Dagan, M.; Frydman, V.; Zbaida, D.; Morpurgo, M.; Bar-Ziv, R. Small 2007, 3, 500 .

5 a) Wu, B. Q.; Kumar, A. J. Vac. Sci. Technol. B 2007, 25, 1743. b) Kozawa, T.; Tagawa, S. J. Photopolym. Sci. Technol. 2009, 22, 51.

6 a) Christman, K. L.; Schopf, E.; Broyer, R. M.; Li, R. C.; Chen, Y.; Maynard, H. D. J. Am. Chem. Soc. 2009, 131, 521. b) Tseng, A. A. Small 2005, 1, 924.

7 a) Xie, X. N.; Chung, H. J.; Sow, C. H.; Wee, A. T. S. Mat. Sci. Eng. R. 2006, 54, 1. b) García, R.; Martinez, R. V.; Martinez, J. Chem. Soc. Rev. 2006, 35, 29.

8 a) Guo, L. J. J. Phys. D Appl. Phys. 2004, 37, R123. b) Chou, S. Y.; Krauss, P. R.; Renstrom, P. J. Appl. Phys. Lett. 1995, 67, 3114. c) Chou, S. Y.; Krauss, P. R.; Renstrom, P. J. Science 1996, 272, 85. d) Truskett, V. N.; Watts, M. P. C. Trends Biotechnol. 2006, 24, 312.

9 a) Xia, Y. N.; Whitesides, G. M. Angew. Chem. Int. Ed. 1998, 37, 551. b) Weibel, D. B.; DiLuzio, W. R.; Whitesides, G. M. Nat. Rev. Microbiol. 2007, 5, 209.

10 a) Tseng, A. A.; Notargiacomo, A.; Chen, T. P. J. Vac. Sci. Technol. B 2005, $23,877$.

11 a) Wouters, D.; Schubert, U. S. Angew. Chem. Int. Ed. 2004, 43, 2480. b) Krämer, S.; Fuierer, R. R.; Gorman, C. B. Chem. Rev. 2003, 103, 4367.

12 a) Eigler, D. M.; Schweizer, E. K. Nature 1990, 344, 524. b) Becker, R. S.; Golovchenko, J. A.; Swartzentruber, B. S. Nature 1987, 325, 419. c) Heller, E. J.; Crommie, M. F.; Lutz, C. P.; Eigler, D. M. Nature 1994, 369, 464.

13 a) Ringger, M.; Hidber, H. R.; Schlögl, R.; Oelhafen, P.; Güntherodt, H. J. Appl. Phys. Lett. 1985, 46, 832. b) Nagahara, L. A.; Oden, P. I.; Majumdar, A.; Carrejo, J. P.; Graham, J.; Alexander, J. Proc. SPIE Int. Soc. Opt. Eng. 1992, 1639, 171.

14 Nyffenegger, R. M.; Penner, R. M. Chem. Rev. 1997, 97, 1195.

15 a) Xu, S.; Liu, G. Y. Langmuir 1997, 13, 127. b) Liu, G. Y.; Xu, S.; Qian, Y. L. Accounts. Chem. Res. 2000, 33, 457.

16 Dagata, J. A.; Schneir, J.; Harary, H. H.; Evans, C. J.; Postek, M. T.; Bennett, J. Appl. Phys. Lett. 1990, 56, 2001.

17 a) Piner, R. D.; Zhu, J.; Xu, F.; Hong, S. H.; Mirkin, C. A. Science 1999, 283, 661. b) Ginger, D. S.; Zhang, H.; Mirkin, C. A. Angew. Chem. Int. Ed.2004, 43, 30. c) Jaschke, M.; Butt, H. J. Langmuir 1995, 11, 1061.

18 Binnig, G. K.; Cherubini, G.; Despont, M.; Dürig, U. T.; Eleftheriou, E.; Pozidis H.; Vettiger, P. In: B. Bhushan, Editor, Handbook of Nanotechnology, $2^{\text {nd }}$ ed., Springer Science and Business Media Inc., Heidelberg, 2006, p. 1457-1486.

19 a) Salaita, K.; Wang, Y. H.; Fragala, J.; Vega, R. A.; Liu, C.; Mirkin, C. A. Angew. Chem. Int. Ed. 2006, 45, 7220. b) Lenhert, S.; Sun, P.; Wang, Y. H.; Fuchs, H.; Mirkin, C. A. Small 2007, 3, 71 .

20 Mamin, H. J. Appl. Phys. Lett. 1996, 69, 433. 
21 Vettiger, P.; Despont, M.; Drechsler, U.; Dürig, U.; Häberle, W.; Lutwyche, M. I.; Rothuizen, H. E.; Stutz, R.; Widmer, R.; Binnig, G. K. IBM J. Res. Dev. 2000, 44, 323.

22 a) Szoszkiewicz, R.; Okada, T.; Jones, S. C.; Li, T. D.; King, W. P.; Marder, S. R.; Riedo, E. Nano Lett. 2007, 7, 1064. b) Duvigneau, J.; Schönherr, H.; Vancso, G. J. Langmuir 2008, 24, 10825.

23 a) La, Y. H.; Edwards, E. W.; Park, S. M.; Nealey, P. F. Nano Lett. 2005, 5, 1379. b) La, Y. H.; Stoykovich, M. P.; Park, S. M.; Nealey, P. F. Chem. Mater. 2007, 19, 4538.

24 a) Böker, A.; Reihs, K.; Wang, J. G.; Stadler, R.; Ober, C. K. Macromolecules 2000, 33, 1310. b) Böker, A.; Herweg, T.; Reihs, K. Macromolecules 2002, 35, 4929.

25 a) Murphy, A. R.; Fréchet, J. M. J. Chem. Rev. 2007, 107, 1066. b) Thompson, B. C.; Fréchet, J. M. J. Angew. Chem. Int. Ed. 2008, 47, 58.

26 a) Mizutani, A.; Kikuchi, A.; Yamato, M.; Kanazawa, H.; Okano, T. Biomaterials 2008, 29, 2073. b) Lussi, J. W.; Michel, R.; Reviakine, I.; Falconnet, D.; Goessl, A.; Csucs, G.; Hubbell, J. A.; Textor, M. Prog. Surf. Sci. 2004, 76, 55. c) Chen, C. S.; Jiang, X. Y.; Whitesides, G. M. MRS Bull. 2005, 30, 194.

27 a) MacBeath, G.; Schreiber, S. L. Science 2000, 289, 1760. b) Fernandes, T. G.; Diogo, M. M.; Clark, D. S.; Dordick, J. S.; Cabral, J. M. S. Trends Biotechnol. 2009, $27,342$.

28 a) Feng, C. L.; Embrechts, A.; Bredebusch, I.; Bouma, A.; Schnekenburger, J.; García-Parajó, M.; Domschke, W.; Vancso, G. J.; Schönherr, H. Eur. Polym. J. 2007, 43, 2177. b) Feng, C. L.; Embrechts, A.; Vancso, G. J.; Schönherr, H. Eur. Polym. J. 2006, 42, 1954. c) Feng, C. L.; Vancso, G. J.; Schönherr, H. Langmuir 2005, 21, 2356. d) Feng, C. L.; Vancso, G. J.; Schönherr, H. Langmuir 2007, 23, 1131.

29 a) Feng, C. L.; Embrechts, A.; Bredebusch, I.; Schnekenburger, J.; Domschke, W.; Vancso, G. J.; Schönherr, H. Adv. Mater. 2007, 19, 286. b) Embrechts, A.; Feng, C. L.; Mills, C. A.; Lee, M.; Bredebusch, I.; Schnekenburger, J.; Domschke, W.; Vancso, G. J.; Schönherr, H. Langmuir 2008, 24, 8841 .

30 a) Litmanovich, A. D.; Cherkezyan, V. O. Eur. Polym. J. 1984, 20, 1041. b) Duvigneau, J.; Cornelissen, S.; Bardají Valls, N.; Schönherr, H.; Vancso, G. J. Adv. Funct. Mater. 2010, 20, 460.

31 a) Pires, D.; Hedrick, J. L.; De Silva, A.; Frommer, J.; Gotsmann, B.; Wolf, H.; Despont, M.; Dürig, U.; Knoll, A. W. Science 2010, 328, 732. b) Knoll, A. W.; Pires, D.; Coulembier, O.; Dubois, P.; Hedrick, J. L.; Frommer, J.; Dürig, U. Adv. Mater. 2010, 22, 3361.

32 Wang, D. B.; Koda V. K.; Underwood, W. D.; Jarvholm, J. E.; Okada, T.; Jones, S. C.; Rumi, M.; Dai, Z. T.; King, W. P.; Marder, S. R.; Curtis, J. E.; Riedo, E. Adv. Funct. Mater. 2009, 19, 3696. 



\section{Chapter 2}

\section{Development of Scanning Thermal Lithography for Localized Thermal Chemical Surface Functionalization}

This chapter will provide an overview of the most important developments concerning scanning probe lithography (SPL) and scanning thermal microscopy (SThM). First a basic introduction to atomic force microscopy (AFM) is given by discussing its two most common used modes of operation, often referred to as contact mode $(\mathrm{CM})$ and tapping mode (TM) AFM, respectively. In addition two more advanced AFM modes of operation are discussed. Furthermore a brief overview of different SPL approaches will be provided. The focus of this chapter will be a detailed review of the development of SThM, including cantilever design, temperature calibration and the most prominent examples of scanning thermal lithography (SThL).

\subsection{An introduction to atomic force microscopy}

Since the introduction of scanning probe microscopy (SPM) with the invention of scanning tunneling microscopy (STM) ${ }^{1}$ in 1982, this class of surface characterization tools have been rapidly growing to mature platforms that find wide spread usage all over the world. Major contributions to this success are ascribed to the development of AFM in 1986 by Binnig and coworkers. ${ }^{2}$ In STM a bias voltage is applied between a sharp conductive tip and the target sample. When the tip is in close proximity of the target sample (within the atomic range), electrons begin to tunnel through the gap from the tip to the sample or vice versa depending on the sign of the bias applied. The exponential dependence of the resulting tunneling current as a function of the tip sample distance provides STM with sub ångström vertical and sub nanometer lateral resolutions. ${ }^{3,4}$ The main limitation of STM is the need for conductive surfaces/ substrates. Since AFM relies on the measurement of attractive and/or repulsive forces acting between a sharp tip in close proximity of a surface, the need for conductive surfaces in SPM vanished. Hence, it became possible to examine polymers and biologically relevant samples. Since its inception AFM was frequently adapted to different approaches being capable to measure surface material properties such as mechanical, thermal, optical or magnetic properties with possible nanometer resolution with a wide variety of probes in a broad range of environments. Among these approaches are force spectroscopy, ${ }^{5,6}$ chemical force spectroscopy, ${ }^{7,8}$ scanning thermal microscopy, ${ }^{9,10}$ Harmonix ${ }^{11,12}$ and magnetic force microscopy. ${ }^{13,14}$ It is not desirable to provide a comprehensive overview here, hence the reader is directed to several review papers ${ }^{15-20}$ and books. ${ }^{21-23}$ Here, the two most common modes 
(i.e. contact mode and tapping mode AFM) of operation will be briefly introduced followed by a short introduction to two more advanced modes of AFM, i.e. Harmonix and single molecule force spectroscopy.

In the most basic mode of AFM operation, very often referred to as contact mode AFM, a sharp probe tip (with typical tip radius of curvature of 10-100 nm) positioned at the end of a flexible cantilever is brought into contact with a surface of interest. As a result of attractive and/or repulsive forces acting between the probe and surface, the cantilever vertically deflects towards or away from the surface, respectively. ${ }^{24}$ This deflection is monitored with an optical sensor system, in which a low noise laser or a super bright LED is aligned on the back of the cantilever and its reflection is projected via a mirror on a position sensitive photo diode (Figure 2.1). In constant height mode (Figure 2.1B) the changes in deflection $(\Delta \mathrm{D})$ are measured while raster scanning either the sample or the cantilever to obtain a topographic image of the sample surface. In constant force mode (Figure 2.1B), a feedback loop maintains a preset vertical deflection (i.e. a constant force) of the cantilever while scanning the surface to obtain a topographic image. The maintained deflection is directly proportional to the normal load applied by the probe to the surface. ${ }^{25}$ Besides measuring the deflection and height signals, one also can measure lateral forces acting on the probe when scanning a sample perpendicular to the cantilever main axis. The resulting torsional bending of the cantilever is proportional to the friction force of the probe-sample contact (Figure 2.1A). ${ }^{26}$

In tapping mode AFM (Figure 2.1C) ${ }^{27}$ the feedback loop keeps an oscillating cantilever (typically at a resonance frequency of several tens of $\mathrm{kHz}$ up to $350 \mathrm{kHz}$ ) at constant amplitude rather than maintaining a constant deflection. One advantage of tapping mode AFM compared to contact mode AFM is that due to the oscillation of the cantilever during raster scanning a surface in tapping mode AFM, friction forces between tip and sample are significantly reduced. ${ }^{28}$ Especially for the examination of 'soft' surfaces, like polymers or biologically relevant samples (e.g. cells), one can significantly reduce sample damage when operating the AFM in tapping mode. ${ }^{27,29}$ Despite the reduced lateral interaction forces the probe tip reaches close to or makes contact with the sample surface during each oscillation. Hence, it experiences attractive and repulsive forces, e.g. adhesion, viscoelasticity, charge, etc. These forces result in a phase shift with respect to the oscillating amplitude. The phase shift is very sensitive to variations in material properties and is therefore an excellent signal to visualize the presence of variations in surface composition of heterogeneous sample surfaces. 
A Atomic Force Microscopy
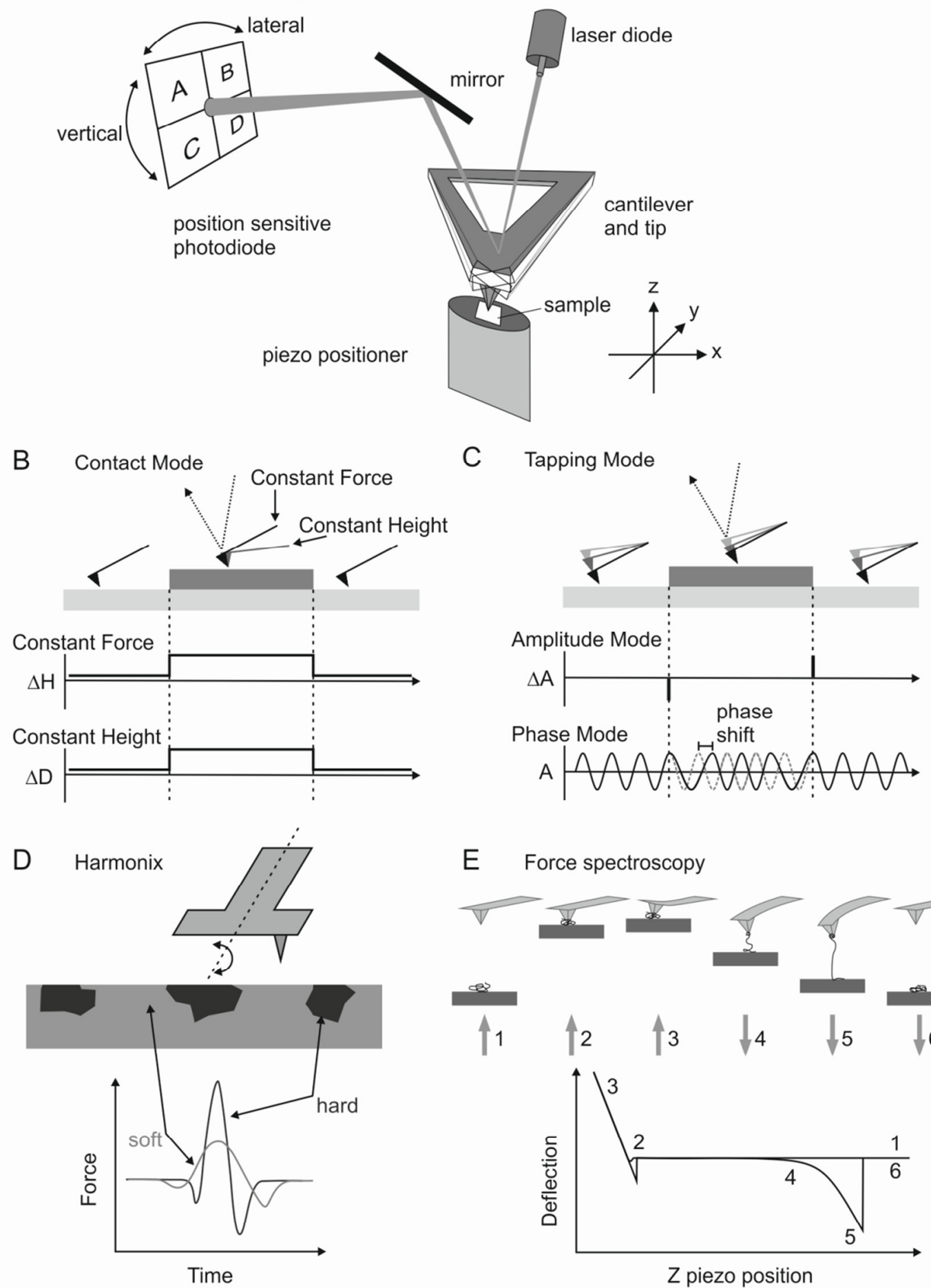

E Force spectroscopy

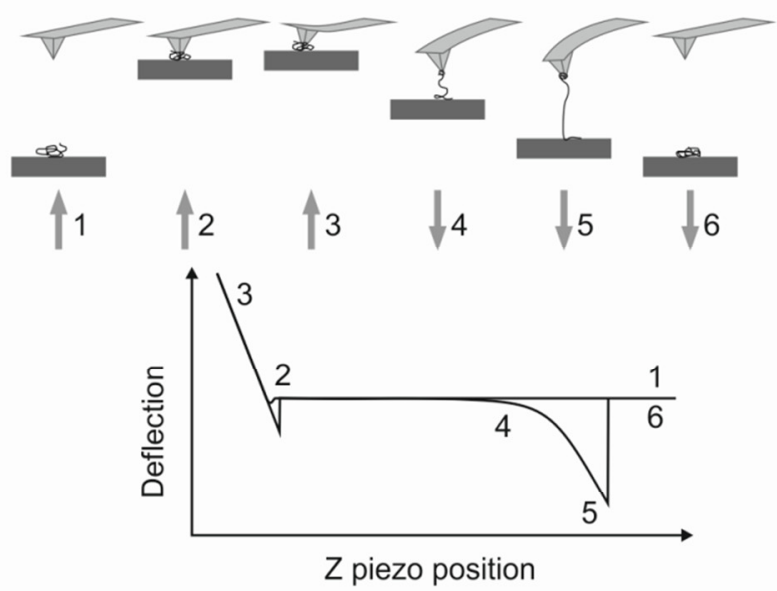

Figure 2.1: Schematic representation of atomic force microscopy with a sample scanner (A). The two most well known modes of operation, contact mode and tapping mode are shown in $(B)$ and $(C)$, respectively. In addition, schematics of Harmonix (D) and force spectroscopy (E) are shown. Images $A$ and $E$ where reproduced with permission from references 30 and 31 , respectively. 
The recently introduced Harmonix AFM mode ${ }^{11,12}$ (Figure 2.1D) enables the realtime simultaneous topographic and mechanical property mapping of materials (e.g. polymers) with a sub $10 \mathrm{~nm}$ spatial resolution. This technique relies on the use of specially designed cantilevers with laterally offset tips operating in conventional tapping mode AFM. Tip-sample interactions cause the cantilever to twist along the longitudinal axis, generating torsional vibrations (i.e. higher harmonics in the $\mathrm{MHz}$ regime). These torsional vibrations are captured with high speed data capture electronics from which force curves are extracted at microsecond temporal resolution. From the captured force curves the mechanical surface parameters, i.e. stiffness, adhesion, modulus and dissipation, can be quantified. Compared to, for example, force-volume and nanoindentation measurements, which can provide quantitative surface mechanical properties of polymers, Harmonix has a much higher temporal and lateral resolution. Its disadvantage is that the quantification of the reduced elastic modulus is restricted to the use of the Derjaguin-Muller-Toporov (DMT) model. ${ }^{12}$

Single molecule force spectroscopy provides another example of the high spatial resolution possible to achieve with AFM. In principle mechanical properties of a single macromolecule can be determined by stretching a molecule that is attached with one end to a substrate and the other end to the AFM probe tip while recording a force distance curve (Figure 2.1E). The magnitude of forces that can be measured with available AFM cantilevers ranges from femtonewtons ( $\mathrm{fN}$ ) to a few nanonewtons $(\mathrm{nN})$. The advantages of this technique are that the measurements are performed in well controlled environments (e.g. temperature, salt concentration, solvent, etc.) without being restricted to the use of aqueous environments.

\subsection{From characterization to manipulation: scanning probe lithography}

In addition to the relative ease with which SPM was adapted as a versatile tool for examining surfaces with nanometer lateral resolution, the proximity of the probe tip close to a sample surface can easily be exploited to turn the SPM into a nanoscale lithography tool. In fact, circa 3 years after the introduction of STM the first example of STM based lithography was shown. ${ }^{32}$ Ringger et al. prepared a line pattern (line width $\sim 16 \mathrm{~nm}$ ) via scanning with a STM tip over an atomically flat $\mathrm{Pd}_{81} \mathrm{Si}_{19}$ surface. The mechanism for line formation, although at that time not fully understood, was ascribed to either polycondensation of a thin hydrocarbon layer present at the surface or to reduction of the silicon oxide layer. ${ }^{32}$ Later Eigler et al. ${ }^{33}$ demonstrated the selective positioning of deposited xenon atoms on a nickel $<110>$ surface to form the letters IBM (Figure 2.2). In addition, Dagata et al. ${ }^{34}$ have shown the local oxidation of a hydrogen passivated silicon surface with a STM operated in air. 

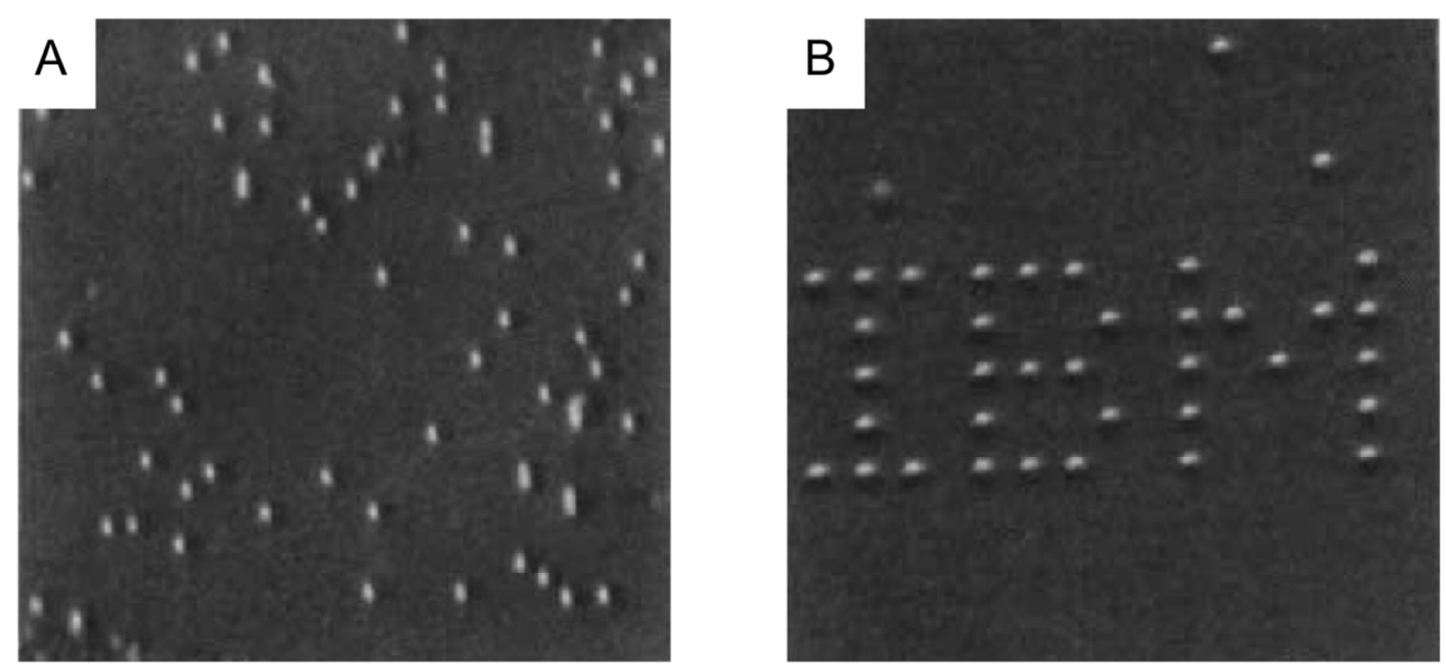

Figure 2.2: STM images before $(A)$ and after (B) selective positioning of single xenon atoms deposited on a nickel $<110\rangle$ surface. Reprinted by permission from Macmillan Publishers Ltd: reference 33, copyright 1990.

These examples demonstrated the high resolution that can be achieved with STM lithography. Drawbacks of this approach remain the often required controlled environments (e.g. UHV), its limitation to conductive surfaces and its generally low patterning speed.

Complementary to STM lithography, AFM based lithography has provided a fascinating diversity of approaches for sub $100 \mathrm{~nm}$ lithography, which is mainly related to its outstanding capability to operate at sub $10 \mathrm{~nm}$ (imaging) resolution while not being limited by material selection or restrictions in the surrounding medium. Also the relative ease with which an atomic force microscope can be transferred into a surface modification tool has made it an attractive approach for sub 100 nanometer lithography. In principle six main categories of stimuli applied by an AFM probe in contact or proximity of a sample surface can be distinguished, known to be: i) mechanical, ii) electrical, iii) thermal, iv) optical, v) molecular transport and $v i$ ) chemical. These have been extensively reviewed by others. ${ }^{35-45}$ In the following a more generalized impression based on the mentioned tip-sample interaction categories will be provided, including some of the most notable examples for AFM lithography. SPL based on thermal stimuli will be reviewed in Section 2.5.

One of the earliest examples of lithography where mechanical forces were applied with an atomic force microscope (scanning force lithography (SFL)) was scratching grooves into a polycarbonate (PC) film with a relatively stiff AFM cantilever. ${ }^{46}$ This so called static ploughing approach resulted in the formation of $\sim 70 \mathrm{~nm}$ width and $\sim 10 \mathrm{~nm}$ deep grooves into the PC film. Kunze et al. ${ }^{47,48}$ showed 
that for scan directions deviating from the cantilevers longitudinal axis during static ploughing, the cantilever might bend as a result of the torsion forces acting on the tip. Hence pattern irregularities are possibly introduced during SFL. They introduced dynamic ploughing, which is best described by hammering with the tip in TM AFM onto the surface, in order to induce topographical changes to the surface. They were able to write $\sim 40 \mathrm{~nm}$ width letters in a thin resist layer (Figure 2.3).

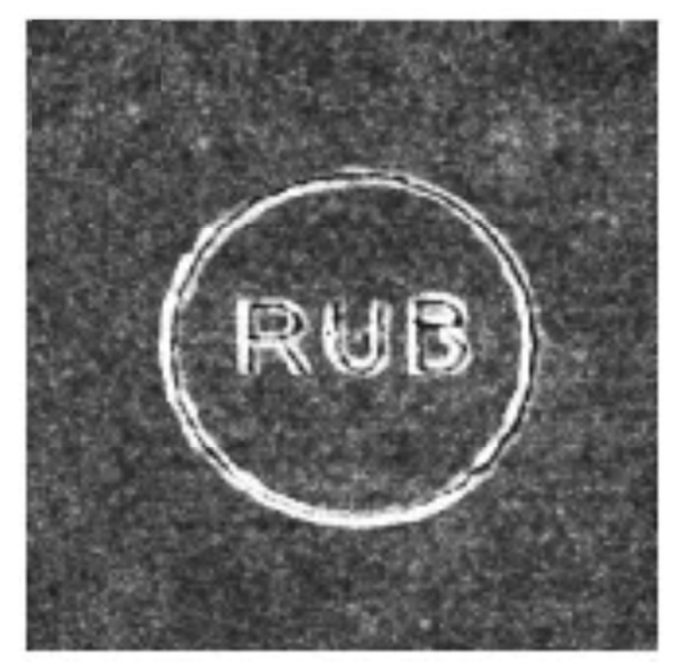

Figure 2.3: AFM image of a patterned area prepared by dynamic ploughing in a polymeric resist. Patterning speed was $5 \mu \mathrm{m} \mathrm{s}^{-1}$. The image area represents $2.8 \mu \mathrm{m} \times 2.8 \mu \mathrm{m}$. Reprinted with permission from reference 47. Copyright 1999, American Institute of Physics.

In addition to ploughing, nanoshaving was introduced for the selective removal of self-assembled monolayers (SAMs). ${ }^{42}$ Liu et al. ${ }^{49}$ reported on the selective removal of $\mathrm{C}_{10} \mathrm{SH}$ (1-decanethiol) molecules absorbed on gold surfaces by nanoshaving performed in 2-butanol. The exposed gold surface was backfilled during scanning with $\mathrm{C}_{18} \mathrm{SH}$ (1-octadecanethiol, ODT) molecules from solution. The typical loading force applied by the tip for the removal of $\mathrm{C}_{10} \mathrm{~S}$ molecules was around $5.2 \mathrm{nN}$.

In contrast to the selective removal of molecules with SFL, the AFM probe tip was also exploited for the delivery of molecules on surfaces. Jaschke and Butt ${ }^{50}$ reported in 1995 for the first time on the deposition of organic material from an AFM probe tip to a substrate. They noticed that ODT 'contamination' deposited on a probe tip, that was used one day before to image a gold substrate in millimolar ODT solution in ethanol, was transferred uncontrolled to the freshly prepared mica surfaces. Later on (1999) Mirkin and coworkers ${ }^{51}$ improved this method and named it Dip-Pen nanolithography (NL), which is probably the most well known example for this SPL approach. In Dip-Pen NL an "ink" coated AFM probe tip is used for the selective 
deposition of molecules (e.g. thiols or proteins) on a surface. Originally they reported on the deposition of ODT molecules (i.e. the ink) on a gold surface (Figure 2.4). ${ }^{52}$ Later on this method was used for example for the fabrication of protein arrays in a multi-step process. ${ }^{53}$ During Dip-Pen NL the ink molecules have to be transported from the tip to the substrate via molecular diffusion through the water meniscus between the tip and sample surface. Hence, control over the water meniscus is important for achieving optimized pattering resolutions $(<50 \mathrm{~nm})$ with good patterning reproducibility. Deposition of different types of ink molecules on one substrate requires multiple tips coated with the corresponding inks. Read out of the prepared area requires another probe or different environmental conditions, to reduce the risk of surface contamination with ink molecules from the used coated tip during Dip-Pen NL. In addition to the selective deposition from a coated probe tip other designs have also been introduced. For instance Elwenspoek and coworkers ${ }^{54,55}$ have reported on the fabrication of a micromachined fountain pen within a dual cantilever design. They incorporated an ink reservoir within the cantilever base to enable a continuous fluid supply to one cantilever. The second cantilever could be used for the characterization of the prepared patterns. The continuous liquid supply allowed controlled patterning compared to the less controlled deposition reported on by Jaschke and Butt. ${ }^{50}$ Simple drying of the reservoir stops the ink flow and as a result no more material is deposited on the surface.

A

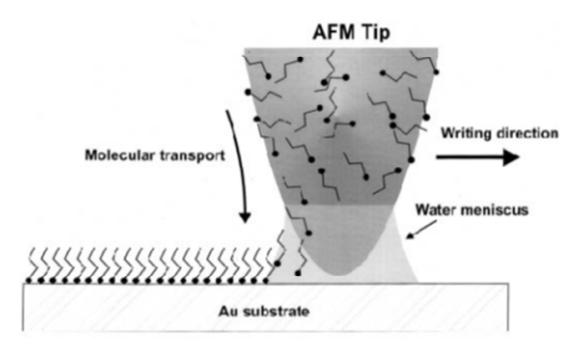

B

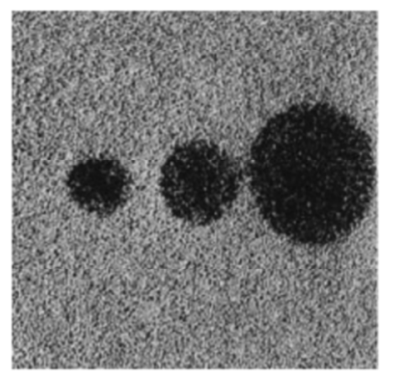

C

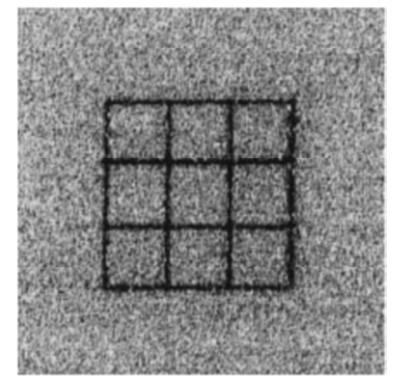

Figure 2.4: (A) schematic of Dip-Pen NL. (B) and (C) AFM lateral force images of $4 \mu \mathrm{m} \times 4 \mu \mathrm{m}$ gold areas after selective deposition of ODT via Dip-Pen NL. From reference 52. Reprinted with permission from AAAS.

In addition to remove or deposit molecules, the AFM probe tip can be applied to deliver a stimulus that locally modifies the surface chemical composition and/or topography. Majumdar and coworkers ${ }^{56}$ have demonstrated the use of a gold coated tip as a localized electron source for the spatially controlled exposure of an ultrathin poly(methyl methacrylate) (PMMA) resist layer to electrons. They reported on the formation of a line pattern with line widths of $35 \mathrm{~nm}$ and a periodicity of $68 \mathrm{~nm}$. 
Furthermore, Xie et al. ${ }^{57}$ reported on the formation of PMMA pillars as a result of ionic dissociation in the water meniscus in the near proximity of a negatively biased gold coated $\mathrm{Si}_{3} \mathrm{~N}_{4}$ AFM tip (Figure 2.5). In addition, photo chemical oxidation of SAMs of mercaptoundecanoic acid on gold via scanning near-field optical microscopy (SNOM) lithography $(\lambda=244 \mathrm{~nm})^{58}$, followed by the selective wet chemical etching of the unmasked substrate was reported to produce $\sim 55 \mathrm{~nm}$ wide trenches in gold surfaces. ${ }^{59}$

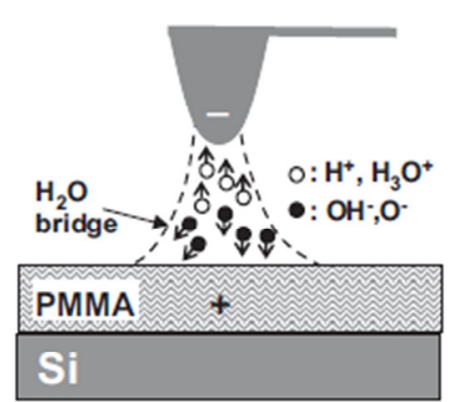

(a)

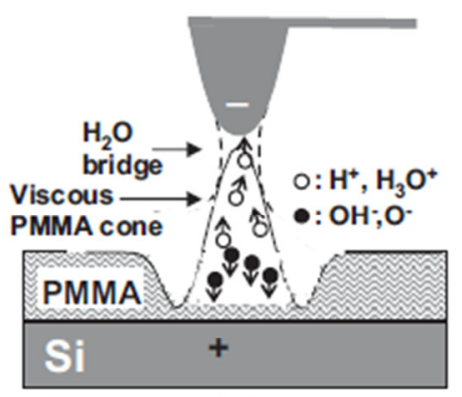

(b)

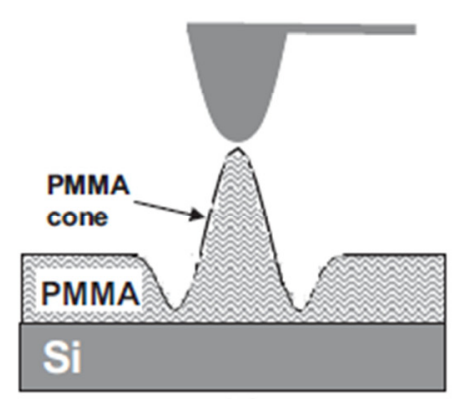

(c)

Figure 2.5: Schematic showing the formation of PMMA conical pillars on a $80 \mathrm{~nm}$ thick PMMA film. It was believed that resistive Joule heating underneath the tip in addition to the ionic dissociation of water molecules in the meniscus upon biasing the gold coated AFM tip (A), is followed by the water assisted ionic conduction of the viscous polymer (B). After release of the bias a stable PMMA pillar is formed (C). Adapted from reference 57.

Furthermore the AFM tip can be functionalized with catalytically active molecules in order to catalyze a chemical reaction in proximity of the tip. Reinhoudt and coworkers ${ }^{60}$ reported on the locally acid catalyzed hydrolysis of surface exposed silyl ether moieties in a bis(ortho-tert-butyldimethyl-siloxyundecyl)disulfide (TBDMS) SAMs on gold surfaces by scanning the surface with a 2-mercapto5-benzimidazole sulfonic acid $\left(\mathrm{MBS}-\mathrm{H}^{+}\right)$functionalized gold-coated AFM tip (Figure 2.6).

The above discussed examples have all contributed to the embodiment of AFM lithography as a workhorse for cutting edge NL performed by researchers all over the world. However most of these approaches suffer from limitations or combinations thereof in sample choice, probe fabrication, probe tip wear, patterning speed and the need for multiple tip processes or the sometimes required more complex instrumentation. 


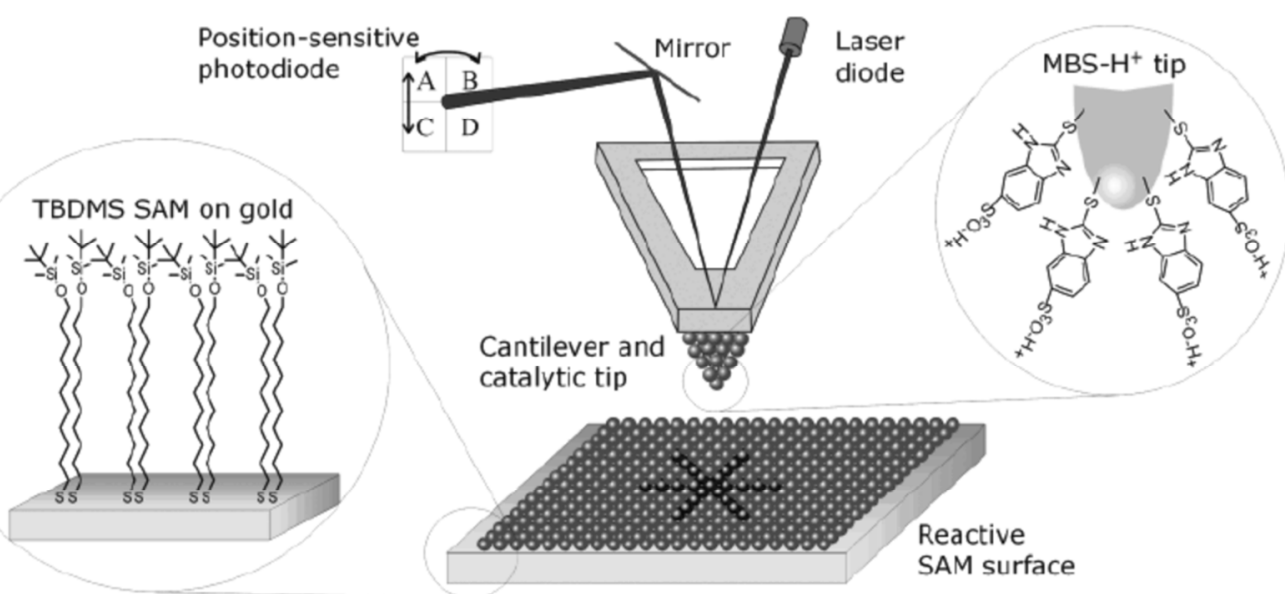

Figure 2.6: Schematic of scanning catalytic probe lithography performed on TBDMS SAMs on gold surfaces using a MBS- $H^{+}$functionalized gold coated AFM tip. Reprinted with permission from reference 60. Copyright 2004 American Chemical Society.

These issues have to be properly addressed before these SPL approaches can be applied on a broad scale in economically viable processes. The most important issue, i.e. the low patterning throughput, has been addressed by Esashi and coworkers, ${ }^{61}$ Vettiger and coworkers ${ }^{62}$ and Mirkin and coworkers ${ }^{63,64}$ who developed arrays of 108, 4096 and 55000 cantilevers operating in parallel for nanoscratching of a photo resist layer, thermomechanical data writing/reading (i.e. IBMs' Millipede project, Figure 2.7) or in Dip-Pen NL, respectively.

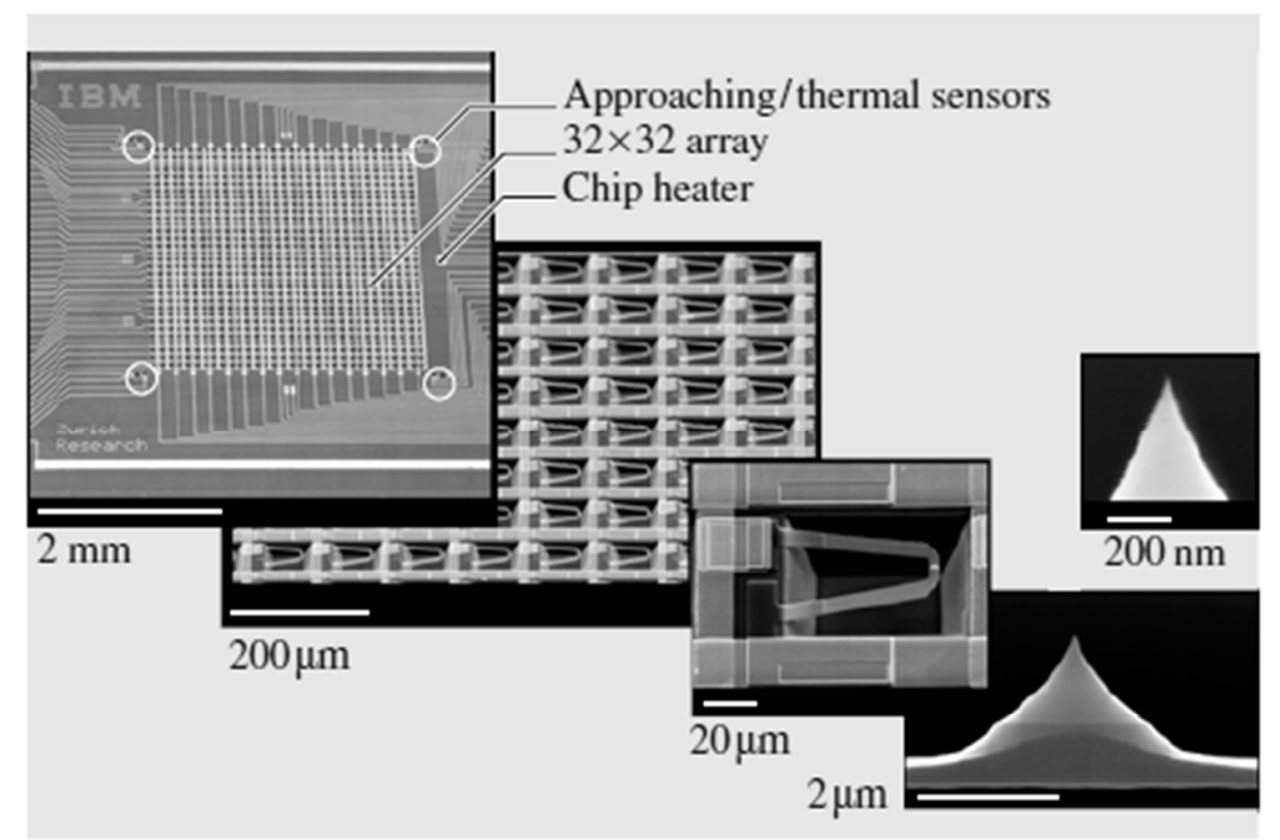

Figure 2.7: SEM images of a $32 \times 32$ heatable cantilever array fabricated by IBM, part of that array and one individual cantilever. Furthermore SEM images of the sharpened tips are shown. Republished with kind permission from Springer Science+Business Media: Handbook of Nanotechnology. ${ }^{62}$ 
The IBM Millipede team reported that their heatable AFM probes were capable of operating at data bit write rates of $\sim 12.5 \mathrm{kBps}$ (for one probe) at very high data storage densities $\left(\sim 1\right.$ Tbit $\left.^{\text {inch }}{ }^{-2}\right){ }^{65,66}$ For modern data storage devices, this writing speed is relatively low when compared to a commercially available solid state drive having a data write rate of 140 MBps. ${ }^{67}$ However compared to other AFM based lithographies the numbers for thermal mechanical data bit writing rates are considered to be high. After the start of this research project the first examples of SThL were reported on by King et al. ${ }^{68}$ and by us. ${ }^{69}$ The former reported a theoretical maximum achievable writing speed of $\sim 30 \mathrm{~mm} \mathrm{~s}^{-1}$ for SThL. This number was based on the estimated tip sample contact radius $(30 \mathrm{~nm})$ and the thermal time constant of the heated cantilever when in contact with a substrate $(\sim 1 \mu \mathrm{s}) .{ }^{68}$ For example when compared to Dip-Pen NL where typical dot writing times require probe dwell times of $80 \mathrm{~ms}^{70}$ and lines can be written at $\sim 1 \mu \mathrm{m} \mathrm{s}^{-1}$ or less, ${ }^{71}$ SPL based on thermal AFM probes is fast. Another advantage of using heated probes is that the same probe can be used to write and read out the prepared patterns, without the risk of surface contamination. Furthermore it was recognized that applying heat to a polymer surface with an AFM probe tip is not limited to thermomechanical deformation but can also be used for thermochemical patterning. Therefore these probes were considered to be the ideal candidates for the development of an alternative approach for nanoscale chemical surface functionalization of polymer films. The development of heatable AFM probes will be discussed in the next section, followed by heated cantilever temperature calibration. Finally an overview of their use in SThL is provided.

\subsection{Development of thermal scanning probes}

From a historical point of view several approaches have been used to achieve controlled AFM probe tip heating. These will be discussed below. The most important designs are shown in Figure 2.8 and Figure 2.9. In 1992 Mamin and Rugar ${ }^{72}$ reported for the first time on the pulsed laser heating of a gold coated AFM tip to produce indents with a width of $100 \mathrm{~nm}$ in a PMMA film. Heated probe tip temperatures above $120^{\circ} \mathrm{C}$ were achieved with $300 \mathrm{~ns}$ laser pulses (power several $\mathrm{mW}$ ). Laser heating of AFM tips was later on mainly reported for the spatially controlled nanopatterning of surfaces ${ }^{73,74}$ as well as for the development of heated tip temperature characterization methods (See Section 2.4). ${ }^{75,76}$ The required second external laser for cantilever tip heating as well as the alignment of this laser on the probe tip, resulted in an impractical approach for their implementation in large scale applications. Hence, it was recognized that the use of probes with internal microscale heaters would greatly simplify the operating procedure. 
A

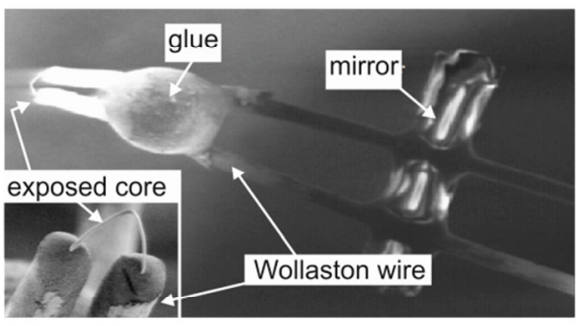

C

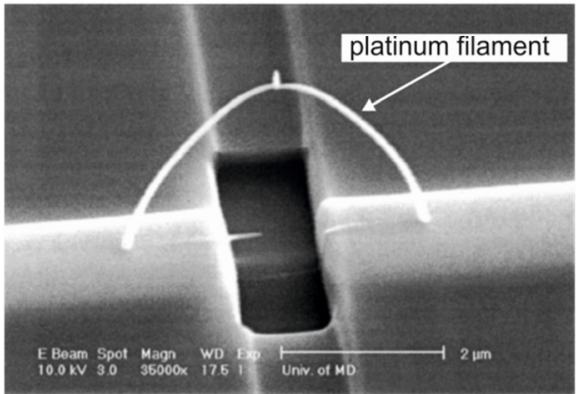

$\mathrm{B}$

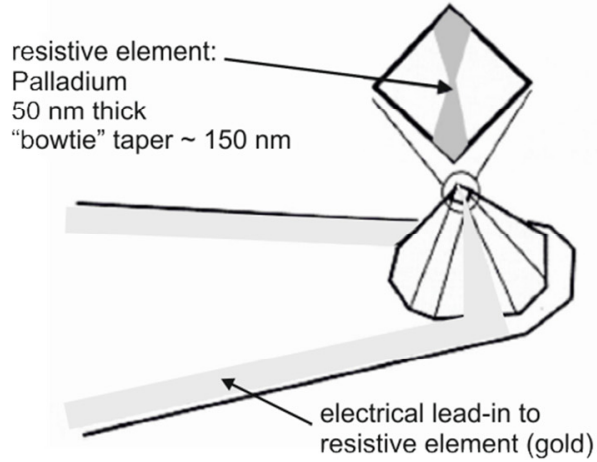

$\mathrm{D}$

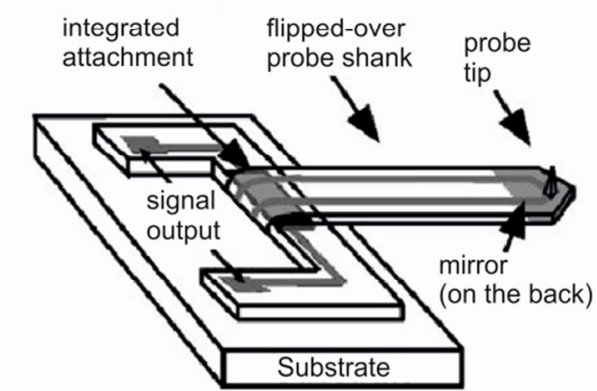

Figure 2.8: SEM images ( $A$ and $C$ ) or schematics $(B$ and $D)$ of the different fabricated thermal probe designs utilized for thermal sensing or localized heating. Wollaston wire probes $(A),{ }^{79}$ tapered palladium resistor probes $(B),{ }^{84}$ platinum filament probes $(C),{ }^{86}$ and flexible polyimide probes $(D){ }^{90}$ These images were adapted from the respectively noted references, with kind permission from the corresponding publishers.

In 1994, West and coworkers ${ }^{77,78}$ reported on the development of the first probes with an integrated resistive heater. These were made from a Wollaston process wire, consisting of a $5 \mu \mathrm{m}$ thick platinum core surrounded by a $75 \mu \mathrm{m}$ silver corona. After bending the wire into a sharp apex, the silver was etched away, exposing approximately $200 \mu \mathrm{m}$ of the platinum core which forms the tip. Upon applying a voltage over the wire, the surface exposed platinum apex forms a high electrical resistance area, which results in localized Joule heating of the wire at the apex (Figure 2.8A). These wires can be operated as a thermal sensor ${ }^{79,80}$ or as a resistive heater. $^{81,82}$ Their main limitations were the poor lateral resolution, typically over $1 \mu \mathrm{m}^{83}$ compared to the AFM imaging resolution $(<50 \mathrm{~nm})$ and their serial manual assembly process. These issues were addressed by Pollock and coworkers. ${ }^{84}$ They reported on the development of batch fabricated heatable AFM probes. They combined wafer scale micromachining with multiple level electron beam lithography to produce a tapered palladium wire on top of a flattened AFM tip (Figure 2.8B). Taper widths of $150 \mathrm{~nm}$ were achieved and the electrical resistance was on the order of $250 \Omega$ up to $1000 \Omega$. Rangelow and coworkers ${ }^{85,86}$ have reported on the development of platinum filament probe tips on an AFM cantilever with a patterned 
development of platinum filament probe tips on an AFM cantilever with a patterned aluminum layer (that served as the electrical contacts). Positioning of a platinum tip at the apex of the platinum filament resulted in a typical probe tip radius of curvature of $\sim 20 \mathrm{~nm}$ (Figure 2.8C). Remaining disadvantages of these probes were their relatively high spring constant, poor electrical shielding and limited thermal isolation, which made them unsuitable for use in biological and aqueous environments. ${ }^{87}$ These issues were tackled by the batch fabricated flexible polyimide cantilevers with an incorporated nickel/Wollaston resistor reported on by Gianchandani and coworkers (Figure 2.8D). ${ }^{88-90}$ The polyimide used reduced the thermal conduction to the environment and its low stiffness enabled the examination of softer samples. Their fabrication process, although batch, was still considered to be a complicated process in which the cantilever at a certain point has to be flipped over.

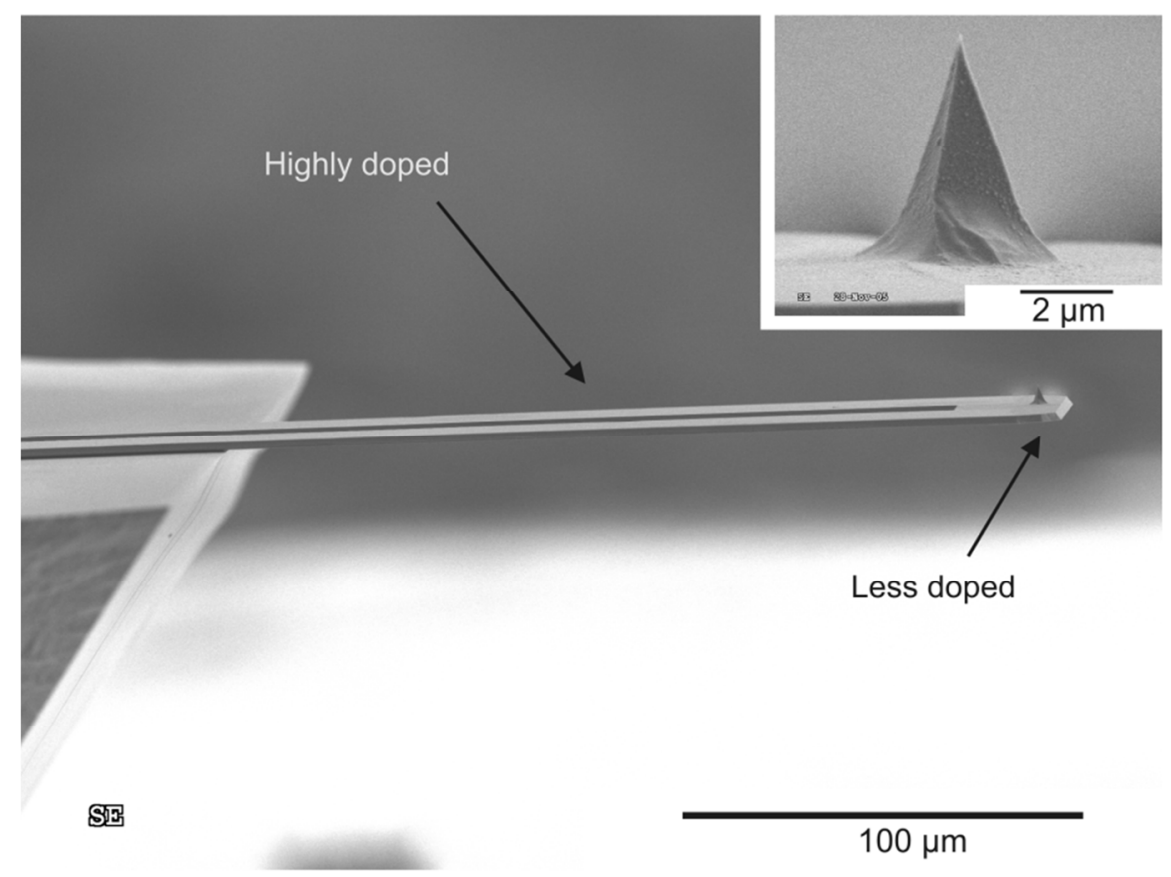

Figure 2.9: SEM image of a batch fabricated silicon AFM cantilever with an integrated resistive Joule heater embedded at the cantilever end, showing the highly phosphorous doped cantilever legs and the significantly lower doped cantilever end with the tip. The inset shows a SEM image of the tip. The lower doped region at the cantilever end enables highly localized resistive Joule heating upon applying a voltage over the cantilever legs. The tip height is approximately $4 \mu \mathrm{m}$ with a typical tip radius of curvature of $30 \mathrm{~nm}$. "SEM images courtesy of Kevin Kjoller, Anasys Instruments Corp."

Nowadays batch fabricated silicon AFM cantilevers with integrated Joule heaters embedded at the cantilever end are commercially available (Figure 2.9). ${ }^{91,92}$ They are mainly based on a design introduced by Mamin in $1996 .{ }^{93}$ Boron doped piezoresistive AFM cantilevers were integrated in an electrical circuit for the controlled AFM 
cantilever heating. The cantilever resistance was dominated by the cantilever legs $(\sim 2 \mathrm{k} \Omega$ ), which enabled localized heating with time constants of 300-500 $\mu \mathrm{s}$. In the same year Rugar and coworkers ${ }^{94}$ reported on the development of a micromachined single crystal silicon cantilever with an integrated resistive heater element embedded at the cantilever end. Due to the isolated and much smaller heater area size, heating and cooling time constants were reduced to approximately $30 \mu \mathrm{s}$. This fabrication route based on SOI processes was later adapted by King and coworkers. ${ }^{95-97}$ The latter reported on two-legged cantilevers consisting of highly boron doped legs $\left(1 \times 10^{20} \mathrm{~cm}^{-3}\right)$ with a lower dopant concentration at the bridge between the legs $\left(1 \times 10^{17} \mathrm{~cm}^{-3}\right)$. Hence the cantilever legs are electrically conductive whereas the bridge at the cantilever ends serves as a resistive Joule heater element with an approximate area of $8 \mu \mathrm{m} \times 16 \mu \mathrm{m}$. The electrical resistance of the reported cantilever was on the order of $1.7 \mathrm{k} \Omega$ which resulted in a maximum heater temperature of $\sim 560{ }^{\circ} \mathrm{C}$ for an input power of approximately $3 \mathrm{~mW}$. The probe tip, formed through an oxidation sharpening process ${ }^{98}$ had a tip radius of curvature of $\sim 20 \mathrm{~nm}$. These numbers are representative numbers for this type of batch fabricated cantilevers, but are known to vary for different cantilever designs. ${ }^{99,100}$

Table 2.1: List of specifications for two commercially available heatable AN2-type AFM probes. The numbers behind AN2- represent the length [ $\mu \mathrm{m}]$ of the cantilevers. ${ }^{91}$

\begin{tabular}{l|c|c}
\hline probe model & AN2-200 & AN2-300 \\
\hline \hline cantilever material & $\mathrm{Si}$ & $\mathrm{Si}$ \\
resistor material & doped Si & doped Si \\
length $[\mu \mathrm{m}]$ & $\sim 200$ & $\sim 300$ \\
thickness $[\mu \mathrm{m}]$ & $\sim 2$ & $\sim 2$ \\
tip height $[\mu \mathrm{m}]$ & $3-6$ & $3-6$ \\
spring constant $\left[{\left.\mathrm{N} \mathrm{m}^{-1}\right]}^{-1}\right.$ & $0.5-3$ & $0.1-0.5$ \\
resonant frequency $[\mathrm{kHz}]$ & $55-80$ & $15-30$ \\
tip radius of curvature $[\mathrm{nm}]$ & $<30$ & $<30$ \\
maximum controllable $\mathrm{T}\left[{ }^{\circ} \mathrm{C}\right]$ & 350 & 400 \\
contact mode imaging & Yes & Yes \\
tapping mode imaging & Yes & No
\end{tabular}

Due to the relatively simple micro fabrication processes used for the production of these cantilevers, their design was later on adopted and modified by others. ${ }^{101-104}$ In addition, based on their robustness, good performance and commercial availability, these probes were considered as the best candidates for the exploration of SThL as a new nanotechnology tool for controlled highly localized chemical surface 
functionalization (See Section 2.5). The commercially available probes are referred to as AN2-type and their main characteristics as provided by their manufacturer are shown in Table 2.1.

\subsection{Calibration of temperature}

For the development of SThL, knowledge of the heated probe temperature is required. Since the probe temperatures cannot be obtained directly a calibration step is necessary. While optical calibration methods are preferred since they are 'non-invasive' and often are able to spatially map an area of interest, they are generally not routine techniques. Techniques such as micro-infrared thermometry ${ }^{105}$ provide temperature resolutions below $1{ }^{\circ} \mathrm{C}$ with a spatial resolution of $\sim 5 \mu \mathrm{m}$ when using far-field diffraction limited optics. ${ }^{106,107}$ At this spatial resolution detailed information of the temperature distribution in a heated cantilever might be lost. Laser thermoreflectance ${ }^{108}$ has been reported to possess submicron resolution at gigahertz frequency monitoring of surface temperature changes. However with this method only relative temperature changes can be measured. ${ }^{109}$ For heated AFM cantilevers in principle four main approaches were reported by Nelson and King, ${ }^{110}$ including: i) single point calibration, ii) isothermal calibration, iii) Raman thermometry and iv) polymer melting point standards calibration. Based on their report these methods will be discussed here. Important to note is that the first three methods calibrated the heater area temperature $\left(T_{H}\right)$ whereas the polymer melting point standards method calibrates the temperature at the probe tip-sample interface $\left(T_{i}\right)$.

The easiest way of temperature calibration would be to use the single point method. This method starts with a theoretical prediction or measurement of the intrinsic temperature $\left(T_{\text {int }}\right)$, which is defined as the temperature at which the resistance of the cantilever has a maximum as a function of temperature (Figure 2.10). Initially the resistance of a heated silicon cantilever increases for increasing temperature. This is ascribed to the increased electron-phonon scattering at higher temperatures reducing the carrier mobility in silicon. For temperatures higher than $T_{i n t}$, excitation of electrons into the conduction band of the silicon starts to dominate the electrical resistance, which as a result starts to decrease with increasing temperature. This thermal runaway behavior for silicon is well understood and described elsewhere. ${ }^{111}$ Measurement of the cantilevers resistance at $T_{i n t}$ and correlation of this resistance to the predicted $T_{\text {int }}$ represents the single point measured for the calibration curve. Next a linear relationship between the cantilever resistance at room temperature and the resistance at $T_{i n t}$ is assumed for the construction of the cantilever temperature calibration curve for $T_{H}$. 


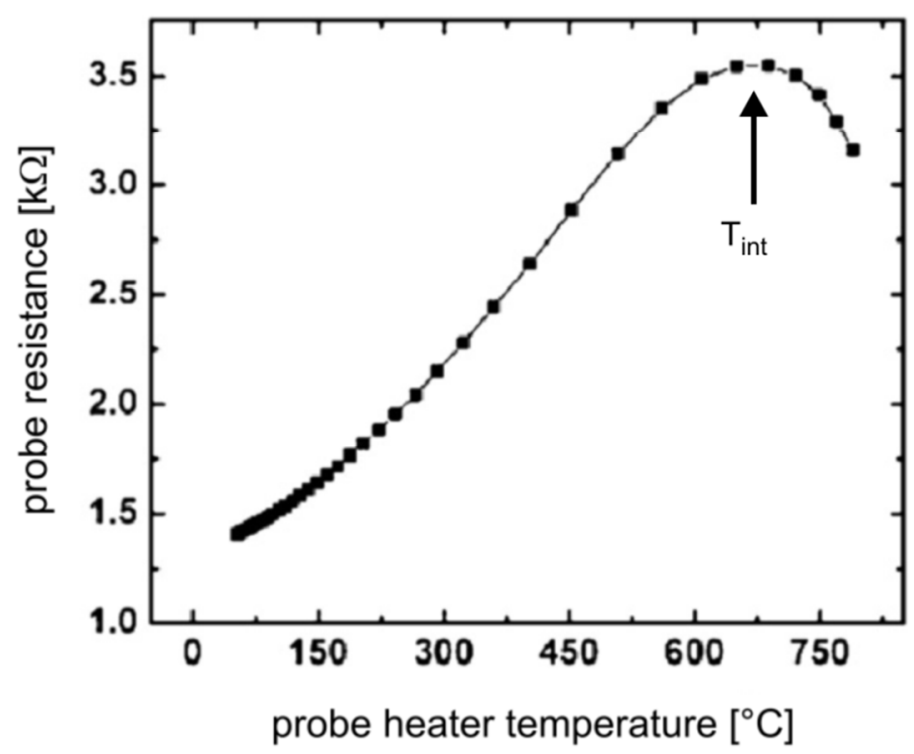

Figure 2.10: Typical cantilever resistance versus probe heater temperature curve for a heated silicon AFM cantilever. The black arrow points to $T_{\text {int }}$. Reprinted from reference 110 , with permission from Elsevier.

King and coworkers ${ }^{110}$ measured $T_{\text {int }}$ for a set of 25 cantilevers obtained from the same wafer with Raman thermometry (see below) to be between $500{ }^{\circ} \mathrm{C}$ and $1300{ }^{\circ} \mathrm{C}$, with a mean value of $810{ }^{\circ} \mathrm{C} \pm 160{ }^{\circ} \mathrm{C}$. The high variation in measured $T_{\text {int }}$ was ascribed to dopant concentration variations as a result of thickness deviations for the different cantilevers. Hence, a theoretical prediction of $T_{\text {int }}$ is not accurate enough for temperature calibration (errors over $50 \%$ can be expected). Furthermore the assumed linear relationship for $T_{i n t}$ with the resistance introduces another error as is shown in Figure 2.10. For probe temperatures up to $\sim 350{ }^{\circ} \mathrm{C}$ the data is fitted better with a quadratic function.

Using the isothermal calibration method the whole cantilever is heated via a temperature controlled hot stage followed by the measurement of the cantilever resistance as a function of temperature. Since with this method the whole cantilever is heated, the cantilever legs contribute to the measured increase in cantilever resistance for increases in temperature. Hence for a given power the resistively heated cantilever $T_{H}$ is underestimated by $\sim 10-15 \%$ when compared to Raman thermometry measurements of the same resistively heated AFM cantilevers (Figure 2.11). 


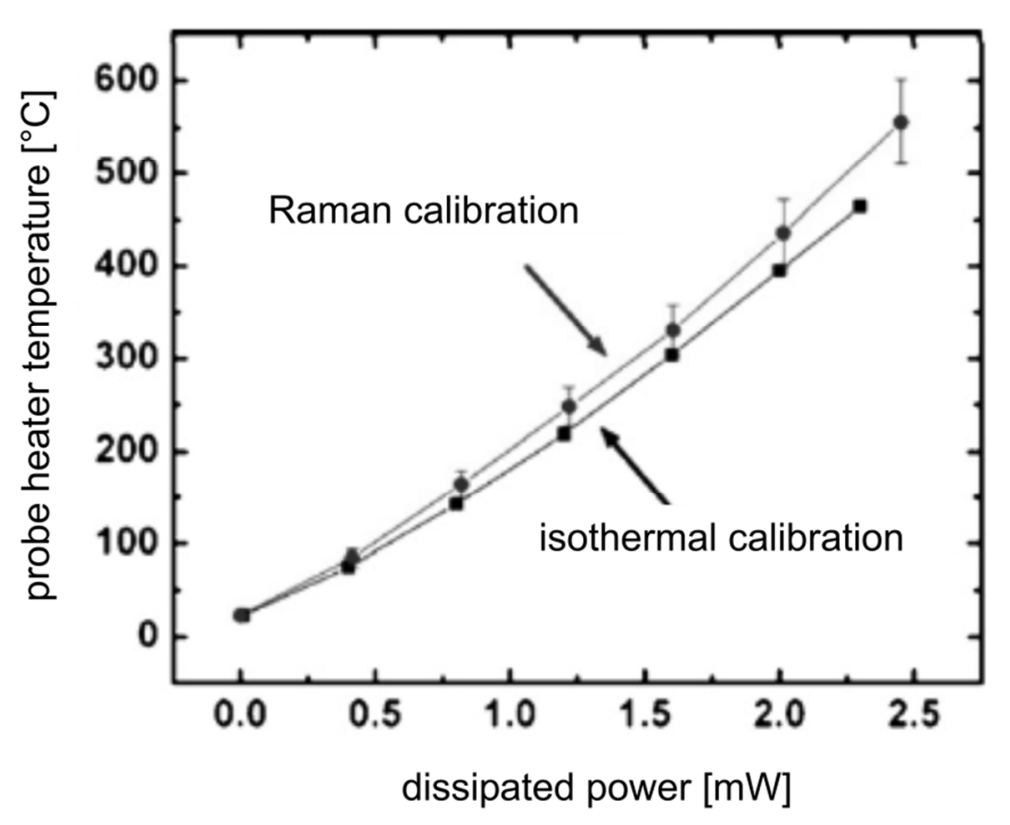

Figure 2.11: Comparison of $T_{H}$ versus dissipated power for a resistively heated silicon cantilever calibrated with Raman thermometry and the isothermal calibration method. Reprinted from reference 110, with permission from Elsevier.

Raman thermometry ${ }^{75,112}$ uses the position of the Stokes peak, the width of the Stokes peak or the ratio of the Stokes to anti-Stokes peaks to measure the temperature of a surface. The latter two quantities provide absolute temperatures. However, they require longer accumulation times to compensate for statistical fluctuations and to resolve the weak anti-Stokes peak. Temperature measurements based on the Stokes peak position are accurate and relatively fast. Measurement of the Stokes peak position as a function of the applied voltage for a heated AFM cantilever can be used to calculate the temperature according to: ${ }^{113}$

$T=T_{0}+\left(\frac{d \Omega}{d T}\right)^{-1}\left(\Omega-\Omega_{0}\right)$

In which $\Omega$ is the Stokes peak position and $d \Omega / d T$ the slope obtained from a Stokes peak position versus temperature calibration curve obtained for a piece of silicon with the same dopant concentration as the cantilever material. Typical values of $d \Omega / d T$ for the silicon AFM cantilevers are $\sim-0.0232 \mathrm{~cm}^{-1}{ }^{\circ} \mathrm{C}^{-1} \cdot{ }^{112} T_{0}$ is room temperature and $\Omega_{0}$ is the Stokes peak position for silicon at room temperature $\left(\sim 520 \mathrm{~cm}^{-1}\right)$. A disadvantage of this method is that the Stokes peak position for silicon is known to be affected by internal stresses in the material. ${ }^{112}$ In addition this method is diffraction limited which implies that averaged temperatures are measured over relatively large areas $\left(\sim 1 \mu \mathrm{m}^{2}\right)$. Hence, the sharp tip apex temperature cannot be 
resolved with Raman thermometry. Furthermore this method requires a more complicated setup; a Raman microscope setup has to be combined with the AFM setup for simultaneous operation.

Using the polymer melting point standards ${ }^{114}$ method $T_{i}$ is calibrated through the correlation of the measured heating voltages at which the known melting transitions of the polymer standard materials occur. Materials frequently used are poly( $\varepsilon$-caprolactone) (PCL), poly(ethylene) (PE) and poly(ethylene terephthalate) (PET) with melting points $\left(T_{m}\right)$ of $55{ }^{\circ} \mathrm{C}, 116{ }^{\circ} \mathrm{C}$ and $235{ }^{\circ} \mathrm{C}$, respectively. The melting point transitions are measured as follows. The cantilever is brought in contact with the polymer standard material. The force feedback of the AFM is switched off, followed by the initiation of a voltage ramp over the cantilever legs $\left(\sim 0.3 \mathrm{~V} \mathrm{~s}^{-1}\right)$. The polymer sample in close proximity to the probe tip-sample contact interface is heated and as a consequence it thermally expands. Hence the cantilever is pushed upwards, which is measured as an increase in cantilever deflection. Upon reaching the melting point transition of the polymer, the sharp probe tip penetrates the softened polymer, which is observed as a sharp drop in the measured cantilever deflection. ${ }^{84}$ The voltage, at which the slope of the deflection versus heating voltages equals 0 , is correlated to the known melting point of the polymer standard material $\left(T_{m}=T_{\mathrm{i}}\right)$ (Figure 2.12). These kinds of measurements are generally referred to as nanoscale thermal analysis (NanoTA) measurements. Due to the non-linear relationship of resistance with temperature for the resistively heated silicon AFM cantilevers a minimum of three polymer standard materials is required for construction of the temperature calibration curve.
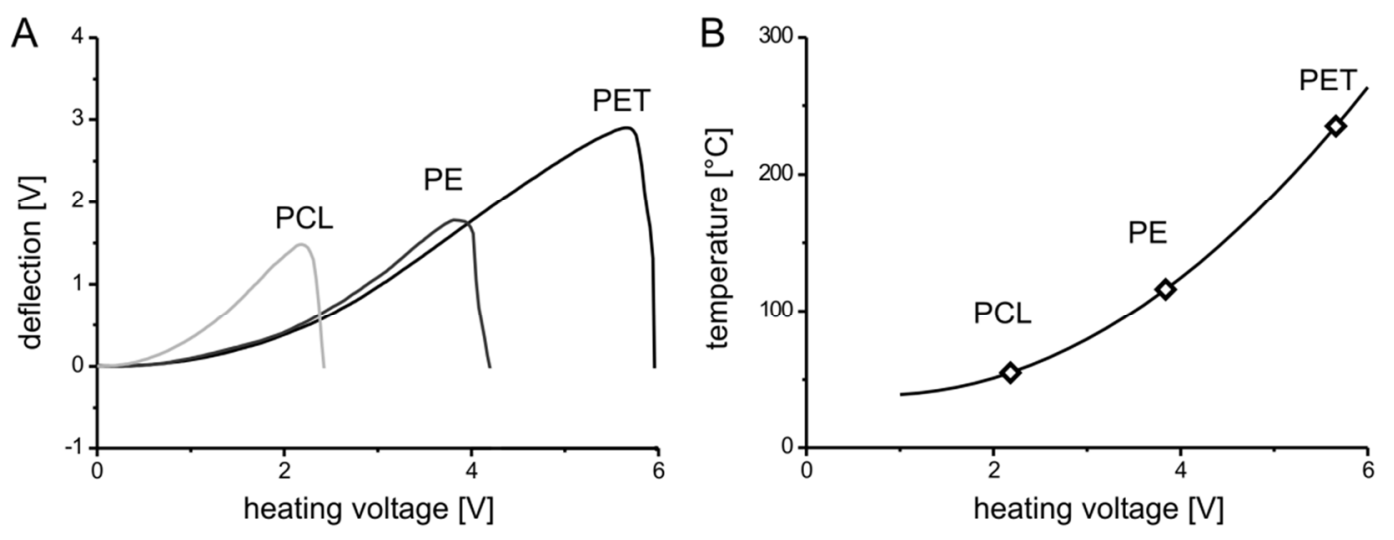

Figure 2.12: Deflection versus heating voltage for an AN2-200 cantilever on PCL, PE and $P E T$, respectively $(A)$. The voltage ramp rate used was $0.3 \mathrm{~V} \mathrm{~s}^{-1}$. The constructed $T_{i}$ calibration curve is shown in $(B)$. 
This method is relatively fast and it is the only known method available today that calibrates $T_{i}$. Critical remarks for this method are the known temperature ramp rate dependency of melting point transition in analogy with differential calorimetry measurements. ${ }^{115}$ Therefore it requires statement of the temperature ramp rate used for the measured transition temperature. For example, the melting point transition for PET was determined from NanoTA curves to be $242{ }^{\circ} \mathrm{C}, 244{ }^{\circ} \mathrm{C}$ and $249{ }^{\circ} \mathrm{C}$ for probe tip heating rates of $2{ }^{\circ} \mathrm{C} \mathrm{s}^{-1}, 5{ }^{\circ} \mathrm{C} \mathrm{s}^{-1}$ and $10{ }^{\circ} \mathrm{C} \mathrm{s}^{-1}$, respectively. Additionally the pressure applied by the heated AFM probe tip increases significantly with increasing temperature as is obvious from the increase in deflection signal. The pressure dependency of polymer transition temperatures is a known phenomenon. ${ }^{116,117}$ However estimation of the tip pressure dependent PET melting point transition revealed that from maximum possible deflection (highest load) to minimized total deflection at the melting point transition ${ }^{118}$ the melting point varied within $10{ }^{\circ} \mathrm{C}$ without a clear trend. It appeared that for increasing probe tip pressure a decrease in the measured PET melting temperature was observed, whereas for PET an increase in melting temperature with increasing pressure was expected. ${ }^{119}$ Taking into account the relatively large errors for the polymer melting point standard calibration method (approximately $\pm 10^{\circ} \mathrm{C}$ ) it is expected that the pressure effect can be ignored. When one is interested in comparing different materials with each other, best would be to minimize the cantilever deflection increase before reaching the melting point transition. ${ }^{118}$ Hence the total pressure applied by the probe tip is as low as possible. In addition the contribution of pressure effects on the error in calibrated $T_{i}$ is significantly reduced.

Another point of concern for the validity of the calibrated $T_{i}$ is the expected tip wear for silicon tips due to tip degradation or fouling. ${ }^{120}$ Both will increase the tip radius significantly. Although it was calculated by Nelson and King ${ }^{121,122}$ that only about $0.1 \%$ of the total power generated at the cantilever heater element ends up in the polymer film through the probe tip-sample interface, its contribution to the local temperature increase in proximity of the tip contact point is significant. Hence, slight alterations in tip radius of curvature will cause a severe error in the calibrated $T_{i}$. Typically it was observed for one day of operation with the same probe, in which the tip radius of curvature increased from $\sim 40 \mathrm{~nm}$ to $\sim 100 \mathrm{~nm}$ (See Chapter 8), that the measured melting point of the PET calibration sample increased with over $25{ }^{\circ} \mathrm{C}$. Therefore an appropriate validation of the accuracy of the calibrated $T_{i}$ with the polymer melting point standards is required after intensively using the same probe tip. 


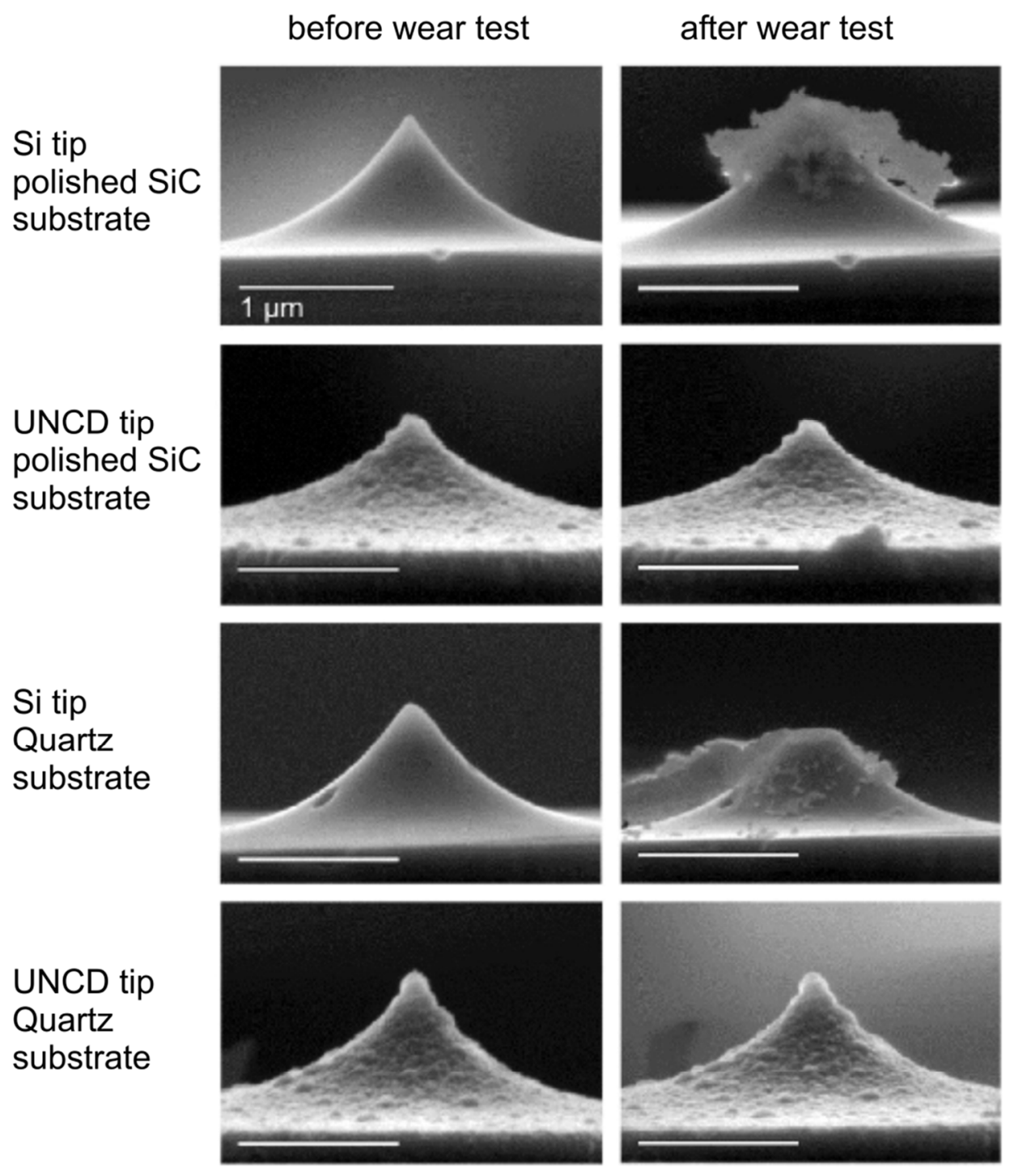

Figure 2.13: SEM images of silicon and UNCD probe tips on silicon AFM cantilevers with resistive Joule heaters embedded at the cantilever end, before and after wear tests at polished SiC and quartz substrates. For the wear test the probes were heated to $400{ }^{\circ} \mathrm{C}$ and a contact load of $\sim 200 \mathrm{nN}$ was applied. Reprinted with permission from reference 128. Copyright 2010 American Chemical Society.

One attempt to prevent tip wear effects for the resistively heated silicon probe tips has been reported on by Vettiger and coworkers. ${ }^{123}$ They attached 150 - $300 \mathrm{~nm}$ long multiwalled carbon nanotube (MWCNT) tips (diameter $\sim 20 \mathrm{~nm}$ ) to the silicon probe tip on a cantilever with integrated resistive heater element. CNT tips are known to be robust and even if tip wear would occur it only affects the length of the tip, not the diameter of the CNT. ${ }^{124,125}$ Additionally, the high thermal conductivity of CNTs ${ }^{126,127}$ make them attractive for utilization as tip material for heated AFM cantilevers. Prolonged heating above $550{ }^{\circ} \mathrm{C}$ in air resulted in significant decreases in length of the MWCNT tips without affecting their diameter. More recently, King and 
coworkers ${ }^{128}$ have reported on the fabrication of wear resistant ultrananocrystalline diamond (UNCD) probe tips attached to silicon cantilevers with resistive heaters embedded at the cantilever end. The tip radius of curvature of the prepared diamond tips was $\sim 50 \mathrm{~nm}$, which is slightly larger than typically reported on for the sharpened silicon tips (AN2-type). Figure 2.13 shows SEM images of silicon and diamond tips before and after scanning polished $\mathrm{SiC}$ and quartz surfaces with heated probe temperatures of $400{ }^{\circ} \mathrm{C}$ and a contact load of $200 \mathrm{nN}$. The used conditions for the wear test are quite harsh compared to the typical conditions used for SThL throughout this Thesis (maximum $T_{i} 300{ }^{\circ} \mathrm{C}$, maximum load $<50 \mathrm{nN}$ ). Overall it was shown that the silicon tips had a 100 fold higher wear rate (measured in $\mathrm{m}^{3} \mathrm{~N}^{-1} \mathrm{~m}^{-1}$ ) compared to the UNCD tips. In order to place the effects of tip wear on calibrated $T_{i}$ values in the right perspective the modes of heat transport from heated silicon AFM cantilevers in contact with a substrate will be discussed next.

\subsection{Heat transport from resistively heated AFM cantilevers}

Recently Butt and coworkers ${ }^{129}$ introduced an optical detection setup for surface temperature measurements with microsecond and microscale resolution. Their setup was originally built to detect the thermal emission spectrum of a polymer film heated by a laser in the visible range. Fitting the emission spectrum to Planck's law a 2D temperature profile was obtained. Within the same paper they extended their setup to measure the 2D temperature profile of a heated silicon AFM cantilever (Figure 2.14).
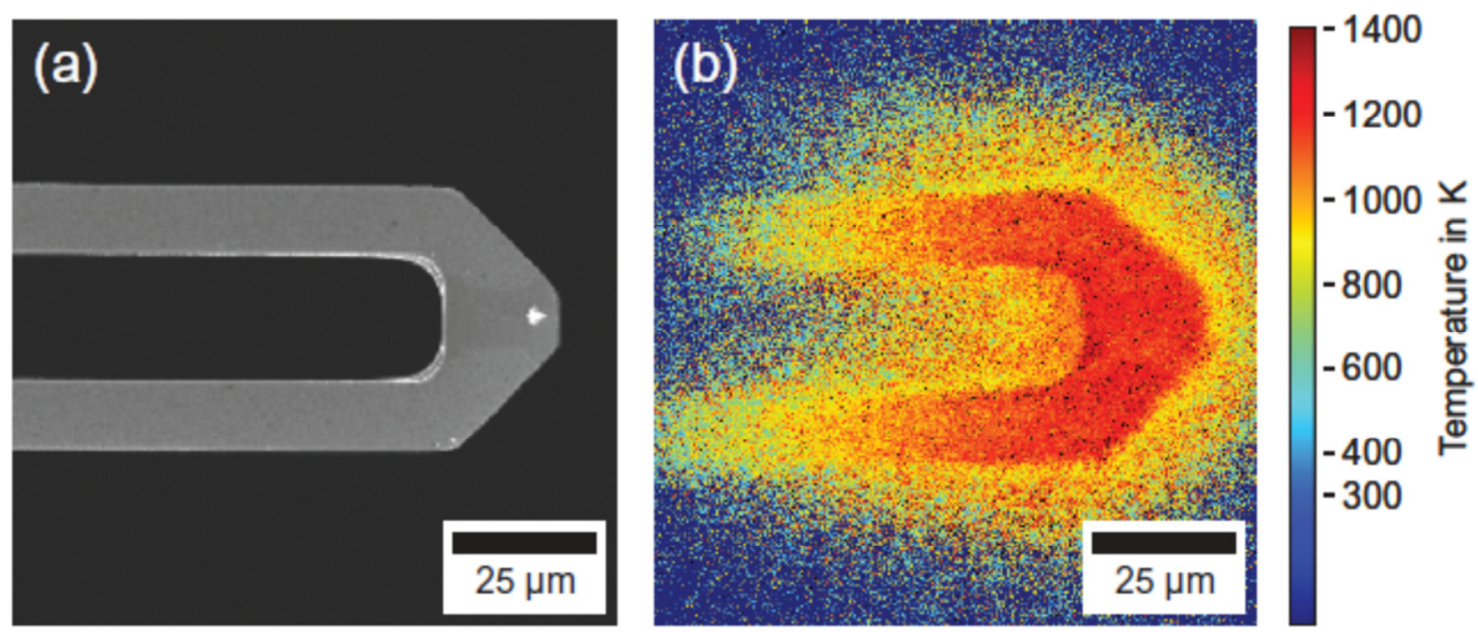

Figure 2.14: SEM image of an AN2-200 cantilever with resistive heater element integrated in the cantilever end (A). $2 D$ temperature distribution of the heated cantilever $(4.5 \mathrm{~V}, 8.31 \mathrm{~mA})$ (B). Adapted from reference 129. With kind permission from IOP Publishing Ltd. 
Figure 2.14 clearly shows that the heat generated in the resistive Joule heater area, embedded at the cantilever end, remains highly localized. Temperatures at the cantilever end are approximately $900{ }^{\circ} \mathrm{C}$. Within distances of $\sim 70 \mu \mathrm{m}$ from the cantilever end the temperature dropped to values that were too low to be resolved with this experimental setup. Furthermore it was shown that most of the heat generated at the cantilever end flows into the cantilever legs (high thermally conductive silicon), from where most of the heat is conducted into the surrounding air. This is in agreement with numbers reported on by King and coworkers. ${ }^{121,122,130}$ They reported that approximately $80 \%$ of the power generated in the cantilever end flows via the cantilever legs into the substrate underneath the heated cantilever, whereas only $0.1 \%$ is conducted through the heated probe tip-sample interface. The uniform temperature distribution, noticed in Figure 2.14 at the cantilever end when positioned in air, indicates that the temperature of the tip is not significantly different from the cantilever itself. However the spatial resolution of the optical temperature measurement setup is $\sim 1 \mu \mathrm{m}$ and hence the tip apex can not be resolved.

Next we will examine the situation in which a heated AFM cantilever is brought into contact with a substrate (e.g. a polymer film) (Figure 2.15).

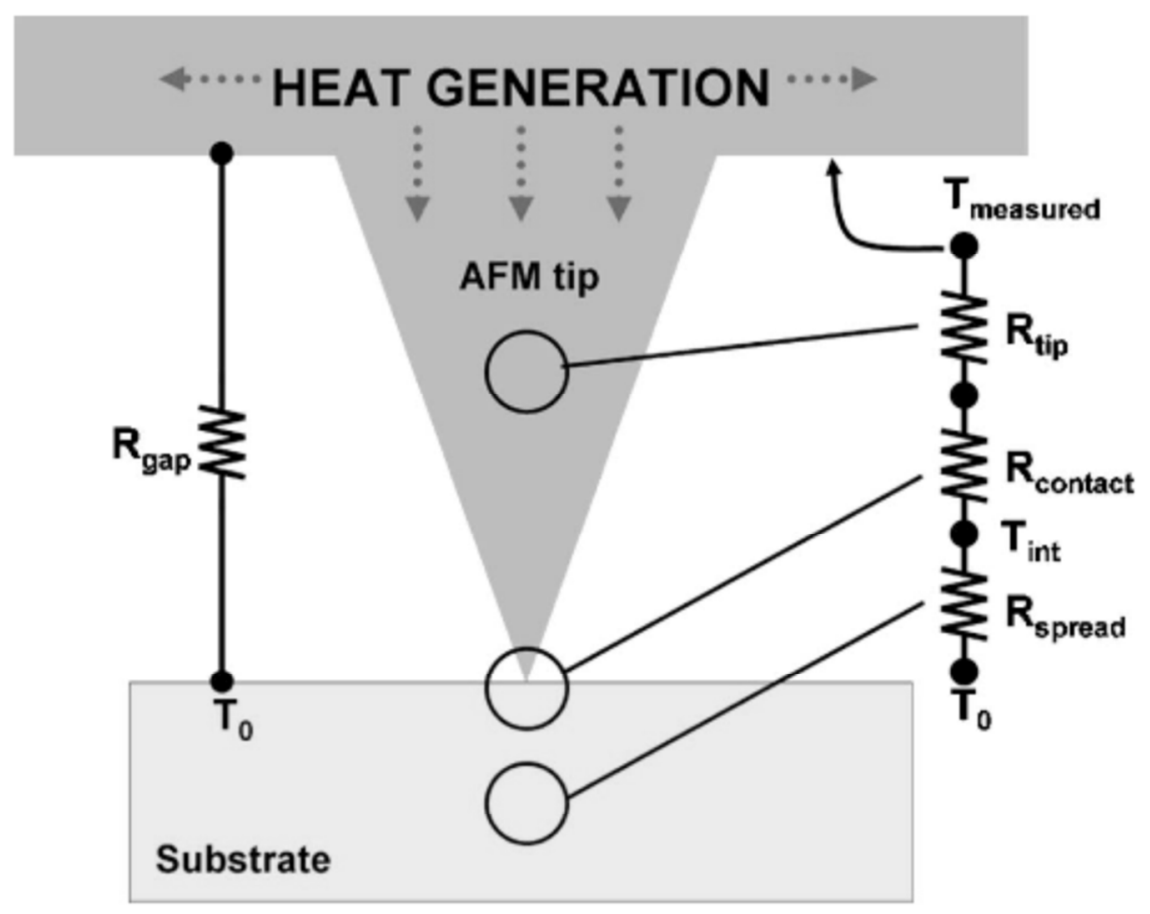

Figure 2.15: Thermal circuit for heat flow through the tip of a heated AFM cantilever. The relative sizes of the thermal resistances $(R)$ of the tip $\left(R_{\text {tip }}\right)$, probe tip-sample contact interface $\left(R_{\text {contact }}\right)$ and substrate $\left(R_{\text {spread }}\right)$, determine the interface temperature $\left(T_{\text {int }}=T_{i}\right)$. The dominant mode of heat transport is conduction through the air gap, which has a thermal resistance of $R_{\text {gap }}$. Reprinted with permission from reference 122. Copyright 2007, American Institute of Physics. 
This model treats heat transport in terms of thermal resistances $(R)$. The thermal resistance of a material is defined as:

$$
R=\frac{\Delta T}{Q}
$$

In which $\Delta T\left({ }^{\circ} \mathrm{C}\right)$ is the temperature drop over the corresponding layers with heat flow $Q(\mathrm{~W})$.

Since most of the generated power is transported over the air gap into the substrate, $R_{\text {gap }}$ is relatively low. However the temperature rise on the substrate surface due to heat transfer over the air gap is controlled by the ratio of the thermal conductivities of air and the substrate. Hence, the increase in substrate temperature below the heated cantilever is relatively low compared to polymer-silicon tip contacts. Compared to the contribution of $R_{\text {contact }}$, the contribution of $R_{\text {tip }}$ is negligible. The former is argued to be dominated by phonon scattering at the heated probe tip-sample interface. ${ }^{131,132}$ For a heated tip in contact with a polymer film typical values of $R_{\text {tip }}$ and $R_{\text {contact }}$ are $10^{6} \mathrm{~K} \mathrm{~W}^{-1}$ and $10^{7} \mathrm{~K} \mathrm{~W}^{-1}$, respectively. ${ }^{122}$

Nelson and King ${ }^{131}$ used this thermal resistance model to model the interface temperature of a heated probe in contact with a polymer or silicon substrate at different contact loads. Taking into account the substrate thermal conductivity as well as probe tip sample contact area, differences in $T_{i}$ for a probe tip in contact with the relatively soft polymer compared to the hard silicon surface were calculated. Their results are shown in Figure 2.16.

From Figure 2.16 it is clear that good thermally conductive substrates (high thermal conductivity ratio) significantly reduce $T_{i}$. Furthermore it is clear that for increasing the contact load $T_{i}$ increases for samples with the same thermal conductivity. This is related to an increase in tip-sample contact area, significantly reducing $R_{t i p}$. For harder substrates this effect is less pronounced, as expected. The effects of tip wear as discussed earlier on the calibrated $T_{i}$ are underlined with these calculations. 
A

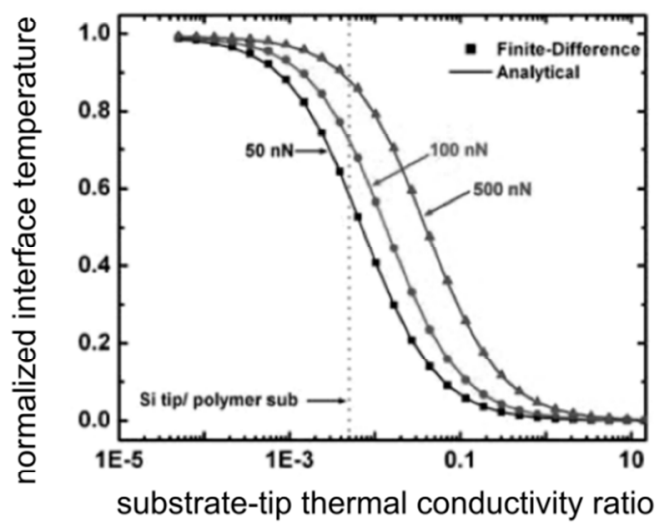

B

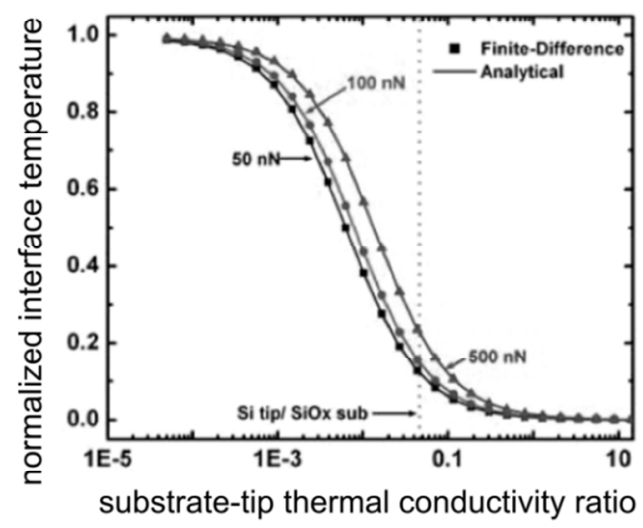

Figure 2.16: Normalized $T_{i}$ as a function of the ratio between the tip and substrate thermal conductivities for a soft polymer $(A)$ and a hard silicon substrate $(B)$ in contact with a heated probe tip with different contact loads. The vertical dashed lines represent the substrate-tip thermal conductivity ratios for polymeric and silicon substrates, respectively. Reprinted with permission from reference 131.

Besides thermal conduction, other paths of heat transfer are radiation and convection. Radiative heat transfer from the heated silicon cantilevers $\left(T_{H} \sim 400{ }^{\circ} \mathrm{C}\right)$ was calculated to be less than $1 \%$ of the total input power (order $\mathrm{mW}$ ) with the Stefan-Boltzmann law ${ }^{133}$, assuming that the cantilever is a black body. Hence thermal radiation can be neglected. King and coworkers ${ }^{95}$ calculated that the contribution of convection from the cantilever was even smaller than radiative heat transport. This is in agreement with the general conception that buoyancy forces are smaller than viscous forces for microscale heat sources. ${ }^{134,135}$ Hence convection from the heated cantilever can also be ignored. This leaves conduction as discussed above as the main heat transport mode.

Taking the discussed heat transport modes into account for the calibration of $T_{i}$ (discussed above) it can be concluded that there is no golden standard method available today. The use of polymer standards seems to be justified in terms of calibrating $T_{i}$, taking the presence of the polymer into account for the temperature distribution within the heated probe tip. However, when using polymer melting standards, the effect of substrate thermal conductivity can not be ignored, when examining surfaces other than polymers. For our application (i.e. SThL), where polymer films will be used, this effect is expected to be insignificant, since most of the polymer thermal conductivities are in a quite narrow range. Decreasing the films thickness to very low values is expected to affect $T_{i}$ when very poor or very good thermal conductive substrates are used. Chapter 4 provides some pioneering work in which the polymer film thickness effect on the measured surface softening 
temperature is discussed in terms of an error introduced to the calibrated $T_{i}$ based on thick polymer melting point standard samples.

\subsection{Scanning thermal lithography}

Besides SThL, the use of heated AFM probes has been reported among others for (nanoscale) thermal analysis (NanoTA), ${ }^{10,122,136-138}$ thermal cantilever actuation, ${ }^{139-141}$ on cantilever processes, ${ }^{142,143} \mathrm{SThM}^{9,144,145}$ and topography imaging. ${ }^{77,146,147}$ These applications were nicely reviewed by Nelson and $\mathrm{King}^{87}$ and are therefore not further described here. This section will focus on SThL.

The first example of SThL was the thermomechanical indentation of a polymer film with a laser heated AFM probe to produce data bits. ${ }^{72}$ The great efforts of the IBM research teams involved in the Millipede project ${ }^{148}$ have brought this approach to a relatively mature level. Indents smaller than $40 \mathrm{~nm}$ can be produced in PMMA films with data storage densities exceeding $1 \mathrm{Tbit}_{\text {inch }}{ }^{-2} \cdot{ }^{66,148}$ Besides the thermomechanical material shaping, three more modes for heated probe assisted maskless (nano-) lithography have been identified: i) material deposition, ii) material removal and iii) thermochemical conversion.
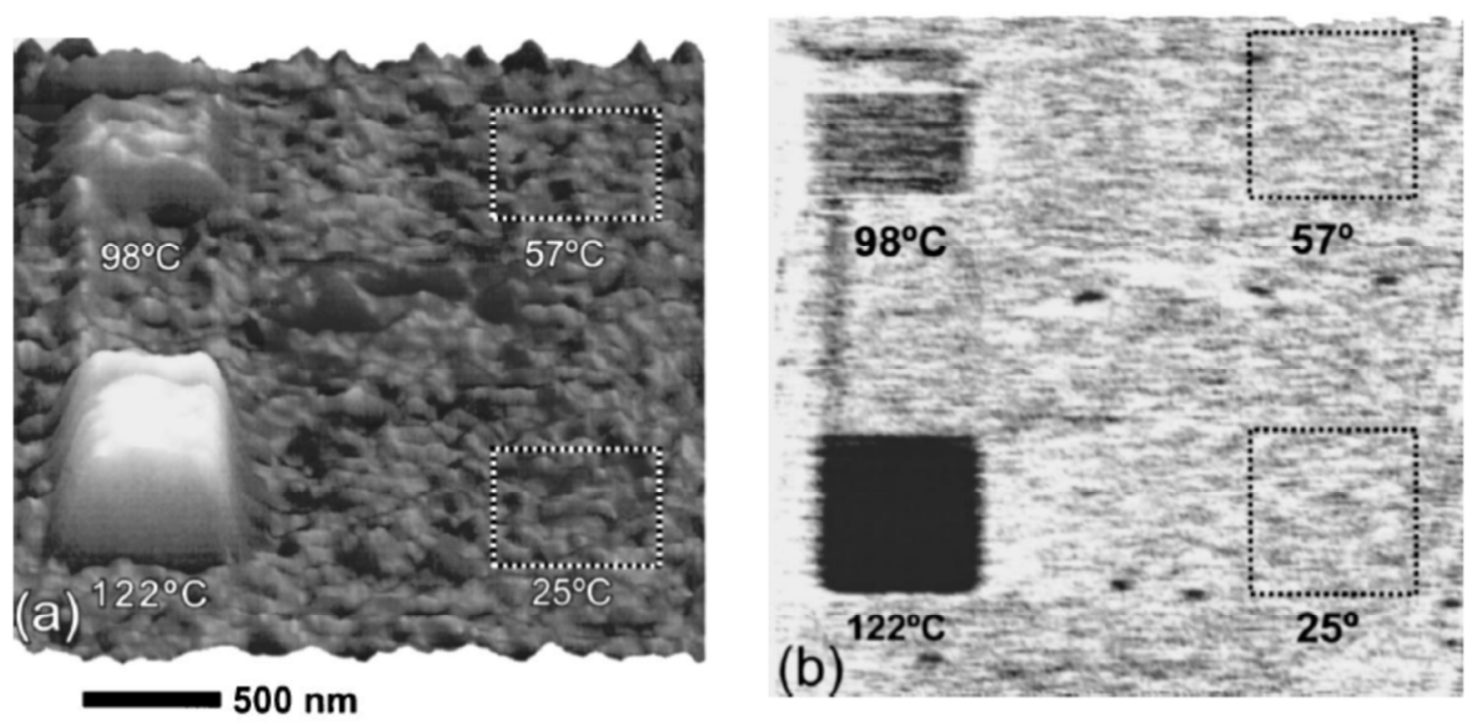

Figure 2.17: AFM height (A) and lateral force (B) images of four squares (500 nm $\times 500 \mathrm{~nm}$ ) written with an OPA coated tip by thermal Dip-Pen NL at different probe temperatures. Obviously, at probe temperatures below the melting point of OPA $\left(\sim 100^{\circ} \mathrm{C}\right)$ no material was deposited. Reprinted with permission from reference 149. Copyright 2004, American Institute of Physics. 
Thermally assisted material deposition has been introduced in 2004 by King and coworkers. ${ }^{149}$ They reported on the deposition of octadecylphosphonic acid (OPA) on mica surfaces. They referred to this method as thermal Dip-Pen NL. The ink used in thermal Dip-Pen NL is solid at room temperature; hence no material is deposited while scanning with the ink coated tip at this temperature. Upon heating above the melting point of OPA $\left(T_{m} \sim 100{ }^{\circ} \mathrm{C}\right)$ material flows from the tip to the substrate and material is deposited (Figure 2.17). The main advantage of this method over Dip-Pen NL as introduced by the Mirkin group ${ }^{52}$ is thus the prevention of surface contamination. Furthermore the writing speed can be increased with increasing the probe tip temperature. A disadvantage is the poor resolution of this technique $(\sim 80 \mathrm{~nm})$ compared to results reported on for Dip-Pen NL. ${ }^{52,150}$ Other examples of thermal Dip-Pen NL are the deposition of indium on glass and silicon surfaces ${ }^{151}$ and the controlled deposition of stimulus responsive poly( $\mathrm{N}$-isopropylacrylamide) onto silicon surfaces. ${ }^{152}$
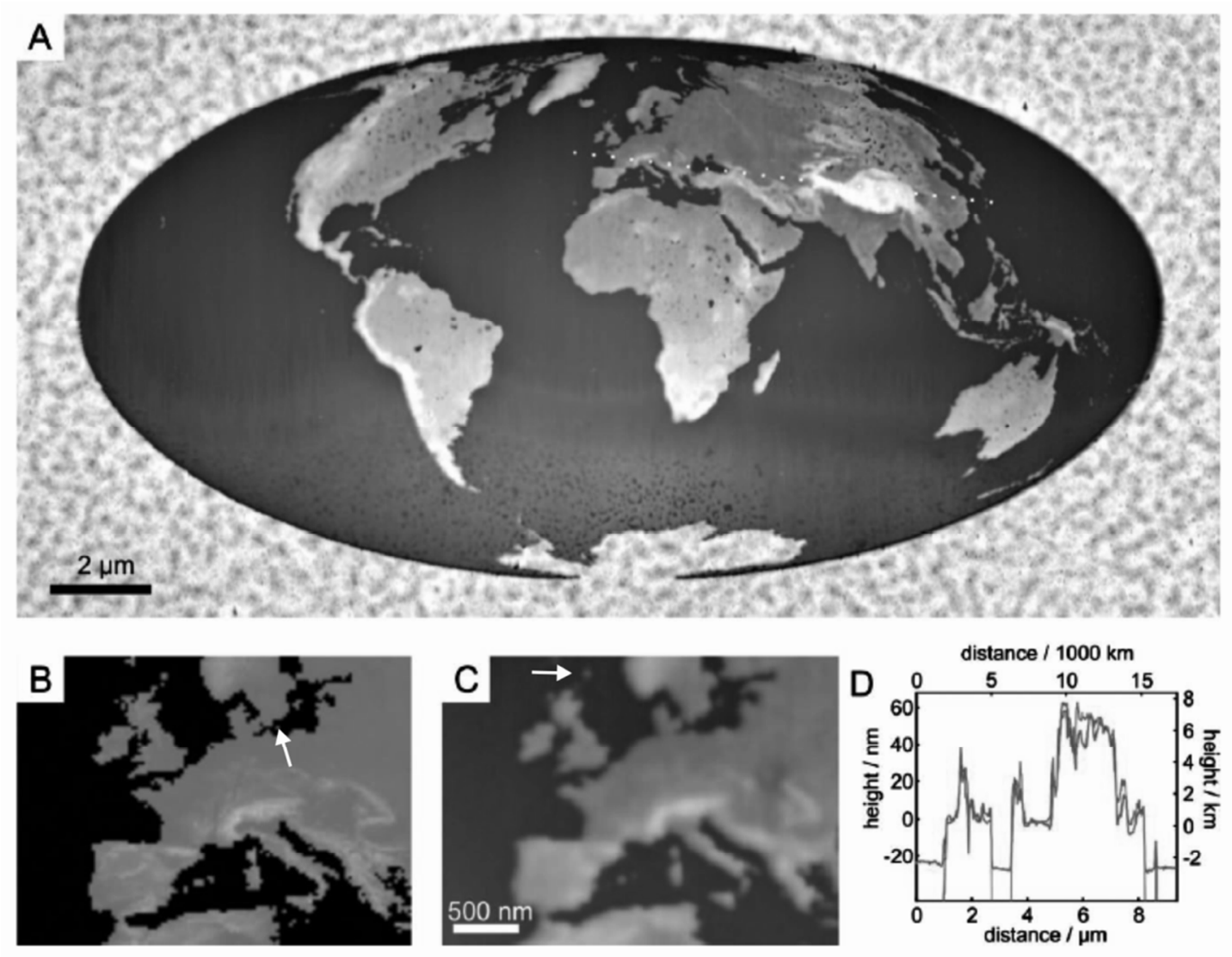

Figure 2.18: AFM topography image of a $3 D$ world map written into a $250 \mathrm{~nm}$ thick poly (phthalaldehyde) layer (A). The total pattern consists of $1 \times 10^{5}$ pixels and was prepared within 143 seconds. In (B) a sub area of the programmed bitmap is shown and in (C) the imaged relief. The white arrows indicate positions with features of 1 (in $B$ ) and 2 (in $C$ ) pixels with the original bitmap. It shows that a minimum number of 2 pixels were needed for correct reproduction. The corresponding cross-section along the white dashed line in $(A)$ is shown in (D) and compared to the original topography line in the bitmap. The Alps, Black Sea, Caucasian mountains and Himalayas are resolved. Adapted from reference 155. 
In contrast to material deposition, heated probes can also be used for the selective removal of material. King and coworkers have reported on the thermal decomposition of poly(hydroxyl styrene) and PMMA films. ${ }^{153,154}$ The amount of material removed can easily be controlled by adjusting the heated probe temperature as well as the writing speed. Writing speeds up to $60 \mu \mathrm{m} \mathrm{s}^{-1}$ were achieved. Very recent work from Knoll and coworkers have demonstrated the 3D patterning of polymer films with heated AFM probe cantilevers at $\sim 40 \mathrm{~nm}$ lateral and $\sim 1 \mathrm{~nm}$ vertical resolution at high speed. ${ }^{155-157}$ Their most outstanding example is shown in Figure 2.18. This 3D replica of the world map in a custom designed $250 \mathrm{~nm}$ thick poly(phthalaldehyde) film was prepared in only 143 seconds and contains $5 \times 10^{5}$ pixels!! Vertical 3D pattern amplification of a factor 6 was achieved with a $\mathrm{SF}_{6}$ reactive ion etch process.

Examples of thermal chemical material conversion with heated AFM probes are the spatially controlled localized crosslinking of a photoresist (AZ5214E, Clariant Corp.) with the ultracompliant polyimide probes (described in Section 2.3) reported on by Gianchandani and coworkers. ${ }^{158}$ Line widths of $\sim 500 \mathrm{~nm}$ were obtained. Increasing the resolution for this approach was reported to be straightforward, i.e. use sharper tips, thinner resist layers and potentially change to chemically amplified resists. In fact, Cacialli and coworkers ${ }^{159}$ have demonstrated the $\sim 30 \mathrm{~nm}$ patterning of an organic semiconductor precursor with a heated Wollaston wire with a diameter of $\sim 5$ um. With a contact diameter of approximately $100 \mathrm{~nm}$, poly(p-xylene tetrahydrothiophenium chloride) polymer precursor films were locally thermally converted into fully conjugated poly( $p$-phenylene vinylene) (PPV) at wire temperatures of approximately $200{ }^{\circ} \mathrm{C}$. This example was the first successful example that used a heated wire for thermal chemical surface reactions following our example described in Chapter 7. Two months later than Cacialli and coworkers, the King group $^{160}$ reported on the same thermal chemical surface conversion by a heated silicon AFM probe with sub $100 \mathrm{~nm}$ spatial resolution. Although the contact area of the probes used by the King group is expected to be much smaller than the heated wire used by Cacialli and coworkers, the resolution achieved by the King group is much worse. This is ascribed to thermomechanical ploughing in the precursor film during SThL as is observed from the formation of up to $10 \mathrm{~nm}$ deep grooves and material pile up at the end of lines written with the heated silicon AFM cantilevers.

The corresponding fluorescence microscopy images revealed little background fluorescence which is in agreement with the fact that most of the heat is localized in the proximity of the heated probe tip contact point (i.e. spatially a sharp temperature drop is expected). Hence sub $100 \mathrm{~nm}$ resolutions for this technique are expected. 
So far the applications of these heated AFM probes have been restricted to the use in semiconductor or mask fabrication processes. However after the start of this project the King group ${ }^{68,161}$ and our group ${ }^{69}$ (See Chapter 7) have reported on the spatially controlled local thermal chemical surface functionalization for further wet chemical derivatization. King and coworkers ${ }^{68}$ reported on the sub $15 \mathrm{~nm}$ thermal chemical deprotection of tetrahydropyranyl protected surface exposed esters in tailor made crosslinked polymer films consisting of poly(tetrahydro- $2 H$-pyran-2-yl methacrylate $)_{80} \quad-\quad \operatorname{poly}(3-\{4-[(E)-3-$ methoxy-3-oxoprop-1-enyl $]$ phenoxy $\}$ propyl-2methacrylate $)_{20}\left(\mathrm{p}(\mathrm{THP}-\mathrm{MA})_{80} \mathrm{p}(\mathrm{PMC}-\mathrm{MA})_{20}\right.$ ). However within this report the surface derivatization was not covalent and their results for sub $15 \mathrm{~nm}$ surface functionalization were not very convincing (Figure 2.19). The observed pile up of material surrounding the line patterns shown in Figure 2.19, point towards thermal mechanical pattern formation instead of towards a thermochemical surface transition.
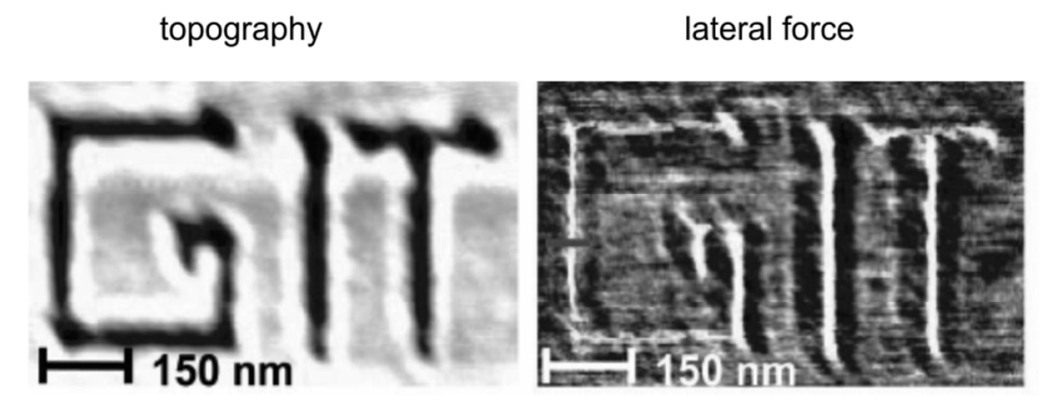
cross section lateral force

Figure 2.19: AFM topography and lateral force images of the letters GIT written with SThL in a $p(\text { THP-MA })_{80} p(P M C-M A)_{20}$ polymer film. The lateral force cross-sectional analysis implies sub 15 nanometer thermal chemical conversion. Reprinted with permission from reference 68. Copyright 2007 American Chemical Society.

In addition we reported on the thermochemical functionalization of $370 \mathrm{~nm}$ $\times 580 \mathrm{~nm}$ domains in poly(styrene)-block-poly(tert-butyl acrylate) (PS- $b$-P $t$ BA) polymer films followed by the wet chemical grafting of fluoresceinamine (See Chapter 7). ${ }^{69}$ The observed domains were a combination of thermomechanical deformations as a result of the probe tip temperature being much higher than the glass transition temperature of the block copolymer and thermochemical functionalization as a result of the thermolysis of the tert-butyl ester protected carboxylic acid groups.

In more recent work King and coworkers ${ }^{162}$ reported on the thermal chemical surface functionalization with SThL on a polymer film containing surface exposed tetrahydropyranyl carbamate groups. Upon heating the polymer to temperatures between $150{ }^{\circ} \mathrm{C}$ to $200{ }^{\circ} \mathrm{C}$ the tetrahydropyranyl carbamate groups were thermally cleaved off to unmask primary amine groups. The latter are known as chemically 
active moieties for controlled modification with interesting biomolecules or other ligands. Multiple functionality patterns were prepared via the controlled introduction of biotins, thiols and amines in multiple step processes. Dots $(\sim 100 \mathrm{~nm}$ diameter $)$ chemically activated with SThL (Figure 2.20) were conjugated with fibronectin molecules. The size and shape of the proteins present after conjugation in the dot areas indicate that only a few protein molecules were surface bound to these areas. These results clearly demonstrate the high resolution achievable with SThL for thermal chemical surface functionalization and subsequent aqueous derivatization.

After SThL
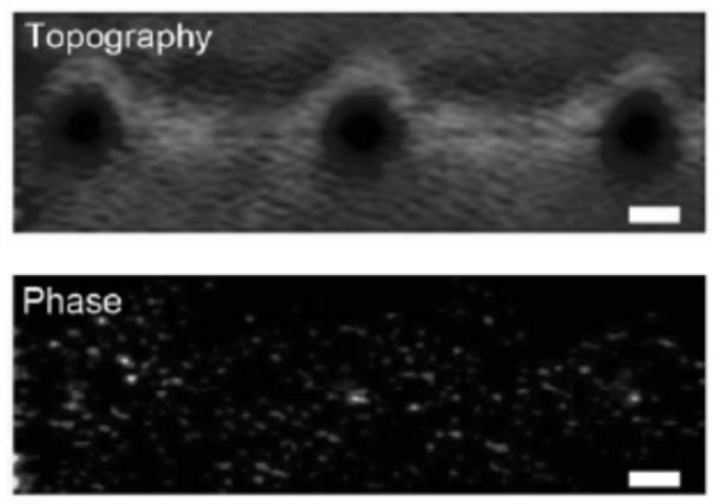

After Fibronectin Conjugation
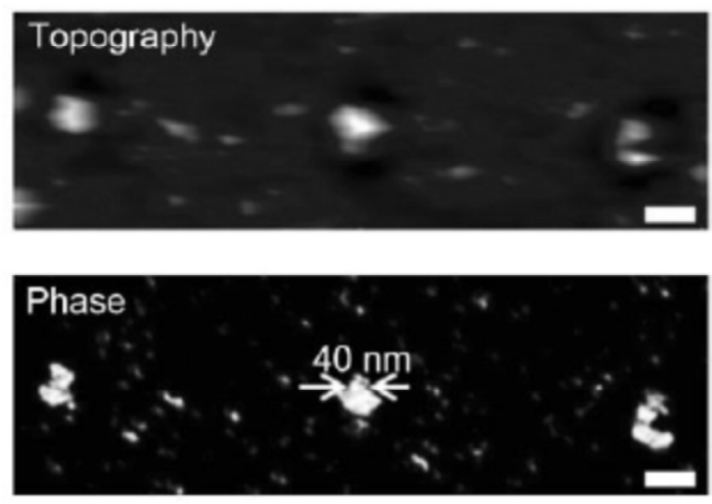

Figure 2.20: AFM height (top) and phase (bottom) images of selectively amine functionalized areas with SThL (left) followed by the selective conjugation with fibronectin (right). The scale bars correspond to $100 \mathrm{~nm}$. Adapted from reference 162.

\subsection{Conclusions}

The development of batch fabricated AFM cantilevers with integrated resistive Joule heaters at the cantilever end and silicon tips with apexes as sharp as $20 \mathrm{~nm}$ allowed SThM to evolve into an established nanotechnology tool for the among others sub $100 \mathrm{~nm}$ spatial resolution thermal property imaging, NanoTA and SThL. Especially thermochemical surface modification with SThL is a young and exciting field for the development of chemically patterned surfaces with a large diversity of chemical functionalities with nanoscale spatial resolution. Parts of the contributions are described in this Thesis. Despite the reported sub 100 nanometer thermochemical patterning resolution for this approach and its relatively high $\left(>>5 \mu \mathrm{m} \mathrm{s}^{-1}\right)$ patterning speed more research is needed for it to grow into a mature tool available for rapid prototyping of e.g. biosensors. For instance, the diversity of the chemical functionalities embedded in especially designed polymer film platforms, that could be thermochemically deprotected, should be increased in order to reduce the number of processing steps compared to the multi-step processes reported on so far. 


\subsection{References and notes}

1 Binnig, G.; Rohrer, H.; Gerber, C.; Weibel, E. Phys. Rev. Lett. 1982, 49, 57.

2 Binnig, G.; Quate, C. F.; Gerber, C. Phys. Rev. Lett. 1986, 56, 930.

3 Hofer, W. A.; Foster, A. S.; Shluger, A. L. Rev. Mod. Phys. 2003, 75, 1287.

4 Bai, C. Scanning tunneling microscopy and its applications, $2^{\text {nd }}$ ed.,Springer Verlag, New York, 2000.

5 Evans, E. Annu. Rev. Biophys. Biomol. Struct.. 2001, 30, 105.

6 Rief, M.; Clausen-Schaumann, H.; Gaub, H. E. Nat. Struct. Biol. 1999, 6, 346.

7 Frisbie, C. D.; Rozsnyai, L. F.; Noy, A.; Wrighton, M. S.; Lieber, C. M. Science 1994, 265, 2071.

8 Noy, A.; Vezenov, D. V.; Lieber, C. M. Annu. Rev. Mater. Sci. 1997, 27, 381.

9 Majumdar, A. Annu. Rev. Mater. Sci. 1999, 29, 505.

10 Pollock, H. M.; Hammiche, A. J. Phys. D Appl. Phys. 2001, 34, R23.

11 Sahin, O.; Magonov, S.; Su, C.; Quate, C. F.; Solgaard, O. Nat. Nanotechnol. 2007, $2,507$.

12 Sahin, O.; Erina, N. Nanotechnology 2008, 19, 445717.

13 Saenz, J. J.; Garcia, N.; Grutter, P.; Meyer, E.; Heinzelmann, H.; Wiesendanger, R.; Rosenthaler, L.; Hidber, H. R.; Günterodt, H. J. J. Appl. Phys. 1987, 62, 4293.

14 Rugar, D.; Mamin, H. J.; Güthner, P.; Lambert, S. E.; Stern, J. E.; Mcfadyen, I.; Yogi, T. J. Appl. Phys. 1990, 68, 1169.

15 García, R.; Pérez, R. Surf. Sci. Rep. 2002, 47, 197.

16 Giessibl, F. J. Rev. Mod. Phys. 2003, 75, 949.

17 Magonov, S. N.; Reneker, D. H. Annu. Rev. Mater. Sci. 1997, 27, 175.

18 Loos, J. Adv. Mater. 2005, 17, 1821.

19 Jalili, N.; Laxminarayana, K. Mechatronics 2004, 14, 907.

20 Vancso, G. J.; Hillborg, H.; Schönherr, H. Adv. Polym. Sci. 2005, 182, 55.

21 Magonov, S. N. and M-H. Whangbo, Surface analysis with STM and AFM: experimental and theoretical aspects of image analysis, VCH, Weinheim 1996.

22 Morita, S. R.; Wiesendanger, R. Noncontact Atomic Force Microscopy, Nanoscience and technology. In: Nanoscience and Technology, eds: Morita, S. R.; Wiesendanger, R.; Meyer, E. Springer, Berlin 2002.

23 Miles M. J. In: Spells, S. J. editor. Characterization of solid polymers: New techniques and developments, Chapman and Hall, London, 1994, p.17.

24 Israelachvili, J. Intermolecular and Surface Forces, $2^{\text {nd }}$ ed., Academic Press, London, 1997.

25 Marti, O. In: Bushan, B. editor, Handbook of Micro/Nano Tribology, CRC Press, New York, 1999, p. 81-144.

26 Tocha, E.; Schönherr, H.; Vancso, G. J. Langmuir 2006, 22, 2340.

27 Zhong, Q.; Inniss, D.; Kjoller, K.; Elings, V. B. Surf. Sci. 1993, 290, L688.

28 Fotiadis, D.; Scheuring, S.; Müller, S. A.; Engel, A.; Müller, D. J. Micron 2002, 33, 385.

29 Putman, C. A. J.; van der Werf, K. O.; de Grooth, B. G.; van Hulst, N. F.; Greve, J. Appl. Phys. Lett. 1994, 64, 2454.

30 a) Schönherr, H. dissertation, Twente University, 1999. b) Schönherr, H.; Vancso, G. J. In: Scanning Force Microscopy of Polymers, Springer Verlag, Berlin, Vienna, 2010, p. 4.

31 Giannotti, M. I.; Vancso, G. J. Chem. Phys. Chem. 2007, 8, 2290. 
32 Ringger, M.; Hidber, H. R.; Schlogl, R.; Oelhafen, P.; Güntherodt, H. J. Appl. Phys. Lett. 1985, 46, 832 .

33 Eigler, D. M.; Schweizer, E. K. Nature 1990, 344, 524.

34 Dagata, J. A.; Schneir, J.; Harary, H. H.; Evans, C. J.; Postek, M. T.; Bennett, J. Appl. Phys. Lett. 1990, 56, 2001.

35 Krämer, S.; Fuierer, R. R.; Gorman, C. B. Chem. Rev. 2003, 103, 4367.

36 García, R.; Martinez, R. V.; Martinez, J. Chem. Soc. Rev. 2006, 35, 29.

37 Tseng, A. A.; Notargiacomo, A.; Chen, T. P. J. Vac. Sci. Technol. B 2005, 23, 877.

38 Wouters, D.; Schubert, U. S. Angew. Chem. Int. Ed. 2004, 43, 2480.

39 Xie, X. N.; Chung, H. J.; Sow, C. H.; Wee, A. T. S. Mat. Sci. Eng., R 2006, 54, 1.

40 Stievenard, D.; Legrand, B. Prog. Surf. Sci. 2006, 81, 112.

41 Nyffenegger, R. M.; Penner, R. M. Chem. Rev. 1997, 97, 1195.

42 Liu, G. Y.; Xu, S.; Qian, Y. L. Acc. Chem. Res. 2000, 33, 457.

43 Kinsella, J. M. and Ivanisevic, A. In: P. Avouris P.; Bhushan B.; von Klitzing K.; Sakaki, H.;

Wiesendanger, R. editors. Nanoscience and Technology, Springer-Verlag, Berlin, 2006, Chapter 23.

44 Soh, H. T.; Guarini, K. W.; Quate, C. F. Scanning Probe Lithography, Kluwer Academic Publishers, Boston, 2001.

45 Rosa, L. G.; Liang, J. J. Phys. Condens. Matter. 2009, 21, 483001.

46 Jung, T. A.; Moser, A.; Hug, H. J.; Brodbeck, D.; Hofer, R.; Hidber, H. R.; Schwarz, U. D. Ultramicroscopy 1992, 42, 1446.

47 Klehn, B.; Kunze, U. J. Appl. Phys. 1999, 85, 3897.

48 Kunze, U. Superlattice Microst. 2002, 31, 3.

$49 \mathrm{Xu}, \mathrm{S}$; Liu, G. Y. Langmuir 1997, 13, 127.

50 Jaschke, M.; Butt, H. J. Langmuir 1995, 11, 1061.

51 Ginger, D. S.; Zhang, H.; Mirkin, C. A. Angew. Chem. Int. Ed. 2004, 43, 30.

52 Piner, R. D.; Zhu, J.; Xu, F.; Hong, S. H.; Mirkin, C. A. Science 1999, 283, 661.

53 Lee, K. B.; Park, S. J.; Mirkin, C. A.; Smith, J. C.; Mrksich, M. Science 2002, 295, 1702.

54 Deladi, S.; Tas, N. R.; Berenschot, J. W.; Krijnen, G. J. M.; de Boer, M. J.; de Boer, J. H.; Peter, M.; Elwenspoek, M. C. Appl. Phys. Lett. 2004, 85, 5361.

55 Deladi, S.; Berenschot, J. W.; Tas, N. R.; Krijnen, G. J. M.; de Boer, J. H.; de Boer, M. J.; Elwenspoek, M. C., J. Micromech. Microeng. 2005, 15, 528.

56 Majumdar, A.; Oden, P. I.; Carrejo, J. P.; Nagahara, L. A.; Graham, J. J.; Alexander, J. Appl. Phys. Lett. 1992, 61, 2293.

57 Xie, X. N.; Chung, H. J.; Sow, C. H.; Bettiol, A. A.; Wee, A. T. S. Adv. Mater. 2005, 17, 1386.

58 Leggett, G. J. Chem. Soc. Rev. 2006, 35, 1150.

59 Sun, S.; Leggett, G. J. Nano Lett. 2002, 2, 1223

60 Peter, M.; Li, X. M.; Huskens, J.; Reinhoudt, D. N. J. Am. Chem. Soc. 2004, 126, 11684.

61 Ahn, Y.; Ono, T.; Esashi, M. J. Mech. Sci. Technol. 2008, 22, 308.

62 Binnig, G. K.; Cherubini, G.; Despont, M.; Dürig, U. T.; Eleftheriou, E.; Pozidis H.; Vettiger, P. In: B. Bhushan, Editor, Handbook of Nanotechnology, $2^{\text {nd }}$ ed., Springer Science and Business Media Inc., Heidelberg, 2006, p. 1457-1486.

63 Lenhert, S.; Sun, P.; Wang, Y. H.; Fuchs, H.; Mirkin, C. A. Small 2007, 3, 71. 

2006, 45, 7220 .

66 Knoll, A.; Bächtold, P.; Bonan, J.; Cherubini, G.; Despont, M.; Drechsler, U.; Dürig, U.; Gotsmann, B.; Häberle, W.; Hagleitner, C.; Jubin, D.; Lantz, M. A.; Pantazi, A.; Pozidis, H.; Rothuizen, H.; Sebastian, A.; Stutz, R.; Vettiger, P.; Wiesmann, D.; Eleftheriou, E. S. Microelectron. Eng. 2006, 83, 1692.

67 Number reported by Western Digital for a WD SiliconDrive ${ }^{\circledR}$ N1x 2.5 -inch solid state drive (SSD) type hard disk.

Szoszkiewicz, R.; Okada, T.; Jones, S. C.; Li, T. D.; King, W. P.; Marder, S. R.; Riedo, E. Nano Lett. 2007, 7, 1064.

69 Duvigneau, J.; Schönherr, H.; Vancso, G. J. Langmuir 2008, 24, 10825.

70 Huck, W. T. S. Angew. Chem. Int. Ed. 2007, 46, 2754.

71 Saha, S. K.; Culpepper, M. L. Appl. Phys. Lett. 2010, 96.

72 Mamin, H. J.; Rugar, D. Appl. Phys. Lett. 1992, 61, 1003.

73 Hamann, H. F.; Martin, Y. C.; Wickramasinghe, H. K. Appl. Phys. Lett. 2004, 84, 810.

74 Huang, S. M.; Hong, M. H.; Lu, Y. F.; Lukyanchuk, B. S.; Song, W. D.; Chong, T. C. J. Appl. Phys. 2002, 91, 3268.

75 Milner, A. A.; Zhang, K. Y.; Garmider, V.; Prior, Y. Appl. Phys. A 2010, 99, 1.

76 McCarthy, B.; Zhao, Y. M.; Grover, R.; Sarid, D. Appl. Phys. Lett. 2005, 86.

77 Pylkki, R. J.; Moyer, P. J.; West, P. E. Jpn. J. Appl. Phys. Part 1 1994, 33, 3785.

78 Dinwiddie, R. B.; Pylkki, R. J.; West, P. E. Thermal conductivity contrast imaging with a scanning thermal microscope, In: Thermal conductivity 22, Wong, T. W. Editor, Technomics, Lancaster, PA, 1994, p. 668-677.

79 Gomès, S.; Trannoy, N.; Grossel, P. Meas. Sci. Technol. 1999, 10, 805.

80 Majumdar, A.; Lai, J.; Chandrachood, M.; Nakabeppu, O.; Wu, Y.; Shi, Z. Rev. Sci. Instrum. 1995, 66, 3584.

81 Fryer, D. S.; Nealey, P. F.; de Pablo, J. J. Macromolecules 2000, 33, 6439.

82 Fischer, H. Macromolecules 2005, 38, 844.

83 Hammiche, A.; Pollock, H. M.; Song, M.; Hourston, D. J. Meas. Sci. Technol. 1996, 7, 142.

84 Hammiche, A.; Bozec, L.; Conroy, M.; Pollock, H. M.; Mills, G.; Weaver, J. M. R.; Price, D. M.; Reading, M.; Hourston, D. J.; Song, M. J. Vac. Sci. Technol. B 2000, 18, 1322.

85 Rangelow, I. W.; Gotszalk, T.; Abedinov, N.; Grabiec, P.; Edinger, K. Microelectron. Eng. 2001, 57-8, 737.

86 Edinger, K.; Gotszalk, T.; Rangelow, I. W. J. Vac. Sci. Technol. B 2001, 19, 2856.

87 Nelson, B. A.; King, W. P. In: P. Avouris P.; Bhushan B.; von Klitzing K.; Sakaki, H.; Wiesendanger, R. editors. Nanoscience and Technology, Springer-Verlag, Berlin, 2006, Chapter 30.

88 Li, M. H.; Gianchandani, Y. B. J. Vac. Sci. Technol. B 2000, 18, 3600.

89 Li, M. H.; Wu, J. J.; Gianchandani, Y. B. J. Microelectromech. Syst. IEEE. 2001, 10, 3.

90 Li, M. H.; Gianchandani, Y. B. Sens. Actuators, A 2003, 104, 236. 
91

92

93

http://www.anasysinstruments.com, accessed 10-10-2010, this may disappear. http://www.veeco.com, accessed 10-10-2010, this may disappear. Mamin, H. J. Appl. Phys. Lett. 1996, 69, 433.

Chui, B. W.; Stowe, T. D.; Kenny, T. W.; Mamin, H. J.; Terris, B. D.; Rugar, D. Appl. Phys. Lett. 1996, 69, 2767.

Wright, T. L. In: Fabrication and testing of heated atomic force microscope cantilevers, Master of Science Thesis, Georgia Institute of Technology, May 2005.

King, W. P.; Kenny, T. W.; Goodson, K. E.; Cross, G.; Despont, M.; Dürig, U.; Rothuizen, H.; Binnig, G. K.; Vettiger, P. Appl. Phys. Lett. 2001, 78, 1300.

Lee, J.; Beechem, T.; Wright, T. L.; Nelson, B. A.; Graham, S.; King, W. P. J Microelectromech. Syst. IEEE 2006, 15, 1644.

Ravi, T. S.; Marcus, R. B.; Liu, D. J. Vac. Sci. Technol. B 1991, 9, 2733.

Dai, Z.; King, W. P.; Park, K. Nanotechnology 2009, 20.

Dai, Z. T.; Corbin, E. A.; King, W. P. Nanotechnology 2010, 21.

Lee, D. W.; Oh, I. K. Microelectron. Eng. 2007, 84, 1041.

Chiou, C. H.; Chang, S. J.; Lee, G. B.; Lee, H. H. Jpn. J. Appl. Phys. Part 1 2006, 45, 208.

Yang, Z. X.; Yu, Y.; Li, X. X.; Bao, H. F. Microelectron. Reliab. 2006, 46, 805.

Lee, C. S. Y.; Kim, S. G.; Jeong, S. Y.; Ahn, S. H.; Jin, W. H.; Jang, S. S.; Cho, I. J.; Kim, Y.

S.; Nam, H. J. Sens. Actuators, A 2007, 139, 12.

Kölzer, J.; Oesterschulze, E.; Deboy, G. Microelectron. Eng. 1996, 31, 251.

Fletcher, D. A.; Kino, G. S.; Goodson, K. E. Microscale Therm. Eng. 2003, 7, 267.

Sarua, A.; Ji, H. F.; Kuball, M.; Uren, M. J.; Martin, T.; Hilton, K. P.; Balmer, R. S. Electron Dev. T. IEEE 2006, 53, 2438.

Ju, Y. S.; Goodson, K. E. J. Heat Trans. T. ASME 1998, 120, 306.

Grauby, S.; Dilhaire, S.; Jorez, S.; Claeys, W. Electron Dev. Lett. IEEE 2005, 26, 78.

Nelson, B. A.; King, W. P. Sens. Actuators, A 2007, 140, 51.

Chui, B. W.; Asheghi, M.; Ju, Y. S.; Goodson, K. E.; Kenny, T. W.; Mamin, H. J. Microscale Therm. Eng. 1999, 3, 217.

Abel, M. R.; Wright, T. L.; King, W. P.; Graham, S. Compon. Pack. T. IEEE 2007, 30, 200.

Serrano, J. R.; Phinney, L. M.; Kearney, S. P. J. Micromech. Microeng. 2006, 16, 1128.

We note that polymers typically have a temperature range for the thermal transition points (i.e. $T_{m}$ and $T_{g}$ ) compared to the sharp defined melting points for small organic molecules.

Gedde, U. W. Polymer Physics, Kluwer Academic Publishers, Dordrecht, 1999.

Mandelkern, L.; Alamo, R. G. In: Mark, J. E., editor, Physical Properties of Polymers Handbook, $2^{\text {nd }}$ ed., Springer Science and Business Media LCC, New York, 2007, p. 168.

Plazek, D. J.; Ngai, K. L. In: Mark, J. E., editor, Physical Properties of Polymers Handbook, $2^{\text {nd }}$ ed., Springer Science and Business Media LCC, New York, 2007, p. 197.

The major part of the temperature ramp can be operated in force feedback mode. Hence, the thermal expansion of the polymer film is compensated for by the Z-piezo, maintaining the preset cantilever deflection (i.e. constant force). The feedback is switched off at $\sim 30{ }^{\circ} \mathrm{C}$ before the expected softening transition. With this method the increase in cantilever deflection is significantly reduced.

Nairn, J. A.; Zoller, P. J. Mater. Sci. 1985, 20, 355.

Tao, Z.; Bhushan, B. Tribol. Lett. 2006, 21, 1.

King, W. P. dissertation, Stanford University, 2002. 
Nelson, B. A.; King, W. P. Rev. Sci. Instrum. 2007, 78, 023702.

Lantz, M. A.; Gotsmann, B.; Dürig, U. T.; Vettiger, P.; Nakayama, Y.; Shimizu, T.; Tokumoto, H. Appl. Phys. Lett. 2003, 83, 1266.

Wong, E. W.; Sheehan, P. E.; Lieber, C. M. Science 1997, 277, 1971.

Larsen, T.; Moloni, K.; Flack, F.; Eriksson, M. A.; Lagally, M. G.; Black, C. T. Appl. Phys. Lett. 2002, 80, 1996.

Berber, S.; Kwon, Y. K.; Tomanek, D. Phys. Rev. Lett. 2000, 84, 4613.

Kim, P.; Shi, L.; Majumdar, A.; McEuen, P. L. Phys. Rev. Lett. 2001, 8721, 215502.

Fletcher, P. C.; Felts, J. R.; Dai, Z. T.; Jacobs, T. D.; Zeng, H. J.; Lee, W.; Sheehan, P. E.; Carlisle, J. A.; Carpick, R. W.; King, W. P. ACS Nano 2010, 4, 3338.

Kappes, R. S.; Li, C.; Butt, H. J.; Gutmann, J. S. New J. Phys. 2010, 12, 083011.

Kim, K. J.; King, W. P. Appl. Therm. Eng. 2009, 29, 1631.

Nelson, B. A.; King, W. P. Nanosc. Microsc. Therm. Eng. 2008, 12, 98.

Gotsmann, B.; Lantz, M. A.; Dürig, U. Nanoscale Thermal and Mechanical Interactions Studies using Heatable Probes, In: Nanotechnology, Fuchs, H. Editor, Wiley-VCH Verlag GmbH \& Co. KGaA, Weinheim, 1994, p. 121-169.

Rohsenow, W. M.; Hartnett, J. P.; Ganić, E. N. Handbook of Heat Transfer Fundamentals, $2^{\text {nd }}$ ed., McGraw-Hill Book Company, New York, 1985.

Hu, X. J.; Jain, A.; Goodson, K. E. Int. J. Therm. Sci. 2008, 47, 820.

Gebhart, B.; Jaluria, Y.; Mahajan, R. L.; Sammakia, B. Buoyancy-Induced Flows and Transport, Hemisphere, New York, 1988.

Zhou, J.; Berry, B.; Douglas, J. F.; Karim, A.; Snyder, C. R.; Soles, C. Nanotechnology 2008, 19.

Meyers, G.; Pastzor, A.; Kjoller, K. Am. Lab. 2007, 39, 9.

King, W. P.; Saxena, S.; Nelson, B. A.; Weeks, B. L.; Pitchimani, R. Nano Lett. 2006, 6, 2145.

Lee, J.; King, W. P. Rev. Sci. Instrum. 2007, 78, 126102.

Bullen, D.; Wang, X. F.; Zou, J.; Chung, S. W.; Mirkin, C. A.; Liu, C. J. Microelectromech. S. 2004, 13, 594.

Pedrak, R.; Ivanov, T.; Ivanova, K.; Gotszalk, T.; Abedinov, N.; Rangelow, I. W.; Edinger, K.; Tomerov, E.; Schenkel, T.; Hudek, P. J. Vac. Sci. Technol. B 2003, 21, 3102.

Privorotskaya, N. L.; King, W. P. Microsyst. Technol. 2009, 15, 333.

Lee, J.; King, W. P. Rev. Sci. Instrum. 2008, 79, 054901.

Guo, F. A.; Trannoy, N.; Lu, J. Mater. Chem. Phys. 2006, 96, 59.

Häberle, W.; Pantea, M.; Hoerber, J. K. H. Ultramicroscopy 2006, 106, 678.

King, W. P. J. Micromech. Microeng. 2005, 15, 2441.

Park, K.; Lee, J.; Zhang, Z. M.; King, W. P. Rev. Sci. Instrum. 2007, 78, 043709.

Vettiger, P.; Cross, G.; Despont, M.; Drechsler, U.; Dürig, U.; Gotsmann, B.; Häberle, W.; Lantz, M. A.; Rothuizen, H. E.; Stutz, R.; Binnig, G. K. Nanotechnol. T. IEEE 2002, 1, 39.

Sheehan, P. E.; Whitman, L. J.; King, W. P.; Nelson, B. A. Appl. Phys. Lett. 2004, 85, 1589.

Salaita, K.; Wang, Y. H.; Mirkin, C. A. Nat. Nanotechnol. 2007, 2, 145.

Nelson, B. A.; King, W. P.; Laracuente, A. R.; Sheehan, P. E.; Whitman, L. J. Appl. Phys. Lett. 2006, 88, 033104.

Lee, W. K.; Whitman, L. J.; Lee, J.; King, W. P.; Sheehan, P. E. Soft Matter 2008, 4, 1844. 
153 Hua, Y. M.; Saxena, S.; Henderson, C. L.; King, W. P. J. Micro-Nanolith. Mem. 2007, 6, 023012 .

154 Hua, Y. M.; King, W. P.; Henderson, C. L. Microelectron. Eng. 2008, 85, 934.

155 Knoll, A. W.; Pires, D.; Coulembier, O.; Dubois, P.; Hedrick, J. L.; Frommer, J.; Dürig, U. $A d v$. Mater. 2010, 22, 3361.

156 Pires, D.; Hedrick, J. L.; De Silva, A.; Frommer, J.; Gotsmann, B.; Wolf, H.; Despont, M.; Dürig, U.; Knoll, A. W. Science 2010, 328, 732.

157 Coulembier, O.; Knoll, A.; Pires, D.; Gotsmann, B.; Dürig, U.; Frommer, J.; Miller, R. D.; Dubois, P.; Hedrick, J. L. Macromolecules 2010, 43, 572.

158 Basu, A. S.; McNamara, S.; Gianchandani, Y. B. J. Vac. Sci. Technol. B 2004, 22, 3217.

159 Fenwick, O.; Bozec, L.; Credgington, D.; Hammiche, A.; Lazzerini, G. M.; Silberberg, Y. R.; Cacialli, F. Nat. Nanotechnol. 2009, 4, 664.

160 Wang, D. B.; Kim, S.; Underwood, W. D.; Giordano, A. J.; Henderson, C. L.; Dai, Z. T.; King, W. P.; Marder, S. R.; Riedo, E. Appl. Phys. Lett. 2009, 95, 203188.

161 Wang, D. B.; Szoszkiewicz, R.; Lucas, M.; Riedo, E.; Okada, T.; Jones, S. C.; Marder, S. R.; Lee, J.; King, W. P. Appl. Phys. Lett. 2007, 91, 243104.

162 Wang, D. B.; Kodali, V. K.; Underwood, W. D.; Jarvholm, J. E.; Okada, T.; Jones, S. C.; Rumi, M.; Dai, Z. T.; King, W. P.; Marder, S. R.; Curtis, J. E.; Riedo, E. Adv. Funct. Mater. 2009, 19, 3696. 


\section{Chapter 3}

\section{Nanoscale Thermal AFM of Polymers: Transient Heat Flow Effects}

This Chapter describes the thermal transport around the nanoscale contact area between a heated atomic force microscopy (AFM) probe tip and the specimen under investigation, which is a central issue in scanning thermal microscopy (SThM). Polarized light microscopy and AFM imaging of the temperature-induced crystallization of poly(ethylene terephthalate) (PET) films in the region near the tip were used in this study to unveil the lateral heat transport. The radius of the observed lateral surface isotherm at $133{ }^{\circ} \mathrm{C}$ ranged from $2.2 \pm 0.5 \mu \mathrm{m}$ up to $18.7 \pm 0.5 \mu \mathrm{m}$ for tip-polymer interface temperatures between $200{ }^{\circ} \mathrm{C}$ and $300{ }^{\circ} \mathrm{C}$ with contact times varying from 20 seconds up to 120 seconds, respectively. In addition, the heat transport into polymer films was assessed by measurements of the thermal expansion of poly(dimethyl siloxane) (PDMS) films with variable thickness on silicon supports. Our data showed that heat transport in the specimen normal $(z)$ direction occurred to depths exceeding $1000 \mu \mathrm{m}$ using representative non steady state SThM conditions (i.e. heating from $40{ }^{\circ} \mathrm{C}$ to $180{ }^{\circ} \mathrm{C}$ at a rate of $10{ }^{\circ} \mathrm{C} \mathrm{s}^{-1}$ ). Based on the experimental results, a 1D steady state model for heat transport was developed, which shows the temperature profile close to the tip-polymer contact. The model also indicates that $\leq 1 \%$ of the total power generated in the heater area, which is embedded in the cantilever end, is transported into the polymer through the tip-polymer contact interface. Our results complement recent efforts in the evaluation and improvement of existing theoretical models for thermal AFM, and facilitate further developments of SThM for nanoscale thermal materials characterization and/or manipulation via scanning thermal lithography (SThL).

\footnotetext{
* Parts of this chapter have been published in: Duvigneau, J.; Schönherr, H.; Vancso, G. J. ACS Nano 2010, 4, 6932 .
} 


\subsection{Introduction}

Nanoscale thermal analysis (NanoTA) is an emerging technique in nanotechnology for the determination of surface transition temperatures of materials with high spatial resolution, complementing well established bulk methods, such as differential scanning calorimetry (DSC), thermomechanical analysis (TMA) and dynamic mechanical analysis (DMA). ${ }^{1-5}$ In addition, NanoTA has found applications in tip-based nanofabrication. ${ }^{6-8}$

NanoTA relies on the use of heatable atomic force microscopy (AFM) probes that possess typical tip radii of curvature of 30 nanometer or less and are equipped with a resistive Joule heater, which is embedded at the cantilever end. ${ }^{9,10}$ As an expansion of earlier scanning thermal microscopy, NanoTA promises to yield access to surface transition temperature characteristics of materials at a spatial resolution of 100 nanometers or better (see Chapter 2). Originally these heatable AFM probes were introduced for the development of high data density data storage devices. ${ }^{11-13}$ These devices, which function via nanoscale thermomechanical material manipulation, have been very recently expanded to create $2 \mathrm{D}$ and even $3 \mathrm{D}$ lithographic structures. ${ }^{14,15}$ In their recent papers, Knoll and coworkers have reported on the 3D patterning in polymer resist layers using scanning thermal lithography (SThL). ${ }^{14,15}$ Their 3D reproduction of a world map in a poly(phthalaldehyde) film also demonstrated the possible high throughput of this patterning technique with $\sim 40 \mathrm{~nm}$ resolution or better. In addition to thermomechanical material manipulation, SThL has proven its feasibility in localized thermochemical surface functionalization and subsequent derivatization for the development of e.g. (bio)sensors. ${ }^{16-20}$

For the accurate and precise thermal characterization and manipulation of materials on the nanoscale, the long and short range temperature gradients that arise from the heated cantilever and extend into its surrounding must be understood. To date most work that addressed these thermal gradients relied on the use of (over)simplified theoretical models and simulations without complementing direct experimental support. Due to the overall complex geometry of the cantilever heat source, which is positioned relatively far away from the surface, the resulting thermal transport covers different regimes. ${ }^{21,22}$

Figure 3.1 shows a front view of the cantilever end of a heated thermal probe in contact with a polymer film on a solid substrate. The integrated heater area is maintained at a pre-set temperature of $T_{H}$ set by applying a voltage over the cantilever legs. According to calculations of King and coworkers ${ }^{1}$ approximately $20 \%$ of the total power generated at the heater area (on the order of a few $\mathrm{mW}$ ) is lost to the surrounding atmosphere above the cantilever $\left(q_{\text {ambient }}\right)$. A large fraction of the generated power is dissipated via the air gap $\left(d_{\text {gap }} \sim 4 \mu \mathrm{m}\right)$ between cantilever legs and 
polymer film $\left(q_{g a p}\right)$. The calculations show that only about $0.1 \%$ of the generated power is conducted through the probe-polymer contact $\left(q_{i}\right)$. Radiative heat transport was concluded to be insignificant. ${ }^{23,24}$ These different pathways of heat conduction contribute to a different extent and at different length scales to the heating of the substrate. Calculations by Hoerber and coworkers ${ }^{25}$ showed for example that substrates with good heat conductivity are substantially less heated as compared to poor thermal conductors, when they are brought into contact with the heated probe. The surface temperature increase for a glass $\left(\lambda \sim 1 \mathrm{~W} \mathrm{~m}^{-1} \mathrm{~K}^{-1}\right)$ and a silicon $\left(\lambda \sim 350 \mathrm{~W} \mathrm{~m}^{-1} \mathrm{~K}^{-1}\right)$ substrate below a cantilever heated to $440{ }^{\circ} \mathrm{C}\left(d_{\text {gap }}=0.5 \mu \mathrm{m}\right)$ was calculated to be $100{ }^{\circ} \mathrm{C}$ and $20^{\circ} \mathrm{C}$, respectively.

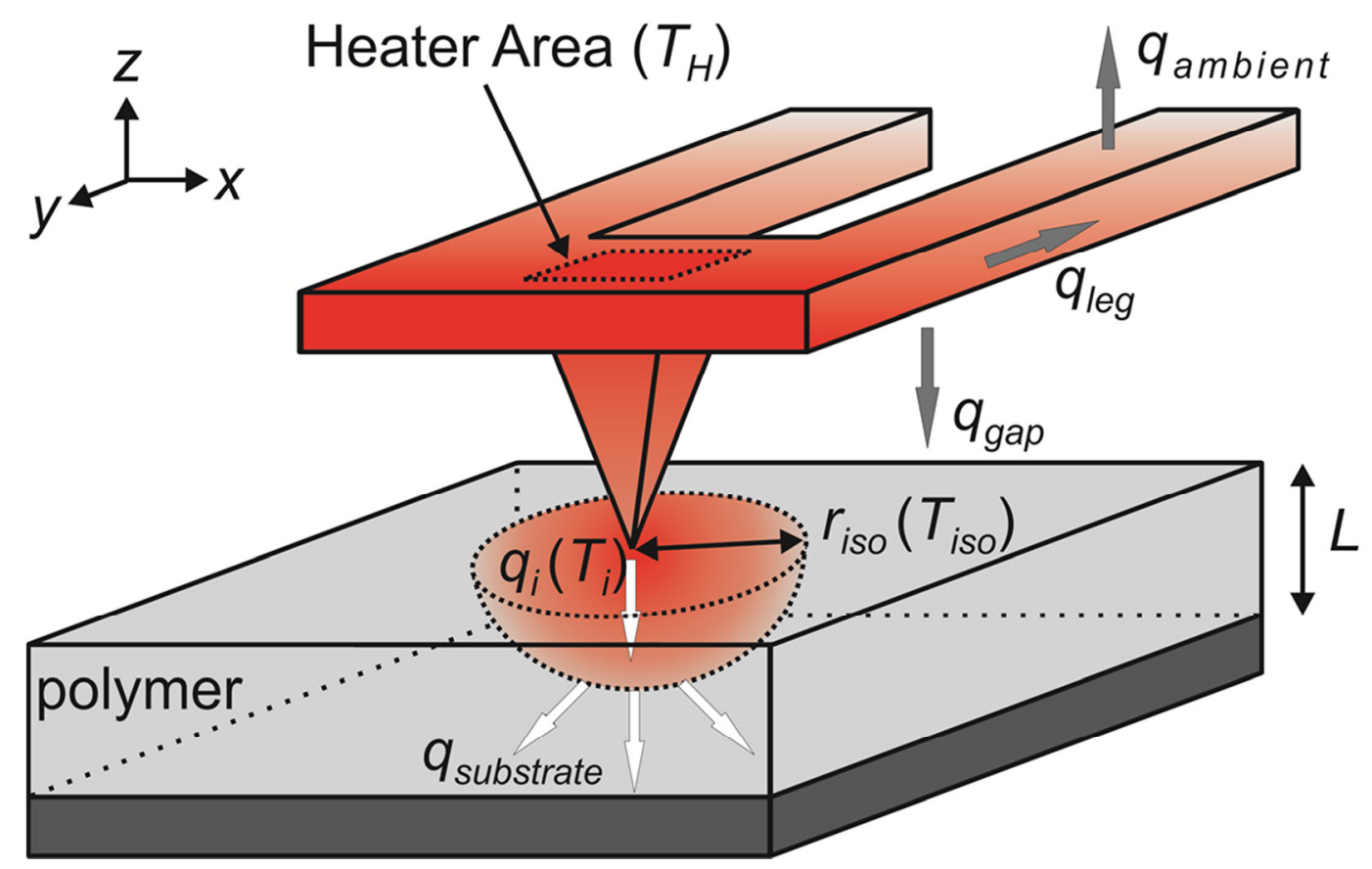

Figure 3.1: Schematic front view of a batch fabricated heatable AFM probe in contact with a polymer film on a solid substrate. The integrated heater area, positioned above the tip is brought to a pre-set temperature $T_{H}$, which is controlled by applying a voltage over the cantilever legs. The resulting heat fluxes (q) are represented by the white and grey arrows, indicating the different pathways of heat loss from the heater area.

Furthermore Hoerber et al. showed that the temperature at the probe-substrate interface $\left(T_{i}\right)$ strongly depends on the thermal conductivity of the surface under investigation. For a tip radius of $10 \mathrm{~nm}$ and a cantilever heated to $440{ }^{\circ} \mathrm{C}$ their model predicted a tip-interface temperature of $\sim 320^{\circ} \mathrm{C}$ for glass and $\sim 100{ }^{\circ} \mathrm{C}$ for silicon surfaces, respectively. Nelson and King confirmed these trends in calculations that show a correlation of $T_{i}$ with the ratio of substrate to tip thermal conductivity. ${ }^{21}$ In agreement with the results by Hoerber et al., these results revealed that a significantly shallower thermal gradient exists within the tip for low conductivity materials 
compared to high conductivity materials (see Chapter 2). King and coworkers performed another interesting finite element simulation of a cantilever positioned in air. ${ }^{26}$ During heating with a power of $8 \mathrm{~mW}$ for $100 \mu \mathrm{s}$ the temperature gradient in the direction of the principal axis of the cantilever covered already $>120 \mu \mathrm{m}$. The air close to the heater area reached temperatures $>400{ }^{\circ} \mathrm{C}^{26}$ In addition, these simulations convincingly illustrated the anticipated cantilever shape effect on the heat diffusion from the cantilever to its surroundings.

While abundant simulation data can be found in the literature, experimental confirmation remains scarce. King and coworkers ${ }^{27}$ estimated that about $75 \%$ of the power generated in the probe reaches the substrate directly below the heated cantilever using $\mathrm{a} \sim 140 \mathrm{~nm}$ wide platinum resistance thermometer fabricated on a $\mathrm{SiO}_{2}$ surface. These data are very close to the simulation results mentioned above. These measurements, however, did not provide insights in the lateral evolution of thermal transport, most likely due to the strong dependency of the nano-thermometer response as a function of the cantilever orientation with respect to the thermometer position. ${ }^{27}$ In addition, the experimental results are limited to a relatively good thermal conductor $\left(\mathrm{SiO}_{2}\right)$, whereas in many applications polymers (which are poor thermal conductors) and especially polymer thin films are applied. For this important class of materials a substantial increase in surface temperature below the heated cantilevers is expected. Hence this problem was studied in detail in this chapter.

The results shown in this chapter provide a deepened insight in the range of the temperature gradient in SThM $(i)$ in the lateral direction close to the tip-sample contact $(<10 \mu \mathrm{m})$ and $(\mathrm{ii})$ in the vertical direction far away from the tip-sample contact into polymer films $(z>>100 \mu \mathrm{m})$ during non steady state heating conditions. The results obtained are of interest for the evaluation and improvement of existing theoretical models for SThM, as well as for further developments of SThM-based approaches aiming at high resolution thermal material characterization and/or manipulation. 


\subsection{Results and discussion}

We assessed the temperature gradients in SThM using two polymer systems, i.e. amorphous PET films and crosslinked PDMS elastomer films with varying thickness values. PET quenched from the melt to an amorphous state was chosen as crystallization will proceed radially upon heating with a thermal AFM probe to temperatures $>133{ }^{\circ} \mathrm{C}$. Crosslinked PDMS elastomer was chosen as it exhibits a high thermal expansion coefficient $\left(\alpha \sim 200 \times 10^{-6}{ }^{\circ} \mathrm{C}^{-1}\right)^{28}$ and good thermal stability.

The corresponding thermal gradients were estimated from an analysis of the lateral dimensions of crystallites obtained in the heat-induced crystallization of PET (Figure 3.2) and the variation in the thermal expansion of PDMS films as a function of film thicknesses on silicon (Figure 3.3), respectively.

A

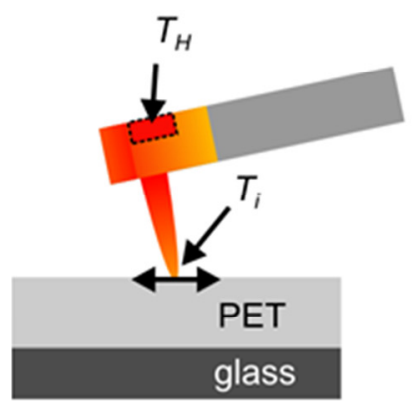

B

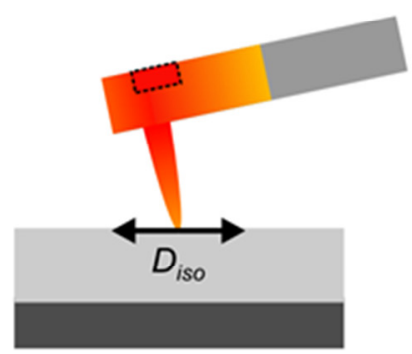

C

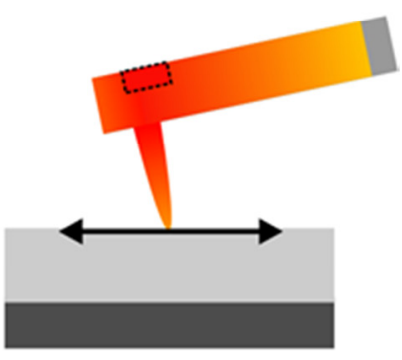

increasing time / temperature

$A^{\prime}$

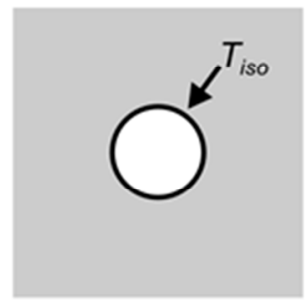

$B^{\prime}$

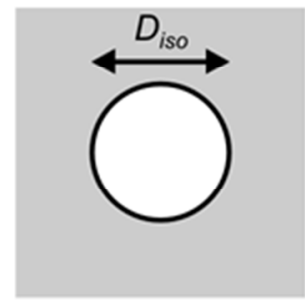

C'

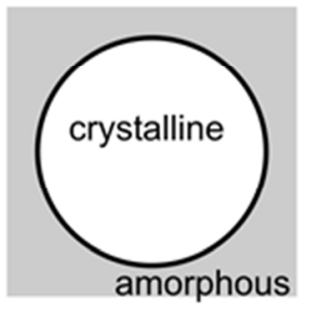

Figure 3.2: Schematic cross-sectional side view of a heated AFM probe in contact with an amorphous PET film prepared by rapidly cooling PET from the melt to room temperature (the cantilever is mounted at an inclination angle of $\sim 12^{\circ}$ ). The thermal gradient, marked by the double-headed arrows on the PET surface, underneath the heated AFM probe induces localized crystallization as long as the temperature remains above the threshold temperature for crystallization of PET $\left(\sim 133^{\circ} \mathrm{C}\right)$. The resulting size of the semi-crystalline domains $\left(D_{\text {iso }}=2 r_{\text {iso }}\right)$ depends on the holding time and the probe temperature $\left(T_{i}\right)$ (A-C). Bottom: schematic top view images of the corresponding semi-crystalline domains $\left(A^{\prime}-C^{\prime}\right)$. The outer semi-crystalline domain boundary can be considered as an isotherm of temperature $T_{\text {iso }}\left(\sim 133^{\circ} \mathrm{C}\right)$. 
A

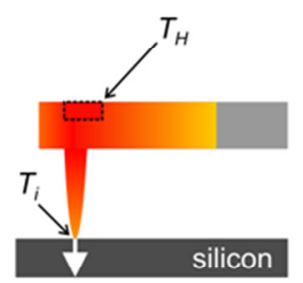

$\overbrace{}^{A^{\prime}} \Delta L_{\text {tot }}=\Delta L_{S i}$

$T\left[{ }^{\circ} \mathrm{C}\right]$
B
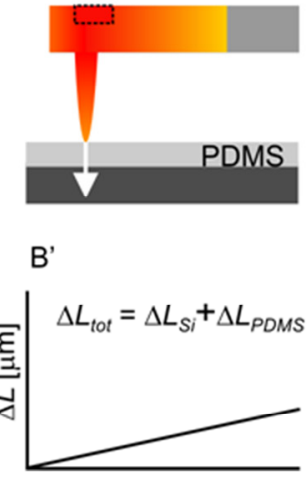

$T\left[{ }^{\circ} \mathrm{C}\right]$
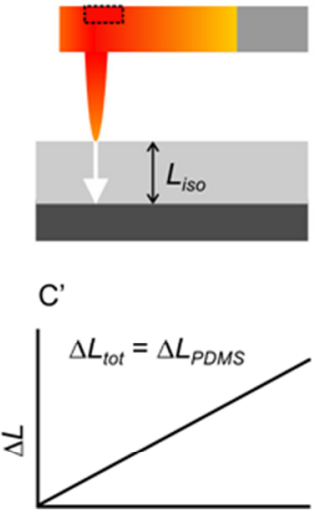

$T\left[{ }^{\circ} \mathrm{C}\right]$
D
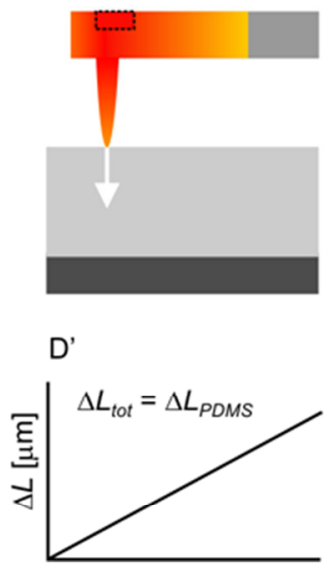

$T\left[{ }^{\circ} \mathrm{C}\right]$

Figure 3.3: Schematic cross-sectional side view of a heated AFM probe in contact with a silicon substrate $(A)$ or PDMS films with increasing thicknesses on silicon substrates (B-D) (the cantilever is mounted at an inclination angle of $\sim 0^{\circ}$ ). The non steady state heat penetration depth directly below the tip-sample contact at time $t$ and temperature $T(t)$ during temperature scans is indicated by the white arrow (z direction). Graphs A' to D' show the respective schematic thermal expansion $(\Delta L)$ vs. temperature plots. Under conditions shown in $C$, the heat flux is not sufficient to raise the temperature at the silicon PDMS interface and as a consequence the thermal expansion for even thicker films as a function of temperature becomes independent of the PDMS film thickness (D).

\subsubsection{Heat induced localized PET crystallization}

To analyze the lateral heat transport, heated AFM probes were brought into contact with an amorphous PET film. Here they induce the formation of spherulitelike crystallites (Figure 3.4), when the substrate temperature reaches temperatures above the threshold temperature for crystallization $\left(T_{c}\right)$. The evaluation of the size of the semi-crystalline domains formed (diameter $D_{i s o}=2 r_{i s o}$ ) as a function of $T_{i}$ and heating time provides insight in the lateral evolution of $T_{c}$ in the form of an isotherm ( $T_{\text {iso }}$ ) (Figure 3.5, compare also Figure 3.1). For a comparison $T_{\text {iso }}$ was determined as $133{ }^{\circ} \mathrm{C}$ from the onset of crystallization of amorphous PET samples observed in DSC heating traces (data not shown). Figure 3.5 shows AFM contact mode deflection images acquired after heated AFM probe induced growth of semi-crystalline PET domains at $T_{i}=250{ }^{\circ} \mathrm{C}$ for different holding times. The semi-crystalline nature of the domains was confirmed with polarized light microscopy (see Figure 3.4) and local NanoTA melting point measurements. The observed birefringence in polarized light microscopy revealed the nucleation and growth of many small crystallites, which possess negative birefringence and resemble in some areas crude spherulites. From the NanoTA experiments a typical melting transition of $188 \pm 3{ }^{\circ} \mathrm{C}$ was determined, independent of the radial position within the semi-crystalline domain. This marked 
deviation from the bulk melting point $\left(T_{m}(\mathrm{DSC})=235^{\circ} \mathrm{C} \pm 5{ }^{\circ} \mathrm{C}\right)$ is attributed to i) the short crystallization times in a non-uniform temperature field, which results in the formation of imperfect semi-crystalline domains, as well as to $i i)$ the known complex melting behavior of PET. ${ }^{29,30}$

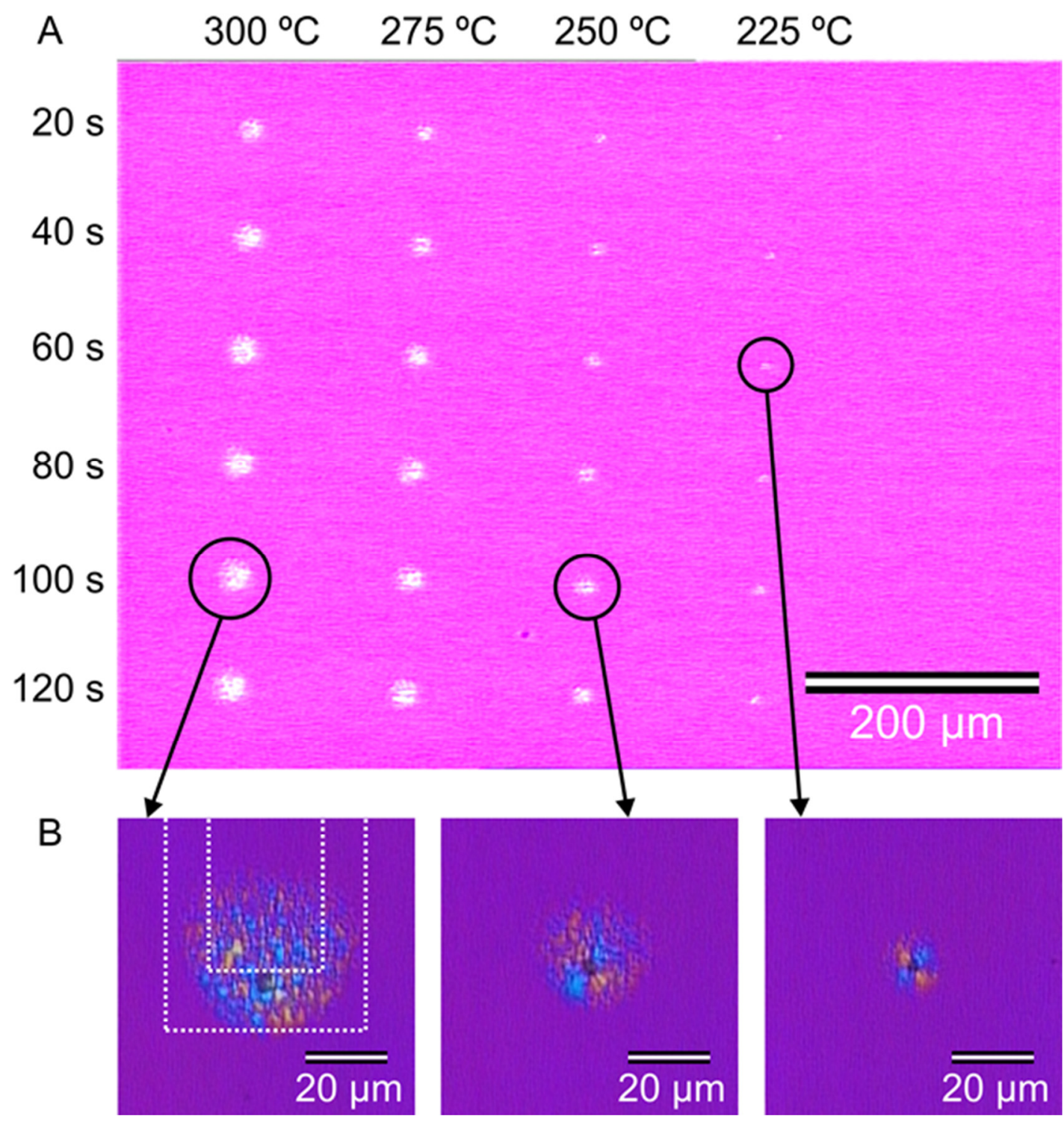

Figure 3.4: Polarized light microscopy images (using crossed polars and a lambda plate) of semi-crystalline PET domains formed in the surface region near the heated AFM probe tip. A) Low magnification overview image of part of the formed domains. The tip temperatures and heating times are denoted at the top and left side of the image, respectively. Note: In A the color balance was adjusted to enhance the contrast between the amorphous PET and semi-crystalline PET domains. B) Higher magnification images of the corresponding crystals within the black circles in $A$ (turned $90^{\circ}$ clock wise with respect to $A$ ). The observed birefringence is a result of the semi-crystalline morphology within these domains. The cantilever orientation is schematically shown in B (left image, dotted lines, not to scale). Note: Localized crystallization was also observed in experiments in which the heated cantilever was positioned $1 \mu \mathrm{m}$ above the PET film. 

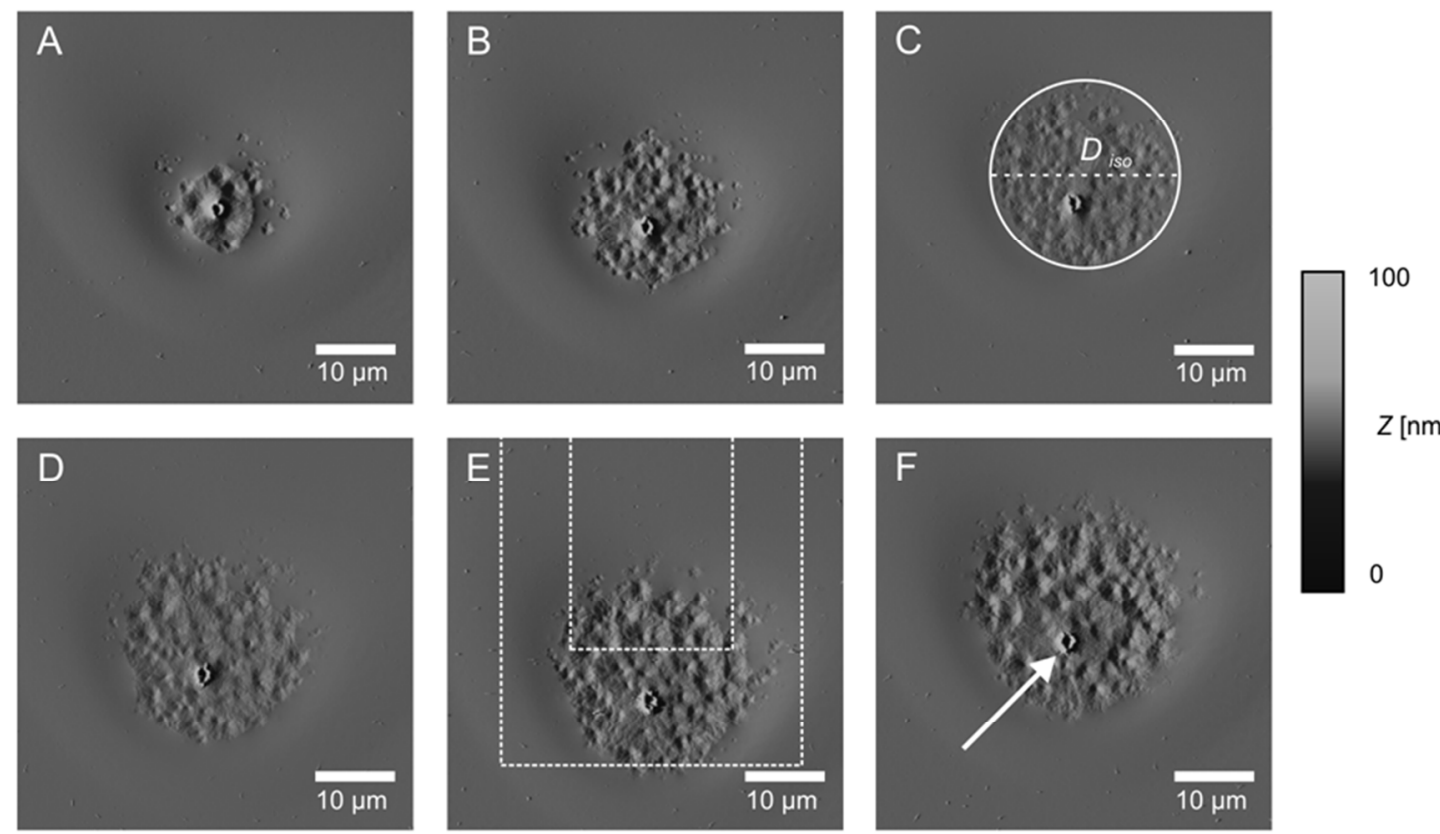

Figure 3.5: AFM contact mode deflection images of semi-crystalline domains of PET grown by localized crystallization induced by the heated probe tip. A nominal tip temperature of $250{ }^{\circ} \mathrm{C}$ was kept constant for 20 seconds (A), 40 seconds (B), 60 seconds (C), 80 seconds (D), 100 seconds $(E)$ and 120 seconds (F), respectively. $D_{\text {iso }}$ was measured as the diameter of a circle fitted to the contour of the semi-crystalline domain as depicted in (C). The cantilever orientation is schematically shown in $E$ (dotted lines, not to scale). The white arrow in $F$ points to the residual indent at the tip-PET contact point.

For the determination of $D_{\text {iso }}$ the semi-crystalline domains were assumed to be circular in shape. Most of the deviations from the assumed circularity were observed at that side of the semi-crystalline domains (see Figure 3.5) that was closest to the cantilever base for practically the entire range of holding times and tip temperatures used. This deviation is attributed to the fact that the cantilever is mounted at an inclination angle of $12^{\circ}$ with respect to the cantilever holder plane. Since about $75 \%$ of the heat generated in the cantilever legs reaches into the underlying polymer film according to simulations by King and coworkers, ${ }^{27}$ the increased separation distance at few micrometers distance away from the tip-polymer contact point is sufficient to cause a substantial decrease in surface temperature. In addition, the tip-polymer contact point was typically not in the centre of the domains formed, but closer to the cantilever end. The non-circular shapes of the domains are therefore in agreement with the notion that most of the heat is conducted into the cantilever legs, from where it is dissipated over the air gap between the cantilever and polymer film. 
Figure 3.6 shows a plot of $D_{\text {iso }}$ as a function of time for pre-set temperatures between $200{ }^{\circ} \mathrm{C}$ and $300{ }^{\circ} \mathrm{C}$. The observed values of $D_{\text {iso }}$ range from $\sim 2.4 \pm 1.0 \mu \mathrm{m}$ up to $\sim 37.4 \pm 1.0 \mu \mathrm{m}$ for 20 seconds at $200{ }^{\circ} \mathrm{C}$ and $120 \mathrm{~s}$ at $300{ }^{\circ} \mathrm{C}$, respectively. Following the initial increase in rate of propagation of the lateral isothermal boundary, the observed rate reaches a plateau value $\left(D_{\max }\right)$. The obtained values of $D_{\max }$ are plotted versus probe temperature in Figure 3.7.

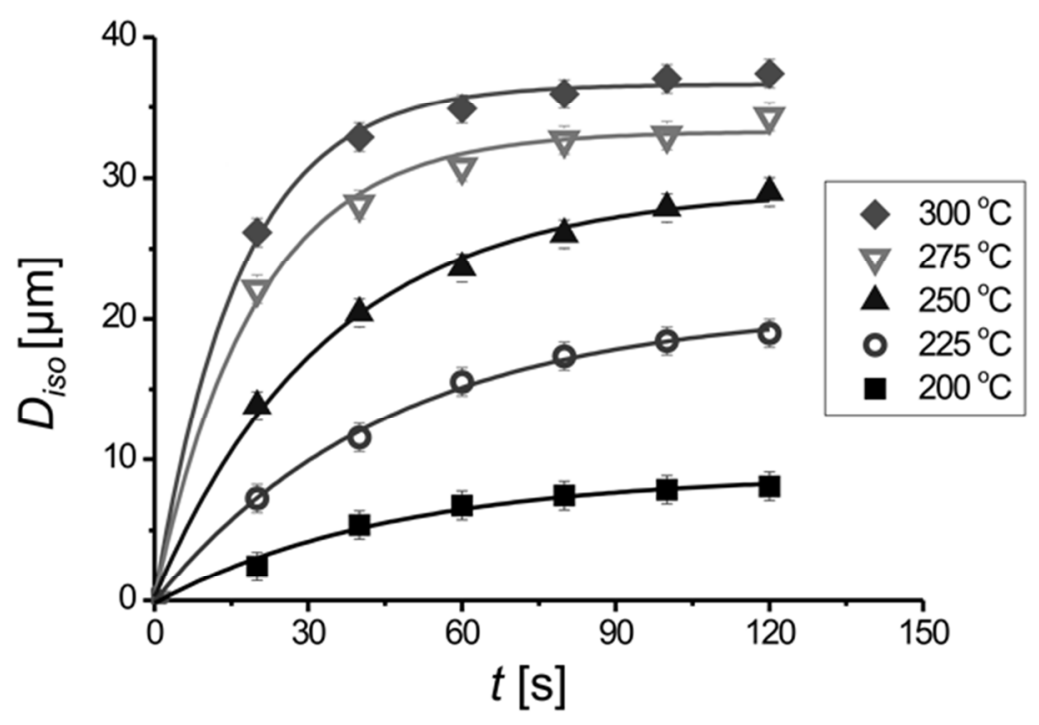

Figure 3.6: $D_{\text {iso }}$ as a function of time for heated AFM probe tip induced growth of semicrystalline PET domains with pre-set probe temperatures $\left(T_{i}\right)$ in the range of $200{ }^{\circ} \mathrm{C}$ to 300 ${ }^{\circ} \mathrm{C}$. The solid lines are exponential fits to the data and serve as a guide for the eye.

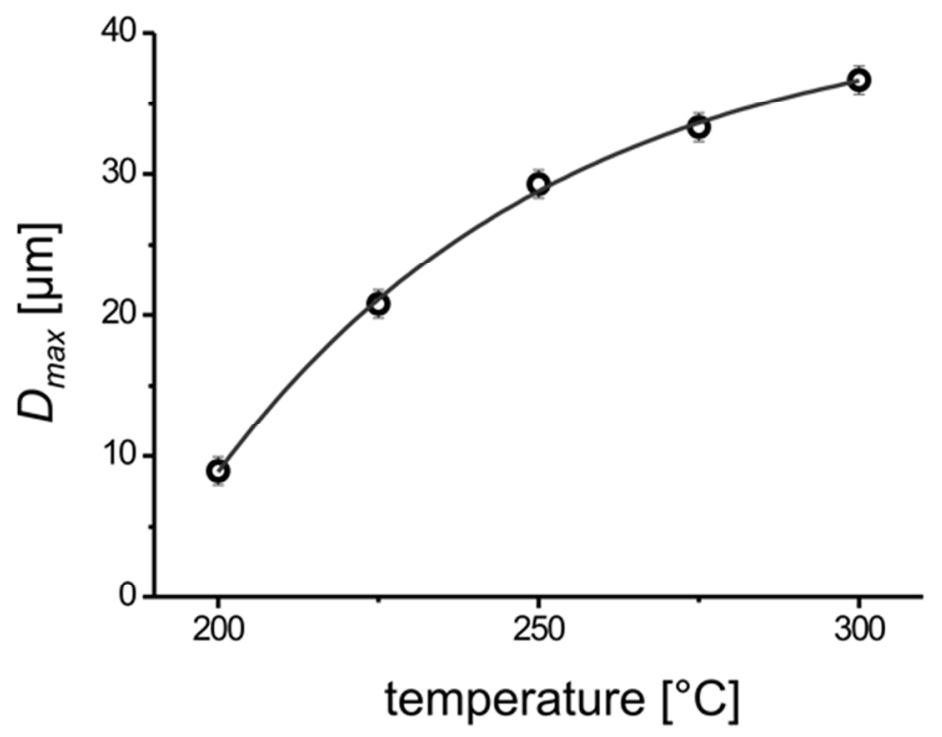

Figure 3.7: Maximum PET domain size $\left(D_{\max }\right)$ as a function of the probe temperature. The solid line is an exponential fits to the data and serves as a guide for the eye. 


\subsubsection{PDMS film thickness dependent thermal expansion}

To analyze the heat transport into polymer films in the $z$ direction under a heated NanoTA probe, the film thickness dependent thermal expansion of PDMS films on silicon substrates was investigated (Figure 3.3). During conventional NanoTA measurements the deflection signal is monitored as a function of temperature after bringing the probe into contact with the polymer film. The force feedback is switched off during such an experiment. When the temperature is ramped up, the polymer film thermally expands. This results in an increase of the deflection signal (i.e. the cantilever bends up). Once a transition temperature (e.g. $T_{g}$ or $T_{m}$ ) is reached, a pronounced drop in the deflection signal is observed. The onset of the transition is assigned to the temperature at which the slope of the deflection vs. temperature plot becomes zero. The upward bending of the cantilever significantly increases the load (normal force) applied by the cantilever on the film before the transition temperature is reached. The increase, which may amount to several $100 \mathrm{nN}$ (a factor of 30), increases the tip-polymer contact area and hence affects the thermal transport through the probe. To avoid this increase in forces, the AFM was operated in a force feedback mode in our experiments maintaining a constant force of $10 \mathrm{nN}$. To detect the thermal expansion of the polymer film, the Z-sensor position of the closed loop feedback system was monitored, which provides a direct measure of the thermal expansion $(\Delta L)$ of the film (Figure 3.3A'-D').

It is well known that the linear thermal expansion $(\Delta L)$ of material A (e.g. PDMS) with thickness $L_{A}$ on top of material B (e.g. silicon) with thickness $L_{B}$ with a total thickness of $L$ in a uniform temperature field $(\Delta T)$ can be expressed as the combined thermal expansion of both materials. ${ }^{31-33}$ Despite the fact that the temperature field in its surrounding is non uniform and as such becomes a function of time $(t)$ and position $(z)$, the observed thermal expansion at time $t_{1}$ is the combined thermal expansion of both the material under investigation and the underlying material. This scenario only holds if the heat transport is sufficient to induce an increase in temperature in material B. When this requirement is no longer met, only material A contributes to the observed thermal expansion and hence $\Delta L$ becomes independent of $L_{A}$ (compare Figure 3.3, in which PDMS corresponds to material A).

Thus, by measuring the film thickness dependence of the thermal expansion of elastomeric PDMS films (with $\alpha \sim 200 \times 10^{-6}{ }^{\circ} \mathrm{C}^{-1}$ ) ${ }^{28}$ on silicon substrates (with $\left.\alpha \sim 3 \times 10^{-6}{ }^{\circ} \mathrm{C}^{-1}\right)^{34}$ by NanoTA measurements, insight in the length scale of heat transport in the $z$ direction is obtained during non steady state conditions typically used in NanoTA. The over 60 fold higher thermal expansion coefficient of PDMS compared to silicon provides a good resolution for determining $T_{i s o}$. 
Figure 3.8A shows the measured thermal expansion as a function of temperature for temperature ramps between $40{ }^{\circ} \mathrm{C}$ and $180{ }^{\circ} \mathrm{C}$ at $10{ }^{\circ} \mathrm{C} \mathrm{s}^{-1}$ on PDMS films on silicon with film thicknesses ranging from 0 to $5 \times 10^{-3} \mathrm{~m}$. The temperature range and ramp rate are representative for typical NanoTA measurements.

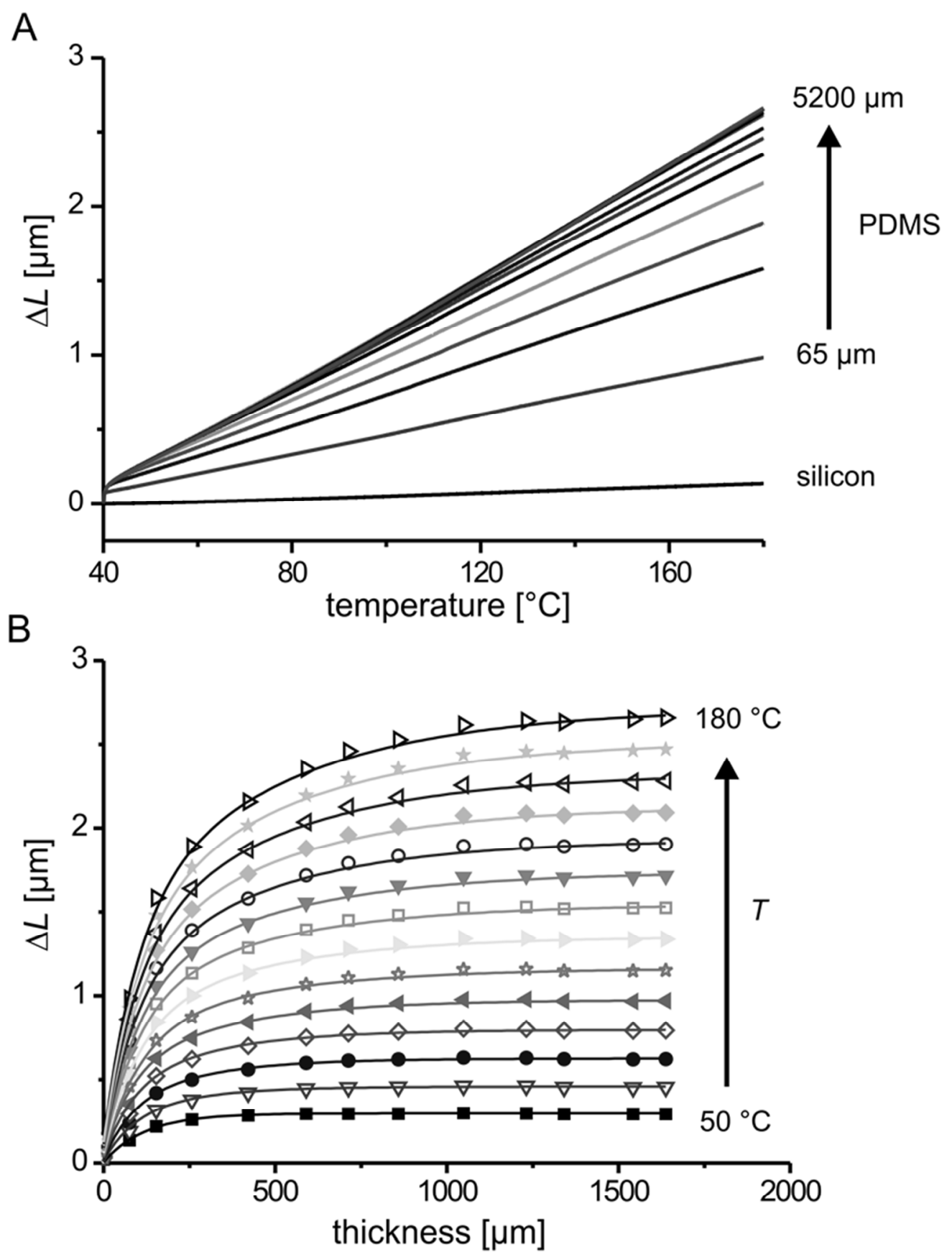

Figure 3.8: A) Thermal expansion vs. temperature plots obtained with heated AFM probe temperature scans from $40{ }^{\circ} \mathrm{C}$ up to $180^{\circ} \mathrm{C}$ with a temperature ramp of $10^{\circ} \mathrm{C} \mathrm{s}^{-1}$ on PDMS films with different thicknesses. B) Thermal expansion vs. PDMS film thickness for temperatures ranging from $50{ }^{\circ} \mathrm{C}$ up to $180{ }^{\circ} \mathrm{C}$ measured during the temperature ramps presented in $A$. The solid lines in $B$ are exponential fits to the data and serve as a guide for the eye. 
It can be seen in Figure 3.8A that the measured thermal expansion is very small for a bare silicon surface, whereas it increases significantly, when PDMS films are locally heated. Finally, for the thicker films the thermal expansion becomes independent of the film thickness. The PDMS film thickness at which this occurs was calculated from a plot of the observed thermal expansion as a function of the PDMS film thickness (Figure 3.8B). The thermal expansion data were plotted for temperature increments of $10{ }^{\circ} \mathrm{C}$. $L_{i s o}$ was determined for each corresponding temperature from the exponential fits of the experimental data. Figure 3.9 shows $L_{i s o}$ for the respective temperatures with an interval of $10{ }^{\circ} \mathrm{C}$. During the applied non steady state localized surface heating, the length scale of non steady state thermal conduction in the PDMS films directly below the heated cantilever ranges from $\sim 465 \mu \mathrm{m}$ up to $\sim 1350 \mu \mathrm{m}$. The fit to the data suggests that $L_{i s o}$ is proportional to $T^{0.33}$ within the temperature range investigated.

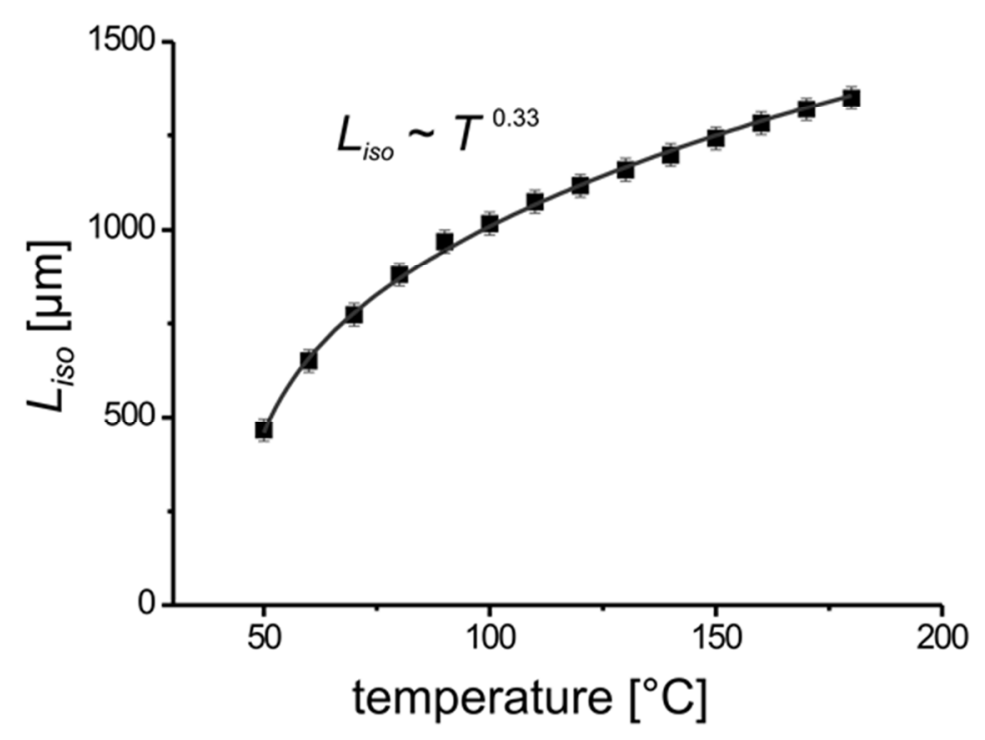

Figure 3.9: $L_{\text {iso }}$ as a function of temperature during NanoTA temperature ramps on PDMS films from $40^{\circ} \mathrm{C}$ to $180^{\circ} \mathrm{C}$ at a heating rate of $10^{\circ} \mathrm{C} \mathrm{s}^{-1}$.

The exact temperature profile at these length scales is unknown, however, a steep thermal gradient close to the probe-sample contact point can be expected. ${ }^{3,35}$ Despite the unknown exact thermal gradient, the averaged temperature increase is expected to be on the order of approximately $10{ }^{\circ} \mathrm{C}$, based on the measured thermal expansion of the thicker PDMS samples heated to $180{ }^{\circ} \mathrm{C} .{ }^{36}$ The length scale of heat transport was verified in measurements with $100 \mu \mathrm{m}$ diameter thermocouples embedded in PDMS samples at $0 \mu \mathrm{m}, 500 \mu \mathrm{m}$ and $1150 \mu \mathrm{m}$ depth, respectively. Upon increasing the probe temperature $\left(T_{i}\right)$ from $40{ }^{\circ} \mathrm{C}$ to $180{ }^{\circ} \mathrm{C}$ at a heating rate $10{ }^{\circ} \mathrm{C} \mathrm{s}^{-1}$ the embedded thermocouple detected a measureable increase in temperature (data not shown). 
Subsequent heating and cooling cycles did not show any hysteresis. Despite the semiquantitative nature of the data acquired the observed thermocouple response at depths over $1000 \mu \mathrm{m}$ confirms the observed length scale of heat transport for the measured $L_{i s o}$ calculated from the thermal expansion data on PDMS. The normalized temperature response for the depths investigated revealed a sharp drop in temperature close to the tip-sample contact, which is in agreement with our expectations. Interestingly, the thermocouples also showed an increase in temperature, when they were embedded at a depth of $500 \mu \mathrm{m}$, while the heated cantilever was positioned $100 \mu \mathrm{m}$ above the PDMS film (i.e. not in physical contact with the PDMS surface). No change in temperature was detected when the heated cantilever was positioned $1000 \mu \mathrm{m}$ above the PDMS surface. Furthermore, an increased temperature was observed, when the heated cantilever was in contact with the PDMS film and positioned $500 \mu \mathrm{m}$ away in the lateral $(x)$ direction from the initial contact point straight above the embedded thermocouple. These results once more confirm that for typical conditions used in NanoTA measurements heat transport in $z$-direction extends over distances $>1000 \mu \mathrm{m}$ for polymers.

\subsubsection{Simplified steady state 1D model}

The observation of the $133{ }^{\circ} \mathrm{C}$ isotherm at micrometer length scales from the tip-probe contact points for $T_{i}$ between $200{ }^{\circ} \mathrm{C}$ and $300{ }^{\circ} \mathrm{C}$ suggests that there is a very steep thermal gradient close to the tip, when it is in contact with the polymer film. This is confirmed by the calculated average temperature increase of $\sim 10{ }^{\circ} \mathrm{C}$ for the PDMS film, which extends over $1000 \mu \mathrm{m}$ in the $z$ direction for a probe temperature of $180{ }^{\circ} \mathrm{C}$. On the other hand, as discussed above, the PDMS as well as the PET experiments revealed that surface heating with the cantilever positioned slightly above the polymer films induces an increase in surface and sub-surface temperature. Therefore substantial surface heating at these distances must be taken into account. The existence of a plateau value in Figure 3.6 suggests that after 100 seconds a quasi steady state is reached for the pre-set probe temperatures used. These isothermal points at known distances from the tip-sample contact point were taken into account in a simplified 1D model for steady state spherical heat conduction (Figure 3.10). The model is based on the following assumptions:

- The system is in the steady state.

- The tip-PET interface is a hemisphere with pre-set temperature $\left(T_{i}\right)$.

- Phase changes can be neglected.

- Convective surface cooling can be neglected. 
A

AFM probe
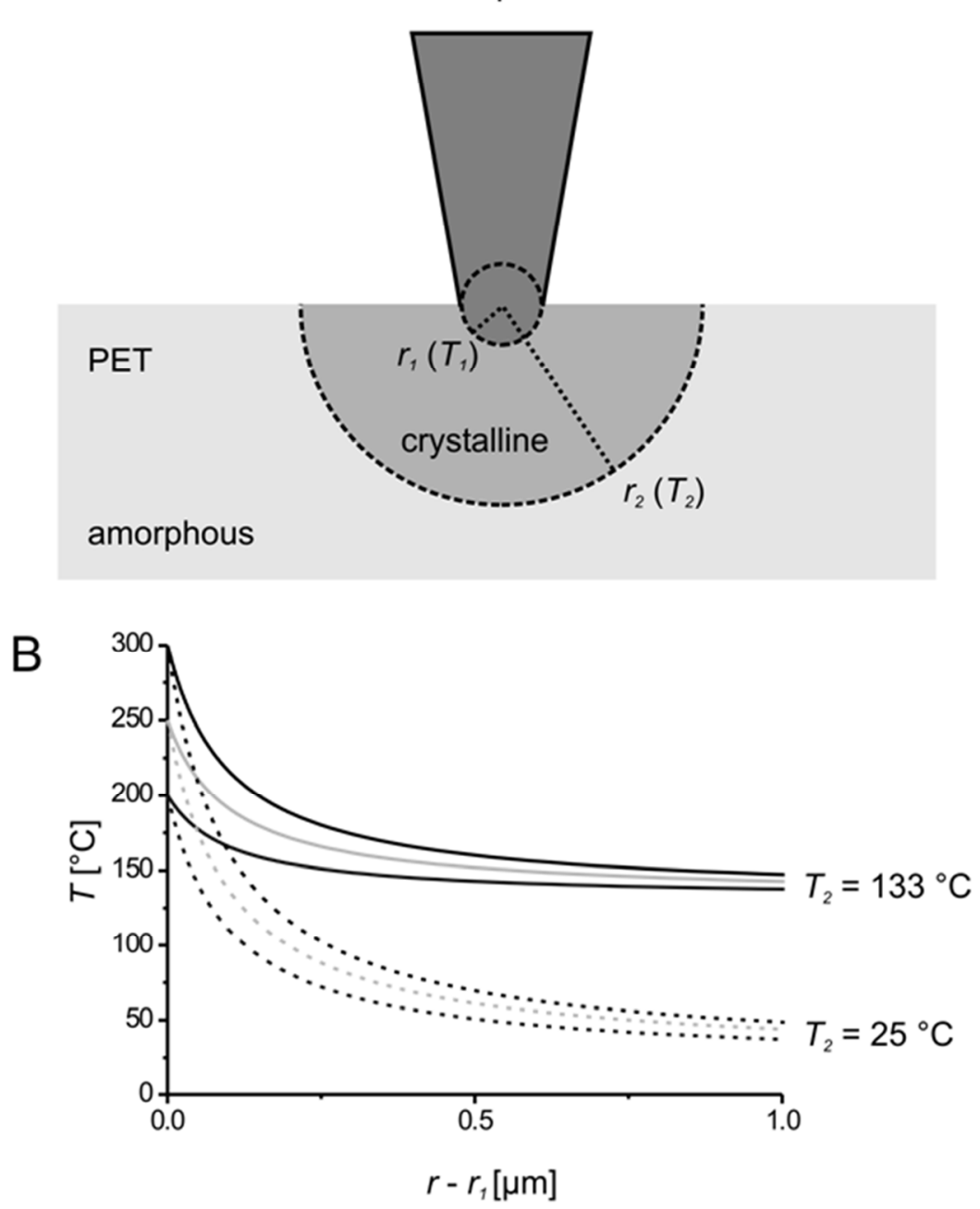

Figure 3.10: (A) Simplified schematic of a heated AFM probe in contact with an amorphous PET film (The tip size and the radii $r_{1}$ and $r_{2}$ are not drawn to scale). (B) Calculated steady state temperature profiles around a heated AFM tip with pre-set temperatures $\left(T_{1}=T_{i}\right)$ of $200{ }^{\circ} \mathrm{C}, 250{ }^{\circ} \mathrm{C}$ and $300{ }^{\circ} \mathrm{C}$. The radial distance away from the probe-polymer interface is represented by $r-r_{1}$. The solid lines represent the calculated temperature profiles, when surface heating $\left(T_{2}=133^{\circ} \mathrm{C}\right)$ in the vicinity of the tip is taken into account, whereas the dotted lines assume no significant surface heating in the proximity of the tip $\left(T_{2}=25^{\circ} \mathrm{C}\right)$.

The steady state heating rate $q$ conducted through the PET hemisphere can be described as follows: ${ }^{37}$

$q=\frac{2 \pi k r_{1} r_{2}}{r_{1}-r_{2}}\left(T_{1}-T_{2}\right)$

where $r_{1}$ and $r_{2}$ denote the radii at steady surface temperatures of $T_{1}$ and $T_{2}$, respectively with $T_{1}>T_{2}$ and $k$ is the thermal conductivity of PET $\left(0.24 \mathrm{~W} \mathrm{~m}^{-1} \mathrm{~K}^{-1}\right){ }^{38}$ 
The temperature gradient in the hemisphere can be calculated as:

$\frac{T-T_{2}}{T_{1}-T_{2}}=\frac{1-r_{2} / r}{1-r_{2} / r_{1}}$

where $r$ is the radius at temperature $T$ with $T_{1}>T>T_{2}$ and $r_{1}<r<r_{2}$.

In this model $r_{1}$ represents the tip-PET interface contact point assuming a spherical contact area. The contact radius $r_{1}$ was estimated from cross-sectional analysis of the probe polymer contact point observed in the AFM height images by fitting a spherical tip shape in the residual indent (see for example Figure 3.5F). AFM height images for heating times of 100 seconds and 120 seconds for all pre-set temperatures were taken into account. For $r_{l}$ a value of $220 \pm 40 \mathrm{~nm}$ was calculated. This value of $r_{l}$ is large compared to the typical tip radius stated by the manufacturer for these heatable AFM probes $(<30 \mathrm{~nm}$, see Chapter 2$)$. The discrepancy is explained by the fact that while growing the semi-crystalline domains the temperature is well above the $T_{g}$ of PET $\left(T_{g} \sim 73{ }^{\circ} \mathrm{C}\right)$. Hence the probe penetrates the polymer film substantially, resulting in an increased contact area. Analyzing AFM height images obtained by scanning an array of sharp tips ${ }^{39}$ with a new and an extensively used heatable AFM probe provided tip radii on the order of $30 \mathrm{~nm}$ and $100 \mathrm{~nm}$, respectively. Based on these numbers a value of $100 \mathrm{~nm}$ will be used for the following calculations. It is assumed that for most applications with these heatable AFM probes this number leads to an overestimate for the tip-sample contact area. The value of $r_{2}$ was calculated as $0.5 \times D_{\max }$. Figure $3.10 \mathrm{~B}$ shows the temperature distribution calculated from equation 2 for pre-set probe temperatures of $200{ }^{\circ} \mathrm{C}, 250{ }^{\circ} \mathrm{C}$ and $300{ }^{\circ} \mathrm{C}$ with and without taking substantial surface heating into account. The use of the isothermal boundary at $0.5 \times D_{\max }$ to calculate the thermal gradient from the tip-sample interface is justified based on the observed micrometer range crystallization of PET, when there was no contact between the heated cantilever and PET. Furthermore for an increased $r_{2}$ up to $1000 \mu \mathrm{m}$ no significant change in the thermal gradient close to the tip-PET contact point was observed, which once more justifies its use as a boundary condition in our calculations.

In Figure 3.10B a very sharp decrease in temperature is observed close to the tip-polymer contact point regardless whether substrate heating is taken into account or not. Approximately $90 \%$ of the temperature drop occurs within $1 \mu \mathrm{m}$ from the tip-polymer contact point. Less steep thermal gradients are observed when substantial surface heating is taken into account. This will adversely influence the resolution of 
thermal scanning probe based material characterization or manipulation. The heat flux from the heated probe through the tip-polymer interface $\left(q_{i}\right.$, Figure 3.1) was calculated to be $\sim 1.4 \%, \sim 1.1 \%$, and $\sim 0.8 \%$ of the power generated in the heater area in the cantilever end at a tip temperature of $300{ }^{\circ} \mathrm{C}, 250{ }^{\circ} \mathrm{C}$ and $200{ }^{\circ} \mathrm{C}$, respectively. These numbers are in reasonable agreement with calculations from King and coworkers in which $0.1 \%$ of the power generated was found to travel through the probe-material interface. ${ }^{1}$ Lowering of the large contact radius $\left(r_{l}\right)$ to e.g. $30 \mathrm{~nm}$ will substantially lower the calculated heat flux through the tip-polymer interface to approximately $0.3 \%$ of the total power generated at the cantilever heater area $\left(T_{i}=250{ }^{\circ} \mathrm{C}\right)$.

This simplified model does not allow one to model the thermal transport completely or to predict accurate thermal gradients. However the experimental results presented above revealed long range heat transport over more than $1000 \mu \mathrm{m}$ within polymeric materials under typical NanoTA conditions. Short contact times at relatively low temperatures already revealed thermal transport over several hundreds of micrometers and the existence of a very sharp temperature gradient close to the tip.

Several examples are known in literature on how to avoid substantial surface heating in different research areas, where heatable AFM cantilevers are used. First we would like to mention the somewhat trivial solution for thermal scanning probe lithography, which is fast scanning while reducing the contact area of the tip with the polymer material. ${ }^{40}$ Secondly in applications such as nanoDSC the application of an AC current instead of a DC current to the cantilever induces an oscillating temperature that significantly reduces the thermal transport in the surface below the heated cantilever. ${ }^{41}$

The experimental results presented here could be used in future research to further improve (existing) models for thermal transport from heated AFM cantilevers in contact with polymer surfaces, as well as in optimizing the experimental conditions for heated cantilever based AFM approaches. For instance, in Chapter 4 the effect on $T_{i}$ of good thermally conductive substrates in proximity of the probe tip-sample contact interface for thin polymer films will be described in detail.

\subsection{Conclusions}

The length scale of thermal transport in both lateral and vertical directions arising from heated AFM cantilevers in contact with polymer films was experimentally investigated. Heated probe induced crystallization of amorphous PET resulted in the formation of near circular semi-crystalline domains in the tip-near region. The periphery of the domain boundaries was considered as a surface isotherm at $133{ }^{\circ} \mathrm{C}$. The radii of the observed lateral surface isotherms ranged from $2.2 \pm 0.5 \mu \mathrm{m}$ up to 
$18.7 \pm 0.5 \mu \mathrm{m}$ as was revealed with AFM and polarized light microscopy for heated AFM probe tip temperatures between $200{ }^{\circ} \mathrm{C}$ and $300{ }^{\circ} \mathrm{C}$ and contact times varying from 20 seconds up to 120 seconds. The heat transport into polymer films was estimated from thermal expansion measurements of silicon supported PDMS films with variable thickness. Our data showed that heat transport in the $z$ direction occurred to depths over $1000 \mu \mathrm{m}$ using representative non steady state SThM conditions (i.e. heating from $40{ }^{\circ} \mathrm{C}$ to $180{ }^{\circ} \mathrm{C}$ at $10{ }^{\circ} \mathrm{C} \mathrm{s}^{-1}$ ). This was semiquantitatively confirmed by temperature measurements with thermocouples embedded in PDMS films at depths comparable to the observed length scale of thermal transport. A simplified model for 1D steady state spherical heat transport showed a steep temperature gradient close to the tip contact point. The temperature gradient was less steep, when surface heating was taken into account. The experimental evaluation of thermal transport from heated AFM cantilevers in contact with polymer films will be of use for the further development of heated cantilever based AFM approaches as well as for the validation of theoretical models.

\subsection{Experimental}

PDMS film preparation: The films were prepared from Sylgard-184 (Dow Corning, Midland, MI, USA). Typically 5:1 (w/w) elastomer to curing agent mixtures were degassed by applying a vacuum for 20 minutes in two subsequent pumping cycles before pouring them carefully over a silicon wafer $\left(10 \mathrm{~cm}\right.$ diameter, tilted at $\sim 12^{\circ}$, or on horizontally leveled $1 \mathrm{~cm} \times 1 \mathrm{~cm}$ silicon pieces). Following two more degassing cycles the PDMS was cured at $60^{\circ} \mathrm{C}$ for 12 hours. After cooling to room temperature the wafer was broken in the middle and marks were made with a scalpel at regular distances for positioning purposes. Copper-constantan thermocouples (5TC-TT-T-3672, Omega Engineering Inc. Stamford, CT, USA) were embedded in PDMS films at well defined positions by placing them in the prepolymer solution prior to curing to enhance the thermal contact. The exact depth of the thermocouple with respect to the PDMS surface was determined by cross-sectional light microscopy following the NanoTA measurements.

PET sample preparation: PET granulate (kind gift from Goodfellow, Huntingdon, UK), deposited between two pre-cleaned microscope glass slides, was molten at $290{ }^{\circ} \mathrm{C}$ in a temperature controlled hydraulic press (Specac, London, UK), while applying a load of $\sim 0.1$ ton. After cooling down the sample, the top microscopy slide was removed and the remaining PET film was heated again to $290{ }^{\circ} \mathrm{C}$ for 5 minutes to erase the thermal history, before it was quenched to room temperature by quickly removing the film from the hot press and placing it in MilliQ water (Millipore 
Synergy system, Billereca, MA, USA). The amorphous PET film was blown dry with a nitrogen stream. The thickness of the resulting PET film was over $1 \mathrm{~mm}$.

Atomic force microscopy: AFM measurements were done with a Dimension D3100 AFM operated with a Nanoscope IVa controller (Digital Instruments/Veeco, Santa Barbara, CA, USA) equipped with heatable silicon AFM probes (type AN-2, Anasys Instruments, Santa Barbara, CA, USA). The probe temperature was controlled with a NanoTA2 controlled (Anasys Instruments). Prior to the experiments the probe temperature was calibrated using polymer melting point standards ${ }^{42}$ poly( $\varepsilon$-caprolactone), poly(ethylene) and poly(ethylene terephthalate) with melting points of $55{ }^{\circ} \mathrm{C}, 116{ }^{\circ} \mathrm{C}$ and $235{ }^{\circ} \mathrm{C}$, respectively). Tip apexes were characterized using a silicon calibration grating with ultra sharp tips (nominal radius $10 \mathrm{~nm}$, TGT1, NT-MDT, Moscow, Russia). ${ }^{39}$

Thermal expansion measurements: The thermal expansion of the PDMS film was measured by recording the $\mathrm{Z}$-sensor position as a function of temperature while $T_{i}$ was ramped from $50{ }^{\circ} \mathrm{C}$ to $180{ }^{\circ} \mathrm{C}$ with a rate of $10{ }^{\circ} \mathrm{C} \mathrm{s}^{-1}$ at preselected positions of the sample. During the measurements a contact load of $\sim 10 \mathrm{nN}$ was applied.

Heat-induced crystallization: The local crystallization of PET films was carried out by positioning the heated AFM probe (with $T_{i}$ ranging from $200{ }^{\circ} \mathrm{C}$ to $300{ }^{\circ} \mathrm{C}$ ) in contact with the PET film with an initial contact force of $\sim 10 \mathrm{nN}$ for a specified holding time (ranging from 0 seconds up to 120 seconds). Afterwards the probe was withdrawn from the sample and subsequently cooled to room temperature. Contact mode AFM images of the formed semi-crystalline domains were captured with the same probe as used in the heating experiments. Tapping mode images of the semi-crystalline domains were recorded with PointProbe ${ }^{\circledR}$ Plus silicon probes (PPP$\mathrm{NCH}$, Nanosensors, Neuchatel, Switzerland).

Differential scanning calorimetry: DSC measurements on PET bulk samples were carried out with a Perkin-Elmer DSC-7 (Waltham, MA, USA). Heating and cooling traces, between $30{ }^{\circ} \mathrm{C}$ and $300{ }^{\circ} \mathrm{C}$, of amorphous PET samples were recorded at $10{ }^{\circ} \mathrm{C} \min ^{-1}$.

Polarizing optical microscopy: Images of the PDMS films (cross sectional) and PET semi-crystalline domains were recorded with an Olympus BX60 microscope. The PET semi-crystalline domains were examined between crossed polars with a lambda plate ( $\gamma 530 \mathrm{~nm}$, U-TP 530, Olympus, Zoeterwoude, The Netherlands) inserted between the specimen and analyzer. 


\subsection{References and notes}

1 King, W. P.; Saxena, S.; Nelson, B. A.; Weeks, B. L.; Pitchimani, R. Nano Lett. 2006, 6, 2145.

2 Nelson, B. A.; King, W. P. Rev. Sci. Instrum. 2007, 78, 023702.

3 Zhou, J.; Berry, B.; Douglas, J. F.; Karim, A.; Snyder, C. R.; Soles, C. Nanotechnology 2008, $19,495703$.

4 Gotzen, N. A.; Van Assche, G.; Ghanem, A.; Van Ingelgem, Y.; Hubin, A.; Van Mele, B. J. Therm. Anal. Calorim. 2009, 95, 207.

5 Mueller, T. Mater. Today 2009, 12, 40.

6 Basu, A. S.; McNamara, S.; Gianchandani, Y. B. J. Vac. Sci. Technol. B 2004, 22, 3217.

7 Hua, Y. M.; Saxena, S.; Clifford, H.; King, W. P. J. Micro-Nanolith. Mem. 2007, 6, 023012.

8 Hua, Y. M.; King, W. P.; Henderson, C. L. Microelectron. Eng. 2008, 85, 934.

9 Chui, B. W.; Stowe, T. D.; Ju, Y. S.; Goodson, K. E.; Kenny, T. W.; Mamin, H. J.; Terris, B. D.; Ried, R. P.; Rugar, D. J. Microelectromech. Syst. 1998, 7, 69.

10 King, W. P. J. Micromech. Microeng. 2005, 15, 2441.

11 Mamin, H. J. Appl. Phys. Lett. 1996, 69, 433.

12 Vettiger, P.; Cross, G.; Despont, M.; Drechsler, U.; Dürig, U.; Gotsmann, B.; Häberle, W.; Lantz, M. A.; Rothuizen, H. E.; Stutz, R.; Binnig, G. K. Nanotechnol T. IEEE. 2002, 1, 39.

Vettiger, P.; Despont, M.; Drechsler, U.; Dürig, U.; Häberle, W.; Lutwyche, M. I.; Rothuizen, H. E.; Stutz, R.; Widmer, R.; Binnig, G. K. IBM J. Res. Dev. 2000, 44, 323.

14 Gotsmann, B.; Knoll, A. W.; Pratt, R.; Frommer, J.; Hedrick, J. L.; Dürig, U. Adv. Funct. Mater. 2010, 20, 1276.

15 Pires, D.; Hedrick, J. L.; De Silva, A.; Frommer, J.; Gotsmann, B.; Wolf, H.; Despont, M.; Dürig, U.; Knoll, A. W. Science 2010, 328, 732.

16 Szoszkiewicz, R.; Okada, T.; Jones, S. C.; Li, T. D.; King, W. P.; Marder, S. R.; Riedo, E. Nano Lett. 2007, 7, 1064.

17 Wang, D. B.; Kodali, V. K.; Underwood, W. D.; Jarvholm, J. E.; Okada, T.; Jones, S. C.; Rumi, M.; Dai, Z. T.; King, W. P.; Marder, S. R.; Curtis, J. E.; Riedo, E. Adv. Funct. Mater. 2009, 19, 3696.

18 Lee, W. K.; Whitman, L. J.; Lee, J.; King, W. P.; Sheehan, P. E. Soft Matter 2008, 4, 1844.

19 Duvigneau, J.; Schönherr, H.; Vancso, G. J. Langmuir 2008, 24, 10825.

20 Vancso G. J.; Schön, P.; Duvigneau, J. Microscopy and Analysis 2009, 23, 5.

21 Nelson, B. A.; King, W. P. Nanosc. Microsc. Therm. 2008, 12, 98.

22 Masters, N. D.; Ye, W. J.; King, W. P. Phys. Fluids 2005, 17, 100615.

23 Lee, J.; Wright, T. L.; Abel, M. R.; Sunden, E. O.; Marchenkov, A.; Graham, S.; King, W. P. J. Appl. Phys. 2007, 101, 014906.

24 Kim, K. J.; Park, K.; Lee, J.; Zhang, Z. M.; King, W. P. Sens. Actuators A 2007, 136, 95.

25 Häberle, W.; Pantea, M.; Hoerber, J. K. H. Ultramicroscopy 2006, 106, 678.

26 Kim, K. J.; King, W. P. Appl. Therm. Eng. 2009, 29, 1631.

27 Park, K.; Cross, G. L. W.; Zhang, Z. M. M.; King, W. P. J. Heat. Trans. T. ASME 2008, 130, 102401.

28 Schroeder, M. J.; Roland, C. M. Macromolecules 2002, 35, 2676.

29 Kong, Y.; Hay, J. N. Polymer 2003, 44, 623.

30 Righetti, M. C.; Di Lorenzo, M. L.; Tombari, E.; Angiuli, M. J. Phys. Chem. B 2008, 112, 4233.

31 Turner, P. S. J. Res. Nat. Bur. Stand. 1946, 37, 239.

32 Holliday, L.; Robinson, J. J. Mater. Sci. 1973, 8, 301. 


\section{Chapter 3}

33 Feltham, S. J.; Yates, B.; Martin, R. J. J. Mater. Sci. 1982, 17, 2309.

34 Shevchenko, E. G.; Malyutina, T. I.; Borina, V. K.; Kafel, L. M. Meas. Tech. 1986, 29, 866.

35 Liu, X. J.; Yang, Y. W.; Yang, J. P. J. Appl. Phys. 2009, 105, 013508.

36 The averaged temperature increase $(\Delta T)$ within the PDMS film was calculated according to $\Delta L$ $=\alpha_{L} \Delta T L_{0}$. In which $\alpha_{L}$ is the linear thermal expansion coefficient $\left(2 \times 10^{-4}{ }^{\circ} \mathrm{C}^{-1}\right)$ and $L_{0} \sim 1$ $\mathrm{mm} . \Delta L$ is the typically observed absolute thermal expansion for the thicker PDMS films at $T_{i} \sim$ $180^{\circ} \mathrm{C}$.

37 Rohsenow, W. M.; Hartnett, J. P.; Ganić, E. N. Handbook of Heat Transfer Fundamentals, 2nd ed.: McGraw-Hill Book Company, New York, 1985.

38 Van der Vegt, A. K.; Goveart, L. E. Polymeren: van Keten tot Kunstof, 5th ed.: Delft: VSSD, Delft, 2005.

39 Bykov, V.; Gologanov, A.; Shevyakov, V. Appl. Phys. A 1998, 66, 499.

40 Hua, Y.; Saxena, S.; Lee, J. C.; King, W. P.; Henderson, C. L. Proc. of SPIE 2007, 6517, $65171 \mathrm{~L}-1$.

41 Kuo, C.; Chen, C. C.; Bannister, W. Thermochim. Acta 2003, 403, 115.

42 Nelson, B. A.; King, W. P. Sens. Actuators A 2007, 140, 51. 


\section{Chapter 4 \\ NanoTA of Thin Polymer Films: Tip-Sample Interface Temperature Effects on the Onset of Indentation}

In this chapter the polymer film thickness dependent deviation of the effective probe tip-sample interface temperature $\left(T_{i}\right)$ from the calibrated $T_{i}$ during nanoscale thermal analysis (NanoTA) of supported films is described. Polymer films with thicknesses $(d)$ between $20 \mathrm{~nm}$ and $3.5 \mu \mathrm{m}$ on silicon or glass substrates were prepared by spin coating. The measured surface softening temperature with NanoTA ( $T_{\text {s NanoTA }}$ ) increased with decreasing film thickness values (for $d<<1 \mu \mathrm{m}$ ). This effect is due to enhanced power dissipation related to the heated probe tip being in close proximity of a substrate with a higher thermal conductivity $(\lambda)$ compared to the polymer film. Hence, the effective $T_{i}$ is significantly lowered as compared to the set value based on a calibrated $T_{i}$. For comparable film thicknesses this effect was more pronounced for films on silicon $\left(\mathrm{SiO}_{2}\right)$ substrates compared to films on glass substrates, as was expected from $\lambda_{S i}$ being approximately 100 times higher compared to $\lambda_{\text {glass }}$. In order to estimate the deviation in $T_{i}$ from the calibrated temperature $\left(\Delta T_{i}\right)$ the NanoTA results were compared with isothermally conducted nanoscale thermal mechanical analysis (nTMA) measurements. The calculated differences in $T_{s}$ measured with NanoTA and nTMA provide an estimate of $\Delta T_{i}$ as a function of the film thickness. It was observed that $\Delta T_{i}$ is on the order of several tens of degrees Celsius for films thinner than $100 \mathrm{~nm}$. The film thickness at which the temperature starts to deviate significantly from the surface $T_{s}$ of thick films $(d \geq 3.5 \mu \mathrm{m})$ taking experimental differences into account $\left(d_{a p p}\right)$ was calculated to be on the order of several hundreds of nanometers for poly(styrene) (PS), poly(methyl methacrylate) (PMMA) and polycarbonate (PC) films on $\mathrm{SiO}_{2}$. These results are in good agreement with the observed length scale of heat transport described in Chapter 3. Overall the obtained results for $\Delta T_{i}$ for silicon supported polymer thin films are useful for a better understanding of NanoTA measurements of thin polymer films. In addition, it is expected to be of use for the development of other scanning thermal microscopy approaches such as scanning thermal lithography. 


\subsection{Introduction}

Polymer (thin) films are widely researched and utilized in numerous areas of application, e.g. (bio)sensors, ${ }^{1-3}$ coatings $^{4-6}$ and semiconductors. ${ }^{7-9}$ The proximity of surfaces and interfaces can significantly affect polymer chain dynamics within the polymer film. Hence thin film properties usually differ from the respective bulk material properties. ${ }^{10-13}$ The performance of thin films in devices as well as during processing is among others determined by their thermodynamic stability and their thermal mechanical properties. Knowledge of the thermal transition temperatures as a function of the film thickness is therefore explicitly required. Unlike their corresponding bulk samples, polymer thin films cannot be analyzed with conventional thermal analysis techniques, such as differential scanning calorimetry (DSC), thermomechanical analysis (TMA) and dynamic mechanical analysis (DMA). Keddie, Jones and Cory ${ }^{14}$ were the first that reported on a systematic investigation of the film thickness $(d)$ dependent glass transition temperature $\left(T_{g}\right)$ measurements with ellipsometry. The $T_{g}$ was reported to decrease for decreasing film thicknesses of PS films at hydrogen passivated silicon substrates. Our group introduced a single molecule fluorescence method that allowed us to study the segmental chain dynamics in polymers. The fluorescence life time fluctuations of a fluorescent dye embedded in a polymer film were correlated to the number of segments involved in a local segmental rearrangement volume around the fluorescent probe. It was observed that for decreasing PS film thickness the number of segments involved in the segmental relaxation around the probe decreased, indicating an increase in polymer chain dynamics (i.e. reducing the $T_{g}$ ) ${ }^{15,16}$ In addition, Torkelson and coworkers ${ }^{17}$ have used a fluorescence method, in which a fluorescent dye labeled PMMA layer was embedded within a PMMA film at precisely known depths. The high sensitivity of the dye to miniscule changes in local density allowed the investigation of the structural relaxations at specified film depths. Their results showed for a $1000 \mathrm{~nm}$ thick PMMA film with $25 \mathrm{~nm}$ thick dye layer at known positions a decreased $T_{g}\left(\sim 5{ }^{\circ} \mathrm{C}\right)$ for a $25 \mathrm{~nm}$ dye PMMA layer at the air exposed interface and an increase in $T_{g}\left(\sim 12^{\circ} \mathrm{C}\right)$ at the substrate polymer interface. Dye layers separated more than $100 \mathrm{~nm}$ from any interface were reported to have $T_{g}$ values identical (within the experimental error) to the bulk $T_{g}$ values.

Due to its wide diversity and ease of applicability, atomic force microscopy (AFM) has a prominent status as a nanotechnology tool to study the morphology and material properties of surfaces. ${ }^{18-25}$ For instance, Briggs and coworkers ${ }^{26}$ have studied the film thickness dependent $T_{g}$ with localized TMA of isothermally heated PS films on silicon. In addition de Pablo and coworkers ${ }^{27}$ have investigated the film thickness dependent $T_{g}$ of PS films on silicon with heated Wollaston wire probes (see 
Chapter 2). Both reported on a significant decrease in $T_{g}$ for decreasing film thicknesses $(d<100 \mathrm{~nm})$. Measuring the surface $T_{g}$ by nTMA is considered to be time consuming whereas the use of Wollaston wire probes is limited by a lateral resolution exceeding $1 \mu \mathrm{m} .{ }^{28}$ Nanoscale thermal analysis (NanoTA) ${ }^{29,30}$ is an emerging AFM based nanotechnology tool for the fast and accurate determination of surface transition temperatures with high spatial resolution (see Chapter 2). NanoTA relies on the use of batch fabricated silicon AFM probes with an integrated resistive Joule heater embedded at the cantilever end. ${ }^{31,32}$ The utilization of these cantilevers with probe tips that have typical tip radii of $30 \mathrm{~nm}$ or less promises to yield access to surface transition temperature characteristics of materials with a spatial resolution of $100 \mathrm{~nm}$ or better. This is especially beneficial for the characterization of heterogeneous surfaces. Originally the described heatable AFM probe design was introduced for the development of high data-density data storage devices based on thermomechanical material manipulation. ${ }^{33,34}$ More recently, Knoll and coworkers have reported on the $3 \mathrm{D}$ pattering in polymer resists layers using scanning thermal lithography (SThL), demonstrating the possible high throughput of this patterning technique with $\sim 40 \mathrm{~nm}$ resolution. ${ }^{35,36}$ In addition to thermomechanical material manipulation, SThL has proven its feasibility in spatially controlled thermochemical surface functionalization and subsequent derivatization for the development of e.g. (bio)sensors. ${ }^{37-39}$ In the previous mentioned SThL applications polymer thin films were used as the reactive platform.

As described in Chapter 3 the thermal transport originating from the heated cantilever exceeds distances over 1 millimeter for the non-steady state heating conditions typically applied during NanoTA. In addition, significant surface heating below the heated cantilever usually takes place. Furthermore, a sharp temperature gradient within the first radial micrometer of the probe tip sample contact interface was demonstrated. Taking the results described in Chapter 3 into account, it is expected that the presence of solid supports for thin polymer films with thicknesses $<<1 \mu \mathrm{m}$ cannot be ignored in terms of probe tip-sample interface temperature $\left(T_{i}\right)$ and corresponding heat dissipation into the environment.

A critical issue to address here is that results reported on temperature controlled AFM based transition temperature measurements are all based on the thermomechanical probing of polymer indentation as a function of temperature (i.e. the onset of indentation is correlated to material softening). In addition, we would like to add that the $T_{g}$ is known to have a positive pressure dependency $\left(d T_{g} d P^{-1}\right)$. Typical values for polymeric materials of $d T_{g} d P^{-1}$ are in the range of $0.2-0.4{ }^{\circ} \mathrm{C} \mathrm{MPa}^{-1} \cdot{ }^{40}$ A simple estimation for the increase of $T_{g}$ underneath an AFM probe tip in contact 
with a polymer film with respect to the typical applied loads used throughout our NanoTA experiments resulted in an estimated increase of $\sim 10$ to $15{ }^{\circ} \mathrm{C}$. ${ }^{41}$ Hence it is our believe that the measured transition temperatures should be referred to as a softening temperature $\left(T_{s}\right)$ instead of a $T_{g}$. From this point on forward we will refer to the measured transition temperatures as $T_{s}$ when measured with temperature controlled AFM approaches and as $T_{g}$ when we speak of the properly measured bulk glass transition temperature.

Nelson and $\mathrm{King}^{29}$ reported on surface $T_{S}$ values of a $170 \mathrm{~nm}$ thick PS film on $\mathrm{SiO}_{2}$ substrates measured with NanoTA to be $\sim 20^{\circ} \mathrm{C}$ higher compared to the bulk $T_{g}$ determined from ellipsometry measurements. The measured deviation in $T_{s}$ was ascribed to the contact resistance for the heated tip and polymer film. Since they used Raman thermometry ${ }^{42}$ to calibrate the tip temperature (see Chapter 2), the contact resistance was not taken into account for the determination of $T_{i}$. Soles and coworkers ${ }^{43}$ have reported on the film thickness dependent $T_{s}$ of PS films on various supports. They used polymer melting point standards for the tip temperature calibration, assuming that the procedure takes the contact resistance into account (see Chapter 2). For supports with higher $\lambda$ values compared to PS they observed an increase in measured $T_{s}$ when decreasing the film thickness to $300 \mathrm{~nm}$ or lower. For a substrate with lower thermal conductivity the opposite trend was observed. They emphasized that the estimated threshold thickness was above the thickness at which deviations in surface $T_{s}$ from the bulk $T_{g}$ were to be expected. ${ }^{16}$ Finite element model simulations for a heated probe (represented by a heated sphere) in contact with thin PS films on $\mathrm{SiO}_{2}$ showed that sharper temperature gradients exist in thinner films. In addition, the calculated lower heat loss through the probe tip sample interface for thin films supported by glass compared to silicon supported films $\left(\lambda_{\text {glass }}<<\lambda_{S i}\right)$ pointed towards a significant effect of the proximity of solid supports on $T_{i}$. The deviation of $T_{i}$ for a heated probe tip in contact with a thin polymer film on a good thermally conductive substrate from the calibrated heated probe $T_{i}$ (which is used as a set value during the experiments) is referred to as $\Delta T_{i}$. Despite the systematic investigation and thorough discussion by Soles and coworkers, ${ }^{43}$ no experimental investigation of $\Delta T_{i}$ was provided.

For comparison, Briggs and coworkers, ${ }^{26}$ as previously described, have reported on the surface $T_{S}$ of PS films on glass to decrease with decreasing film thickness using AFM based localized TMA. They measured the hysteresis between the loading and unloading traces (i.e. a measure for indentation) of AFM force distance measurements as a function of temperature for isothermally heated samples. This approach will be referred to as nanoscale thermal mechanical analysis (nTMA). The temperature at which the depth of indentation starts to increase with increasing temperature is 
referred to as $T_{S} n T M A$. Since the samples were isothermally heated, $T_{i}$ was regarded as the set temperature. However unclear in their experimental design is whether the probe tip was also heated. No additional cantilever heating results in significant lowering of $T_{i}$. In this case the reported surface $T_{s}$ values by Briggs and coworkers are overestimated by a few degrees. The effect of "cold" tip induced surface cooling was demonstrated by Frank and coworkers. ${ }^{44}$ The estimated temperature depression under the tip in contact with a $100 \mathrm{~nm}$ thick PEO film maintained at $57^{\circ} \mathrm{C}$ was $\sim 15^{\circ} \mathrm{C}$.

Our experimental setup has a cantilever heater embedded in the tip holder in order to minimize the heat flux between the heated probe tip and the isothermally heated surface. In other words $T_{i}$ can be regarded as the set temperature. Hence, comparison of NanoTA and nTMA data obtained for the same polymer films provides insight in $\Delta T_{i}$ (see Section 4.2.1).

This chapter describes the systematic experimental determination of $\Delta T_{i}$ for NanoTA measurements of (thin) polymer films on $\mathrm{SiO}_{2}$ supports. Detailed insight in $\Delta T_{i}$ is of great importance for the accurate and precise thermal characterization and manipulation of materials with nanoscale thicknesses. Combined with Chapter 3 , in which the length scale of thermal transport was described, the work described in this chapter is implemented in the development of scanning thermal lithography (SThL) described in Chapter 7 and Chapter 8.

\subsection{Results and discussion}

Polymer thin films of PS, PMMA, PC and poly(vinyl chloride) (PVC) were prepared by spin casting from solutions on $15 \times 15 \mathrm{~mm}^{2}$ glass and silicon substrates. By controlling the spin speed (between $1000 \mathrm{rpm}$ to $2000 \mathrm{rpm}$ ) and the polymer concentration in the used solutions $(\sim 1 \mathrm{wt} \%-15 \mathrm{wt} \%)$ the film thickness was controlled between $20 \mathrm{~nm}$ and $3.5 \mu \mathrm{m}$. Film thicknesses were determined by tapping mode AFM step height measurements, as well as using null ellipsometry. The thickness values obtained with the two methods were in good agreement. Hence, for the following calculations and data processing the film thickness values calculated from the AFM step height measurements were used.

\subsubsection{NanoTA: substrate thermal conductivity effect}

NanoTA measurements of films with varying thickness on glass and silicon substrates were performed to determine $T_{S \text { NanoTA }}$ as a function of the film thickness. The $T_{S \text { NanoTA }}$ values correspond to the calibrated $T_{i}$ values. Figure 4.1 shows NanoTA graphs for PS films on silicon with thickness values between $100 \mathrm{~nm}$ and $2 \mu \mathrm{m}$. The corresponding $T_{S \text { NanoTA }}$ values were determined from the relative deflection versus 
temperature graphs as the temperature for which the slope equals zero (indicated by 0 in Figure 4.1). Obviously, for decreasing thickness values the measured $T_{S}$ NanoTA increases.

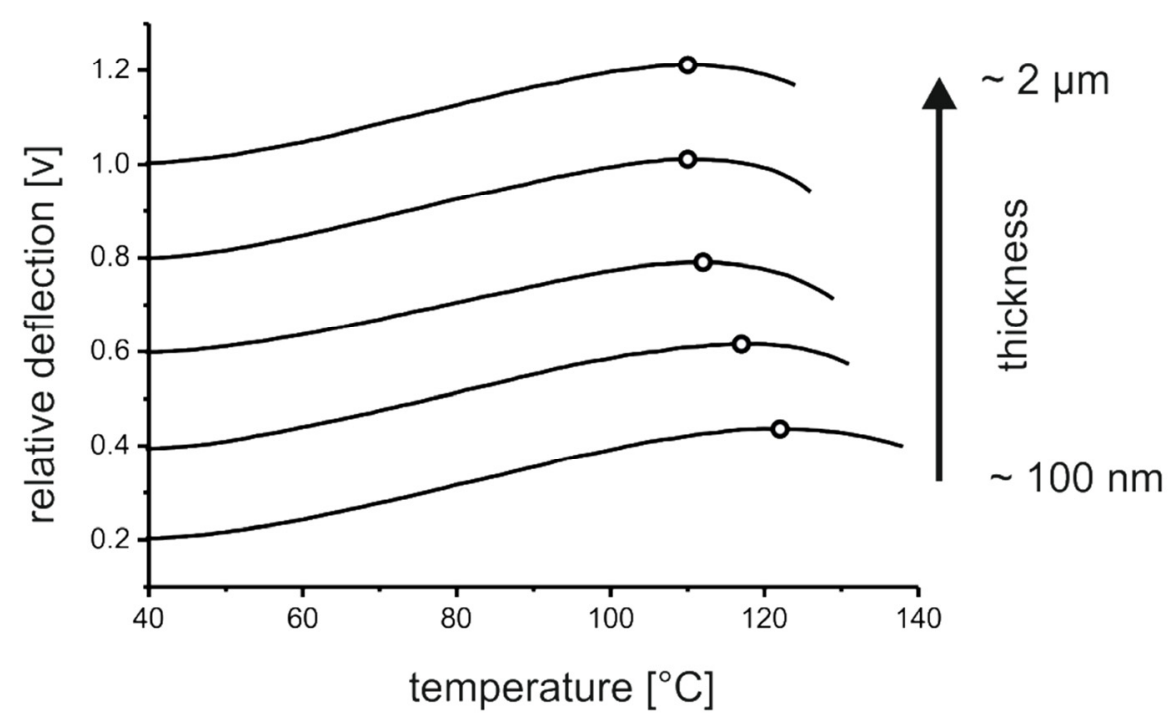

Figure 4.1: Relative deflection versus $T_{i}$ graphs measured by NanoTA for PS films with varying thicknesses on silicon. The maximum of the relative deflection versus temperature graphs indicated by 0 was identified as $T_{\text {s NanoTA. }}$

We note that with NanoTA measurements, softening of polymeric materials underneath the heated probe tip is measured. However the onset of the actual $T_{s}$ is expected at lower effective temperatures compared to $T_{S \text { NanoTA }}$ as determined from plots like Figure 4.1. Nelson and King ${ }^{29}$ nicely addressed this issue. They reported on NanoTA measurements on a PS film in which the temperature was increased stepwise with $3{ }^{\circ} \mathrm{C}$ per step, having an average temperature ramp rate of $30{ }^{\circ} \mathrm{C} \mathrm{min}{ }^{-1}$, while recording the probe resistance and the cantilever deflection. A decrease in resistance was observed at a temperature approximately $10{ }^{\circ} \mathrm{C}$ below the estimated transition temperature based on the deflection signal (i.e. $T_{S \text { NanoTA }}$ ). This indicated that the probe tip started to penetrate the polymer at a lower temperature compared to $T_{S \text { NanoTA. }}$.

Figure 4.2 shows the measured $T_{S \text { NanoTA }}$ as a function of the film thickness for PS, PMMA, PC and PVC films on both silicon (open squares) as well as on glass (open circles) substrates. The measured bulk $T_{g}$ with DSC is represented by the horizontal dashed line for each polymer. The general trend observed is that for decreasing film thickness the measured $T_{S}$ NanoTA increases. In addition, the measured increase was higher for silicon supported films compared to glass supported films. The measured $T_{s}$ NanoTA was higher compared to the bulk $T_{g}$ measured with DSC for all films. 
These observations are in agreement with the previously described results reported by Soles and coworkers. ${ }^{43}$ The measured increase in $T_{s}$ NanoTA for thinner films is ascribed to the presence of substrates with a higher $\lambda$ compared to the polymers used, in proximity of the probe tip-sample contact interface. In other words, more heat is transported away from the tip contact point, effectively decreasing $T_{i}$. Hence, for thinner films the measured $T_{S \text { NanoTA }}$ (i.e. the corresponding calibrated $T_{i}$ ) is higher than the effective $T_{i}$. Polymers ${ }^{45}$ typically have $\lambda$ values on the order of $0.1-0.2 \mathrm{~W} \mathrm{~m}^{-1} \mathrm{~K}^{-1}$ whereas glass ${ }^{46}$ and silicon ${ }^{47}$ have a $\lambda$ value of approximately $0.7-2.2 \mathrm{~W} \mathrm{~m}^{-1} \mathrm{~K}^{-1}$ and $156 \mathrm{~W} \mathrm{~m}^{-1} \mathrm{~K}^{-1}$, respectively. The roughly 100 times higher $\lambda$ for silicon compared to glass results in higher $T_{S \text { NanoTA for silicon supported films }}$ compared to films on glass substrates with similar film thicknesses.

The solid lines in Figure 4.2 are fits to the data according to the following empirical equation:

$$
T_{s}(d)=T_{s}^{\infty}\left[1 \pm\left(\frac{A}{d}\right)^{B}\right]
$$

In which $T_{s}(d)$ is the film thickness $(d)$ dependent $T_{s}, T_{s}{ }^{\infty}$ is the $T_{s}$ for infinite thick films and $\mathrm{A}$ and $\mathrm{B}$ are fitting parameters. This equation was used to describe the

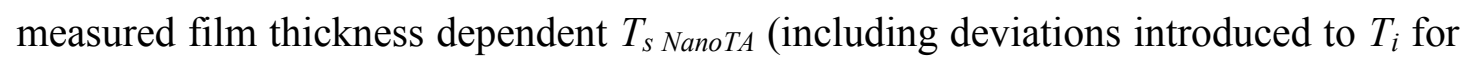
thinner film thickness values) and the obtained fits to the data were used in subsequent calculations for $\Delta T_{i}$ as a function of the film thickness. We note that this formula was used to fit the thickness dependent real $T_{g}$ depression by Jones and coworkers. ${ }^{14}$ In the context of our work the fit parameters have no direct physical meaning. 

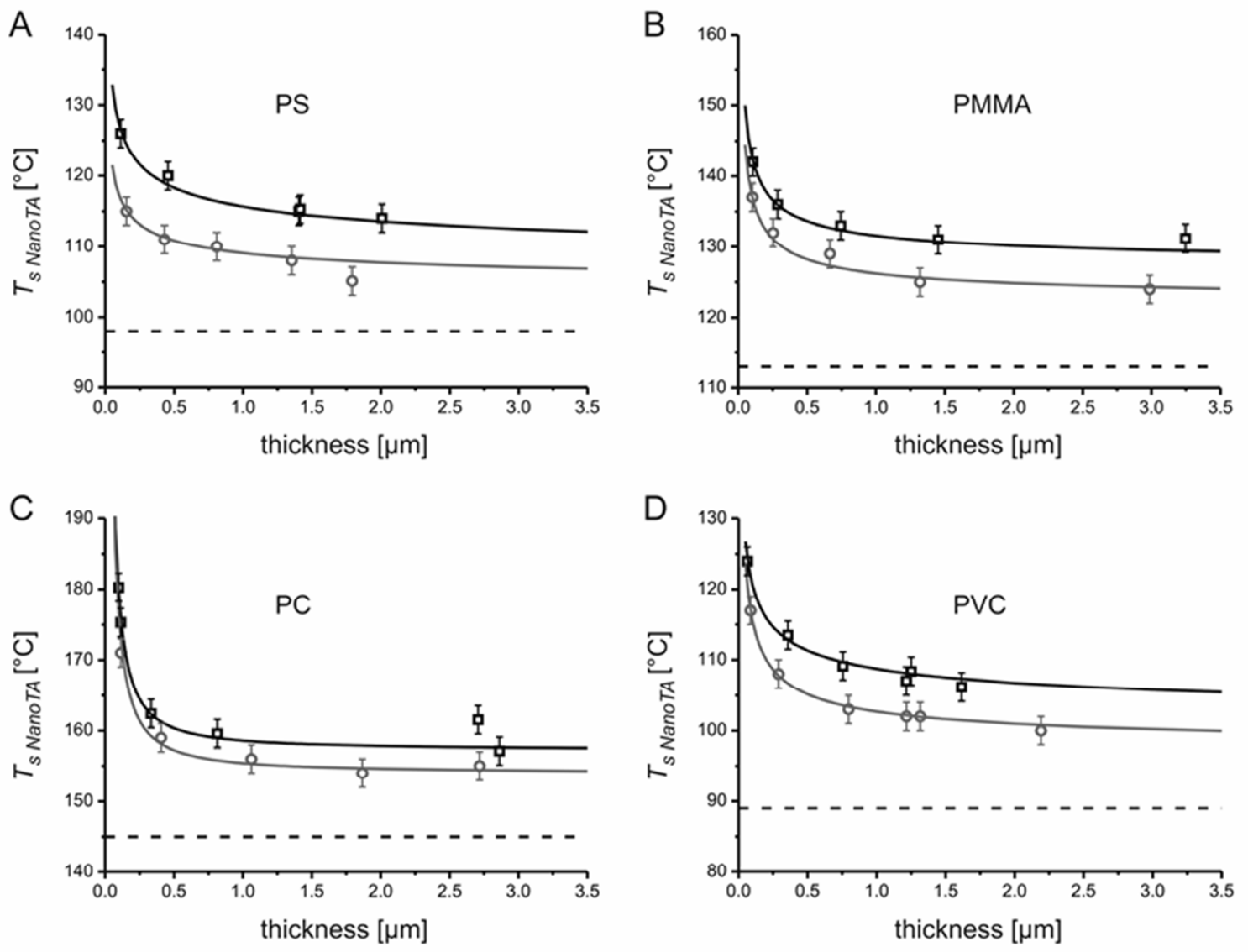

Figure 4.2: Measured $T_{s}$ NanoTA versus film thickness for PS (A), PMMA (B), PC (C) and PVC (D) films on silicon (open squares) and glass (open circles) substrates. The horizontal dashed line represents the bulk $T_{g}$ as measured with DSC. The solid lines are fits to the data according to equation 1.

Figure 4.3 shows schematically the measured surface $T_{s}$ of an amorphous polymer with NanoTA and nTMA having opposite trends for decreasing film thicknesses. ${ }^{48}$ The differences in measured $T_{s}$ are a measure of $\Delta T_{i}$ according to:

$\Delta T_{i}(d)=\left(T_{\text {S NanoTA }(d)}-T_{\text {SnTMA }(d)}\right)-\left(T_{\text {SNanoTA }(d=3.5 \mu \mathrm{m})}-T_{\text {SnTMA }(d=3.5 \mu \mathrm{m})}\right)+x$

The first term in equation 2 corresponds to the difference between the surface $T_{s}$ for a specific thickness measured with NanoTA and nTMA. The second term is a correction for experimental differences between both techniques based on the assumption that there is no difference in surface $T_{s}$ for $3.5 \mu \mathrm{m}$ thick films regardless of the used experimental technique to measure $T_{s}$. Finally, $x$ is an estimated correction term for differences in measured $T_{s}$ for films on $\mathrm{SiO}_{2}$ and glass substrates due to thermal transport over $3.5 \mu \mathrm{m}$ thick films decreasing $T_{i}$. The value of $x$ will be estimated further on. 


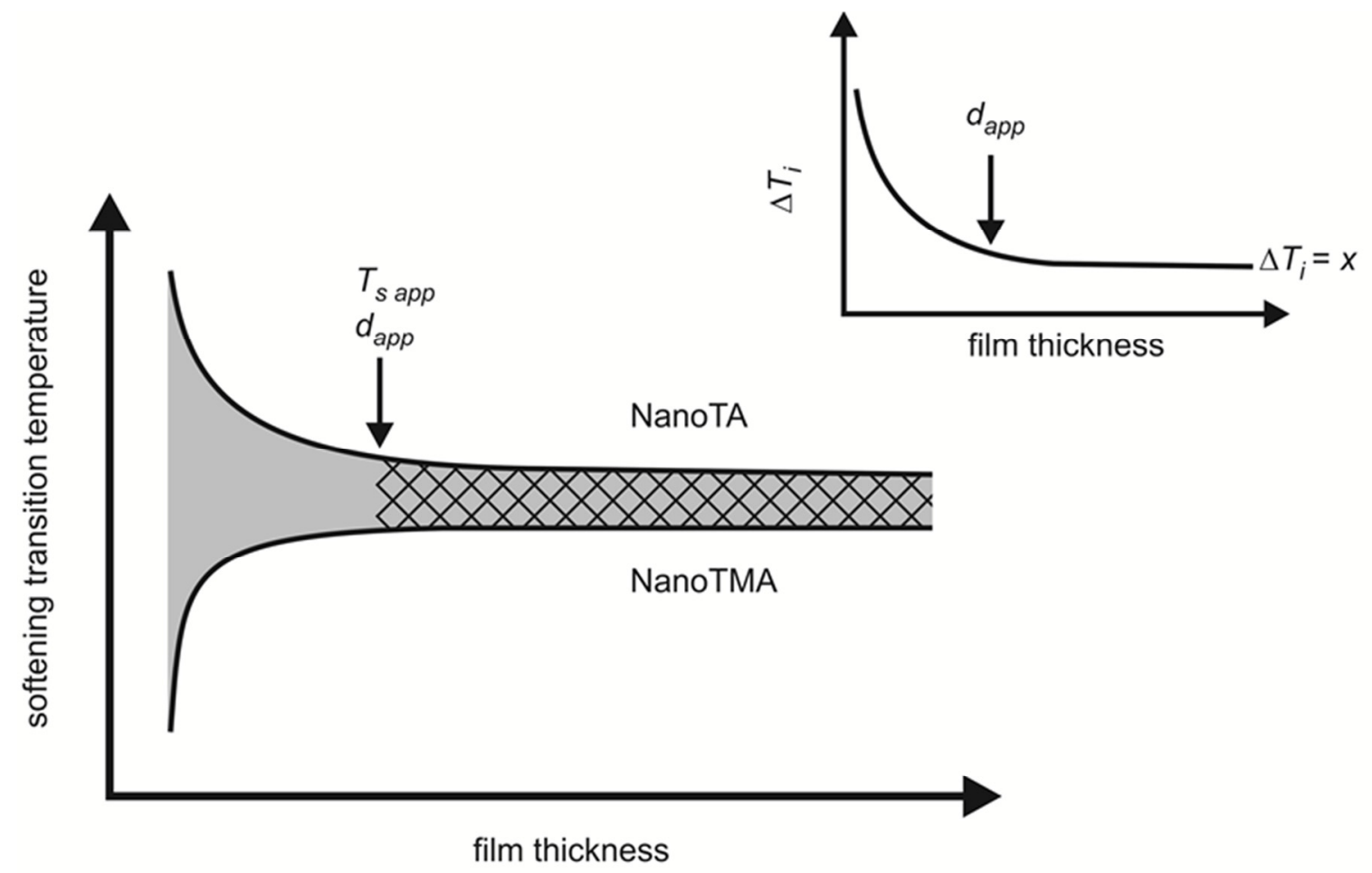

Figure 4.3: Schematic of the surface $T_{s}$ measured with NanoTA and nTMA as a function of the film thickness. The black arrow indicates the position of $d_{a p p}$ and $T_{s}$ app, respectively. The inset shows $\Delta T_{i}$ as a function of the film thickness. The differences observed for film thicknesses above $d_{\text {app }}$ (patterned filled area) are ascribed to experimental differences other than changes in calibrated $T_{i}$ (NanoTA).

The film thickness at which $\Delta T_{i}=x+5{ }^{\circ} \mathrm{C}$ is referred to as the apparent film thickness $\left(d_{\text {app }}\right)$. The additional $5{ }^{\circ} \mathrm{C}$ is based on the performed calculations including error analysis. NanoTA measurements on films with thicknesses $\leq d_{\text {app }}$ result in the measurement of an apparent surface $T_{s}\left(T_{s \text { app }}\right)$. We note that via this approach $d_{a p p}$ is estimated and depending on the choice for $x, d_{a p p}$ will vary significantly. However, it is our intention to provide as accurate as possible an estimate of $d_{\text {app }}$ based on validated assumptions.

The observed differences in measured $T_{s}$ NanoTA for thicker films (Figure 4.2, $d>1 \mu \mathrm{m}$ ) can be explained by $i$ ) thermal transport over these distances decreasing $T_{i}$ and $i$ ) the thermal mechanical mechanism of probing thermal transitions with NanoTA (i.e. probe tip penetration upon material softening). Thermal transport over these distances is expected when taking the results described in Chapter 3 into account. Comparing thick films $(d \sim 3.5 \mu \mathrm{m})$ on silicon and glass for the polymers used here showed that the films on silicon have an approximately $5{ }^{\circ} \mathrm{C}$ higher $T_{S \text { NanoTA }}$ compared to those on glass. This $5{ }^{\circ} \mathrm{C}$ difference is solely ascribed to $\lambda_{S i}$ being approximately 100 times higher compared to $\lambda_{\text {glass }}$. The observed differences compared to the bulk $T_{g}$ values were on the order of $15{ }^{\circ} \mathrm{C}$ and $10{ }^{\circ} \mathrm{C}$ for silicon and 
glass supported films, respectively. Since $\lambda_{\text {glass }}$ and $\lambda_{\text {polymers }}$ are of comparable order of magnitude, thermal conductivity effects for thicker films on glass are expected to be irrelevant. Therefore for the films on silicon we assume $x$ in equation 2 to be $5{ }^{\circ} \mathrm{C}$. This assumption appears to be valid since for PC and PS pellets (very thick samples) the measured $T_{S}$ NanoTA was approximately $5{ }^{\circ} \mathrm{C}$ lower compared to the $T_{S}$ NanoTA calculated for $\sim 3.5 \mu \mathrm{m}$ thick films. The remaining $10{ }^{\circ} \mathrm{C}$ difference between $T_{S}$ NanoTA for films on both glass and silicon substrates compared to the measured $T_{g}$ (bulk DSC) is ascribed to the experimental procedure to derive $T_{s}$ by NanoTA as discussed earlier. Hence the preferred terminology to use $T_{s}$ instead of $T_{g}$ is confirmed.

\subsection{2 nTMA of PS, PMMA and PC on silicon substrates}

The higher deviation in $T_{i}$ is expected at higher temperatures (due to increased heat transport to the surrounding). Therefore nTMA was used to determine the corresponding $T_{S} n T M A$ for PS, PMMA and PC (i.e. the polymers with the highest $T_{s}$ ). Force displacement curves were collected using isothermally heated samples placed within an environmental cell, mounted in the AFM, equipped with a sample and cantilever heater. Heating both the cantilever as well as the sample to the same temperature, the heat flow through the tip is minimized. Hence $T_{i}$ is regarded as the prescribed sample temperature.
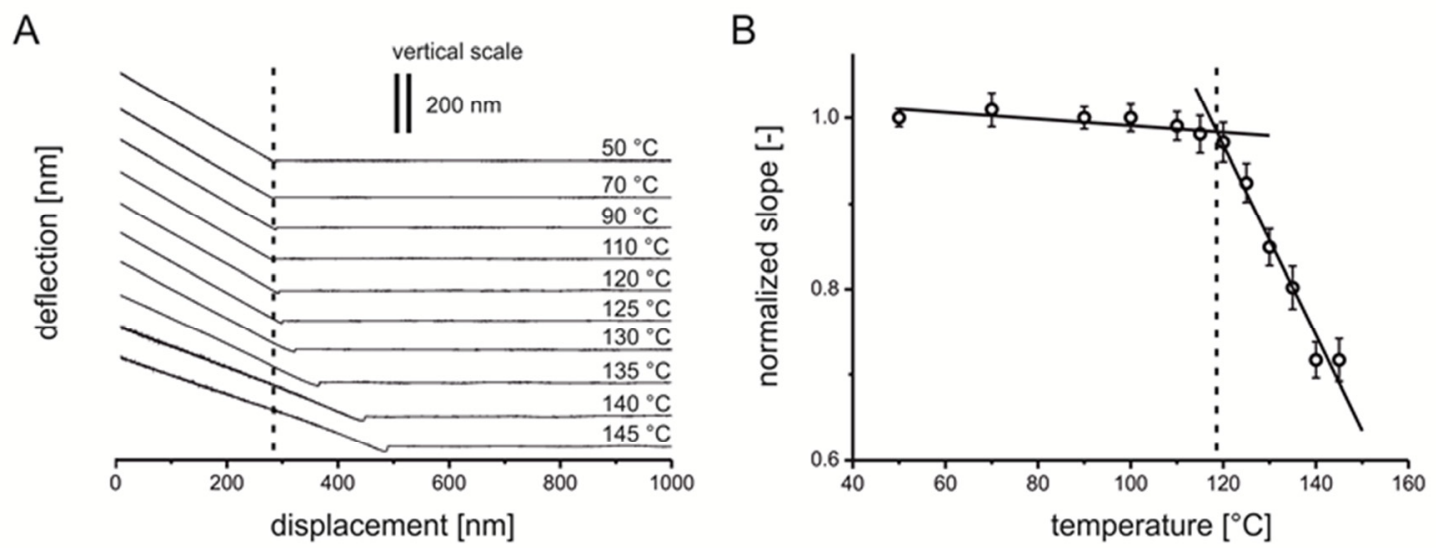

Figure 4.4: Approach part of force displacement curves recorded on a $1450 \mathrm{~nm}$ thick PMMA film on silicon at various temperatures (A). The averaged normalized slopes of the approach curves for the first $50 \mathrm{~nm}(n=25)$ in contact with the polymer films as a function of the temperature are shown in (B). The vertical dashed lines indicate the point of $T_{s}$ (i.e. $T_{\text {S ITMA }} \sim$ $\left.119^{\circ} \mathrm{C}\right)$.

Figure 4.4A shows the approach part of force displacement curves captured on a $1450 \mathrm{~nm}$ thick PMMA film on silicon at temperatures between $50^{\circ} \mathrm{C}$ and $145{ }^{\circ} \mathrm{C}$. When the cantilever is positioned free in air above the sample surface and is approaching the sample (lowering the displacement) no change in deflection is 
observed (horizontal part). Once the tip is in contact with the surface, the cantilever will bend upwards which is shown as an increase in the deflection signal. The slope of this part of the approach curve is for properly chosen cantilever spring constants $(k)$ a measure of the film's modulus for samples where the mechanical stress field is zero at the polymer-substrate interface. ${ }^{26,49}$

For temperatures below $T_{s}$ the modulus of the film is expected to be essentially constant, i.e. no significant changes in the measured slopes are expected. Upon increasing the temperature to above $T_{s}$, the modulus drops dramatically (typically around 3 orders of magnitude $)^{40}$ which is observed as a decrease in the measured slopes. This is demonstrated in Figure 4.4B, in which the slope normalized to the slope at $50{ }^{\circ} \mathrm{C}$ for the first $50 \mathrm{~nm}$ of the approach curve is plotted against temperature. The solid lines represent linear least square fits to the data below and above $T_{s}$. The calculated intersect of the two linear fits to the data at $\sim 119 \pm 3{ }^{\circ} \mathrm{C}$, is referred to as the $T_{S \text { nTMA. The calculated } T_{S} n T M A}$ is higher compared to the measured $T_{g}$ (bulk) value of $\sim 113{ }^{\circ} \mathrm{C}$ for PMMA. This can be ascribed to the increased pressure directly below the probe tip-sample contact interface.

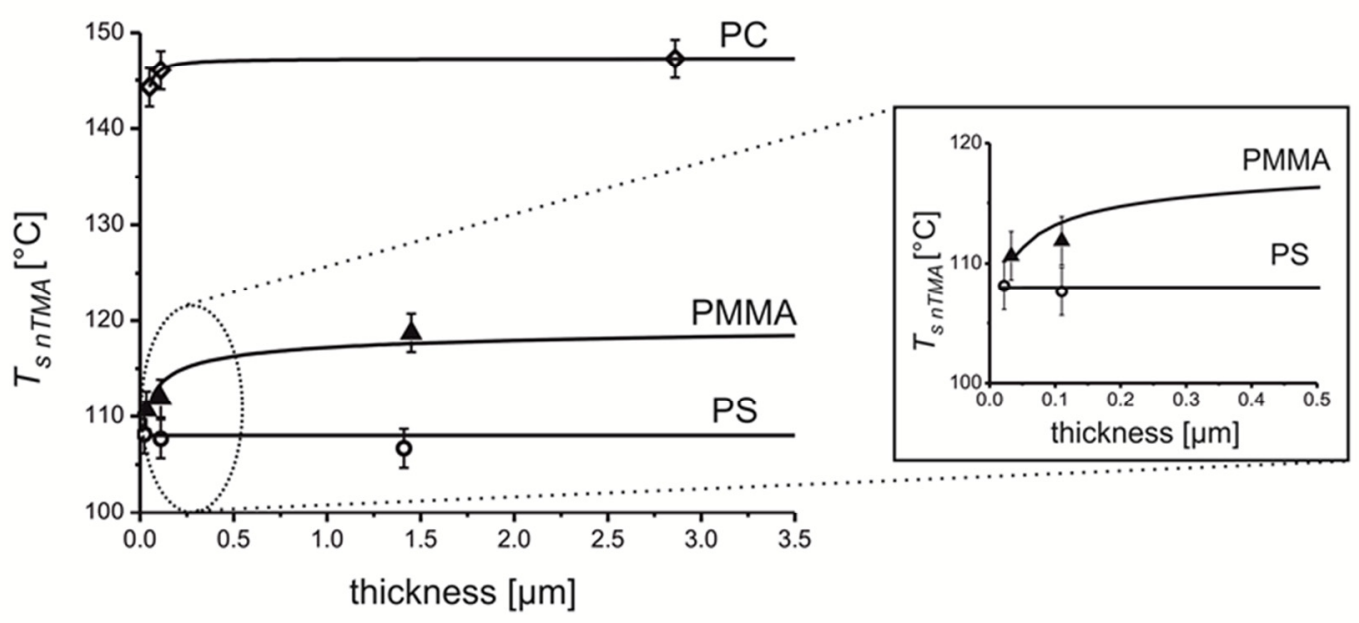

Figure 4.5: Measured $T_{\text {s nTMA }}$ versus film thickness for PS, PMMA and PC. The solid lines are fits to the data according to equation 1 and are a guide for the eye.

Figure 4.5 shows $T_{\text {s }}$ nTMA for PS, PMMA and PC for varying film thicknesses on silicon. For PC and PMMA a decrease in $T_{s} n T M A$ is observed for decreasing film thicknesses. For PS no change was observed. Comparison with the literature discussed previously ${ }^{26}$ showed that for PS also a decrease in measured $T_{S} n T M A$ was expected. Comparison of our data with data reported on in the literature is not trivial, since typically for studying the film thickness dependent $T_{s}$ of polymers well defined 
polymers were used (i.e. controlled molar mass with a monodisperse distribution). The molar masses of the polymers used in this chapter were measured with gel permeation chromatography (GPC, relative to PS calibration samples) to be $340 \mathrm{~kg}$ $\mathrm{mol}^{-1}, 35 \mathrm{~kg} \mathrm{~mol}^{-1}$ and $11 \mathrm{~kg} \mathrm{~mol}^{-1}$ with a polydispersity index (PDI) of 2.1, 1.8 and 3.4 for PS, PMMA and PC, respectively. The PDI values are much higher compared to typically reported values of $<1.10$ for the well-defined polymer systems. Therefore, in Section 4.2.4 the measured $T_{S \text { NanoTA }}$ and $T_{S n T M A}$ for two monodisperse (PDI 1.03) PS samples with a molar mass of $20 \mathrm{~kg} \mathrm{~mol}^{-1}$ and $650 \mathrm{~kg} \mathrm{~mol}^{-1}$, respectively, are shown.

\subsubsection{Estimation of $\Delta T_{i}$ for PS, PMMA and PC on silicon substrates}

Figure 4.6 shows the calculated $\Delta T_{i}$ as a function of the film thickness. The fitted functions through the data in Figures 4.2 and 4.5 were used to calculate $\Delta T_{i}$ according to equation 2 and $x=5{ }^{\circ} \mathrm{C}$. From Figure 4.6 the $d_{a p p}\left(\Delta T_{i}=10{ }^{\circ} \mathrm{C}\right)$ for PS, PMMA and PC were determined to be $670 \pm 87 \mathrm{~nm}, 680 \pm 88 \mathrm{~nm}$ and $340 \pm 44 \mathrm{~nm}$, respectively. For these film thicknesses the calculated errors were below $4{ }^{\circ} \mathrm{C}$. Hence the calculation of $d_{\text {app }}$ according to equation 2 with $x$ equals $5{ }^{\circ} \mathrm{C}$ and an additional $5{ }^{\circ} \mathrm{C}$ of overestimation for these numbers to be significantly meaningful, is justified. For $50 \mathrm{~nm}$ thick PS, PMMA and PC films $\Delta T_{i}$ was calculated to be $26 \pm 4{ }^{\circ} \mathrm{C}, 33 \pm 5{ }^{\circ} \mathrm{C}$ and $59 \pm 11^{\circ} \mathrm{C}$, respectively. The higher $T_{s}$ of the sample the larger the calculated $\Delta T_{i}$. This is attributed to more heat being transported away from the probe tip-sample contact interface at higher $T_{i}$ for heated probes in contact with polymer films with comparable film thicknesses.

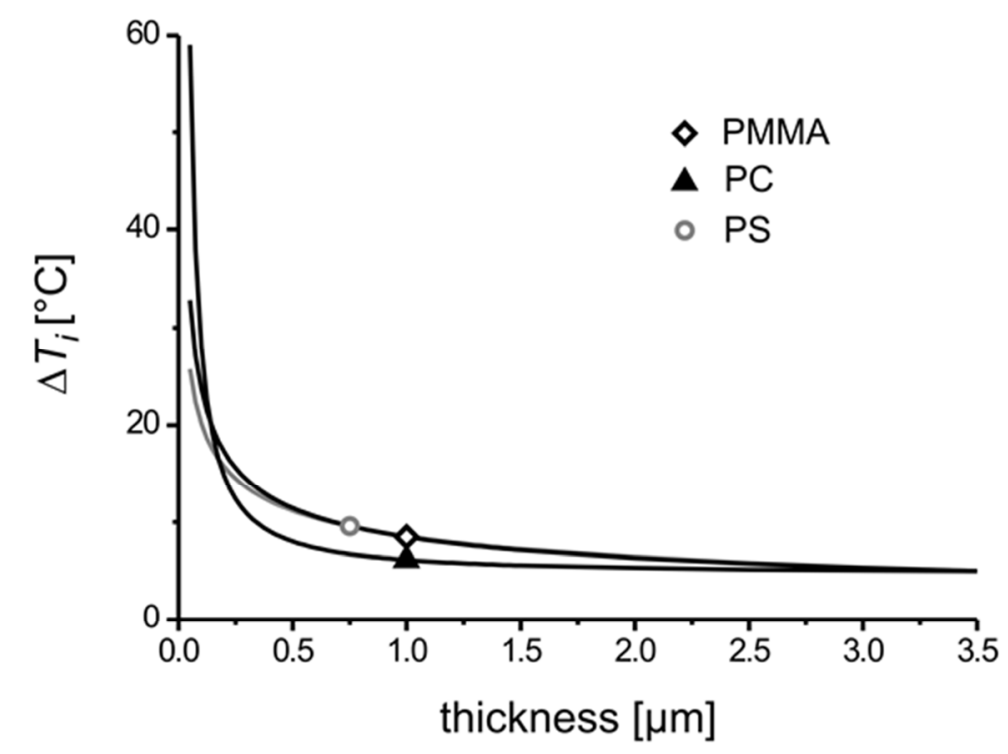

Figure 4.6: The calculated deviation in interface temperature as a function of the film thickness on silicon for PS, PMMA and PC. For $x$ in equation 2 a value of $5^{\circ} \mathrm{C}$ was used. 
PC has a significantly lower $d_{a p p}$ compared to those for PS and PMMA. This is attributed to the higher specific heat capacity for PC $\left(C_{p(300 \mathrm{~K})} \sim 306.8 \mathrm{~J} \mathrm{~mol}^{-1} \mathrm{~K}^{-1}\right)$ compared to PS and PMMA $\left(C_{p(300 \mathrm{~K})} \sim 127.4 \mathrm{~J} \mathrm{~mol}^{-1} \mathrm{~K}^{-1}\right.$ and $\sim 137.7 \mathrm{~J} \mathrm{~mol}^{-1} \mathrm{~K}^{-1}$, respectively). ${ }^{45}$ Polymer films with higher specific heat capacity values consume more cantilever heating power to increase the film temperature in proximity of the heated tip. Hence less energy is transported away into the substrate. On the other hand PMMA and PS have comparable $\Delta T_{i}$ and $d_{a p p}$ as was expected from their thermal properties. In addition, PS and PMMA have a more gradual increase in $\Delta T_{i}$ for decreasing film thicknesses above $d_{\text {app }}$. Taking into account the range of thermal transport and the simple 1D steady state model as described in Chapter 3 Figure 3.10, the range of $d_{a p p}$ is in the right order of magnitude.

\subsubsection{Monodisperse $\mathbf{P S}_{20}$ and $\mathbf{P S}_{650}$ films on silicon substrates}

In order to investigate the effect of polydispersity as well as molar mass on $\Delta T_{i}$ in more detail, two monodisperse PS polymers (PDI 1.03) with molar mass values $\left(M_{w}\right)$ of $20 \mathrm{~kg} \mathrm{~mol}^{-1}$ and $650 \mathrm{~kg} \mathrm{~mol}^{-1}$ were used as model compounds in this section. These polymers are referred to as $\mathrm{PS}_{20}$ and $\mathrm{PS}_{650}$, respectively. Furthermore, these model films allowed us to compare our results with existing literature reporting on PS film thickness dependent $T_{s}$ measurements.

A

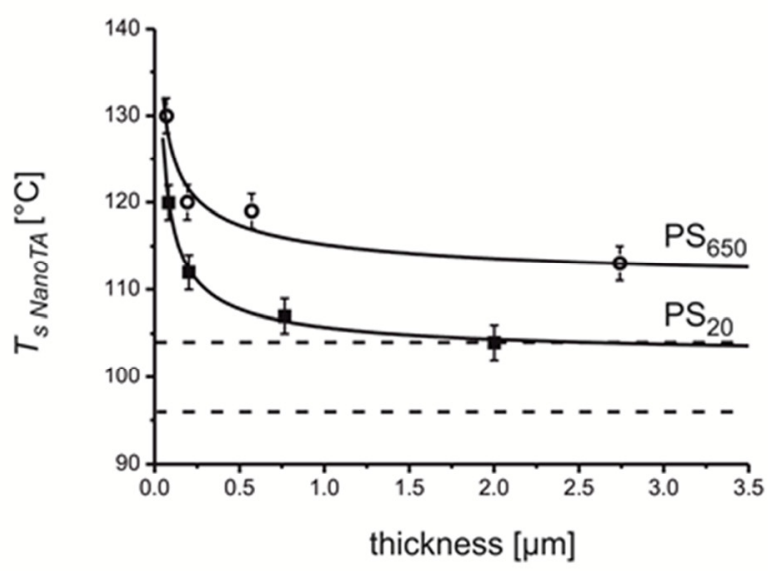

B

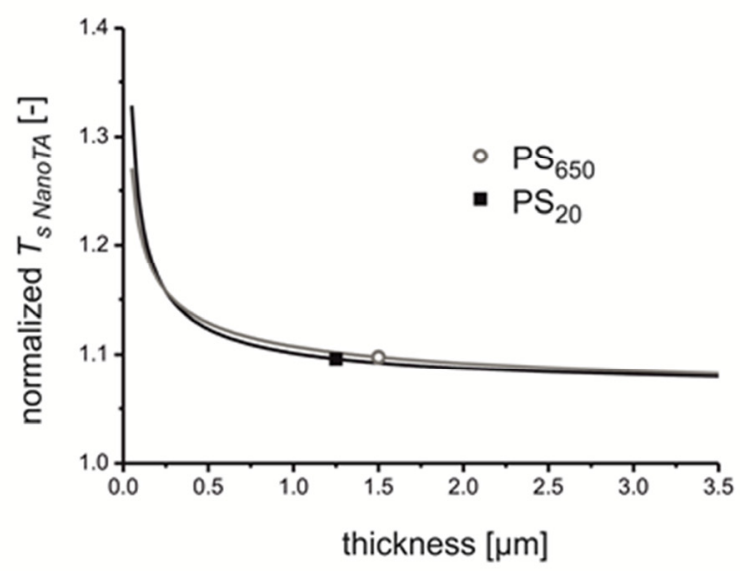

Figure 4.7: Measured $T_{s \text { NanoTA }}$ as a function of the film thickness on silicon for $P S_{20}$ and $P S_{650}$ films (A). The horizontal dashed lines represent the bulk $T_{g}$ for $P S_{20}$ (lower) and $P S_{650}$ (upper) as measured with DSC, respectively. The solid lines represent fits to the data according to equation 1. In (B) the normalized $T_{s \text { NanoTA }}$ as a function of the film thickness for both $P S_{650}$ as well as $P S_{20}$ on silicon supports is shown.

Figure 4.7A shows the thickness dependent surface $T_{S \text { NanoTA }}$ for $\mathrm{PS}_{20}$ and $\mathrm{PS}_{650}$ films on silicon. In analogy with the polydisperse polymers the measured $T_{s \text { NanoTA }}$ for the monodisperse PS samples increases for decreasing film thickness. In addition, the 


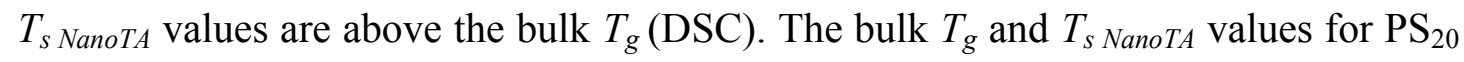
are lower compared to those for $\mathrm{PS}_{650}$ with comparable film thickness values. For the $T_{g}$ (bulk) this is in agreement with the higher contribution of chain ends to the free volume in polymer samples with a lower molar mass, reducing the $T_{g}{ }^{40,50}$ Apparently, this effect is (partly) taken into account in the measured $T_{S}$ NanoTA. The normalized

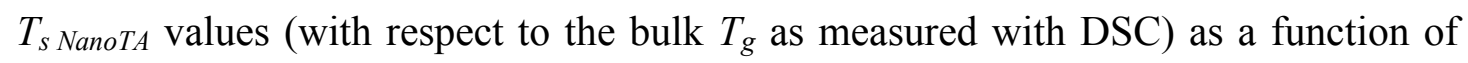
the film thickness for $\mathrm{PS}_{20}$ and $\mathrm{PS}_{650}$ (Figure 4.7B) show no significant differences.

Figure 4.8 shows the film thickness dependent $T_{s n T M A}$ for $\mathrm{PS}_{20}$ and $\mathrm{PS}_{650}$ films on silicon. The surface $T_{S} n T M A$ for $\mathrm{PS}_{20}$ is lower compared to $\mathrm{PS}_{650}$ with comparable film thicknesses. This was expected from the explanation described earlier for the

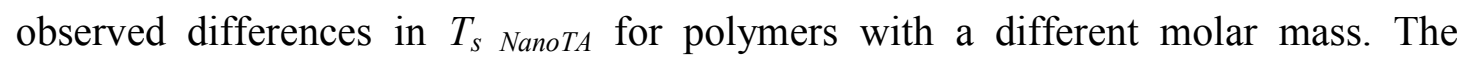
observed trend, i.e. decreasing $T_{S}{ }_{n T M A}$ for decreasing films thickness, is in agreement with previously described literature. ${ }^{26}$

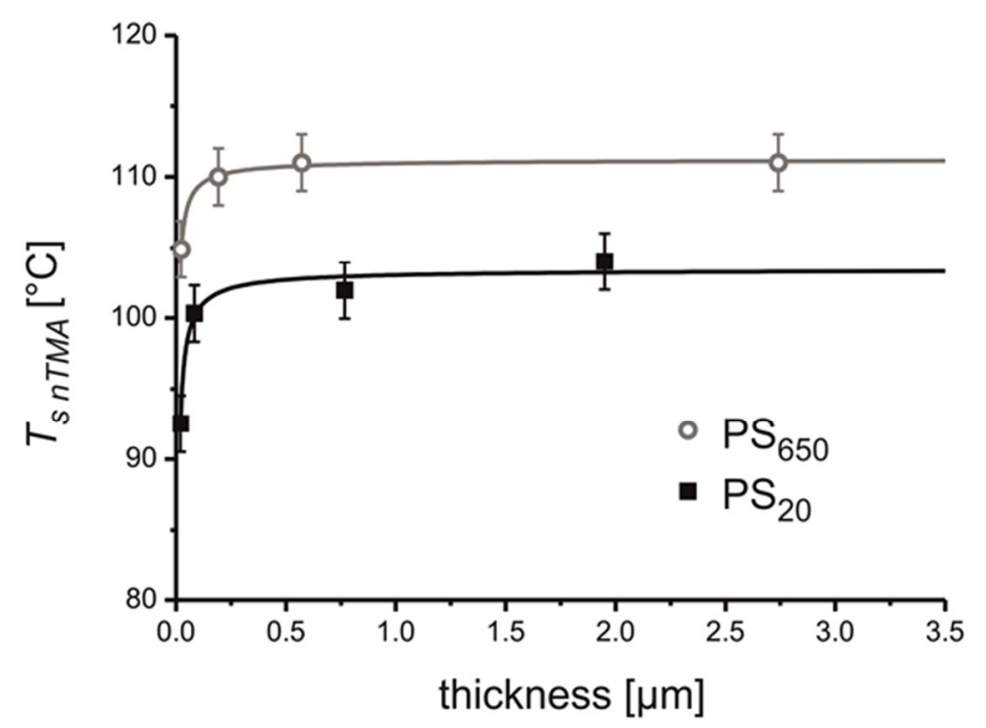

Figure 4.8: $T_{\text {s nTMA }}$ as a function of the film thickness on silicon for $P S_{20}$ and $P S_{650}$. The solid lines represent fits to the data according to equation 1.

The measured $T_{S \text { NAnoTA }}$ and $T_{S}$ nTMA for the monodisperse PS films were used to calculate $\Delta T_{i}$ according to equation 2 as described earlier. Figure 4.9 shows the calculated $\Delta T_{i}$ as a function of the film thickness. It is obvious that the calculated $\Delta T_{i}$ for $\mathrm{PS}_{20}$ and $\mathrm{PS}_{650}$ completely overlap. Despite the measured absolute differences in $T_{s}$, the thermal properties ( $\lambda$ and $C_{p}$ ) of the $\mathrm{PS}_{20}$ and $\mathrm{PS}_{650}$ samples were expected to be of comparable values, which explains the observed overlap. The overlap in $\Delta T_{i}$ for similar film thicknesses justifies the assumption that heat flow to good thermally conductive substrates dominates the over prediction of the measured $T_{s \text { app }}$ of polymer samples. The $d_{a p p}$ was calculated to be $515 \pm 67 \mathrm{~nm}$ and $590 \pm 77 \mathrm{~nm}$ for $\mathrm{PS}_{20}$ and 
$\mathrm{PS}_{650}$, respectively. These numbers are, although slightly lower still in good agreement with the $d_{\text {app }}$ reported for the PS sample with a PDI of $\sim 3.4$.

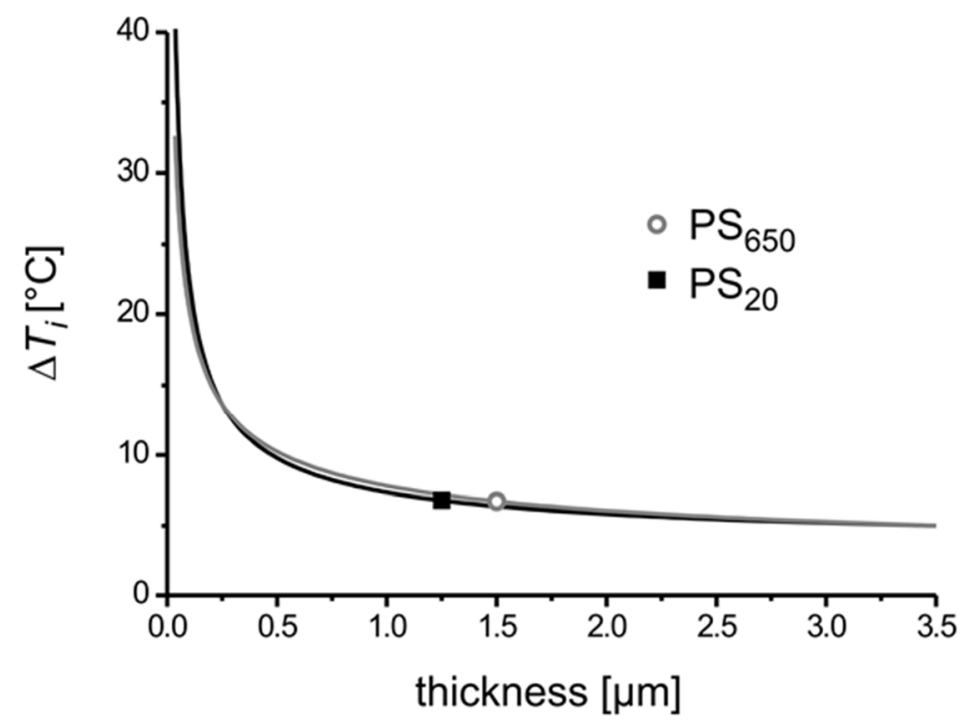

Figure 4.9: The calculated deviation in interface temperature according to equation 2 $\left(x=5^{\circ} \mathrm{C}\right)$ as a function of the film thickness on silicon for $P S_{20}$ and $P S_{650}$.

For a $50 \mathrm{~nm}$ thick $\mathrm{PS}_{20}$ or $\mathrm{PS}_{650}$ film on silicon the expected deviation in $T_{i}$ is approximately $34 \pm 6{ }^{\circ} \mathrm{C}$ and $29 \pm 5{ }^{\circ} \mathrm{C}$, respectively. This amount of deviation from the actual polymer thermal transition temperature is significant. Despite the observed deviation in $T_{i}$ we have seen that NanoTA is still capable of distinguishing between materials with small differences in absolute transition temperatures, although it resulted in the measurement of apparent transition temperatures for film thicknesses below $d_{\text {app }}$. In addition, Soles and coworkers ${ }^{43}$ reasoned that for thinner films the amount of tip penetration for the heated probe during NanoTA is less compared to penetration of very thick films. This results in a lower contact area at the probe tip sample interface. The increased heat flow into the substrate through a decreased contact area results in an under prediction of the calibrated $T_{i}$. Since this is incorporated within our measurements we can not clearly separate these effects. Based on the measured over prediction of $T_{i}$ we can at least conclude that heat dissipation into the solid supports dominates $\Delta T_{i}$.

Soles and coworkers ${ }^{43}$ reported a value of $\sim 129^{\circ} \mathrm{C}$ for the $T_{S \text { NanoTA }}$ for a $110 \mathrm{~nm}$ thick PS film $\left(M_{w} \sim 20 \mathrm{~kg} \mathrm{~mol}^{-1}\right)$ on $\mathrm{SiO}_{2}$. The temperature ramp rate used was $10^{\circ} \mathrm{C} \mathrm{s}^{-1}$. They reported on a bulk $T_{g}$ (DSC) value of $100{ }^{\circ} \mathrm{C}$ for this polymer. Based on our results the following corrections to their reported $T_{S \text { NanoTA }}$ value are proposed: i) $\Delta T_{i}$ for a $110 \mathrm{~nm}$ thick PS film on silicon is $\sim 21^{\circ} \mathrm{C}$ and $i$ ) the difference between $T_{s}$ and $T_{g}$ due to the mechanism of probing with NanoTA was estimated to be $\sim 10{ }^{\circ} \mathrm{C}$ 
for PS films on glass and silicon substrates. Subtraction of these values results in a corrected $T_{S}$ NanoTA value of $\sim 98{ }^{\circ} \mathrm{C}$. This is in close agreement with their reported bulk $T_{g}$ value. Figure 4.10 shows the original data for $T_{S \text { NanoTA }}$ values of PS films on silicon substrates reported by Soles and coworkers. ${ }^{43}$ Their reported data points were corrected following the same reasoning as described above for the $110 \mathrm{~nm}$ thick film.

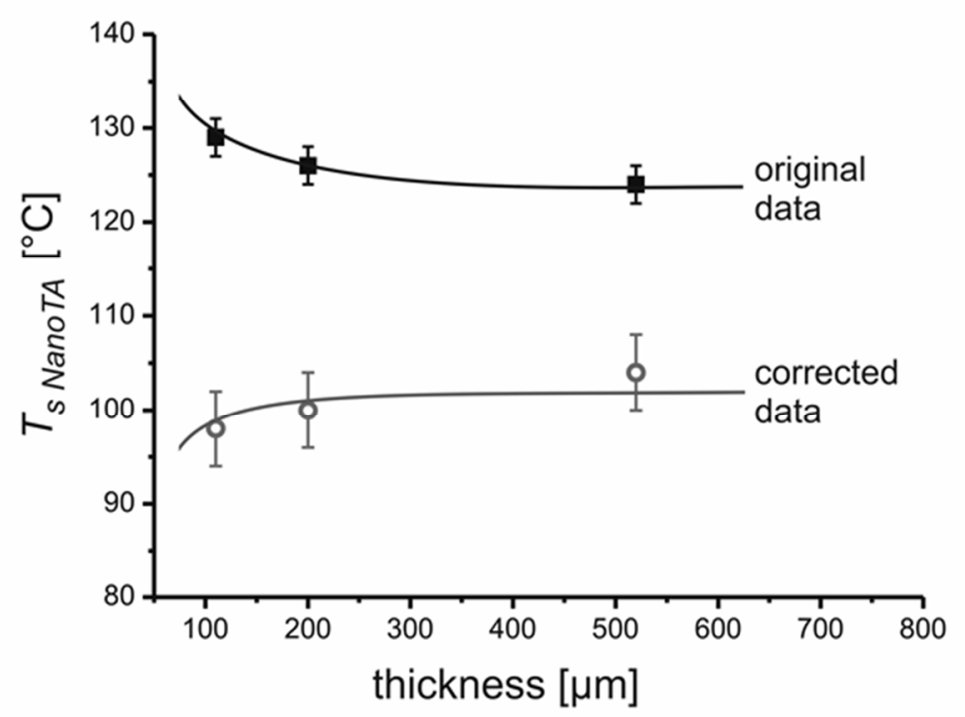

Figure 4.10: $T_{s}$ NanoTA data as a function of the PS film thickness on silicon substrates as reported by Soles and coworkers (filled black squares). ${ }^{43}$ The temperature ramp rate used was $10{ }^{\circ} \mathrm{C} \mathrm{s}^{-1}$. This data was corrected (open red circles) according to the measured $\Delta T_{i}$ (shown in Figure 4.9) as well as for the estimated $\sim 10{ }^{\circ} \mathrm{C}$ overestimation of the transition temperature measured with NanoTA compared to DSC bulk measurements.

The corrected $T_{S \text { NanoTA }}$ data shown in Figure 4.10 are in good agreement with the expected decrease in $T_{s}$ for thin PS films $(d<100 \mathrm{~nm})$. The presented calculation and data in Figure 4.10 validate our methodology for the estimation of $T_{s}$ for polymer thin films with film thicknesses below $d_{\text {app }}$.

The obtained insight in $\Delta T_{i}$ as a function of polymer film thicknesses is useful for the determination of surface transition temperatures. In addition, the measured $\Delta T_{i}$ can be of use for the estimation of $T_{i}$ during scanning thermal probe lithography as described in Chapters 7 and 8 of this Thesis. Future efforts should be directed towards the development of a universal calibration method based on polymer thermal transitions measurements, taking film thickness and substrate thermal conductivity into account. 


\subsection{Conclusions}

The expected deviation of the effective $T_{i}$ from the calibrated $T_{i}$ during NanoTA measurements of thin polymer films at good thermally conductive substrates was experimentally evaluated. Comparison of polymer film softening temperatures measured with NanoTA and nTMA showed that for $50 \mathrm{~nm}$ thick polymer films on $\mathrm{SiO}_{2}$ substrates the effective $T_{i}$ is reduced with tens of degrees Celsius compared to the calibrated $T_{i}$. The film thickness at which the temperature significantly starts to deviate from the surface $T_{s}$ of thick films $(d \geq 3.5 \mu \mathrm{m})$ when taking experimental differences into account was several hundreds of nanometers $\left(d_{a p p}\right)$ for PS, PMMA and $\mathrm{PC}$ films on $\mathrm{SiO}_{2}$ substrates. These results are important for the further development of NanoTA as an accurate nanotechnology tool. In addition these results are useful in other thermal scanning probe based approaches in which polymer films are employed and precise knowledge of the heated probe tip-sample interface temperature is required.

\subsection{Experimental}

Materials: PVC powder and PS granulate were obtained from Aldrich (Milwaukee, WI, USA). PMMA powder was purchased from Jansen chimica (Beerse, Belgium) and PC was a gift from Artecs B.V. (Enschede, The Netherlands). Monodisperse PS samples (PDI of 1.03) with molar mass values $\left(\mathrm{M}_{\mathrm{w}}\right)$ of $20 \mathrm{~kg} \mathrm{~mol}^{-1}$ and $650 \mathrm{~kg} \mathrm{~mol}^{-1}$ were obtained from Sigma-Aldrich (St. Louis, MO, USA). Butylated hydroxytoluene (BHT), stabilized tetrahydrofuran (THF) and toluene were purchased from Biosolve (Valkenswaard, The Netherlands). Chloroform and hydrogen peroxide 30\% were obtained from Merck (Darmstadt, Germany). Sulfuric acid 95-97\% was bought from Sigma-Aldrich (St. Louis, MO, USA). Acetone was received from Assink Chemie (Enschede, The Netherlands). Silicon wafers (CZ, type P, Boron, $<100>$, diameter $100 \mathrm{~mm}$ ) were obtained from Okmetic (Vantaa, Finland). Glass microscopy slides (1 $\mathrm{mm}$ thick) were bought from Menzel-Gläser (Braunschweig, Germany). Milli-Q water was produced by a Millipore Synergy system (Billireca, MA, USA). All chemicals were used as received.

Film preparation: Films were prepared by spin-coating polymer solutions (typically between $1 \mathrm{wt} \%$ and $15 \mathrm{wt} \%$ ) on pre-cut and pre-cleaned $15 \times 15 \mathrm{~mm}^{2}$ silicon or glass substrates at 1000 - $2000 \mathrm{rpm}$ for 1 minute. PMMA and PS were dissolved in toluene whereas PVC and PC were dissolved in THF and chloroform, respectively, prior to spin coating. Following spin-coating the films were left to dry and anneal in vacuum for 24 hours at a temperature of $125^{\circ} \mathrm{C}$ (PVC and PS), $145^{\circ} \mathrm{C}$ (PMMA) and $175^{\circ} \mathrm{C}$ (PC). These annealing temperatures are approximately $30{ }^{\circ} \mathrm{C}$ above the measured $T_{g}$ for the respective bulk samples by DSC. 
Film thickness measurements: Film thickness values were determined using a Dimension 3100 AFM equipped with a hybrid scanner and a NanoScope IVa controller (Veeco/Digital Instruments (DI), Santa Barbara, CA, USA) operated in tapping mode using commercially available silicon cantilevers (PointProbe ${ }^{\circledR}$ Plus silicon probes, PPP-NCH, Nanosensors, Neuchatel, Switzerland). By introducing a scratch through the film down to the substrate the film thickness was determined as the step height between the substrate and polymer film surface as was measured with AFM. The film thickness was measured at three randomly chosen positions along the scratch and the measured values were averaged. In addition, for silicon supported films null-ellipsometry in a polarizer-compensator-sample-analyzer mode (Multiscope, Optrel, Berlin, Germany) at a fixed wavelength and angle of incidence of $632.8 \mathrm{~nm}$ (He-Ne laser) and $70^{\circ}$, respectively was used to confirm the measured film thickness values. A bilayer model (polymer film plus substrate) for a flat film ${ }^{51}$ was used for the calculation of the film thickness from the obtained angles $\Psi$ and $\Delta$. The refractive indices of PVC, PS, PMMA and PC at the experimental wavelength were $1.478,1.587,1.490$ and 1.580 , respectively. ${ }^{52}$ For $\mathrm{Si}$ a refractive index value of 3.858 and an extinction coefficient value of -0.018 were used. ${ }^{53}$

Nanoscale thermal analysis: Heatable AFM probes (type AN-2, with $200 \mu \mathrm{m}$ long cantilevers, Anasys Instruments, Santa Barbara, CA, USA) mounted in a Dimension D3100 equipped with a hybrid scanner and a NanoScope IVa controller (Veeco/Digital Instruments (di), Santa Barbara, CA, USA) were used for the nanoscale thermal analysis of the polymer films. The probe temperature was controlled with a Nano-TA2 controller (Anasys Instruments, Santa Barbara, CA, USA). The temperature was ramped to values above the softening temperature at $10{ }^{\circ} \mathrm{C} \mathrm{s}^{-1}$. Prior to the experiments the probe temperature was calibrated using polymer standards with known melting points utilizing poly( $\varepsilon$-caprolactone) (PCL, $T_{m} \sim 55^{\circ} \mathrm{C}$ ), poly(ethylene) (PE, $T_{m} \sim 116^{\circ} \mathrm{C}$ ) and poly(ethylene terephthalate) (PET, $T_{m} \sim 235^{\circ} \mathrm{C}$ ).

Nanoscale thermal mechanical analysis: Isothermal NanoTA measurements were performed using a MultiMode AFM equipped with a NanoScope IV or V controller (Veeco/Digital Instruments (DI), Santa Barbara, CA, USA). A J scanner $(100 \times$ $100 \mu \mathrm{m}^{2} \mathrm{x}-\mathrm{y}$ scan size) was used and the temperature was controlled with a DI TAC high temperature accessory (Veeco/Digital Instruments (DI), Santa Barbara, CA, USA) heating the sample, as well as the cantilever. The cantilevers used were single beam Si contact mode probes (CONT-SPL Pointprobe, $k \sim 0.2 \mathrm{~N} \mathrm{~m}^{-1}, \mathrm{f}_{0} \sim 13 \mathrm{kHz}$, Nanoworld, Neuchatel, Switzerland) with a length and width of $450 \mu \mathrm{m}$ and $50 \mu \mathrm{m}$, respectively. At each temperature 25 force curves $(0.05 \mathrm{~Hz}$, ramp size $1 \mu \mathrm{m}$, trigger $+300 \mathrm{~nm})$ were collected in a $5 \times 5$ array $(x-y$ separation $1 \mu \mathrm{m})$ at the polymer surface. After each prescribed temperature increase the system was allowed to equilibrate for 
20 minutes. A thermocouple placed in contact with the thickest sample in close proximity of the cantilever showed that within 10 minutes the temperature reached the prescribed set value. The deflection sensitivity of the optical lever system was calibrated at every temperature on a bare silicon surface. The spring constants of the cantilevers used were determined with the thermal noise method. It was shown that the spring constants did not significantly change for the temperature range used throughout our experiments.

Differential scanning calorimetry (DSC): Bulk polymer samples of about 5 to $10 \mathrm{mg}$ in aluminum DSC pans were placed in a Perkin-Elmer Pyris1 DSC (Waltham, MA, USA). Heating and cooling traces, between $20^{\circ} \mathrm{C}$ and $180{ }^{\circ} \mathrm{C}$, of the amorphous polymer samples were recorded at $10{ }^{\circ} \mathrm{C} \mathrm{min}^{-1}$. The bulk $T_{g}$ values were determined as the extrapolated half $C_{p}$ temperature.

Gel permeation chromatography $(G P C)$ : The molar mass and PDI of the polymers were determined by GPC measurements in THF (PVC, PMMA and PS) or chloroform (PC) using a relative calibration method with PS standards. The GPC aperture was obtained from Hewlett Packard and belongs to the 1050 series. The separated samples were analyzed with a refractive index detector.

\subsection{References and notes}

1 Blümmel, J.; Perschmann, N.; Aydin, D.; Drinjakovic, J.; Surrey, T.; López-García, M.; Kessler, H.; Spatz, J. P. Biomaterials 2007, 28, 4739.

2 MacBeath, G.; Schreiber, S. L. Science 2000, 289, 1760.

3 Wells, M.; Crooks, R. M. J. Am. Chem. Soc. 1996, 118, 3988.

4 Huber, D. L.; Manginell, R. P.; Samara, M. A.; Kim, B. I.; Bunker, B. C. Science 2003, 301, 352.

5 Bertrand, P.; Jonas, A.; Laschewsky, A.; Legras, R. Macromol. Rapid. Commun.. 2000, $21,319$.

6 Mizutani, A.; Kikuchi, A.; Yamato, M.; Kanazawa, H.; Okano, T. Biomaterials 2008, 29, 2073.

7 Dimitrakopoulos, C. D.; Malenfant, P. R. L. Adv. Mater. 2002, 14, 99.

8 Hoppe, H.; Niggemann, M.; Winder, C.; Kraut, J.; Hiesgen, R.; Hinsch, A.; Meissner, D.; Sariciftci, N. S. Adv. Funct. Mater. 2004, 14, 1005.

9 Krebs, F. C. Sol. Energ. Mat. Sol. C 2009, 93, 394.

10 Jones, R. A. L. Curr. Opin. Colloid Interface Sci.. 1999, 4, 153.

11 Forrest, J. A.; Dalnoki-Veress, K. Adv. Colloid Interface Sci. 2001, 94, 167.

12 Fukao, K.; Miyamoto, Y. Phys. Rev. E 2000, 61, 1743.

13 Kawana, S.; Jones, R. A. L. Phys. Rev. E 2001, 63, 021501.

14 Keddie, J. L.; Jones, R. A. L.; Cory, R. A. Europhys. Lett. 1994, $27,59$.

15 Vallée, R. A. L.; Tomczak, N.; Kuipers, L.; Vancso, G. J.; van Hulst, N. F. Phys. Rev. Lett. 2003, $91,038301$.

16 Tomczak, N.; Vallée, R. A. L.; van Dijk, E. M. H. P.; Kuipers, L.; van Hulst, N. F.; Vancso, G. J. J. Am. Chem. Soc. 2004, 126, 4748. 
Priestley, R. D.; Ellison, C. J.; Broadbelt, L. J.; Torkelson, J. M. Science 2005, 309, 456.

García, R.; Pérez, R. Surf. Sci. Rep. 2002, 47, 197.

Giessibl, F. J. Rev. Mod. Phys. 2003, 75, 949.

Hafner, J. H.; Cheung, C. L.; Woolley, A. T.; Lieber, C. M. Prog. Biophys. Mol .Bio. 2001, 77, 73.

Mihalcea, C.; Scholz, W.; Werner, S.; Munster, S.; Oesterschulze, E.; Kassing, R. Appl. Phys. Lett. 1996, 68, 3531.

Engel, A.; Muller, D. J. Nat. Struct. Biol. 2000, 7, 715.

Ginger, D. S.; Zhang, H.; Mirkin, C. A. Angew. Chem. Int. Ed. 2004, 43, 30.

Liu, G. Y. Abstr. Pap. Am. Chem. Soc. 2000, 220, U431.

Wouters, D.; Schubert, U. S. Angew. Chem. Int. Ed. 2004, 43, 2480.

Bliznyuk, V. N.; Assender, H. E.; Briggs, G. A. D. Macromolecules 2002, 35, 6613.

Fryer, D. S.; Nealey, P. F.; de Pablo, J. J. Macromolecules 2000, 33, 6439.

Hammiche, A.; Pollock, H. M.; Song, M.; Hourston, D. J. Meas. Sci. Technol. 1996, 7, 142.

Nelson, B. A.; King, W. P. Rev. Sci. Instrum. 2007, 78, 023702.

Germinario, L. T.; Shang, P. P. J. Therm. Anal. Calorim. 2008, 93, 207.

King, W. P.; Kenny, T. W.; Goodson, K. E.; Cross, G.; Despont, M.; Dürig, U.; Rothuizen, H.; Binnig, G. K.; Vettiger, P. Appl. Phys. Lett. 2001, 78, 1300.

Lee, J.; Beechem, T.; Wright, T. L.; Nelson, B. A.; Graham, S.; King, W. P. J. Microelectromech. Syst. 2006, 15, 1644.

Mamin, H. J. Appl. Phys. Lett. 1996, 69, 433.

Vettiger, P.; Despont, M.; Drechsler, U.; Dürig, U.; Häberle, W.; Lutwyche, M. I.; Rothuizen, H. E.; Stutz, R.; Widmer, R.; Binnig, G. K. IBM J. Res. Dev. 2000, 44, 323.

Gotsmann, B.; Knoll, A. W.; Pratt, R.; Frommer, J.; Hedrick, J. L.; Dürig, U. Adv. Funct. Mater. 2010, 20, 1276.

Pires, D.; Hedrick, J. L.; De Silva, A.; Frommer, J.; Gotsmann, B.; Wolf, H.; Despont, M.; Dürig, U.; Knoll, A. W. Science 2010, 328, 732.

37 Szoszkiewicz, R.; Okada, T.; Jones, S. C.; Li, T. D.; King, W. P.; Marder, S. R.; Riedo, E. Nano Lett. 2007, 7, 1064.

Duvigneau, J.; Schönherr, H.; Vancso, G. J. Langmuir 2008, 24, 10825.

9 Wang, D. B.; Kodali, V. K.; Underwood, W. D.; Jarvholm, J. E.; Okada, T.; Jones, S. C.; Rumi, M.; Dai, Z. T.; King, W. P.; Marder, S. R.; Curtis, J. E.; Riedo, E. Adv. Funct. Mater. 2009, 19, 3696.

Gedde, U. W., Polymer Physics, 1st. ed., Kluwer Academic Publishers, Dordrecht, 1995.

A Hertzian contact model was assumed. The tip radius of curvature was estimated to be $30 \mathrm{~nm}$. Nelson, B. A.; King, W. P. Sens. Actuators A 2007, 140, 51.

Zhou, J.; Berry, B.; Douglas, J. F.; Karim, A.; Snyder, C. R.; Soles, C. Nanotechnology 2008, $19,495703$.

Schönherr, H.; Bailey, L. E.; Frank, C. W. Langmuir 2002, 18, 490.

Mark, J. E., Physical Properties of Polymers Handbook, 2nd ed., Springer Science, New York, 2007.

6 Shackelford, J. F.; Alexander, W., Materials Science and Engineering Handbook, 3rd ed., CRC press LLC, USA, 2001.

47 Hull, R., Properties of Crystalline Silicon, INSPEC, the Institution of Electrical Engineers, London, 1999. 
48 We note that for some polymers the surface $T_{s}$ was reported to increase with decreasing film thickness. It is expected that the error introduced in $T_{i}$ due to the close proximity of a good thermally conductive substrate is not affected by the sign of the $T_{s}$ change with decreasing film thickness.

49 Kaliappan, S. K.; Cappella, B. Polymer 2005, 46, 11416.

50 Fox, T. G.; Flory, P. J. J. Appl. Phys. 1950, 21, 581.

51 Santonicola, M. G.; de Groot, G. W.; Memesa, M.; Meszyńska, A.; Vancso, G. J. Langmuir 2010, 26, 17513.

52 a) For PVC: Barnes, M. D.; Kung, C. Y.; Lermer, N.; Fukui, K.; Sumpter, B. G.; Noid, D. W.; Otaigbe, J. U. Opt. Lett. 1999, 24, 121. b) For PMMA, PS and PC: Nikolov, I. D.; Ivanov, C. D. Appl. Optics 2000, 39, 2067.

53 Beinhoff, M.; Frommer, J.; Carter, K. R. Chem. Mater. 2006, 18, 3425. 



\section{Chapter 5}

\section{Surface Wetting Behavior of Poly(isoprene)-block- poly(ferrocenyldimethylsilane) Crystals During Heating and Melting Unveiled by AFM and NanoTA}

Crystallization of poly(ferrocenyldimethylsilane) (PFS) containing block copolymers in block-selective solvents constitutes an exciting driving force for the assembly of such macromolecules, allowing one to fabricate well-defined nanostructures with a semi-crystalline organometallic core. Recent studies have significantly contributed to the understanding of the assembly process. Depending on the block ratio, spherical or cylindrical micelles can be produced. The latter structures are of significant interest as nanowires. For many applications, nanowires are supported by solid substrates. To date, information on the interaction between nanostructures, formed by crystallization processes, and supporting substrates remains scarce. In this chapter the temperature dependent morphology of lamellar poly(isoprene)-block-poly(ferrocenyldimethylsilane) $\left(\mathrm{PI}_{76}-b\right.$ - $\left.\mathrm{PFS}_{76}\right)$ crystals deposited at the native oxide surface of silicon $\left(\mathrm{SiO}_{2}\right)$ or at highly ordered pyrolytic graphite (HOPG) is described. The effect of surface wettability of both blocks on these substrates is unveiled. Differential scanning calorimetry (DSC) was used to study the melting behavior of isothermally crystallized material. The onset of melting was observed at approximately $115^{\circ} \mathrm{C}$ followed by recrystallization processes resulting in a second melting endotherm at approximately $134{ }^{\circ} \mathrm{C}$. Preparation of the crystals in PI selective solvents resulted in a crystallization driven assembly of a semi-crystalline PFS core surrounded by a solvent swollen PI corona. $\mathrm{PI}_{76}-b$-PFS $\mathrm{S}_{76}$ crystals deposited on either $\mathrm{SiO}_{2}$ or HOPG had similar dimensions in terms of height $(\sim 13 \mathrm{~nm})$, width $(\sim 130 \mathrm{~nm})$ and length $(\sim 820 \mathrm{~nm})$. X-ray photoelectron spectroscopy (XPS) of $\mathrm{PI}_{76}-b$-PFS 76 crystals deposited on $\mathrm{SiO}_{2}$ before and after oxygen plasma etching confirmed the presence of a PI corona around a PFS core. The apparent melting temperature $\left(T_{m}\right)$ of the semi-crystalline PFS core was determined by nanoscale thermal analysis (NanoTA) to be $122{ }^{\circ} \mathrm{C} \pm 10{ }^{\circ} \mathrm{C}$. In situ examination of $\mathrm{PI}_{76}-b$-PFS 76 crystals deposited on $\mathrm{SiO}_{2}$ or $\mathrm{HOPG}$ with temperature controlled atomic force microscopy (AFM) unveiled morphology changes during heating and melting, which were ascribed to the hydrophilic and hydrophobic nature of the used substrates, respectively. The differences in morphology changes observed during heating and melting of $\mathrm{PI}_{76}-b$ - $\mathrm{PFS}_{76}$ crystals deposited on $\mathrm{SiO}_{2}$ and $\mathrm{HOPG}$ emphasize the role of interfacial energy between polymer based nanostructures and their supporting substrates. 


\subsection{Introduction}

The self assembly of amphiphilic block copolymers (BCP) in block selective solvents to form well defined nanometer sized aggregates with distinctive morphology has attracted extensive interest in recent years. In particular, the wide diversity available in architecture shape, size and composition makes them attractive for utilization in a broad range of possible areas of application, e.g. drug delivery, ${ }^{1-4}$ catalysis $^{5-8}$ and nanolithography. ${ }^{9-11}$ For instance, Dalhaimer and coworkers described the potential application of highly stable pegylated "worm-like" micelles with extended blood circulation times compared to their spherical counterparts, as drug carriers. ${ }^{12}$ Aydin and coworkers have reported on the block copolymer micelle nanolithography in combination with conventional nanolithography (i.e. photolithography and directed beam) approaches, to pattern 6-7 nm diameter gold particles with tunable inter-particle distances between 55 and $142 \mathrm{~nm}$ on silicon and glass substrates on different length scales. The patterned gold particles were functionalized with histidine tagged proteins in subsequent processing steps. ${ }^{13}$ Advantages of BCP self assembly nanolithography compared to more conventional lithography approaches include the high spatial resolution, achievable at high throughputs and low costs. $^{14-19}$ The functionality or properties of both corona and core of the self assembled BCP structures and devices can readily be adjusted by the alteration of one or more polymer building blocks. Poly(ferrocenyldimethylsilane) (PFS) based BCPs assemblies prepared in a selective solvent for the non-PFS block, reported in literature comprise an interesting class of architectures (see Figure 5.1). ${ }^{20-23}$ Due to the presence of both $\mathrm{Fe}(\mathrm{II})$ and $\mathrm{Si}$ in the organometallic polymer main chain, PFS possesses appealing properties in terms of redox activity ${ }^{24-26}$ and etch resistance. ${ }^{27,28}$ Furthermore PFS containing BCPs have been reported to be suitable precursors for ferromagnetic ceramics. ${ }^{29-31}$ For example, poly(dimethylsiloxane)- $b$-PFS, poly(methylvinylsiloxane)- $b$-PFS, PI- $b$-PFS, poly(styrene)- $b$-PFS and poly(methyl methacrylate)- $b$-PFS were prepared via controlled polymerization processes. ${ }^{32-34}$ Most of these yield rod-like cylindrical micelles in solution. Manners and coworkers have provided evidence that crystallization of the PFS core forming block is the driving force for the formation of the cylindrical architectures. ${ }^{35,36}$ The semi-crystallinity of the PFS rich core was proven with wide angle X-ray scattering (WAXS) measurements in which peaks with a $d$ spacing of $\sim 6.4 \AA$ were typically observed. The position of the peak was in good agreement with peak positions reported for PFS homopolymer films and PFS pentamer single crystals. ${ }^{37,38}$ The peaks observed were often weak and higher order peaks were mostly not detected. This indicates a most likely lower degree of crystallinity for the PFS core in the BCP self assembled architectures compared to typically observed for PFS homopolymers. Interestingly, results reported on by our group showed a rather dynamic PFS core for PMMA- $b$-PFS 
micelles prepared in acetone (i.e. selective solvent for PMMA) as was measured with temperature controlled ${ }^{1} \mathrm{H}-\mathrm{NMR}$ measurements. ${ }^{20}$ The absence of crystallinity in the PFS core for these micelles was independently confirmed by WAXS measurements which showed no Bragg peaks in the recorded spectra. These results pointed towards the solvophilicity driven micellar self-assembly for PMMA-b-PFS block copolymers in acetone.

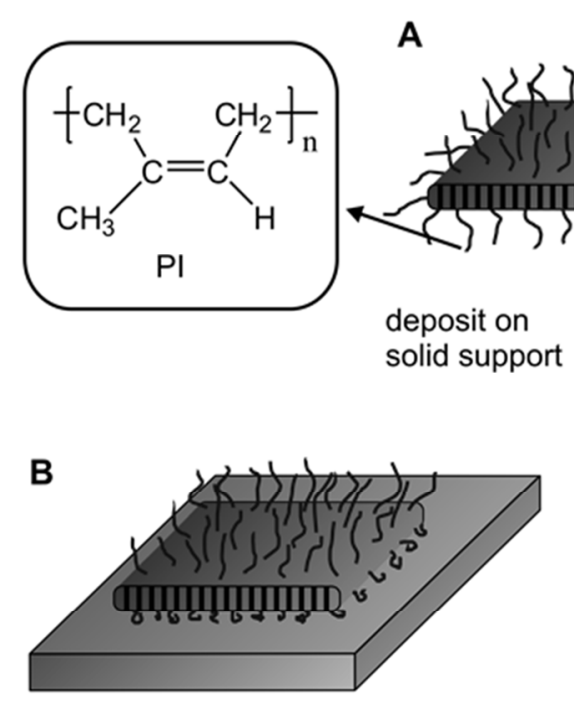

$\mathrm{SiO}_{2}$

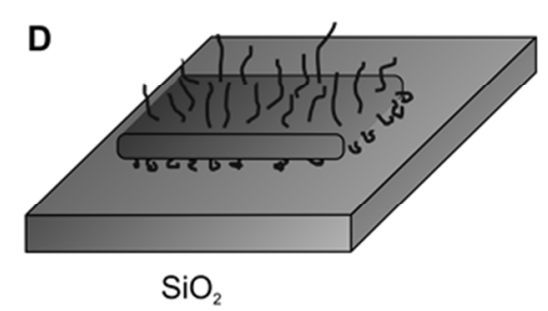

heat above $T_{m}$

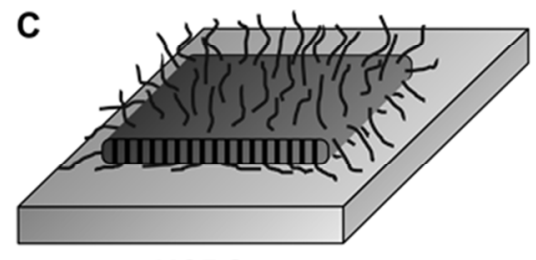

HOPG

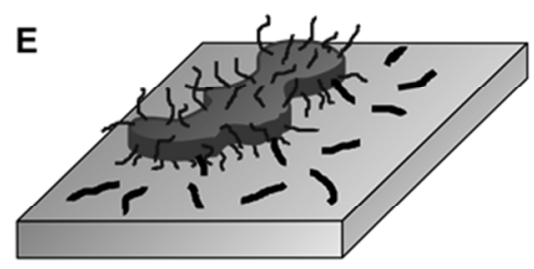

HOPG

Figure 5.1: Schematic representation of $\mathrm{Pl}_{76}-b-P F S_{76}$ platelet-like crystals in solution (A) before deposition on either $\mathrm{SiO}_{2}(B)$ or HOPG (C) via either spin coating or drop casting. The crystals adopt a platelet-like shape, which is related to the ratio of PI and PFS in the block copolymer, consisting of a crystalline PFS core surrounded by a corona of PI (hairy features). The chemical structures of PI and PFS are shown in the insets left and right of (A), respectively. The horizontal black grating represents the crystalline nature of the PFS core indicating chain packing order. Upon deposition on $\mathrm{SiO}_{2}$ or $\mathrm{HOPG}$ the crystal shape remains as before. Heating above $T_{m}$ causes the crystals on $\mathrm{SiO}_{2}$ to shrink in length (D) while on HOPG the crystal morphology falls apart and small micelle-like architectures (thick black lines) diffuse out of the remainder of the molten crystal $(E)$. 
While abundant literature is present reporting on multiple self assembled morphologies in different selective solvents and preparation conditions for different PFS based block copolymers, their utilization on surfaces remains scarce. Wang and coworkers $^{39}$ have reported on the cross-patterning of corona crosslinked PI- $b$-PFS cylindrical micelles on glass substrates via microfluidic patterning to eventually generate a 1D magnetic ceramic replica. Crosslinking of the corona provided additional strength to the cylindrical aggregates for further processing. In addition to the possible low stability of the formed PFS containing aggregates, the not yet fully developed knowledge of the behavior of these aggregates with respect to different substrates prevents their possible utilization as nanostructured devices.

This chapter describes the melting behavior of platelet-like $\mathrm{PI}_{76}-b$ - $\mathrm{PFS}_{76}$ micelles with a crystalline core deposited on either HOPG (hydrophobic) or $\mathrm{SiO}_{2}$ (slightly hydrophilic) substrates with NanoTA and temperature controlled AFM. These assemblies will be referred to as $\mathrm{PI}_{76}-b$ - $\mathrm{PFS}_{76}$ crystals. Comprehensive insight in thermally induced morphology changes is expected to provide further understanding in the molecular organization within $\mathrm{PI}_{76}-b$ - $\mathrm{PFS}_{76}$ platelet-like crystals and their thermal transitions. In addition, the introduction of NanoTA as an enabling nanotechnology tool for thermal analysis of nanometer sized architectures is discussed in detail taking the results described in Chapters 3 and 4 into account.

\subsection{Results and discussion}

\subsubsection{Differential scanning calorimetry of $\mathbf{P I}_{76}-b-\mathrm{PFS}_{76}$}

Crystallization and melting behavior of $\mathrm{PI}_{76}-b-\mathrm{PFS}_{76}$ bulk samples was first investigated with DSC. Figure 5.2A shows DSC heating thermograms of $\mathrm{PI}_{76}-b$ - $\mathrm{PFS}_{76}$ isothermally crystallized at temperatures $\left(T_{c}\right)$ between $85{ }^{\circ} \mathrm{C}$ to $95{ }^{\circ} \mathrm{C}$. The observed melting endotherms for $\mathrm{PI}_{76}-b$ - $\mathrm{PFS}_{76}$ are in good agreement with those reported on by Vancso and coworkers ${ }^{40,41}$ as well as by Rehahn and coworkers for PFS homopolymers. ${ }^{42}$ Two melting endotherms, indicated as type I and type II, were distinguished. Between the melting endotherms a weak crystallization exotherm is observed. The position of type II $\left(\sim 134{ }^{\circ} \mathrm{C}\right)$ is not affected by $T_{c}$, whereas type I shifts to higher temperatures for higher $T_{c}$ applied. Based on a comprehensive DSC and WAXS study for the PFS homopolymers ${ }^{40,41}$ it was concluded that type I melting is an irreversible transition ascribed to melting of the initial thin lamella formed at high degrees of super cooling followed by rapid recrystallization, resulting in the formation of thicker lamella with a higher melting transition temperature (type II). 
A

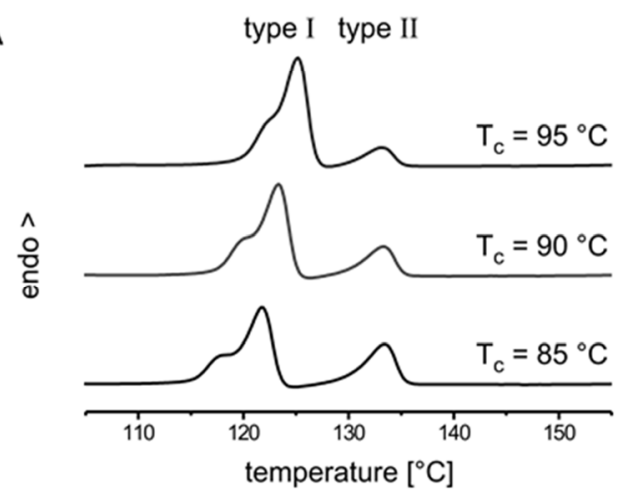

B

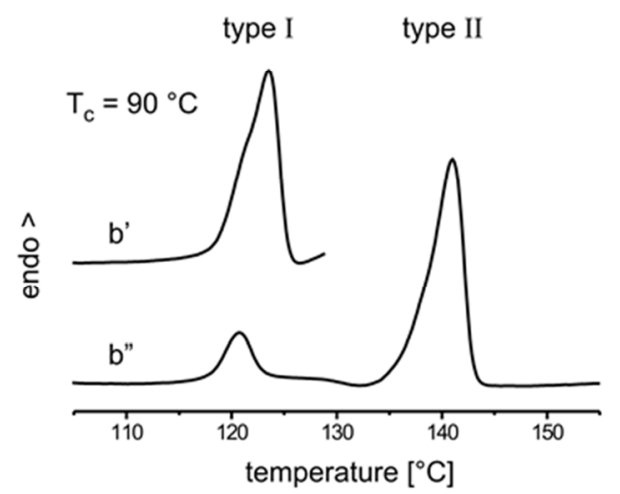

Figure 5.2: DSC thermograms of $\mathrm{Pl}_{76}-\mathrm{b}-\mathrm{PFS} \mathrm{S}_{76}$ showing the melting endotherms (A) for a sample crystallized at $85{ }^{\circ} \mathrm{C}, 90^{\circ} \mathrm{C}$ and $95{ }^{\circ} \mathrm{C}$, respectively (120 minutes). PFS melting occured within $115^{\circ} \mathrm{C}$ to $136{ }^{\circ} \mathrm{C}$. Type I lamella melting is for $\sim 76 \%$ irreversible $(B)$ as was observed for a sample heated to $129^{\circ} \mathrm{C}\left(b^{\prime}\right)$ followed by crystallization at $90{ }^{\circ} \mathrm{C}$ and heating to $150{ }^{\circ} \mathrm{C}\left(b^{\prime \prime}\right)$. The temperature was ramped at $10^{\circ} \mathrm{C} \mathrm{min}^{-1}$.

The observed rapid recrystallization was explained by the presence of a high number of nuclei still present up to the equilibrium melting temperature of PFS $\left(T_{m}{ }^{0} \sim 143^{\circ} \mathrm{C}\right) .{ }^{40,41}$

Figure 5.2B shows the DSC heating traces of a sample isothermally crystallized at $90{ }^{\circ} \mathrm{C}$ (120 minutes) initially up to $129^{\circ} \mathrm{C}$ (b', 15 minutes) subsequently cooled to $90{ }^{\circ} \mathrm{C}$ and after crystallization heated to $160{ }^{\circ} \mathrm{C}$ (b"). Obviously, melting of type I lamella in $\mathrm{PI}_{76}-b$ - $\mathrm{PFS}_{76}$ is not completely irreversible as is observed from the presence of a type I melting endotherm in the second heating trace. This is ascribed to the restricted chain mobility in the confined phase separated morphology, reducing the formation of thicker lamella. ${ }^{43}$ We note that, Rehahn and coworkers did not observe any recrystallization for PS- $b$-PFS. ${ }^{42}$ This was attributed to the much better defined lamellar thicknesses in the diblock copolymers, which is a well known phenomenon. ${ }^{43}$

Vancso and coworkers ${ }^{40}$ reported on the determination of the enthalpy of fusion $\left(\Delta H^{0}\right)$ for $100 \%$ crystalline PFS to be $\sim 35 \mathrm{~J} \mathrm{~g}^{-1}$ based on WAXS and DSC measurements. The recent value of $\sim 38 \mathrm{~J} \mathrm{~g}^{-1}$ for $\Delta H^{0}$ reported on by Rehahn and coworkers $^{42}$ is in good agreement with the earlier Vancso et al. value. Using these values for $\Delta H^{0}$ and the measured melting enthalpies (in $\mathrm{J} \mathrm{g}^{-1}$ ) for the type I lamella with DSC normalized to the PFS mass fraction $(\sim 78 \mathrm{wt} \%)$ in $\mathrm{PI}_{76}-b-\mathrm{PFS}_{76}$, the degree of crystallinity of isothermally crystallized samples at temperatures between $80{ }^{\circ} \mathrm{C}$ and $105{ }^{\circ} \mathrm{C}$ was calculated to be approximately $23 \%$ up to $37 \%$, respectively.

The equilibrium melting temperature $\left(T_{m}{ }^{0}\right)$ for PFS in $\mathrm{PI}_{76}-b-\mathrm{PFS}_{76}$ bulk samples was determined from a linear Hoffman-Weeks plot, ${ }^{44}$ as is shown in Figure 5.3. The 
melting temperatures were chosen as the peak values of the type I melting endotherms since these correspond to melting of the original crystals formed. The equilibrium melting temperature of the PFS block in $\mathrm{PI}_{76}-b-\mathrm{PFS}_{76}$ was determined from the intersection of the solid and dashed lines corresponding to $T_{m}=T_{c}=T_{m}{ }^{0}=\sim 144{ }^{\circ} \mathrm{C}$. This is in good agreement with the work of Vancso and coworkers ${ }^{40,41}$ for PFS homo polymers with similar degrees of polymerization.

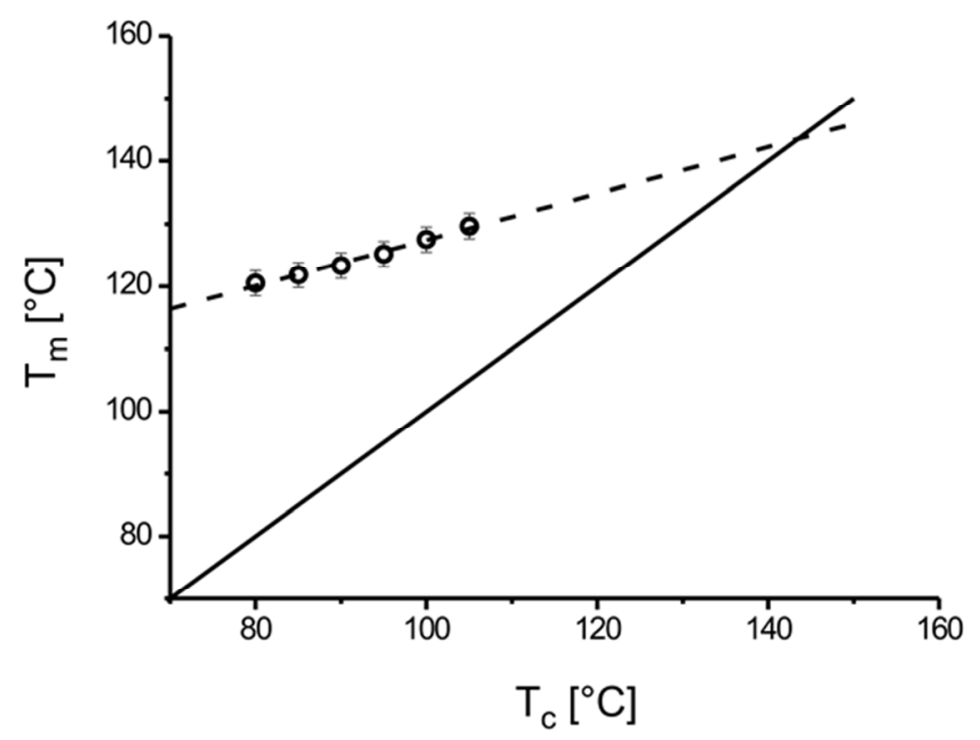

Figure 5.3: Hoffman-Weeks plot of $\mathrm{Pl}_{76}-b-P F S_{76}$ melting temperature vs. crystallization temperature. The crystallization temperatures were between $85{ }^{\circ} \mathrm{C}$ up to $105{ }^{\circ} \mathrm{C}$. The solid line represents the line $T_{c}=T_{m}$ and the dashed line is a linear least square fit to the data.

Rehahn and coworkers ${ }^{42}$ on the other hand argued that the Hoffman-Weeks analysis results in a $\mathrm{T}_{\mathrm{m}}{ }^{0}$ that is far too low due to the assumption that the thickness of the lamella is inversely proportional to the super-cooling $\Delta T$ (i.e. $T_{m}{ }^{0}-T_{c}$ ). Especially at higher $T_{c}$ they observed deviations from the assumed linearity. They calculated $T_{m}{ }^{0}$ for PFS based on the Gibbs-Thomson procedure, ${ }^{45}$ which takes deviations from the assumed linearity in the Hoffman-Weeks analysis into account, in PFS homopolymer and PS- $b$-PFS to be $215^{\circ} \mathrm{C}$ and $179{ }^{\circ} \mathrm{C}$, respectively. Despite this argumentation their reported Hoffman-Weeks analysis for $T_{c}$ values that were in the same range as the ones reported in this study, resulted in a $T_{m}{ }^{0}$ of $\sim 144{ }^{\circ} \mathrm{C}$ for PFS in both PFS homopolymer as well as in PS- $b$-PFS. 
A

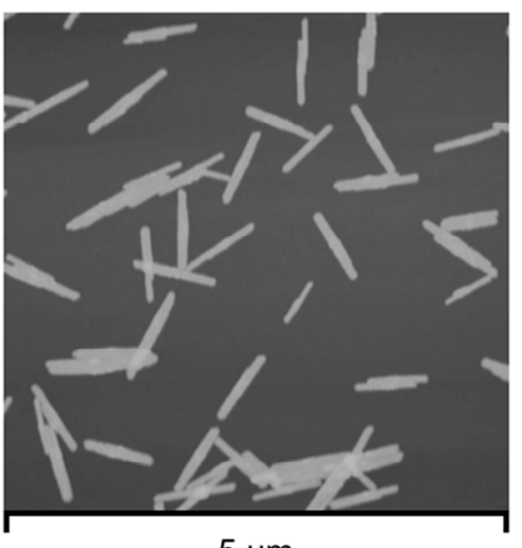

$5 \mu \mathrm{m}$

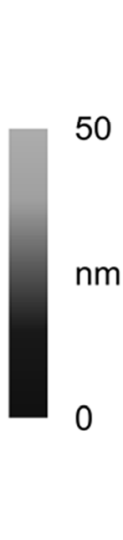

$\mathrm{B}$

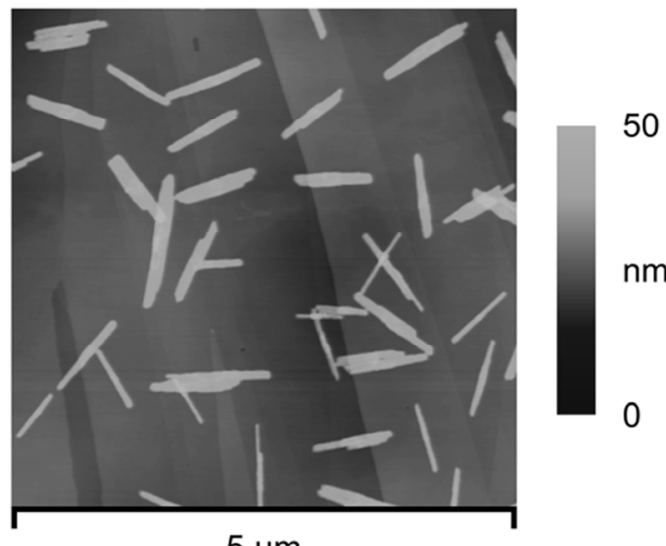

$5 \mu \mathrm{m}$

Figure 5.4: Tapping mode AFM height images of platelet-like $P_{76}-b-P F S_{76}$ crystals deposited on $\mathrm{SiO}_{2}(A)$ and $\mathrm{HOPG}(B)$.

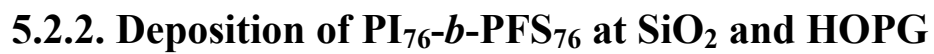

Figure 5.4 shows tapping mode AFM height images of $\mathrm{PI}_{76}-b$-PFS ${ }_{76}$ platelet-like crystals after spin casting them at either $\mathrm{SiO}_{2}$ (5.4A) or at HOPG (5.4B). The width, height and length of the crystals as casted were measured for 20 crystals to be $135 \pm 18 \mathrm{~nm}, 12.4 \pm 1.1 \mathrm{~nm}$ and $832 \pm 89 \mathrm{~nm}$ on $\mathrm{SiO}_{2}$ and $128 \pm 28 \mathrm{~nm}$, $12.9 \pm 1.3 \mathrm{~nm}$ and $805 \pm 111 \mathrm{~nm}$ on HOPG, respectively, indicating similar dimensions. For crystals prepared in another batch the width and height were comparable, yet the length of these crystals was $\sim 4 \mu \mathrm{m}$. The high width to height ratios obtained indicate the presence of a core consisting of PFS chain folded lamellar domains surrounded by a corona of PI chains which seems to be independent of the crystallization process.

XPS spectroscopy was used to obtain information of the composition of the air exposed top layer of the $\mathrm{PI}_{76}-b$ - $\mathrm{PFS}_{76}$ crystals deposited at $\mathrm{SiO}_{2}$ substrates before and after oxygen reactive ion etching $\left(\mathrm{O}_{2}\right.$ - $\left.\mathrm{RIE}\right)$. The etch step was used to remove the organic material from the corona and the core of the micellar crystals in order to expose the iron present in the PFS rich core. Figure 5.5 shows high resolution XPS $\mathrm{Fe} 2 \mathrm{p}$ core spectra of $\mathrm{PI}_{76}-b$ - $\mathrm{PFS}_{76}$ platelet-like crystals after deposition on $\mathrm{SiO}_{2}$ substrates before and after oxygen reactive ion etching $\left(\mathrm{O}_{2}\right.$-RIE). Before plasma etching no Fe could be detected indicating that the PFS rich core is indeed fully covered by a PI corona of at least $7 \mathrm{~nm}$ thick (i.e. the estimated escape depth of the photo electrons). Following plasma etching, in which the organic material was removed, Fe $2 p$ bands at $712.7 \mathrm{eV}\left(2 \mathrm{p}_{3 / 2}\right)$ and $726.1 \mathrm{eV}\left(2 \mathrm{p}_{1 / 2}\right)$ were observed. This is ascribed to the presence of $\mathrm{Fe}$ in a higher oxidation state (after $\mathrm{O}_{2}$-RIE) compared to Fe in ferrocene. ${ }^{28}$ 

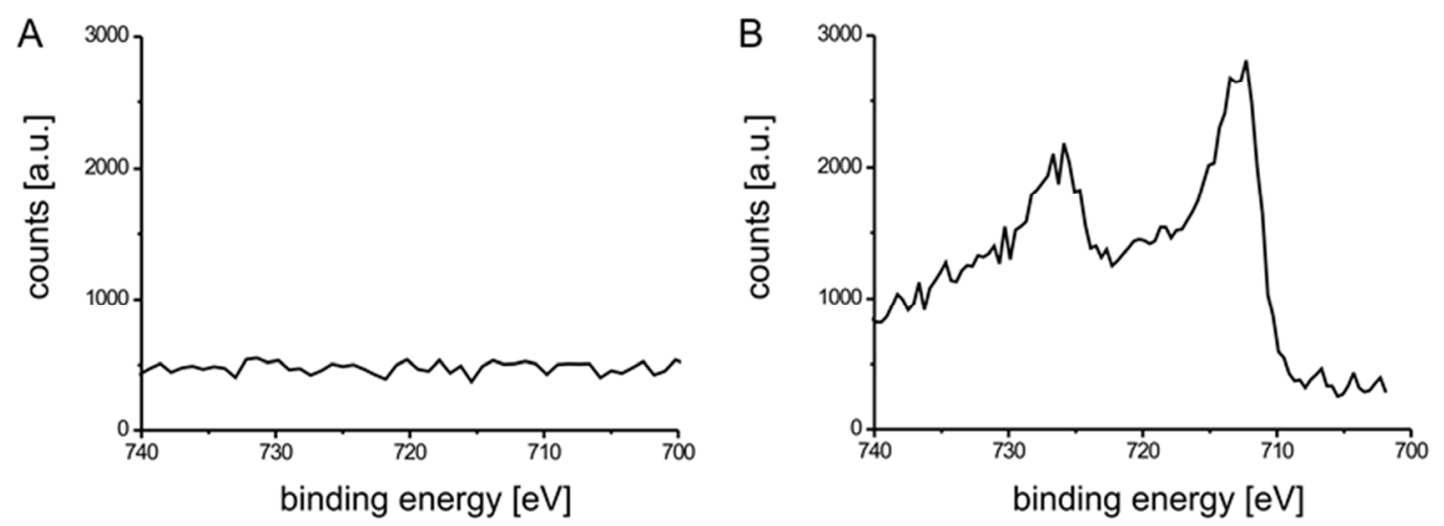

Figure 5.5: High resolution XPS Fe2p core spectra of $\mathrm{Pl}_{76}-b-P F S_{76}$ crystals deposited on silicon substrates before (A) and after $(B) \mathrm{O}_{2}-R I E$. Before etching no Fe could be detected while upon etching the Fe rich PFS core was revealed. This indicates that the PFS core is fully covered by a PI corona.

\subsubsection{Nanoscale thermal analysis of $P_{76}-b$-PFS ${ }_{76}$ crystals}

NanoTA was used to determine the melting transition temperature of $\mathrm{PI}_{76}-b$-PFS 76 platelet-like crystals deposited on $\mathrm{SiO}_{2}$ surfaces. NanoTA is an upcoming nanotechnology tool for spatially controlled thermal analysis of surfaces of materials such as soft matter. Typically the deflection of the cantilever is monitored as a function of the calibrated probe tip temperature $\left(T_{i}\right)$ in constant height mode (see Chapter 2). ${ }^{46}$ Upon increasing $T_{i}$ the sample in close proximity of the tip contact point is heated. Hence the sample will thermally expand, which is measured as an increase in cantilever deflection. Once the melting point of a sample is reached, the material softens and the tip starts to penetrate into the sample surface. This is monitored as a marked decrease in the deflection signal. Despite several attempts for individual $\mathrm{PI}_{76}-b$ - $\mathrm{PFS}_{76}$ platelet-like crystals no transition temperature was detected by NanoTA. This is explained by i) the crystal geometry i.e. that the crystals are very thin and as a consequence the small penetration of the heated probe tip into the crystal upon melting of the PFS core is not detectable and ii) the presence of silicon (good thermal conductor) close to the tip-sample interface cools down the probe tip significantly for NanoTA on these thin structures (see Chapter 3 ). ${ }^{47}$ Hence the probe tip temperature is assumed to significantly deviate from the previously calibrated "set" temperature. In other words, single $\mathrm{PI}_{76}-b-\mathrm{PFS}_{76}$ platelet-like crystals are below the physical limit of the technique.

Drop casting of $\mathrm{PI}_{76}-b-\mathrm{PFS}_{76}$ at $\mathrm{SiO}_{2}$ resulted in the formation of over micrometer thick aggregates of crystals piled up on top of each other. NanoTA runs on these aggregates were successful. Figure 5.6 shows a NanoTA graph of the crystals in which $T_{i}$ was ramped from $50{ }^{\circ} \mathrm{C}$ up to $200{ }^{\circ} \mathrm{C}$ at $10{ }^{\circ} \mathrm{C} \mathrm{s}^{-1}$. Since the PI corona 
continues to expand even upon melting of PFS, the marked drop typically observed for crystalline homopolymers is absent. Instead a lower overall thermal expansion is observed. The apparent melting point $\left(T_{m}\right.$ app $)$ is now defined as the intersect of the slopes at which both PI and PFS thermally expand and the horizontal plateau in which thermal expansion (PI) and sample penetration (molten PFS) are balanced. A value of $122 \pm 10^{\circ} \mathrm{C}$ was obtained for $T_{m \text { app }}$. The relatively high error value is ascribed to the use of polymer melting point standard materials for the probe tip temperature calibration (see Chapter 2).

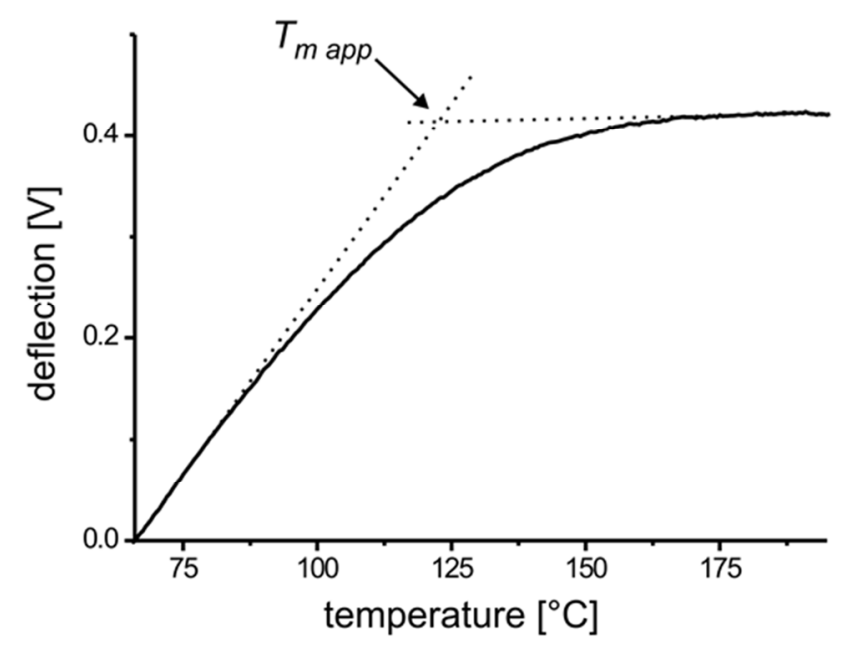

Figure 5.6: NanoTA graph of $\mathrm{Pl}_{76}-b-P F S_{76}$ crystals drop casted on silicon. The apparent melting point was calculated to be $122 \pm 10{ }^{\circ} \mathrm{C}$ (intercept of the two dotted lines). The temperature was ramped at $10^{\circ} \mathrm{C} \mathrm{s}^{-1}$.

The calculated $T_{m \text { app }}$ value of the semi-crystalline PFS core is in good agreement with the melting temperature range of PFS in bulk $\mathrm{PI}_{76}-b$ - $\mathrm{PFS}_{76}$ samples measured with DSC. We note that NanoTA uses typically high temperature ramp rates $\left(\sim 10^{\circ} \mathrm{C} \mathrm{s}^{-1}\right)$. Hence in close proximity of the tip-sample contact interface the sample is not in equilibrium at elevated temperatures. Therefore recrystallization as observed with DSC (typically $\sim 10{ }^{\circ} \mathrm{C} \min ^{-1}$ ) was not observed. Lowering the temperature ramp rate for NanoTA to below $\sim 3{ }^{\circ} \mathrm{C} \mathrm{s}^{-1}$ typically results in noisy NanoTA graphs.

\subsubsection{Isothermal AFM of $\mathrm{PI}_{76}-b$-PFS 76 at elevated temperatures}

Figure 5.7 shows temperature controlled tapping mode AFM height images of $\mathrm{PI}_{76}-b$ - $\mathrm{PFS}_{76}$ platelet-like crystals deposited on $\mathrm{SiO}_{2}$. The images shown are subsequent images taken at room temperature, $80^{\circ} \mathrm{C}, 100{ }^{\circ} \mathrm{C}$ and $130{ }^{\circ} \mathrm{C}$ and are part of a series following a temperature ramp profile mentioned in the figure caption. The most obvious change observed in crystal dimensions is the decrease in crystal length with increasing temperature, starting from $80{ }^{\circ} \mathrm{C}$ and higher temperatures. This 
decrease in crystal length occurred during the first few minutes after a temperature rise. Heating to $130{ }^{\circ} \mathrm{C}$ typically resulted in a $\sim 17 \pm 3 \%$ decrease in length. Upon increasing the temperature $10{ }^{\circ} \mathrm{C}$ or more the AFM needs to be equilibrated for a few minutes to eliminate thermal drift. In addition to find back the same area takes a few minutes as well. First images were typically collected after 20 minutes following the increase in temperature. The poor temporal resolution prevented any kinetic information to be obtained for the decrease in crystal length. Prolonged heating (up to $\sim 3$ hours) at the set temperatures showed little to no further decrease in length. Upon cooling to room temperature we found that the decrease in length was irreversible. At temperatures close to and above the expected melting point (up to $160^{\circ} \mathrm{C}$ ) the crystals remained their platelet-like shape.
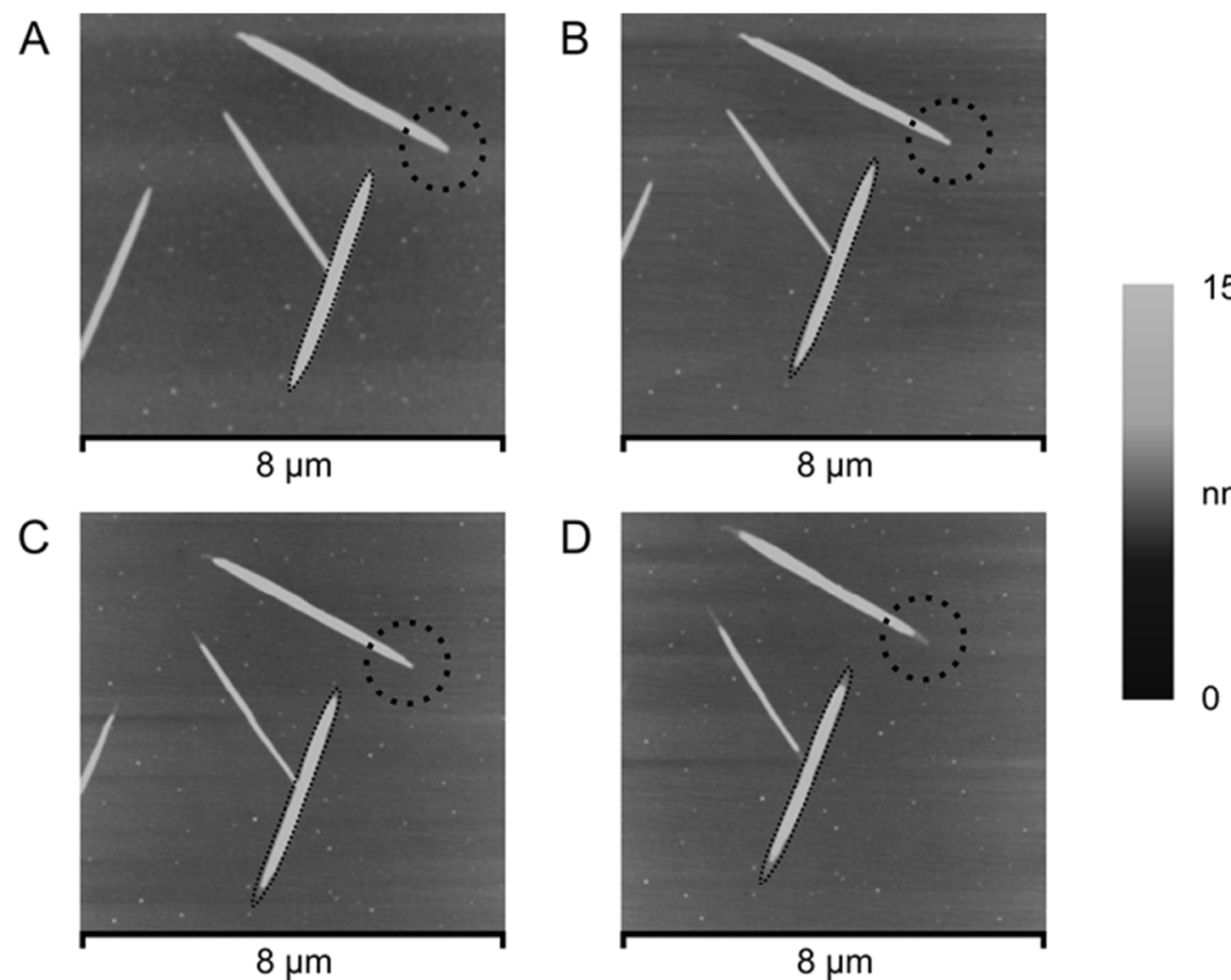

Figure 5.7: Temperature controlled tapping mode AFM images of platelet-like single $\mathrm{Pl}_{76}-b-P F S_{76}$ crystals on $\mathrm{SiO}_{2}$. The images shown here are initially at room temperature (A), 20 minutes at $80^{\circ} \mathrm{C}(B), 20$ minutes at $100^{\circ} \mathrm{C}(\mathrm{C})$ and 20 minutes at $130^{\circ} \mathrm{C}(\mathrm{D})$, respectively. The decrease in length upon increasing the temperature above $80^{\circ} \mathrm{C}$ is obvious when examining the crystal end located in the black dotted circle as well as from the overlay of the contour (thin dotted line) of one crystal at room temperature $(A)$ on the same crystal at higher temperatures $(B-D)$. Note: the $z$ scale was saturated for reasons of clarification. The followed temperature program started at room temperature followed by 80 minutes at $80^{\circ} \mathrm{C}$, 30 minutes at $100^{\circ} \mathrm{C}, 150$ minutes at $120^{\circ} \mathrm{C}$ and finally 130 minutes at $130^{\circ} \mathrm{C}$. 
In addition to $\mathrm{PI}_{76}-b$ - $\mathrm{PFS}_{76}$ crystals deposited on $\mathrm{SiO}_{2}$ surfaces (hydrophilic), the morphological changes of crystals deposited on HOPG surfaces (hydrophobic) during heating and melting were also unveiled with isothermal AFM measurements. Figure 5.8 shows temperature controlled tapping mode AFM phase images of $\mathrm{PI}_{76} b \mathrm{PFS}_{76}$ platelet-like crystals deposited on HOPG at temperatures of $80{ }^{\circ} \mathrm{C}$ up to $120{ }^{\circ} \mathrm{C}$ for different heating times. Interestingly the crystals show a slight decrease in length at temperatures above $100{ }^{\circ} \mathrm{C}$ (Figure 5.8C), with the formation of a very thin layer surrounding the crystals, which is visible in the AFM phase images. The thickness of this layer was estimated from the corresponding AFM height images to be below $1 \mathrm{~nm}$. We interpret the formation of this thin wetting layer as a result of the diffusion of PI- $b$-PFS molecules originating from the crystals on the HOPG surface. At $120^{\circ} \mathrm{C}$ the crystals show a typical molten morphology, which is in good agreement with the expected melting temperature being close to $120^{\circ} \mathrm{C}$.
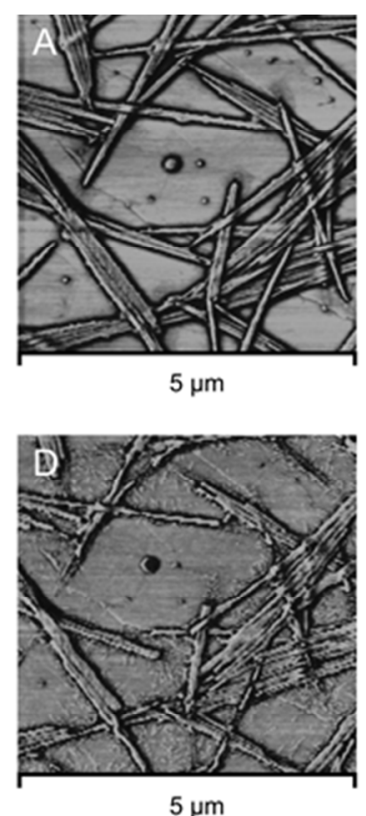

Figure 5.8: A series of AFM phase images of $P_{76}-b-P F S_{76}$ crystals on HOPG collected with temperature controlled tapping mode AFM at $(A)$ to $(B) 80^{\circ} \mathrm{C}$ (images collected at time 60 minutes and 85 minutes), (C) $100^{\circ} \mathrm{C}$ (formation of HOPG wetting layer can be observed), and $(D)$ to $(F) 120^{\circ} \mathrm{C}$ (images collected at different time interval of 30 minutes, 185 minutes and 490 minutes), respectively.

Control experiments with freshly cleaved HOPG surfaces without deposited crystals, following an identical temperature profile did not reveal the presence of contamination originating from the environment that could explain the presence of a wetting layer on HOPG at elevated temperatures. XPS of crystals deposited on $\mathrm{SiO}_{2}$ and HOPG surfaces before and after heating at $130{ }^{\circ} \mathrm{C}$ for 3 hours was used to 
evaluate whether iron that may originate from the PFS was exposed to the surface after melting. No iron was detected for crystals deposited at $\mathrm{SiO}_{2}$ surfaces before and after melting as well as after deposition at HOPG. For molten crystals deposited at HOPG Fe2p peaks were observed at $708.8 \mathrm{eV}\left(2 \mathrm{p}_{3 / 2}\right)$ and $721.5 \mathrm{eV}\left(2 \mathrm{p}_{1 / 2}\right)$ which is in agreement with previous values reported for PFS homopolymers. ${ }^{28}$ The Fe $2 p$ peak with a binding energy of $708.8 \mathrm{eV}$ is close to the value found for $\mathrm{Fe}$ in ferrocene. ${ }^{48}$ These results point toward the presence of PFS in the wetting layer on HOPG after heating of the crystals.

This remarkable difference in behavior of the crystals on $\mathrm{SiO}_{2}$ and HOPG upon increasing the temperature is explained in terms of substrate wettability of PI and PFS, respectively. Contact angle measurements of PI and quenched PFS melts on $\mathrm{SiO}_{2}$ and HOPG showed a preferred wetting (spreading) of PI on HOPG whereas on $\mathrm{SiO}_{2}$ no preferred wetting was observed. These measurements are in agreement with results reported on by Butt and coworkers ${ }^{49}$ and by Vancso and coworkers. ${ }^{50}$ Upon melting of the formed lamella the PI corona chains are no longer anchored at the $\mathrm{PI}_{76}-b$ - $\mathrm{PFS}_{76}$ interface and have an increased chain mobility. Hence PI starts to wet the HOPG surface which is observed as the diffusion of the small $\mathrm{PI}_{76}-b$-PFS 76 aggregates in Figure 5.8D-F.

In some rare cases for $\mathrm{PI}_{76}-b$ - $\mathrm{PFS}_{76}$ crystals deposited on $\mathrm{SiO}_{2}$, especially when two crystals were aligned on top of each other, thickening originating from the adjacent sites was observed for temperatures around $130{ }^{\circ} \mathrm{C}$. Figure 5.9 shows a temperature controlled tapping mode AFM height image as an example. The observed effect is ascribed to lamellar thickening. In analogy to DSC measurements of the bulk $\mathrm{PI}_{76}-b$-PFS ${ }_{76}$ material this might be explained by recrystallization of type I lamella at temperatures above $120{ }^{\circ} \mathrm{C}$ to form thicker type II lamella that have a higher melting point. In this particular case the adjacent site is expected to be a good nucleation point. After prolonged heating at increased temperatures (up to $160{ }^{\circ} \mathrm{C}$, images not shown) the well defined shape of the crystals remained. The thickened layer moved to one of the "arms" of the cross over crystals indicating that molecular rearrangements occur in the melt in order to minimize the free energy of the structure. 

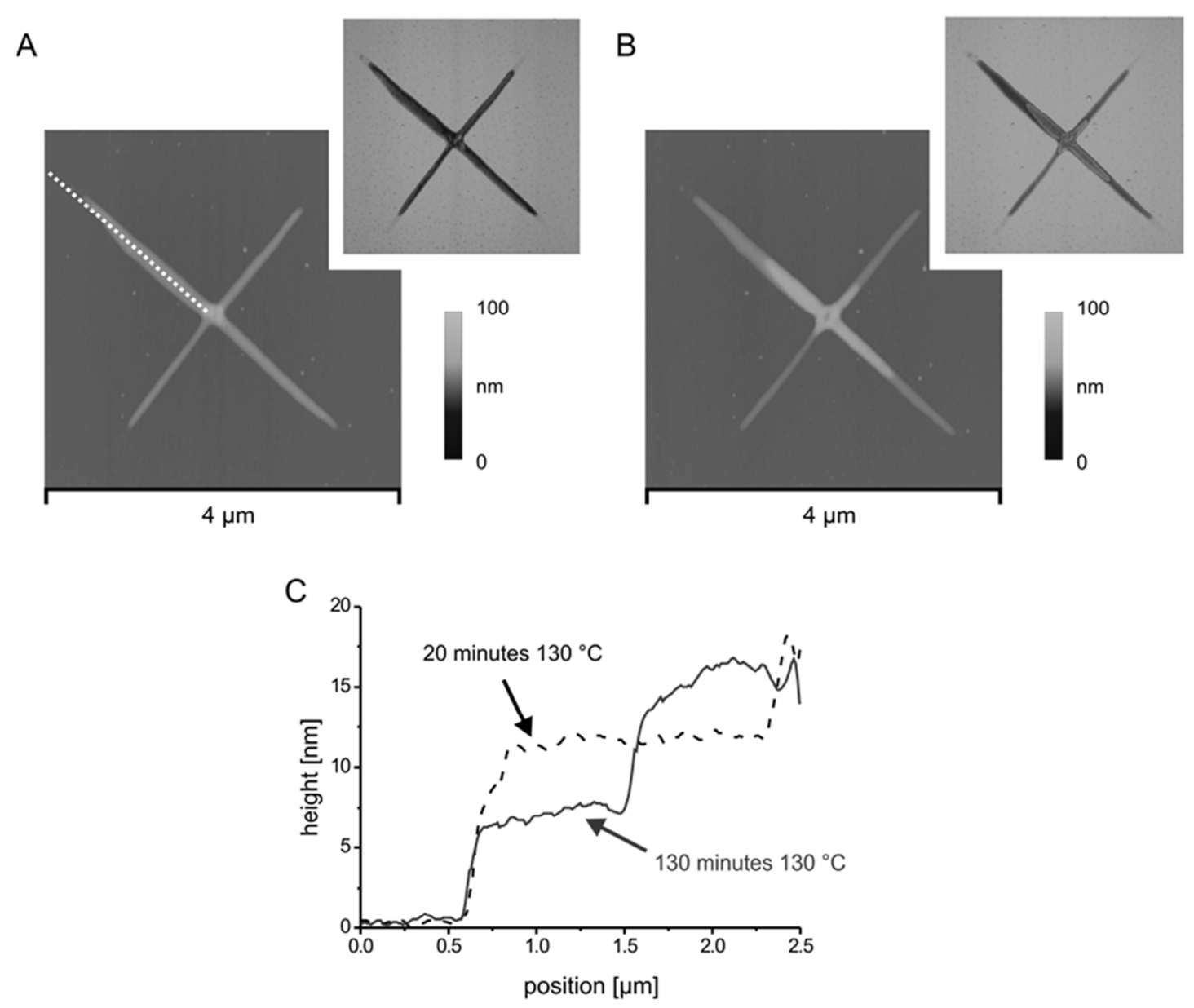

Figure 5.9: Tapping mode AFM height images of $\mathrm{Pl}_{76}-\mathrm{b}-\mathrm{PFS} \mathrm{S}_{76}$ crystals at $130{ }^{\circ} \mathrm{C}$ for 20 minutes $(A)$ and $130^{\circ} \mathrm{C}$ for 130 minutes $(B)$ showing the growth of a thickened layer upon prolonged heating at $130^{\circ} \mathrm{C}$ (pointed at by the white arrows in $B$ ). The insets in $A$ and $B$ are tapping mode phase images ( $z$ scale $120^{\circ}$ ) of the same area shown in the height images. The corresponding cross-sections along the crystal longitudinal axis represented by the white dotted line in $A$ are shown in (C).

Despite the interesting phenomena observed upon heating $\mathrm{PI}_{76}-b$ - $\mathrm{PFS}_{76}$ crystals on $\mathrm{SiO}_{2}$ and $\mathrm{HOPG}$ substrates many fundamental questions remain. The possible explanations provided in the discussion have to be investigated in depth with for example electron diffraction measurements in order to provide detailed insight in the crystallinity of the samples at different temperatures. However it was clearly shown that substrate interfacial energy strongly influences the thermal behavior of $\mathrm{PI}_{76}-b$-PFS 76 crystals deposited on substrates. This is especially of interest for the development of semiconductor devices utilizing these nanostructured PFS block copolymers, in which excessive heat is often generated leading to a significant increase in environmental temperature. 


\subsection{Conclusions}

The self assembly of $\mathrm{PI}_{76}-b$ - $\mathrm{PFS}_{76}$ in a PI selective solvent is a crystallization driven process resulting in the formation of platelet-like crystals with a semi-crystalline PFS core and solvent swollen PI corona. AFM analysis of crystals deposited on either $\mathrm{SiO}_{2}$ or HOPG revealed that after drying they were identical in size. The averaged height and width were measured to be, $\sim 13 \mathrm{~nm}$ and $\sim 130 \mathrm{~nm}$, respectively. XPS of $\mathrm{PI}_{76}-b$-PFS 76 crystals deposited on $\mathrm{SiO}_{2}$ before and after $\mathrm{O}_{2}$-RIE confirmed the presence of a PI corona around a PFS core. The apparent melting temperature of the semi-crystalline PFS core was determined with NanoTA to be $122{ }^{\circ} \mathrm{C} \pm 10{ }^{\circ} \mathrm{C}$ for a relatively thick layer of crystals on top of each other. For single crystals no softening temperature could be detected. This was ascribed to the thickness of the crystals $(\sim 13 \mathrm{~nm})$ to be below the physical limit of the technique. In situ examination of $\mathrm{PI}_{76}-b$ - $\mathrm{PFS}_{76}$ crystals deposited on $\mathrm{SiO}_{2}$ or $\mathrm{HOPG}$ with temperature controlled AFM revealed different morphology changes, which were ascribed to the hydrophilic and hydrophobic nature of the used substrates, respectively. The melting transitions observed with NanoTA and temperature controlled AFM were in good agreement with DSC measurements for bulk $\mathrm{PI}_{76}-b$-PFS 76 samples. The observed differences in thermal behavior of $\mathrm{PI}_{76}-b$ - $\mathrm{PFS}_{76}$ crystals deposited on $\mathrm{SiO}_{2}$ and HOPG emphasize the role of interfacial energy between polymer based nanostructures and supporting substrates with respect to the stability of the deposited nanostructures.

\subsection{Experimental}

Materials: The $\mathrm{PI}_{76}-b$ - $\mathrm{PFS}_{76}$ diblock copolymer with narrow size distribution was synthesized by sequential living anionic polymerization in THF in the group of Manners as described elsewhere. ${ }^{35,51,52}$ PI- $b$-PFS platelet-like crystals were prepared by dissolving $2.2 \mathrm{mg} \mathrm{PI} 76$ - $b$ - $\mathrm{PFS}_{76}$ in a mixture of decane $(20 \mathrm{ml})$ and xylene $(2 \mathrm{ml})$ in a vial thermostated at $100{ }^{\circ} \mathrm{C}$ (oil bath). Following complete dissolution of the block copolymer the temperature was lowered to $70{ }^{\circ} \mathrm{C}$ and kept for 12 hours. Subsequently the solution was cooled to room temperature. $\mathrm{PI}_{76}-b$ - $\mathrm{PFS}_{76}$ crystals were deposited on $1 \mathrm{~cm} \times 1 \mathrm{~cm}$ silicon (CZ, type $\mathrm{P}$, Boron, $<100>$, diameter $100 \mathrm{~mm}$, OKMETIC, Vantaa, Finland) or freshly cleaved HOPG (Agar Scientific Limited, Stansted, United Kingdom) substrates via drop casting or spin coating (1500 rpm, 30 seconds, Suss MicroTec Delta20 spincoater, Garching/Hochbrück, Germany) a $\sim 0.5 \mathrm{ml}$ aliquot of the crystal solution. The samples were allowed to dry in air followed by drying in vacuum at $\sim 60{ }^{\circ} \mathrm{C}$ for 12 hours. Silicon surfaces were extensively rinsed with toluene and ethanol before drying under a nitrogen stream prior to use. 
Temperature controlled AFM: The thermal behavior of the platelet $\mathrm{PI}_{76}-b$-PFS 76 crystals on silicon or HOPG supports was studied with a Multimode Nanoscope IIIa (Veeco/Digital Instruments (DI), Santa Barbara, CA, USA) AFM equipped with a Digital Instruments Multimode Heater/Cooler accessory (Veeco/Digital Instruments (DI), Santa Barbara, CA, USA). The latter primary consists of a sample heater and a probe holder with integrated cantilever heater. During the experiments the sample heater and probe holder were set to the same temperatures. The AFM was operated in tapping mode using silicon cantilevers with a resonance frequency and spring constant of $\sim 330 \mathrm{kHz}$ and $\sim 42 \mathrm{~N} \mathrm{~m}^{-1}$, respectively (PPP-NCH-W, Nanosensors, Wetzlar, Germany). Images under ambient conditions were taken at temperatures ranging from room temperature up to $160{ }^{\circ} \mathrm{C}$ after 10 minutes thermal equilibration.

Nanoscale thermal analysis: Heatable AFM probes (type AN-2, with $200 \mu \mathrm{m}$ long cantilevers, Anasys Instruments, Santa Barbara, CA, USA) mounted in a Dimension D3100 equipped with a hybrid 153 scanner and a NanoScope IVa controller (Veeco/Digital Instruments (DI), Santa Barbara, CA, USA) were used for the highly localized nanoscale thermal analysis of drop casted $\mathrm{PI}_{76}-b$ - $\mathrm{PFS}_{76}$ crystals on silicon. The probe temperature was controlled with a Nano-TA2 controller (Anasys Instruments, Santa Barbara, CA, USA). The temperature was ramped from $60{ }^{\circ} \mathrm{C}$ to $240{ }^{\circ} \mathrm{C}$ at $10{ }^{\circ} \mathrm{C} \mathrm{s}^{-1}$. Prior to the experiments the probe temperature was calibrated using polymer standards with known melting points: poly( $\varepsilon$-caprolactone) (PCL, $\left.T_{m}=55^{\circ} \mathrm{C}\right)$, poly (ethylene) $\left(\mathrm{PE}, T_{m}=116^{\circ} \mathrm{C}\right)$ and poly (ethylene terephthalate) (PET, $T_{m}=235^{\circ} \mathrm{C}$ ).

Differential scanning calorimetry (DSC): Bulk $\mathrm{PI}_{76}-b-\mathrm{PFS}_{76}$ samples of about $5 \mathrm{mg}$ were placed in a Perkin-Elmer DSC-7 (Waltham, MA, USA). After erasing the thermal history of the sample at $150{ }^{\circ} \mathrm{C}$ for $5 \mathrm{~min}$ the samples were isothermally crystallized at $\mathrm{T}_{\mathrm{c}}$ (between $80{ }^{\circ} \mathrm{C}$ up to $110{ }^{\circ} \mathrm{C}$ ) for $120 \mathrm{~min}$. Cooling the melt to $\mathrm{T}_{\mathrm{c}}$ was done at $200{ }^{\circ} \mathrm{C} \mathrm{min}^{-1}$. From $T_{c}$ the sample was heated at $10{ }^{\circ} \mathrm{C} \min ^{-1}$ to $150{ }^{\circ} \mathrm{C}$ in order to observe the melting transition.

$O_{2}$-RIE: $\mathrm{PI}_{76}-b$ - $\mathrm{PFS}_{76}$ crystals on silicon were exposed to an oxygen plasma in a reactive ion etch $\left(\mathrm{O}_{2}\right.$-RIE) setup (Elektrotech PF340, London, United Kingdom) for 30 seconds. The setup was operated at $50 \mathrm{~W}, 10 \mathrm{mTorr}$ and $20 \% \mathrm{O}_{2}$.

$X$-ray photoelectron spectroscopy: XPS spectra of untreated and heated $\mathrm{PI}_{76}-b$-PFS 76 crystals deposited on $\mathrm{SiO}_{2}$ and $\mathrm{HOPG}$ as well as of plasma etched $\mathrm{PI}_{76}-b$-PFS 76 crystals on $\mathrm{SiO}_{2}$ were recorded on a PHI Quantera XPS microprobe (Physical Electronics Inc., Chanhassen, MN, USA) using a monochromatic X-ray beam (Al Ka monochromatic at $1486.6 \mathrm{eV}, 100 \mu \mathrm{m}$ diameter, $25 \mathrm{~W}$ ) at a fixed take-off angle of $45^{\circ}$ $(\varphi)$ immediately after sample preparation. Charging of samples was corrected by shifting the peak positions relative to the position of neutral carbon at $285.0 \mathrm{eV}(\mathrm{C} 1 \mathrm{~s})$. 
Atomic concentrations were determined by numerical integration of the relative peak areas using the Mulitpak software with supplied atomic sensitivity factors relative to F1s (C1s: 0.314; O1s: 0.733; Si2p: 0.368; Fe2p: 2.946). ${ }^{53}$

Contact angle measurements: Static contact angles of PI and quenched melts of PFS on $\mathrm{SiO}_{2}$ and HOPG were measured with a CA microscope (Data Physics OCA model 15 Plus, Filderstadt, Germany) at room temperature. PFS samples were molten at $165{ }^{\circ} \mathrm{C}$ for 5 minutes and quenched to room temperature. PI was not additionally heated. Data for 3 different spots for each substrate were averaged. Silicon samples were cleaned by rinsing with toluene and ethanol followed by drying under a nitrogen stream. HOPG substrates were freshly cleaved prior to use.

\subsection{References}

1 Kim, Y.; Dalhaimer, P.; Christian, D. A.; Discher, D. E. Nanotechnology 2005, 16, S484.

2 Jo, Y. S.; van der Vlies, A. J.; Gantz, J.; Thacher, T. N.; Antonijevic, S.; Cavadini, S.; Demurtas, D.; Stergiopulos, N.; Hubbell, J. A. J. Am. Chem. Soc. 2009, 131, 14413.

3 Lee, Y.; Ishii, T.; Kim, H. J.; Nishiyama, N.; Hayakawa, Y.; Itaka, K.; Kataoka, K. Angew. Chem. Int. Ed. 2010, 49, 2552.

4 Quan, C. Y.; Chen, J. X.; Wang, H. Y.; Li, C.; Chang, C.; Zhang, X. Z.; Zhuo, R. X. ACS Nano 2010, 4, 4211.

5 Ge, J.; Lu, D. N.; Liu, Z. X.; Liu, Z. Biochem. Eng. J. 2009, 44, 53.

6 Mai, Y.; Eisenberg, A. J. Am. Chem. Soc. 2010, 132, 10078.

7 Chen, Q.; Schönherr, H.; Vancso, G. J. Small 2009, 5, 1436.

8 Cui, C. Z.; Bonder, E. M.; Jakle, F. J. Am. Chem. Soc. 2010, 132, 1810.

9 Glass, R.; Möller, M.; Spatz, J. P. Nanotechnology 2003, 14, 1153.

10 Bansmann, J.; Kielbassa, S.; Hoster, H.; Weigl, F.; Boyen, H. G.; Wiedwald, U.; Ziemann, P.; Behm, R. J. Langmuir 2007, 23, 10150.

11 Hu, Y. X.; Chen, D. A.; Park, S.; Emrick, T.; Russell, T. P. Adv. Mater. 2010, 22, 2583.

12 Geng, Y.; Dalhaimer, P.; Cai, S. S.; Tsai, R.; Tewari, M.; Minko, T.; Discher, D. E. Nat. Nanotechnol. 2007, 2, 249.

13 Aydin, D.; Schwieder, M.; Louban, I.; Knoppe, S.; Ulmer, J.; Haas, T. L.; Walczak, H.; Spatz, J. P. Small 2009, 5, 1014.

14 Park, M.; Harrison, C.; Chaikin, P. M.; Register, R. A.; Adamson, D. H. Science 1997, 276, 1401.

15 Korczagin, I.; Lammertink, R. G. H.; Hempenius, M. A.; Golze, S.; Vancso, G. J. Adv. Polym. Sci. 2006, 200, 91.

16 Darling, S. B. Prog. Polym. Sci. 2007, 32, 1152.

17 Park, C.; Yoon, J.; Thomas, E. L. Polymer 2003, 44, 6725.

18 Hamley, I. W. Prog. Polym. Sci. 2009, 34, 1161.

19 Ross, C. A.; Jung, Y. S.; Chuang, V. P.; Ilievski, F.; Yang, J. K. W.; Bita, I.; Thomas, E. L.; Smith, H. I.; Berggren, K. K.; Vancso, G. J.; Cheng, J. Y. J. Vac. Sci. Technol. B 2008, 26, 2489. 
Korczagin, I.; Hempenius, M. A.; Fokkink, R. G.; Stuart, M. A. C.; Al-Hussein, M.; Bomans, P. H. H.; Frederik, P. M.; Vancso, G. J. Macromolecules 2006, 39, 2306. Kulbaba, K.; Manners, I. Macromol. Rapid Commun. 2001, 22, 711. Shen, L.; Wang, H.; Guerin, G.; Wu, C.; Manners, I.; Winnik, M. A. Macromolecules 2008, 41, 4380 .

Qian, J. S.; Zhang, M.; Manners, I.; Winnik, M. A. Trends Biotechnol. 2010, 28, 84. Hempenius, M. A.; Cirmi, C.; Song, J.; Vancso, G. J. Macromolecules 2009, 42, 2324.

Shi, W. Q.; Giannotti, M. I.; Zhang, X.; Hempenius, M. A.; Schönherr, H.; Vancso, G. J. Angew. Chem. Int. Ed. 2007, 46, 8400.

Wang, H.; Wang, X. S.; Winnik, M. A.; Manners, I. J. Am. Chem. Soc. 2008, 130, 12921. Acikgoz, C.; Ling, X. Y.; Phang, I. Y.; Hempenius, M. A.; Reinhoudt, D. N.; Huskens, J.; Vancso, G. J. Adv. Mater. 2009, 21, 2064.

28 Lammertink, R. G. H.; Hempenius, M. A.; Chan, V. Z. H.; Thomas, E. L.; Vancso, G. J. Chem. Mater. 2001, 13, 429.

Cao, L.; Massey, J. A.; Winnik, M. A.; Manners, I.; Riethmüller, S.; Banhart, F.; Spatz, J. P.; Möller, M. Adv. Funct. Mater. 2003, 13, 271.

30 Massey, J. A.; Winnik, M. A.; Manners, I.; Chan, V. Z. H.; Ostermann, J. M.; Enchelmaier, R.; Spatz, J. P.; Möller, M. J. Am. Chem. Soc. 2001, 123, 3147.

31 Wang, X. S.; Arsenault, A.; Ozin, G. A.; Winnik, M. A.; Manners, I. J. Am. Chem. Soc. 2003, $125,12686$.

Korczagin, I.; Hempenius, M. A.; Vancso, G. J. Macromolecules 2004, 37, 1686. Rider, D. A.; Manners, I. Polym. Rev. 2007, 47, 165.

34 Manners, I. Chem. Comm. 1999, 857.

35 Massey, J. A.; Temple, K.; Cao, L.; Rharbi, Y.; Raez, J.; Winnik, M. A.; Manners, I. J. Am. Chem. Soc. 2000, 122, 11577.

36 Raez, J.; Manners, I.; Winnik, M. A. J. Am. Chem. Soc. 2002, 124, 10381.

37 Rasburn, J.; Petersen, R.; Jahr, T.; Rulkens, R.; Manners, I.; Vancso, G. J. Chem. Mater. 1995, 7, 871.

38 Rulkens, R.; Lough, A. J.; Manners, I.; Lovelace, S. R.; Grant, C.; Geiger, W. E. J. Am. Chem. Soc. 1996, 118, 12683.

39 Wang, X. S.; Liu, K.; Arsenault, A. C.; Rider, D. A.; Ozin, G. A.; Winnik, M. A.; Manners, I. J. Am. Chem. Soc. 2007, 129, 5630.

40 Lammertink, R. G. H.; Hempenius, M. A.; Vancso, G. J. Macromol. Chem. Phys. 1998, 199, 2141.

41 Lammertink, R. G. H.; Hempenius, M. A.; Manners, I.; Vancso, G. J. Macromolecules 1998, 31, 795.

42 Xu, J. J.; Bellas, V.; Jungnickel, B.; Stuhn, B.; Rehahn, M. Macromol. Chem. Phys. 2010, 211, 1261.

43 Zhang, F. J.; Stühn, B. Colloid Polym. Sc.i 2007, 285, 371.

44 Hoffman, J. D.; Weeks, J. J. J. Res. Nbs. a Phys. Ch. 1962, 66A, 13.

45 Zhou, H. Y.; Wilkes, G. L. Polymer 1997, 38, 5735.

46 Nelson, B. A.; King, W. P. Rev. Sci. Instrum. 2007, 78, 023702.

47 Zhou, J.; Berry, B.; Douglas, J. F.; Karim, A.; Snyder, C. R.; Soles, C. Nanotechnology 2008, 19,495703 . 
48 Briggs, D.; Seah, M. P. Practical Surface Analysis: by Auger and X-ray Photoelectron Spectroscopy, Wiley, Chichester, UK, 1983.

49 Stark, R.; Bonaccurso, E.; Kappl, M.; Butt, H. J. Polymer 2006, 47, 7259.

50 Roerdink, M.; Hempenius, M. A.; Gunst, U.; Arlinghaus, H. F.; Vancso, G. J. Small 2007, 3, 1415.

51 Ni, Y. Z.; Rulkens, R.; Manners, I. J. Am. Chem. Soc. 1996, 118, 4102.

52 Gädt, T.; Ieong, N. S.; Cambridge, G.; Winnik, M. A.; Manners, I. Nat. Mater. 2009, 8, 144.

53 Zhao, J.; Garza, E. G.; Lam, K.; Jones, C. M. Appl. Surf. Sci. 2000, 158, 246. 


\section{Chapter 6}

\section{Reactive Imprint Lithography: Combined Topographical Patterning and Chemical Surface Functionalization of Poly(styrene)-block-poly(tert-butyl acrylate) Films}

In this chapter we introduce Reactive Imprint Lithography (RIL) as a new onestep lithographic tool for the fabrication of large area topographically patterned, chemically activated polymer platforms. Films of poly(styrene)-block-poly(tert-butyl acrylate) (PS- $b$-P $t$ BA) were imprinted with poly(dimethylsiloxane) (PDMS) master stamps at temperatures above the corresponding glass transition and chemical deprotection temperatures to yield structured films with exposed carboxylic acid and anhydride groups. Faithful pattern transfer was confirmed by atomic force microscopy (AFM) analyses. Transmission mode FTIR spectra showed a conversion of over $95 \%$ of the tert-butyl ester groups after RIL at $230{ }^{\circ} \mathrm{C}$ for 5 minutes and a significantly reduced conversion to anhydride compared to thermolysis of neat films with free surfaces in air or nitrogen. An enrichment of the surface layer in PS was detected by angle resolved X-ray photoelectron spectroscopy (XPS). In order to demonstrate application potentials of the activated platforms a $7 \mathrm{~nm} \pm 1 \mathrm{~nm}$ thick $\mathrm{NH}_{2}$-terminated PEG layer (grafting density of 0.9 chains $\mathrm{nm}^{-2}$ ) was covalently grafted to RIL activated substrates. This layer reduced the non-specific adsorption (NSA) of bovine serum albumin (BSA) by $95 \%$ to a residual mass coverage of $9.1 \pm 2.9 \mathrm{ng} \mathrm{cm}^{-2}$. As shown by these examples RIL comprises an attractive complementary approach to produce bio-reactive polymer surfaces with topographic patterns in a one-step process. In addition, the controlled thermochemical surface functionalization of the block copolymer (BCP) film platform yields promises for the development of scanning thermal lithography on BCP films.

\footnotetext{
* Parts of this chapter have been published in: Duvigneau, J.; Cornelissen, S.; Bardjí Valls, N.; Schönherr, H.; Vancso, G. J. Adv. Funct. Mater. 2010, 20, 460.
} 


\subsection{Introduction}

Topographical and/or chemical patterning of soft condensed matter from the micrometer down to the nanometer length scales is important for the development of biologically relevant interfaces $e$.g. for fundamental cell-surface interaction studies, ${ }^{1}$ also in the context of tissue engineering, ${ }^{2}$ advanced biosensors, ${ }^{3}$ etc. In particular, for cell-surface interaction studies it is imperative to control the topography and chemical surface functionality over the mentioned length scales simultaneously. ${ }^{4}$

Among the currently available approaches to obtain well defined patterned substrates one can differentiate (i) massively parallel and (ii) serial approaches. Former ones include conventional photolithography ${ }^{5}$ and more recently introduced technologies, such as microcontact printing $(\mu \mathrm{CP})^{6}$ and nano-imprint lithography (NIL), ${ }^{7}$ while the serial approaches comprise e.g. directed beam ${ }^{8}$ and scanning probe lithography. ${ }^{9}$ Self-assembly is considered to be a third complementary way to fabricate the required patterns. ${ }^{10}$

The need for simultaneous topographic and chemical patterns has been addressed previously by sequential processing steps. Examples include the directed self assembly of binary poly(styrene) - (polyvinyl pyridine) (PS-PVP) blends on thiol templated gold followed by selective removal of the $\mathrm{PVP}^{11}$ and photolithographically structured SU8 photoresist/glass films in which the exposed glass was subsequently functionalized with physisorbed poly(L-lysine). ${ }^{12}$ Mrksich et al. ${ }^{13}$ utilized several sequential $\mu \mathrm{CP}$ and micro molding steps to control micrometer scale topology and interfacial characteristics of gold coated polyurethane substrates. Block copolymers were also patterned by illuminating dispersed photoacid generators through a mask. ${ }^{14}$ The Textor group has introduced inverted $\mu \mathrm{CP},{ }^{15}$ which combines topography imprinting followed by the selective contact printing of (bio)molecules on top of the mesas obtained by imprinting.

In many established methods that are used to topographically structure materials, such as the mentioned polymer (nano)imprinting, ${ }^{7 \mathrm{~d}, 16,17}$ heat is exploited. Thermally induced chemical reactions and in particular surface chemical reactions on the other hand have received considerably less attention (see also Chapter 1). Böker et al. reported on the thermal cleavage of the perfluorinated side chains of poly(styrene)block-poly(isoprene[graft-perfluoroacyl]) block copolymer surfaces at $340{ }^{\circ} \mathrm{C}$ and mentioned possible application for covalent surface grafting of molecules. ${ }^{18}$ Nealey and coworkers induced a transition from a cylindrical to a spherical morphology in poly(styrene)-block-poly(tert-butyl acrylate) block copolymer thin films by thermal cleavage of the tert-butyl ester in the tert-butyl acrylate block at $160{ }^{\circ} \mathrm{C} .{ }^{19}$ Finally, the 
local thermal surface activation through deprotection of ester-protected carboxylic acid groups by thermal scanning probe lithography at temperatures above $150{ }^{\circ} \mathrm{C}$ has been reported by King and coworkers ${ }^{20}$ and by our group. ${ }^{21}$

Here we introduce a new approach for the combined topographical patterning and chemical functionalization of large area polymer films in a single-step imprinting process at elevated temperatures. The principle of the approach termed Reactive Imprint Lithography (RIL) exploits the simultaneously occurring deprotection reaction of tert-butyl ester groups during (nano)imprinting (Figure 6.1). As discussed in this chapter, the structured and activated surfaces thus obtained can be functionalized conveniently with established surface chemistry to yield functional biointerfaces.

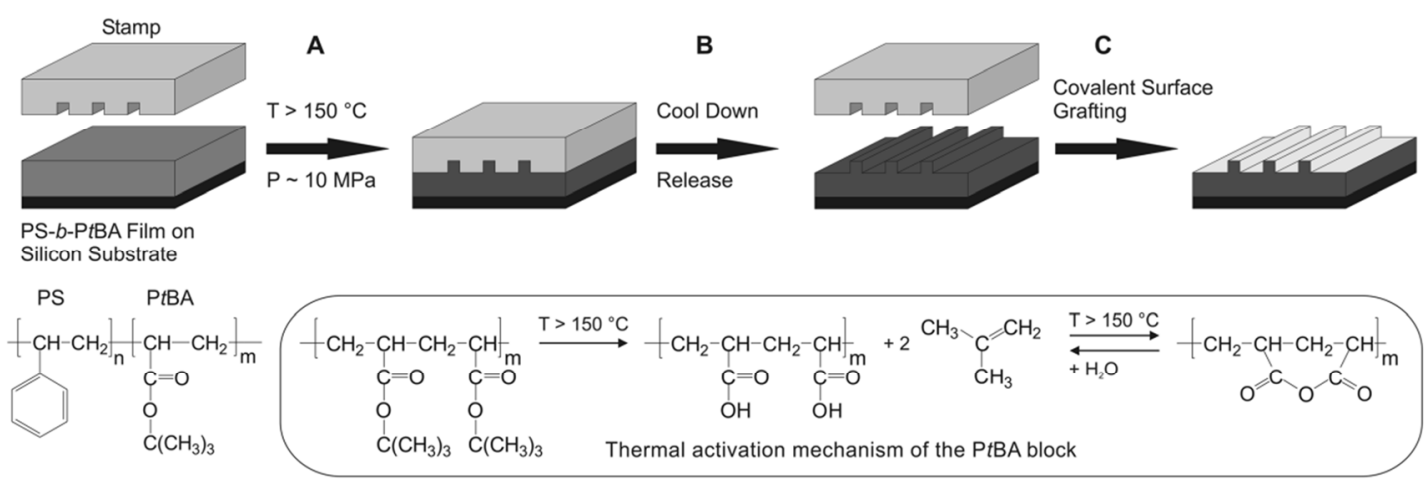

Figure 6.1: Schematic of reactive imprint lithography. (A) PDMS stamps are used to imprint PS-b-PtBA polymer films at temperatures above $150{ }^{\circ} \mathrm{C}$. (B) After cooling and demolding a carboxylic acid functionalized topographically patterned film is obtained. (C) Wet chemical derivatization with e.g. $\mathrm{NH}_{2}$-terminated PEG or proteins affords topographically structured and (bio)chemically functional surfaces.

\subsection{Results and discussion}

RIL comprises the simultaneous topographical patterning by imprint lithography and chemical activation of PS- $b$-P $t$ BA films at temperatures above $150{ }^{\circ} \mathrm{C}$. A prerequisite for the master stamp material used, besides good mechanical stability and conformal stamp - film contact, is the permeability for the gaseous reaction product (isobutylene). ${ }^{21}$ Imprinting with impermeable silicon stamps resulted in a distorted pattern with as yet unexplained morphology (data not shown). Masters fabricated from PDMS elastomer fulfill the requirement of permeability. ${ }^{22}$ Furthermore topographic feature sizes can easily be controlled over a broad range of length scales down to several $100 \mathrm{~nm}$ or even below. ${ }^{23}$ 
RIL and the underlying processes were investigated by analyzing first the topography of the masters and films prepared under different conditions. Subsequently, the deprotection reaction was elucidated by FTIR and angle-dependent XPS spectroscopic analyses, followed by surface chemical modification and an assessment of the films' functionality.

\subsubsection{AFM investigation of the pattern transfer to $\mathrm{PS}_{2092}-b-\mathrm{P} t \mathrm{~B} \mathrm{~A}_{1055}$ films during RIL}

Topographically patterned PDMS stamps were used to imprint $\mathrm{PS}_{2092}-b-\mathrm{P} \mathrm{BA}_{1055}$ polymer films above the glass transition temperature of both blocks $\left(100{ }^{\circ} \mathrm{C}\right.$ and $43{ }^{\circ} \mathrm{C}$ for PS and $\mathrm{P} t \mathrm{BA}$, respectively $)^{24}$ and above the thermal deprotection temperature of the tert-butyl ester of $150{ }^{\circ} \mathrm{C} .{ }^{21}$ At $230{ }^{\circ} \mathrm{C}$ thin films were shown to be practically completely thermolyzed after 5 minutes (see section 6.2.2). Two different temperature profiles were investigated to establish whether there is any difference in the order of the two processes:

RIL\#1: imprinting at $150{ }^{\circ} \mathrm{C}$ for $40 \mathrm{~min}$ followed by $230{ }^{\circ} \mathrm{C}$ for $5 \mathrm{~min}$.

RIL\#2: imprinting at $230^{\circ} \mathrm{C}$ for $5 \mathrm{~min}$ followed by $150^{\circ} \mathrm{C}$ for $40 \mathrm{~min}$.

Imprinting PS- $b$-P $t$ BA films at $150{ }^{\circ} \mathrm{C}$ was expected to provide mainly pattern transfer, ${ }^{25}$ while imprinting at $230{ }^{\circ} \mathrm{C}$ may afford pattern transfer combined with thermal deprotection of the tert-butyl ester. ${ }^{21}$ Prolonged thermolysis times at $230{ }^{\circ} \mathrm{C}$ are known to result in unwanted decarboxylation, ${ }^{21}$ which would lower the concentration of carboxylic acid groups in the film.

Table 6.1: AFM analysis of the ridge height, ridge width and periodicity for the silicon master, $P S_{2092-b-P t B A} 1055$ polymer films after RIL and PDMS stamps before and after RIL. The stated error values are standard deviations $(n=9)$.

\begin{tabular}{l|c|c|c}
\hline & $\begin{array}{c}\text { Ridge height } \\
{[\mu \mathrm{m}]}\end{array}$ & $\begin{array}{c}\text { Ridge width } \\
{[\mu \mathrm{m}]}\end{array}$ & $\begin{array}{c}\text { Periodicity } \\
{[\mu \mathrm{m}]}\end{array}$ \\
\hline \hline Silicon Master & $1.04 \pm 0.03$ & $6.1 \pm 0.1$ & $19.2 \pm 0.1$ \\
RIL\#1 & $0.98 \pm 0.01$ & $6.1 \pm 0.2$ & $19.5 \pm 0.2$ \\
RIL\#2 & $1.04 \pm 0.03$ & $6.0 \pm 0.1$ & $19.6 \pm 0.3$ \\
PDMS stamp before RIL & $0.99 \pm 0.01$ & $13.8 \pm 0.1$ & $19.1 \pm 0.2$ \\
PDMS stamp after RIL\#1 & $1.02 \pm 0.01$ & $13.6 \pm 0.2$ & $19.0 \pm 0.3$ \\
PDMS stamp after RIL\#2 & $1.06 \pm 0.01$ & $14.0 \pm 0.1$ & $19.0 \pm 0.2$
\end{tabular}


Figure 6.2 shows tapping mode AFM height images and corresponding crosssections of the line patterned silicon master, a PDMS stamp before use and two $\mathrm{PS}_{2092}-b-\mathrm{P} t \mathrm{BA}_{1055}$ films after RIL\#1 and RIL\#2, respectively. The measured ridge heights, ridge widths and periodicities, as were analyzed from the corresponding AFM height images, are summarized in Table 6.1.
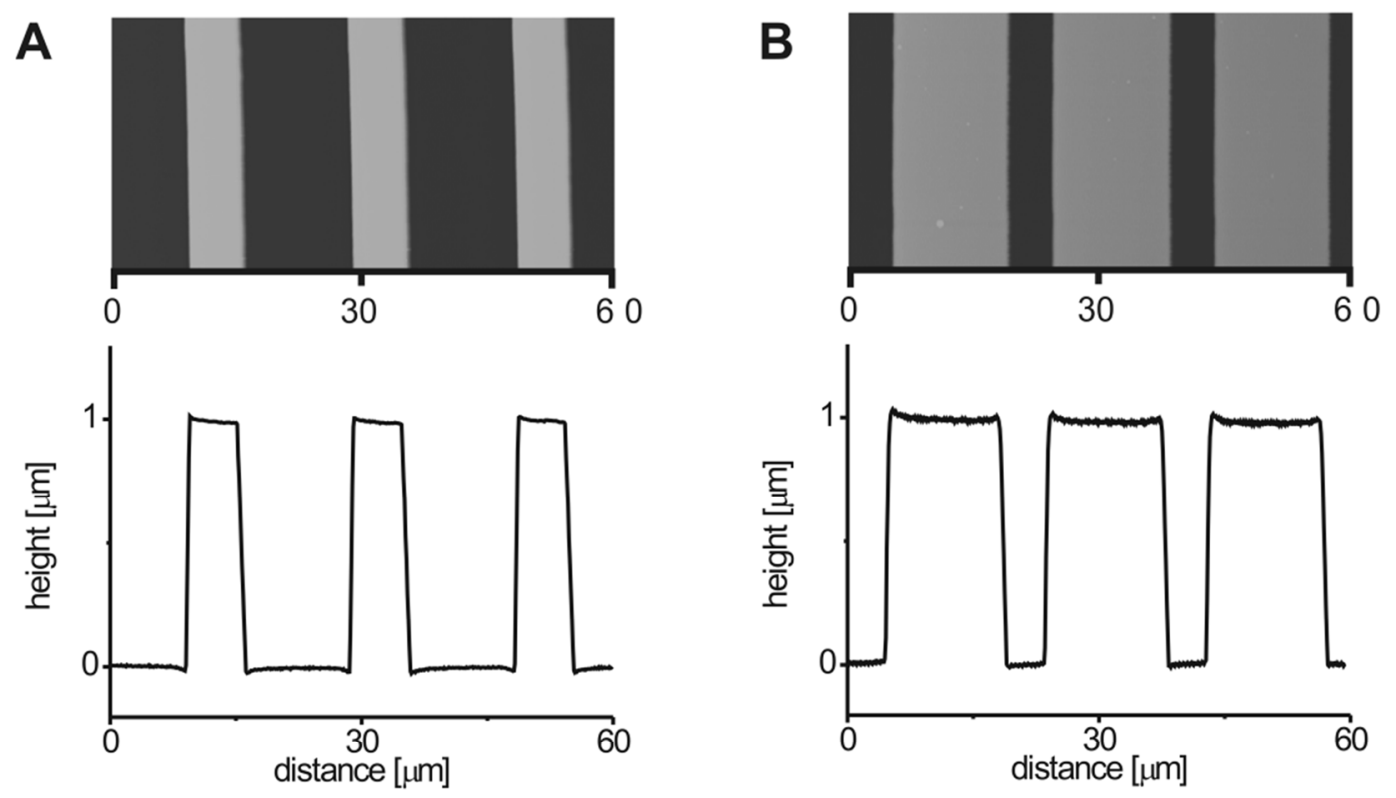

C
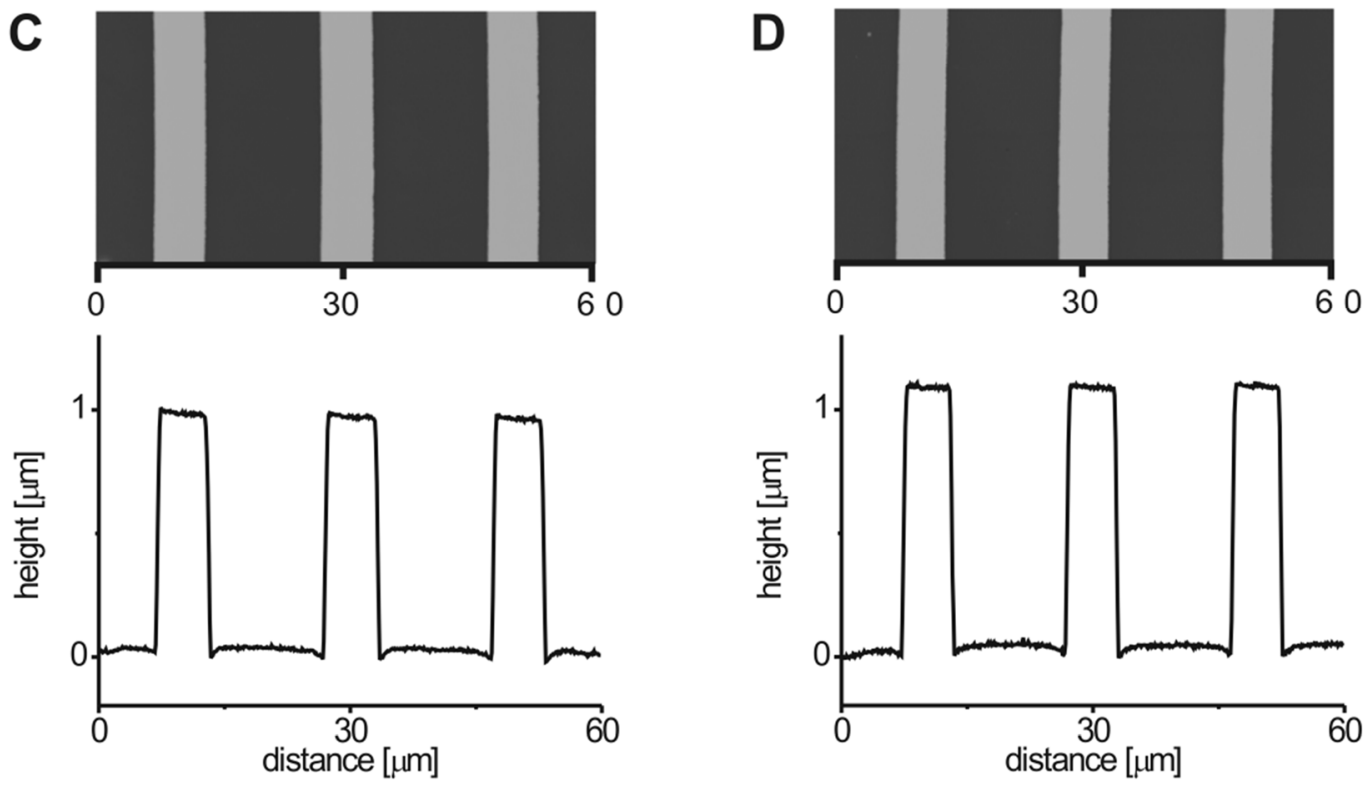

Figure 6.2: Tapping mode AFM height images (top) and horizontal cross-sections (bottom) of (A) the silicon master, (B) PDMS stamp replicated from the silicon master and $P S_{2092}-b-P t B A_{1055}$ polymer films after (C) RIL\#1 and (D) RIL\#2, respectively. 
Line patterns (ridge width $6.1 \mu \mathrm{m}$, ridge height $1.0 \mu \mathrm{m}$, periodicity $19.2 \mu \mathrm{m}$ ) were successfully replicated in the films via RIL. Compared to the silicon master, the observed deviations in ridge width and height were to within the experimental error of the analysis, regardless of the sequence of the thermal activation and imprinting for both temperature profiles. The slight increase in periodicity is in accordance with the expected thermal expansion of the PDMS stamp during RIL. ${ }^{26}$

\subsubsection{Transmission FTIR spectroscopy on $\mathbf{P S}_{2092}-b-P t B A_{1055}$ films after RIL}

The conversion of the PtBA block to poly(acrylic acid) (PAA) throughout the films during RIL was quantitatively analyzed by transmission FTIR spectroscopy. A detailed investigation of the proposed thermolysis reaction mechanism is provided in Chapter 7. Figure 6.3 shows FTIR spectra for films prior to and after RIL for 5 minutes at $230{ }^{\circ} \mathrm{C}$. The decrease of absorbances corresponding to $\delta_{\mathrm{C}-\mathrm{H}}$ vibrations of the tert-butyl ester methyl groups $\left(1350 \mathrm{~cm}^{-1}\right.$ and $\left.1368 \mathrm{~cm}^{-1}\right)$ after RIL indicates the conversion of tert-butyl ester groups to carboxylic acid groups. Carboxylic acid group formation is also evidenced from the shift of the $v_{\mathrm{C}=\mathrm{O}}$ (tert-butyl ester, $1733 \mathrm{~cm}^{-1}$, dashed line) to $1709 \mathrm{~cm}^{-1}$ ( $v_{\mathrm{C}=\mathrm{O}}$ carboxylic acid). The absorbances at $1752 \mathrm{~cm}^{-1}$ and $1804 \mathrm{~cm}^{-1}$ are attributed to the $v_{\mathrm{C}=\mathrm{O}}$ of anhydride groups. The conversion of the tert-butyl ester to carboxylic acid groups was estimated to be $>95 \%$ according to:

$$
X_{\text {tert-butylester }}=\left(1-\frac{\left(A_{1368} / A_{1455}\right)_{\text {RIL }}}{\left(A_{1368} / A_{1455}\right)_{\text {Annealed }}}\right) \times 100
$$

The normalization of the peak areas to the peak area of $v_{\mathrm{s}}(\mathrm{C}-\mathrm{C})$ at $1455 \mathrm{~cm}^{-1}$ of the poly(styrene) backbone corrects for possible slight film thickness differences.

Interestingly, the formation of anhydride is significantly suppressed compared to data provided in Chapter 7 (compare Figure 7.3) for films thermolyzed in a nitrogen atmosphere at $230{ }^{\circ} \mathrm{C}^{21}$ The peak areas of $v_{C=O}$ (anhydride) at $1804 \mathrm{~cm}^{-1}$ normalized with respect to the peak area of $v_{\mathrm{s}}(\mathrm{C}-\mathrm{C})$ at $1455 \mathrm{~cm}^{-1}$ of the poly(styrene) backbone within the same sample for films after thermolysis at $230{ }^{\circ} \mathrm{C}$ for 5 minutes in nitrogen, air or confinement with a flat PDMS stamp, were 2.1, 2.0 and 0.2, respectively. 


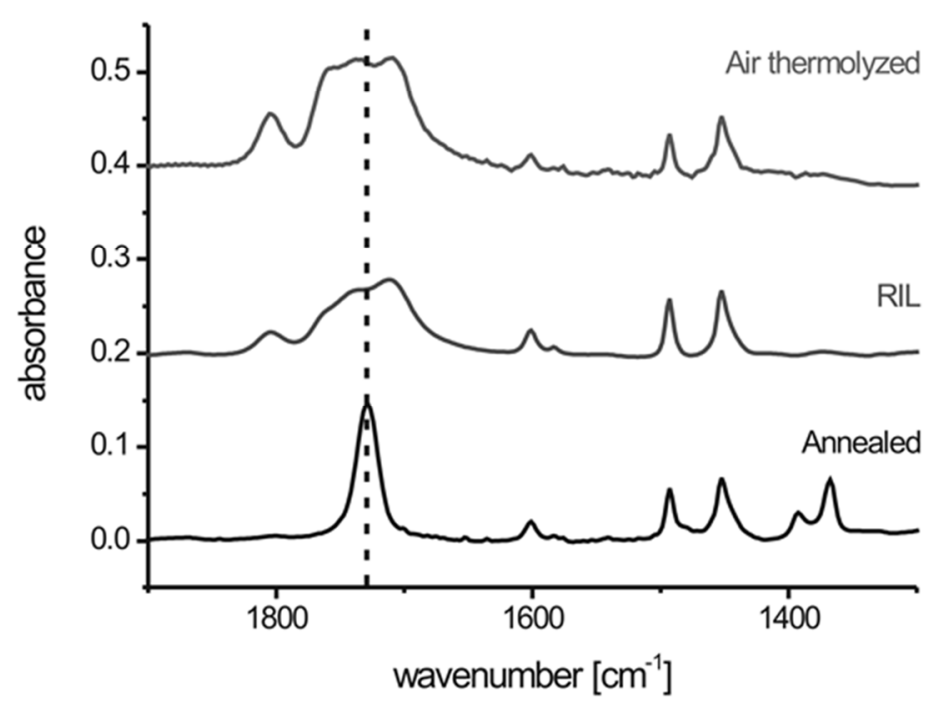

Figure 6.3: Transmission FTIR spectra of $P S_{2092}-b-P t B A_{1055}$ polymer films that were annealed (bottom), after RIL for $5 \mathrm{~min}$ at $230^{\circ} \mathrm{C}$ with a flat PDMS stamp (middle) and thermolyzed in air for 5 minutes at $230^{\circ} \mathrm{C}$ (top). Upon thermal deprotection of the PtBA block the $v_{C=0}$ (ester, $1733 \mathrm{~cm}^{-1}$, dashed line) shifts to $1709 \mathrm{~cm}^{-1}$ which corresponds to $v_{C=0}$ (carboxylic acid). The shoulder at $1752 \mathrm{~cm}^{-1}$ and $1804 \mathrm{~cm}^{-1}$, corresponds to absorbances of $v_{C=O}$ (anhydride).

The total fraction of anhydride formed during RIL for 5 minutes at $230{ }^{\circ} \mathrm{C}$ is thus a factor 10 less compared to thermolysis of similar films in an air or nitrogen atmosphere. This observation can be explained considering that the water formed upon anhydride formation during RIL is expected to reside preferentially in the hydrophilic PAA rather than diffuse through the hydrophobic PDMS stamp. ${ }^{27}$ The equilibrium between carboxylic acid groups and anhydride groups may thus be shifted towards the carboxylic acid groups. By contrast, when the thermolysis of the films is performed in the absence of PDMS stamps, the expected conversion to anhydride was observed. $^{21}$

\subsubsection{Surface layer composition and functionality investigated with angle resolved XPS}

The changes in chemical composition of the polymer film surfaces upon thermal activation and chemical derivatization were further investigated with angle resolved XPS (ARXPS). Figure 6.4 shows the detailed XPS C1s core spectra at a take-off angle $(\varphi)$ of $45^{\circ}$ of annealed PS- $b$-P $t$ BA before and after RIL, as well as after EDC/NHS activation followed by the wet chemical grafting of $\mathrm{PEG}_{113}-\mathrm{NH}_{2}$. 


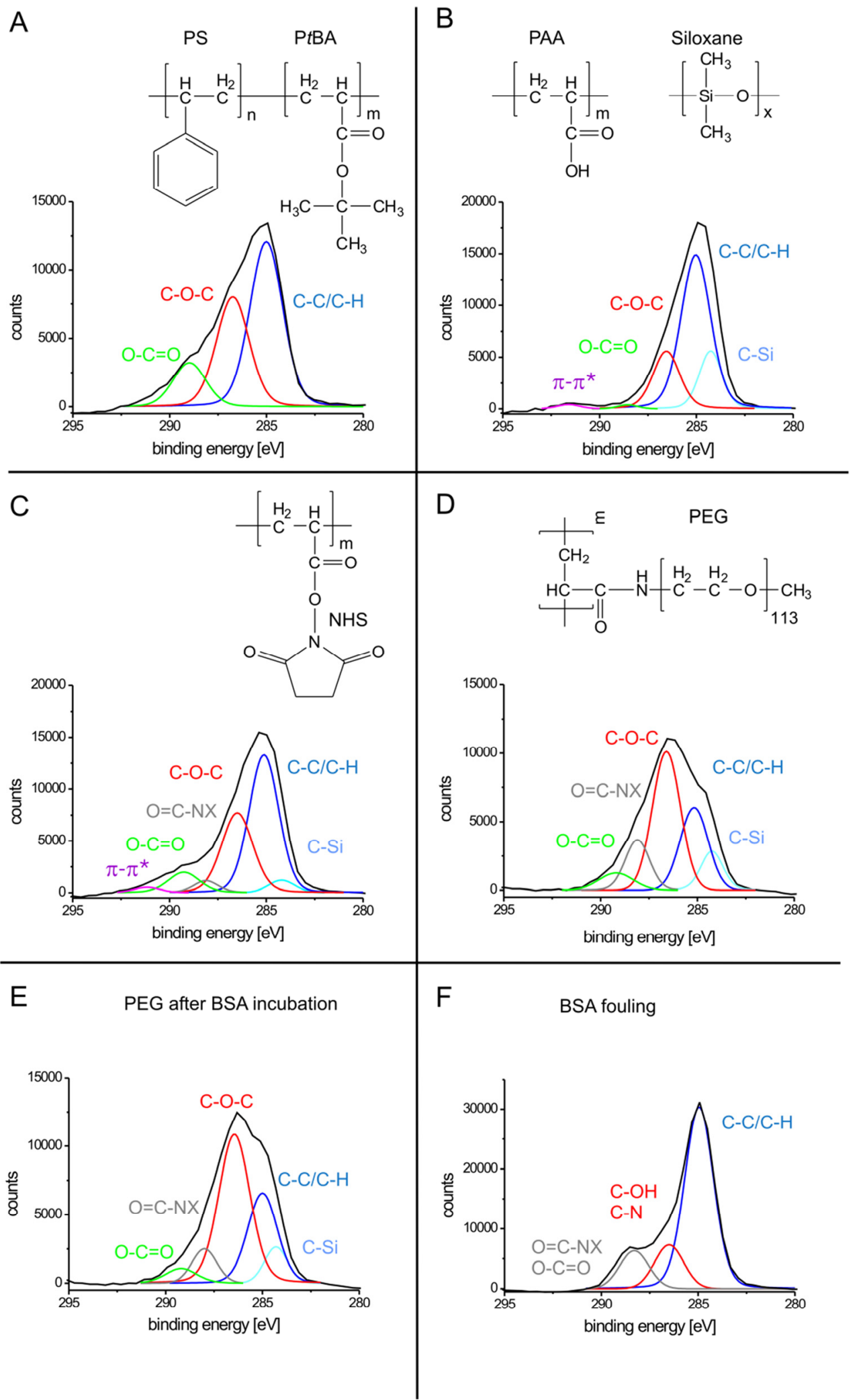

Figure 6.4: XPS C1s core spectra of annealed $P S_{2092}-b-P t B A_{1055}$ polymer films before $(A)$ and after RIL (B) acquired at $\varphi=45^{\circ}$. Following RIL the samples were activated with $E D C / N H S(C)$ and MeO-PEG $G_{113}-N_{2}$ was covalently immobilized (D). The PEG functionalized samples were immersed in a BSA solution (E) and compared to an annealed $P S_{2092}-b-$ $P t B A_{1055}$ film immersed in the same BSA solution (F). 
Figure 6.5 shows the angle resolved atomic $\mathrm{Si}$ concentrations and $\mathrm{C} / \mathrm{O}$ ratios for the samples mentioned above for $\varphi$ between $5^{\circ}$ and $70^{\circ}$. These take-off angles result in escape depths of the photoelectrons ranging from $\sim 1 \mathrm{~nm}$ to $\sim 9 \mathrm{~nm}^{28}$
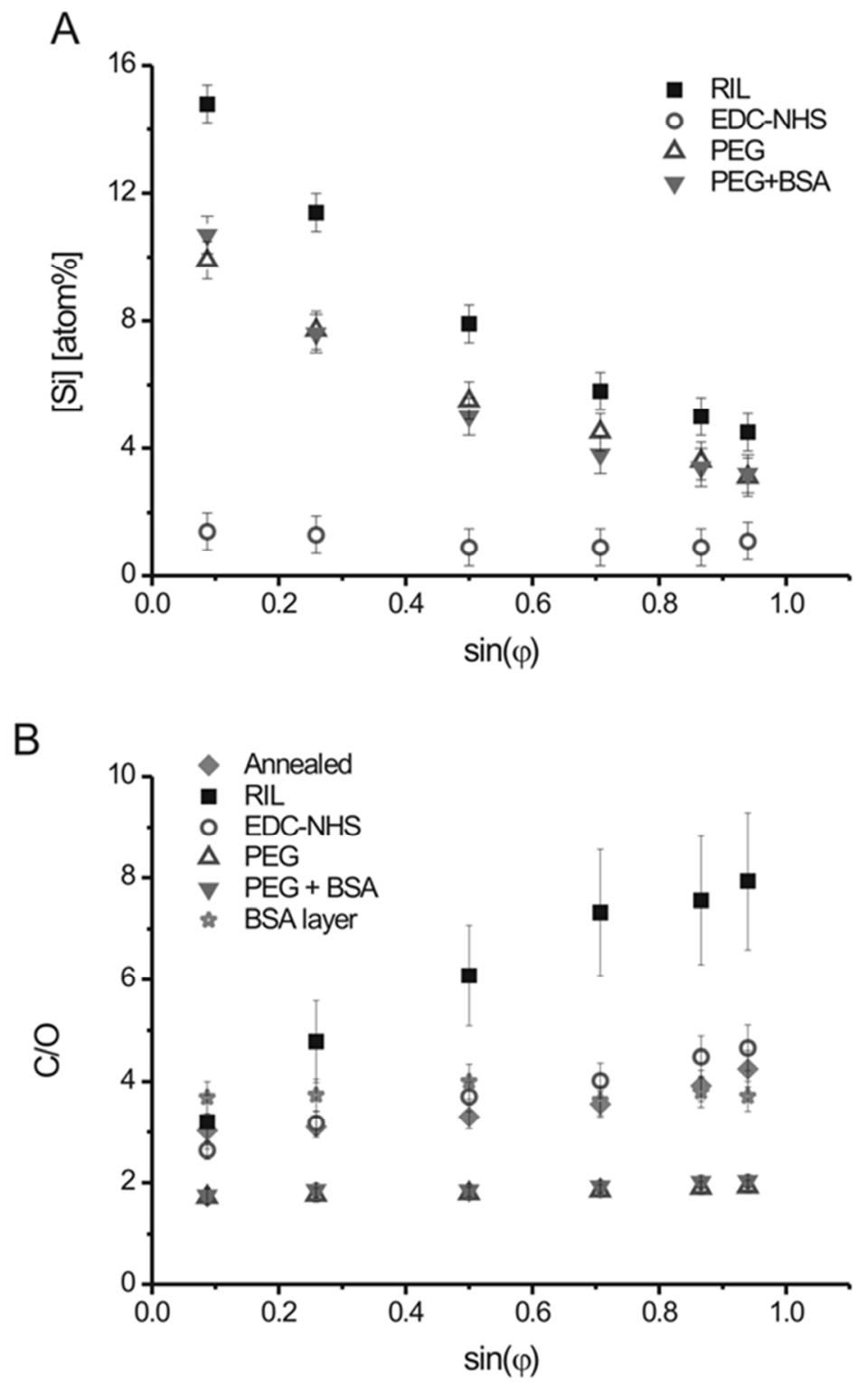

Figure 6.5: Silicon concentration $(A)$ and $C / O$ ratio $(B)$ as a function of the $\sin (\varphi)$ in ARXPS for $P S_{2092}-b-P t B A_{1055}$ polymer films after annealing, RIL, EDC/NHS activation, PEG grafting, immersion in a BSA solution and a full BSA layer, respectively.

Due to the lower surface energy of $\mathrm{P} t \mathrm{BA}\left(\gamma=31.2 \mathrm{mN} \mathrm{m}^{-1}\right)$ compared to PS $\left(\gamma=40.7 \mathrm{mN} \mathrm{m}^{-1}\right)$ the surface layer of the annealed films consists of $\mathrm{P} t \mathrm{BA}{ }^{24}$ This is evidenced by the presence of C-H/C-C, C-O-C and O-C $=\mathrm{O} \mathrm{C} 1 \mathrm{~s}$ peaks at $285.0 \mathrm{eV}$, $286.5 \mathrm{eV}$ and $289.0 \mathrm{eV}$, respectively and the lack of the typically observed $\pi$ - $\pi^{*}$ shake up peak for PS at $291.6 \mathrm{eV}$ (Figure 6.4A). The $\mathrm{C} / \mathrm{O}$ ratio for $\sin (\varphi)=0.7$ is 3.5 , which is in good agreement with the theoretically expected $\mathrm{C} / \mathrm{O}$ ratio for $\mathrm{P} t \mathrm{BA}$ 
homopolymer (3.5). For higher $\sin (\varphi)$ the $\mathrm{C} / \mathrm{O}$ ratio increases gradually, which is ascribed to the presence of the PS block. The PtBA skin layer thickness was estimated to be $8 \mathrm{~nm} \pm 1$ thick which corresponds very well with the value reported by Vancso et $a l$. for $\mathrm{PS}_{690}-b$ - $\mathrm{P} \mathrm{BA}_{1210}$ polymer films. ${ }^{29}$

Upon thermal activation at $230{ }^{\circ} \mathrm{C}$ for 5 minutes the surface layer of $\mathrm{PS}_{2092}-b-\mathrm{P} t \mathrm{BA}_{1055}$ films became enriched in PS, which is observed by the presence of the $\pi-\pi^{*}$ shake up peak at $291.6 \mathrm{eV}$, a decrease of the $\mathrm{O}-\mathrm{C}=\mathrm{O} \mathrm{C} 1 \mathrm{~s}$ peak at $289 \mathrm{eV}$ (Figure 6.4B), as well as an increase of the $\mathrm{C} / \mathrm{O}$ ratio for all investigated escape depths. By contrast, samples that were thermally activated in air revealed a significantly higher $\mathrm{C} / \mathrm{O}$ ratio and an increased $\pi-\pi^{*}$ shake up peak intensity at $\varphi=45^{\circ}$ (data not shown). Apparently, the thermal activation of P $t \mathrm{BA}$ in close contact with PDMS reduces the observed enrichment in PS, which is favorable for further derivatization.

RIL at $150{ }^{\circ} \mathrm{C}$ for prolonged times did not change the chemical composition of the surface layers besides a small fraction of silicon contamination (1.6 atom\%) when compared with an annealed film. This is in agreement with Tof-SIMS and water contact angle measurements previously reported by our group for imprinting PS- $b$-P $t$ BA films with PDMS stamps. ${ }^{25}$ Therefore it can be concluded that once the P $t$ BA block is deprotected, the surface layer becomes enriched in PS, presumably due to a lowering of the interfacial free energy. As a consequence the imprinting time after thermal activation should be as short as possible to obtain the highest fraction of carboxylic acid groups present at the surface after RIL. The presence of the C-O-C $\mathrm{C} 1 \mathrm{~s}$ peak at $286.5 \mathrm{eV}$ is in agreement with the incomplete conversion of the tert-butyl ester to carboxylic acid groups as was also observed with transmission FTIR spectroscopy. Typically around 6 atom $\%$ silicon contamination is present at $\varphi=45^{\circ}$ after RIL, which is related to highly mobile cyclic siloxanes and oligomeric PDMS fractions present in the PDMS stamps. ${ }^{30}$

Based on the known chemical composition and stoichiometry of these compounds, the PDMS contamination is around 24 atom $\%$ at this escape depth. From Figure 6.5 it is clear that the silicon concentration is highest after RIL, while after wet chemical grafting reactions the signal attributed to contamination is decreased. Also with increasing escape depth the overall observed silicon contamination decreases, which indicates that the majority of the PDMS contamination is present at top of the polymer films' surface. 
Following RIL the in situ formed carboxylic acid groups in the PS- $b$-P $t$ BA films were activated with NHS and EDC according to well known procedures. ${ }^{31}$ Subsequently, $\mathrm{PEG}_{113}-\mathrm{NH}_{2}$ (or fluoresceinamine, see below) was covalently immobilized on the surface of the film. EDC/NHS mediated activation of the carboxylic acid groups present in the surface layer is confirmed by the presence of a $\mathrm{O}=\mathrm{C}-\mathrm{NX} \mathrm{C} 1 \mathrm{~s}$ peak at $288.1 \mathrm{eV}$ (Figure 6.4C). The increase in the $\mathrm{O}-\mathrm{C}=\mathrm{O} \mathrm{C} 1 \mathrm{~s}$ peak at $289.0 \mathrm{eV}$ indicates that the aqueous EDC/NHS activation facilitates the enrichment of the films' surface with PAA when compared to samples directly after RIL. Covalent grafting of $\mathrm{PEG}_{113}-\mathrm{NH}_{2}$ to the EDC/NHS activated surface is successful, as is observed by the presence of a significant C-O-C C1s peak at $286.5 \mathrm{eV}$, which is the main $\mathrm{C} 1 \mathrm{~s}$ peak normally observed for PEG (Figure 6.4D). ${ }^{32}$ The $\mathrm{C} / \mathrm{O}$ ratio observed after PEG grafting is approximately 1.8 up to $\sin (\varphi)=0.7$. This change in $\mathrm{C} / \mathrm{O}$ ratio is in agreement with the expected change (for a homogeneous $P E G$ layer: $C / O=2.0$ ). The increase in carbon to oxygen ratio for higher $\sin (\varphi)$ was used to estimate the PEG layer thickness to be $7 \mathrm{~nm} \pm 1 \mathrm{~nm}$. This was confirmed by the presence of approximately 0.5 atom $\%$ nitrogen at $\varphi=45^{\circ}$ (escape depth $\sim 7 \mathrm{~nm}$ ) whereas a theoretical value of 0.3 atom $\%$ is expected for $\mathrm{PEG}_{113}-\mathrm{NH}_{2}$. The slight increase is ascribed to some residual NHS moieties within the investigated layer.

Our groups has reported thicknesses of approximately $4.9 \mathrm{~nm} \pm 0.3$ for $\mathrm{PEG}_{113}$ layers covalently grafted to trifluoroacetic acid activated PS- $b$-P $t$ BA surfaces by inverted $\mu \mathrm{CP}$ with PDMS stamps. ${ }^{15 \mathrm{~b}}$ This thickness exceeds the thickness for grafting reactions from solution. Apparently RIL activated surfaces are even more efficiently functionalized with $\mathrm{PEG}_{113}$ despite the enrichment of PS in the surface layer. The average grafting density $(\sigma)$ of PEG molecules per $\mathrm{nm}^{2}$ can be calculated according to equation $2:^{33}$

$\sigma=\frac{N_{A} d \rho_{d r y}}{M_{w}}$

where $N_{A}$ is Avogadro's number $\left(6.022 \times 10^{-23} \mathrm{~mol}^{-1}\right), d$ is the layer thickness, $\rho_{d r y}$ the dry PEG density $\left(1.06 \mathrm{~g} \mathrm{~cm}^{-3}\right)$ and $M_{w}$ the molar mass of PEG $\left(5000 \mathrm{~g} \mathrm{~mol}^{-1}\right)$.

According to this calculation the grafting density for $\mathrm{PEG}_{113}$ on EDC/NHS activated polymer films after RIL was $\sim 0.9$ chains $\mathrm{nm}^{-2}$. Compared to grafting densities of $\sim 2.9 \mathrm{PEG}_{11}\left(500 \mathrm{~g} \mathrm{~mol}^{-1}\right)$ molecules per $\mathrm{nm}^{2}$ reported by our group, ${ }^{34}$ an approximately 3 times increased PEG mass was found in this current study. ${ }^{35}$ Hence it 
is expected that the covalently grafted $\mathrm{PEG}_{113}$ film possesses good passivating capacities for the prevention of non-specific adsorption (NSA) of proteins.

After immersion of the PEG grafted samples in a BSA solution the XPS C1s spectrum did not significantly change (Figure 6.4E), which indicates that only a small amount of BSA physisorbed to the PEG passivated surface. This is even more obvious when the spectrum is compared with the spectrum obtained for a relatively thick layer of non-specifically physisorbed BSA on the bare films (Figure 6.4F). The C/O ratios of the PEG grafted sample before and after immersion in BSA solution did not differ significantly. In order to quantify the passivating capacity of the PEG layer, an equivalent BSA film thicknesses, $d_{\text {equivalent, }}$ was calculated for samples covalently functionalized with PEG and plain films, sample E and F, respectively (equation 3).

$d_{\text {equivalent }}=\frac{[N \%]_{\text {sample }}}{[N \%]_{B S A}} \times d_{\text {escape }}$

where $[N \%]_{\text {sample }}$ is the nitrogen atomic $\%$ related to BSA fouling and $[N \%]_{\mathrm{BSA}}$ is the theoretical nitrogen atomic \% based on the following BSA chemical composition, $\mathrm{C}_{2932} \mathrm{H}_{4619} \mathrm{O}_{898} \mathrm{~N}_{780} \mathrm{~S}_{39}{ }^{36}$ and $d_{\text {escape }}$ is the corresponding XPS photoelectron escape depth.

For the PEG functionalized sample and the plain PS- $b$-P $t$ BA film, $d_{\text {equivalent }}$ for the BSA layers of $0.7 \pm 0.31 \AA$ and $1.3 \pm 0.25 \mathrm{~nm}$, respectively, were calculated. Assuming a density of $1.28 \mathrm{~g} / \mathrm{cm}^{3}$ for the dried BSA layer, ${ }^{37}$ this reduction by $95 \%$ corresponds to a surface coverage of the PEG grafted sample of $9.1 \pm 2.9 \mathrm{ng} \mathrm{cm}^{-2}$. Current state of the art PEG-ylated surfaces have been reported to have a NSA of serum albumins of $\sim 1 \mathrm{ng} \mathrm{cm}^{-2}$. $32 \mathrm{a}, 38$

The occurrence of residual NSA may be in part attributed to the PDMS contamination that was found by XPS. On the other hand it is also known that NSA of proteins depends on factors such as PEG molar mass, grafting density, type of protein and protein solution concentration. ${ }^{36 a, 39}$ For a more sophisticated comparison of our RIL activated surfaces with other pegylated surfaces a more detailed study on the pegylation of these surfaces and NSA behavior is needed. 


\subsubsection{Fluorescence microscopic analyses of fluoresceinamine and MeO-PEG $\mathbf{1 1 3}^{-}$ $\mathrm{NH}_{2}$ functionalized $\mathrm{PS}_{2092}-b$-PtBA 1055 films}

The immobilization of functional molecules was further investigated by fluorescence microscopy. In particular, the lateral homogeneity of the attached fluorescence labels and the passivating capacity of the activated and subsequently PEG-passivated samples were tested as shown in Figure 6.6. In addition to the fluorescence microscopy images, the corresponding tapping mode AFM height images taken after RIL and subsequent functionalization are compared.

The AFM height images show the topographic line and pillar patterns imprinted with RIL for the films covalently grafted with fluoresceinamine and PEG, respectively. The surface modification did not alter the imprinted topographic structures. The presence of fluoresceinamine was observed as emission of light with $\lambda \sim 520 \mathrm{~nm}$ (green) in fluorescence microscopy. The observed intensity was lower compared to neat films thermolyzed in a nitrogen or air atmosphere or sample hydrolyzed with TFA, however, the homogeneity of the emission shows that the surface is functionalized evenly over large areas. The lower fluoresceinamine emission intensity observed when a PDMS stamp was in contact during RIL is ascribed to the presence of a hydrophobic PDMS contamination at the surface layer as was determined with XPS (data not shown).

The covalently immobilized PEG layers exhibited reduced non-specific adsorption of BSA, in full agreement with the XPS results discussed above. Physisorption of fluoresceinamine or PEG was excluded based on the analysis of control experiments in which the EDC/NHS activation of the carboxylic acid groups was omitted. Further control experiments showed measureable NSA of BSA on bare PDMS, which is in line with the tentative interpretation that the NSA on the PEG-ylated surfaces as quantified by XPS is indeed due to adsorption on PDMS. ${ }^{32 a, 38}$ Furthermore it was observed that the fluorescence emission of both fluoresceinamine or dye labeled BSA was not quenched by the presence of a relatively thick oligomeric PDMS solution deposited on top of the films. 


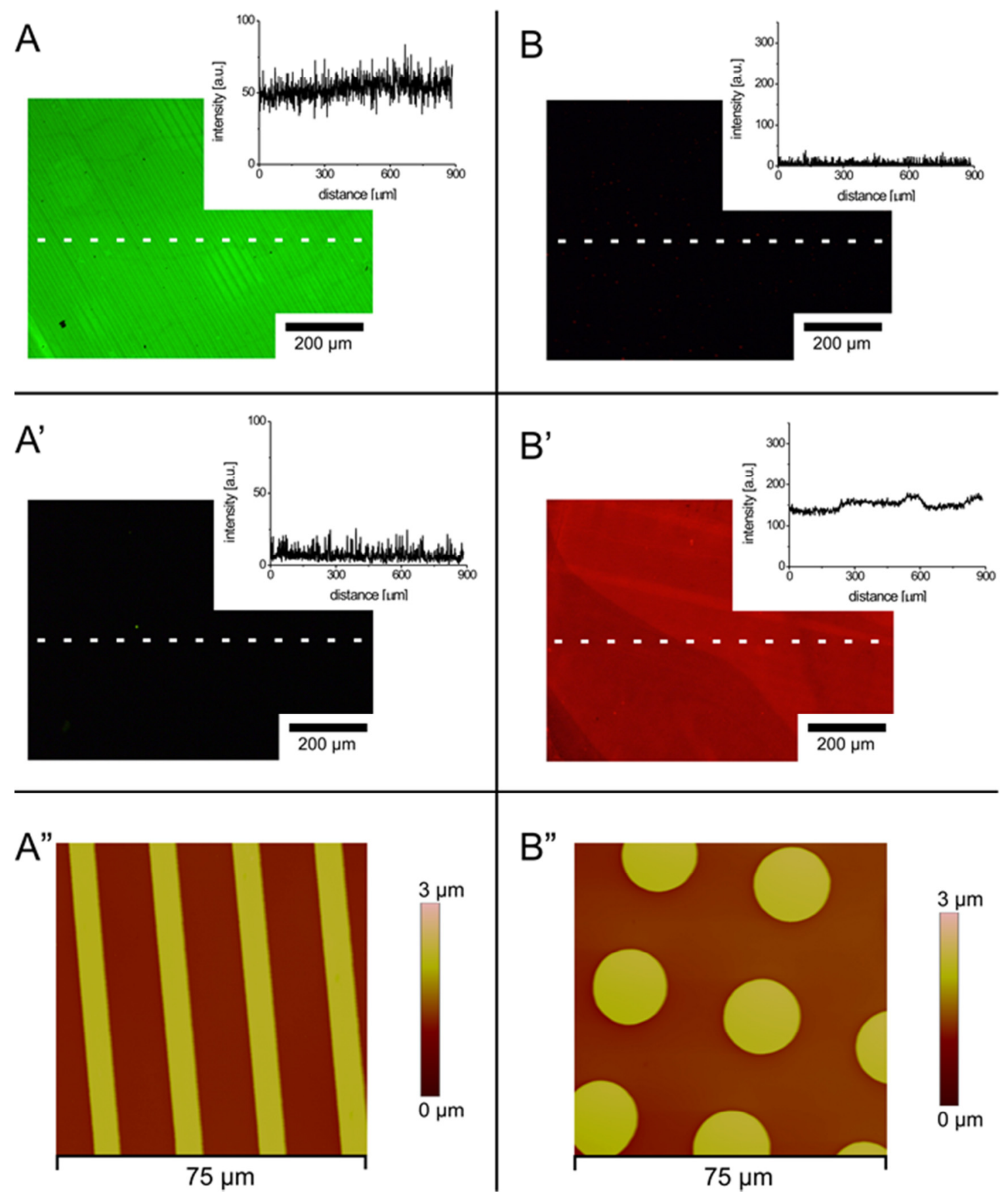

Figure 6.6: Top: Fluorescence microscopy images of RIL activated $P S_{2092}-b-P t B A_{1055}$ films after EDC/NHS mediated derivatization with (A) fluoresceinamine or (B) $P E G_{113}-N H_{2}$. The latter sample was immersed in a fluorescent dye labeled BSA solution prior to fluorescence microscopy analysis. Middle: Fluorescence microscopy images for the corresponding control experiments ( $A^{\prime}$ and $\left.B^{\prime}\right)$, in which the activation with EDC/NHS was omitted. The insets of the images are intensity cross-sections along the white dashed lines. Bottom: Tapping mode AFM height images for the corresponding samples shown at the top ( $A$ " and $B$ "). 
The results discussed above demonstrate the feasibility of RIL for the simultaneous one-step topographic pattering and chemical activation of PS- $b$-P $t$ BA films over large areas. The chemical reactions and physical mass transport processes occurring in the buried interface of block copolymer film and PDMS stamp resulted in differences in chemical reaction equilibria and significantly increased PEG surface coverages. The key factor in this new approach is the imprinting of the films at temperatures well above the thermal deprotection temperature of the tert-butyl ester to render the films carboxylic acid functional and structured over large areas.

Following the topographical structuring and homogeneous chemical functionalization using RIL, surface chemical patterns can be introduced in subsequent steps (not shown here). Our group has demonstrated the area-selective functionalization of topographically patterned PS- $b-\mathrm{P} t \mathrm{BA}$ films using the inverted $\mu \mathrm{CP}$ strategy developed by the Textor group. ${ }^{15 \mathrm{a}, \mathrm{b}}$ Here the PS- $b$-P $t \mathrm{BA}$ films were structured by an imprint lithography step, followed by a wet chemical deprotection of the tert-butyl ester using a strong organic acid. Since the use of acid is avoided in RIL, this method shows clear advantages. Another feasible approach comprises the removal of the remaining polymer film at the bottom of the trenches with an oxygen plasma $^{40}$ followed by selective chemical functionalization of the exposed silicon substrate in contrast to the existing carboxylic acid functionalized polymer matrix.

The feasibility of the thermochemical surface functionalization and subsequent bioconjugation of the block copolymer film platform described in this chapter stimulated us to start exploring scanning thermal lithography on PS- $b$-P $t$ BA films. These results will be described in Chapter 7 .

\subsection{Conclusions}

Reactive imprint lithography was introduced as a new one-step approach to topographically pattern and chemically activate $\mathrm{PS}_{2092}-b-\mathrm{P} t \mathrm{BA}_{1055}$ films. Tapping mode AFM analysis showed faithful pattern transfer from a silicon master to the polymer film. Transmission FTIR spectroscopy confirmed a near quantitative conversion of tert-butyl ester groups to carboxylic acid and anhydride groups and revealed that intramolecular anhydride formation was greatly inhibited by the presence of a PDMS stamp compared to thermolysis of free surfaces. Angle resolved XPS provided insight in the polymer films' surface chemical composition before and after RIL as well as after the wet chemical grafting reactions. PEG layers were covalently grafted to RIL activated films with a grafting density of $\sim 0.9$ chains $\mathrm{nm}^{-2}$ and a layer thickness of $\sim 7 \pm 1 \mathrm{~nm}$. These PEG layers reduced BSA fouling by $95 \%$ 
to $9.1 \pm 2.9 \mathrm{ng} \mathrm{cm}^{-2}$. RIL was shown to be a promising complementary approach for the preparation of bio-reactive polymer surfaces with topographical patterns in a onestep process.

\subsection{Experimental}

Materials: $\mathrm{PS}_{2092}-b-\mathrm{P} t \mathrm{BA}_{1055}$ (the numbers in subscript denote the degree of polymerization for each block respectively) was purchased from Polymer Source Inc. (Dorval, Canada). The total molar mass of this polymer is $352 \mathrm{~kg} / \mathrm{mol}$ with a polydispersity index of 1.10. Fluoresceinamine isomer $\mathrm{I}\left(\lambda_{\max }=496 \mathrm{~nm}\right)$, trifluoroacetic acid (TFA), N-hydroxysuccinimide (NHS) and 1-ethyl-3-(3dimethylaminopropyl)carbodiimide hydrochloride (EDC) were obtained from Sigma-Aldrich (St. Louis, MO, USA). Amino end-functionalized poly(ethylene glycol) $\left(\mathrm{MeO}-\mathrm{PEG}_{113}-\mathrm{NH}_{2}\right.$, molar mass $\left.=5 \mathrm{~kg} / \mathrm{mol}\right)$ was bought from Iris Biotech $\mathrm{GmbH}$ (Marktredwitz, Germany). Toluene and ethanol were purchased from Biosolve (Valkenswaard, The Netherlands) and phosphate buffered saline solution was obtained from B. Braun (Melsungen, Germany). Bovine serum albumin (BSA) Alexa Fluor ${ }^{\circledR 594}$ conjugate was bought from Molecular Probes/Invitrogen Co. (Carlsbad, CA, USA). All compounds were used without further purification. Milli-Q water was produced by a Milipore Synergy system (Billereca, MA, USA).

Film preparation: $\mathrm{PS}_{2092}-b-\mathrm{P} t \mathrm{BA}_{1055}$ polymer films were prepared by spin-coating polymer solutions (typically $10 \mathrm{wt} \%$ ) in toluene onto precleaned $1 \mathrm{~cm} \times 1 \mathrm{~cm}$ silicon substrates (CZ, type P, boron, «100〉, thickness $=525 \mu \mathrm{m}$, OKMETIC, Vantaa, Finland) at $3000 \mathrm{rpm}$ for 30 seconds (P6700 spin coater, Specialty Coating Systems Inc., Indianapolis, IN, USA). Silicon substrates were cleaned in piranha solution $\left(1: 3(\mathrm{v} / \mathrm{v})\right.$ solution of $30 \% \mathrm{H}_{2} \mathrm{O}_{2}$ and concentrated $\left.\mathrm{H}_{2} \mathrm{SO}_{4}\right)$ for 10 minutes, rinsed with copious amounts of MilliQ water and ethanol followed by spin drying, prior to use.

Caution: Piranha solution should be handled with extreme caution; it has been reported to detonate unexpectedly. All spin-coated films were annealed at $135{ }^{\circ} \mathrm{C}$ in vacuum for 12 hours.

A Plasmos SD 2002 ellipsometer (Munich, Germany) at fixed wavelength (632.8 nm) and angle $\left(70^{\circ}\right)$ was used to determine the mean film thickness. A refractive index of 1.513 was used for the block copolymer film ${ }^{15 b, 25}$ in the thickness calculations. The mean film thickness was determined to be $1120 \mathrm{~nm}$ with a standard deviation of $4 \mathrm{~nm}$. Mold preparation: PDMS stamps $(\sim 1 \mathrm{~cm} \times 1 \mathrm{~cm})$ were prepared from an elastomeric PDMS kit, containing Sylgard-184A (elastomer) and Sylgard-184B (curing agent) (Dow Corning, Midland, MI, USA). Typically 10:1 (w/w) elastomer to curing agent mixtures were degassed by applying a vacuum for 20 minutes in two subsequent 
pumping cycles before pouring them carefully over a trichloro(tridecafluorooctyl)silane anti-adhesive coated silicon master, either with or without a topographical pattern. Following two more degassing cycles the PDMS was cured at $60{ }^{\circ} \mathrm{C}$ for 12 hours. After cooling to room temperature the resulting PDMS stamps were carefully removed from the silicon master and stored at room temperature in a closed poly(styrene) box. Prior to use the PDMS stamps were sonicated for 20 minutes in ethanol, rinsed with ethanol and dried under a nitrogen stream.

Reactive imprint lithography (RIL): Polymer films on silicon substrates were imprinted in a temperature controlled hydraulic press (Specac, London, UK) using silicon or PDMS stamps typically at $10 \mathrm{MPa}$ for different temperature profiles and molding times, e.g. $150{ }^{\circ} \mathrm{C}$ for 40 minutes followed by $230{ }^{\circ} \mathrm{C}$ for 5 minutes. Demolding was done at temperatures around $70^{\circ} \mathrm{C}$.

Atomic force microscopy (AFM): AFM measurements were carried out with a Dimension D3100 AFM operated with a NanoScope IVa controller (Digital Instruments/Veeco, Santa Barbara, CA, USA). Tapping mode AFM height images were recorded with silicon cantilevers/tips (OTESPA, Veeco, Santa Barbara, CA) in air. The system operating frequency was typically $10 \%$ lower than the natural resonance frequency of the cantilever in air, the free amplitude was kept constant, while the set point amplitude was approximately $86 \%$ of the free amplitude $(\sim 2.0 \mathrm{~V})$. Immobilization of (bio)molecules on RIL activated polymer films: Following RIL of polymer films the in situ formed carboxylic acid groups were activated with EDC/NHS (200 mM and $250 \mathrm{mM}$, respectively, in PBS). After 1 hour EDC/NHS activation the samples were rinsed 3 times with PBS and immersed for 1 hour in either a $\mathrm{MeO}-\mathrm{PEG}_{113}-\mathrm{NH}_{2}$ or fluoresceinamine solution in PBS (concentration $\sim 1 \times 10^{-4} \mathrm{M}$ ). Fluoresceinamine modified films were extensively rinsed with Milli-Q water prior to drying in a nitrogen stream. PEG-ylated samples were rinsed 3 times with PBS. To test the quality of the PEG layers, the samples were placed in a BSA Alexa Fluor ${ }^{\circledR}$ conjugate solution (concentration $\sim 1 \times 10^{-5} \mathrm{M}$ ). Subsequently, the films were rinsed with copious amounts of Milli-Q water and dried in a nitrogen stream.

Fourier transform infrared (FTIR) spectroscopy: Transmission mode FTIR spectra (spectral resolution $4 \mathrm{~cm}^{-1}$, 1024 scans) were collected with a BIO-RAD model FTS575C FTIR spectrometer equipped with a liquid nitrogen-cooled cryogenic mercury cadmium telluride detector (Hercules, CA, USA). Silicon substrates were used for background spectra collection immediately after they were piranha cleaned for 10 minutes, rinsed with copious amounts of MilliQ water and ethanol followed by dry blowing in a nitrogen stream. 
Angle dependent X-ray photoelectron spectroscopy: XPS spectra were recorded on a PHI Quantera XPS microprobe (Physical Electronics Inc., Chanhassen, MN, USA) using a monochromatic X-ray beam (Al Ka monochromatic at $1486.6 \mathrm{eV}, 100 \mu \mathrm{m}$ diameter, $25 \mathrm{~W})$ at variable take-off angles $(\varphi)$ between $5^{\circ}$ and $70^{\circ}$ immediately after sample preparation. Charging of samples was corrected by shifting the peak positions relative to the position of neutral carbon at $285.0 \mathrm{eV}(\mathrm{C} 1 \mathrm{~s})$. Atomic concentrations were determined by numerical integration of the relative peak areas using the Multipak software with supplied atomic sensitivity factors relative to F1s (C1s: 0.314; O1s: 0.733; Si2p: 0.368; N1s: 0.499). ${ }^{41}$ Peak fitting of the C1s spectra was done using the same software. Peak positions were restricted to $\pm 0.1 \mathrm{eV}$ of the literature of the corresponding peak position, while all other parameters were floatable.

Fluorescence microscopy: Fluorescence microscopy images of dried polymer films were taken directly after preparation of the samples with an Olympus IX71 fluorescence microscope. For the excitation of fluoresceinamine an U-MWB-2 filter cube was used, while for the excitation of the Alexa Fluor®594 labeled BSA and U-MWB-2 filter cube combined with a DM570 dichroic mirror and NA590 barrier filter was used. Typical sample exposure times were 50 to $100 \mathrm{~ms}$.

\subsection{References and notes}

1 a) Mrksich, M. Chem. Soc. Rev. 2000, 29, 267. b) Wong, J. Y.; Leach, J. B.; Brown, X. Q. Surf. Sci. 2004, 570, 119. c) Lussi, J. W.; Michel, R.; Reviakine, I.; Falconnet, D.; Goessl, A.; Csucs, G.; Hubbell, J. A.; Textor, M. Prog. Surf. Sci. 2004, 76, 55.

2 a) Hatakeyama, H.; Kikuchi, A.; Yamato, M.; Okano, T. Biomaterials 2007, 28, 3632. b) Tan, W.; Desai, T. A. Biomaterials 2004, 25, 1355. c) Park, T. H.; Shuler, M. L. Biotechnol. Prog. 2003, 19, 243. d) Khademhosseini, A.; Langer, R. Biomaterials 2007, 28, 5087.

3 a) Jonkheijm, P.; Weinrich, D.; Schröder, H.; Niemeyer, C. M.; Waldmann, H. Angew. Chem. Int. Ed. 2008, 47, 9618. b) Phizicky, E.; Bastiaens, P. I. H.; Zhu, H.; Snyder, M.; Fields, S. Nature 2003, 422, 208. c) MacBeath, G.; Schreiber, S. L. Science 2000, 289, 1760. d) Wells, M.; Crooks, R. M. J. Am. Chem. Soc. 1996, 118, 3988. e) La Fratta, C. M.; Walt, D. R. Chem. Rev. 2008, 108, 614.

Embrechts, A.; Feng, C. L.; Bredebusch, I.; Rommel, C. E.; Schnekenburger, J.; Vancso, G. J.; Schönherr, H. in Surface Design: Applications in Bioscience and Nanotechnology, Eds: R. Förch, R.; Schönherr, H.; Jenkins, A. T. A., Wiley-VCH, 2009, p. 233.

5 a) Griesser, T.; Adams, J.; Wappel, J.; Kern, W.; Leggett, G. J.; Trimmel, G. Langmuir 2008, 24, 12420. b) Larsson, A.; Du, C.; Liedberg, B. Biomacromolecules 2007, 8, 3511. c) Falconnet, D.; Koenig, A.; Assi, T.; Textor, M. Adv. Funct. Mater. 2004, 14, 749. d) Buxboim, A.; BarDagan, M.; Frydman, V.; Zbaida, D.; Morpurgo, M.; Bar-Ziv, R. Small 2007, 3, 500.

a) Gates, D. B.; Xu, Q.; Stewart, M.; Ryan, D.; Willson, C. G.; Whitesides, G. M. Chem. Rev. 2005, 105, 1171. b) Dusseiller, M. R.; Schlaepfer, D.; Koch, M.; Kroschewski, R.; 
Textor, M. Biomaterials 2005, 26, 5917. c) Delamarche, E.; Donzel, C.; Kamounah, F. S.; Wolf, H.; Geissler, M.; Stutz, R.; Schmidt-Winkel, P.; Michel, B.; Mathieu, H. J.; Schaumburg, K. Langmuir 2003, 19, 8749. d) Feng, C. L.; Vancso, G. J.; Schönherr, H. Adv. Funct. Mater. 2006, $16,1306$.

7 a) Johansson, F.; Carlberg, P.; Danielsen, N.; Montelius, L.; Kanje, M. Biomaterials 2006, 27, 1251. b) Zhang, G. M.; Zhang, J.; Xie, G. Y.; Liu, Z. F.; Shao, H. B. Small 2006, 2, 1440. c) Falconnet, D.; Pasqui, D.; Park, S.; Eckert, R.; Schift, H.; Gobrecht, J.; Barbucci, R.; Textor, M.; Nano lett. 2004, 4, 1909. d) Truskett, V. N.; Watts, M. P. C. Trends Biotechnol. 2006, 24, 312.

a) Christman, K. L.; Schopf, E.; Broyer, R. M.; Li, R. C.; Chen, Y.; Maynard, H. D. J. Am. Chem. Soc. 2009, 131, 521. b) Hong, Y.; Krsko, P.; Libera, M. Langmuir 2004, 20, 11123. c) Glass, R.; Arnold, M.; Blümmel, J.; Küller, A.; Möller, M.; Spatz, J. P. Adv. Funct. Mater. 2003, 13, 569.

a) García, R.; Martinez, R. V.; Martinez, J. Chem. Soc. Rev. 2006, 35, 29. b) Benetti, E. M.; Chung, H. J.; Vancso, G. J. Macromol. Rapid Comm. 2009, 30, 411. c) Sun, S.; Leggett, G. J. Nano Lett. 2002, 2, 1223. d) Wouters, D.; Schubert, U. S. Angew. Chem. Int. Ed. 2004, 43, 2480.

10 a) Arnold, M.; Cavalcanti-Adam, E. A.; Glass, R.; Blümmel, J.; Eck, W.; Kantlehner, M.; Kessler, H.; Spatz, J. P. Chem. Phys. Chem. 2004, 5, 383. b) Dalby, M. J.; Childs, S.; Riehle, M. O.; Johnstone, H. J. H.; Affrossman, S.; Curtis, A. S. G. Biomaterials 2003, 24, 927. c) Shipway, A. N.; Katz, E.; Willner, I. Chem. Phys. Chem. 2000, 1, 18. d) Walter, N.; Selhuber, C.; Kessler, H.; Spatz, J. P. Nano Lett. 2006, 6, 398.

11 Böltau, M.; Walheim, S.; Mlynek, J.; Krausch, G.; Steiner, U. Nature 1998, 391, 877.

12 Zhang, J.; Venkataramani, S.; Xu, H.; Song, Y. K.; Song, H. K.; Palmore, G. T. R.; Fallon, J.; Nurmikko, A. V. Biomaterials 2006, 27, 5734.

13 Mrksich, M.; Chen, C. S.; Xia, Y.; Dike, L. E.; Ingber, D. E.; Whitesides, G. M. P. Natl. Acad. Sci USA 1996, 93, 10775.

14 Pan, F.; Wang, P.; Lee, K.; Wu, A.; Turro, N. J.; Koberstein, J. T. Langmuir 2005, 21, 3605.

15 a) Dusseiller, M. R.; Schlaepfer, D.; Koch, M.; Kroschewski, R.; Textor, M. Biomaterials 2005, 26, 5917. b) Embrechts, A.; Feng, C. L.; Mills, C. A.; Lee, M.; Bredebusch, I.; Schnekenburger, J.; Domschke, W.; Vancso, G. J.; Schönherr, H. Langmuir 2008, 24, 8841. c) Charest, J. L.; Eliason, M. T.; García, A. J.; King, W. P. Biomaterials 2006, 27, 2487.

16 Schift, H. J. Vac. Sci. Technol. B 2008, 26, 458.

17 Fredin, N. J.; Broderick, A. H.; Buck, M. E.; Lynn, D. M. Biomacromolecules 2009, $10,994$.

18 a) Böker, A.; Reihs, K.; Wang, J.; Stadler, R.; Ober, C. K. Macromolecules 2000, 33, 1310. b) Böker, A.; Herweg, T.; Reihs, K. Macromolecules 2002, 35, 4929.

19 a) La, Y. H.; Edwards, E. W.; Park, S. M.; Nealey, P. F. Nano Lett. 2005, 5, 1379. b) La, Y. H.; Stoykovich, M. P.; Park, S.M.; Nealey, P. F. Chem. Mater. 2007, 19, 4538.

Szoszkiewicz, R.; Okada, T.; Jones, S. C.; Li, T. D.; King, W. P.; Marder, S.R.; Riedo, E. Nano Lett. 2007, 7, 1064.

21 Duvigneau, J.; Schönherr, H.; Vancso, G. J. Langmuir 2008, 24, 10825.

22 Qin, D.; Xia, Y.; Whitesides, G. M. Adv. Mater. 1996, 8, 917.

23 a) Odom, T. W.; Love, J. C.; Wolfe, D. B.; Paul, K. E.; Whitesides, G. M. Langmuir 2002, 18, 5314. b) Schmid, H.; Michel, B. Macromolecules 2000, 33, 3042. c) Makamba, H.; Kim, J. H.; 
Lim, K.; Park, N.; Hahn, J. H. Electrophoresis 2003, 24, 3607. d) Muzzalupo, R.; Ranieri, G. A.; Golemme, G.; Drioli, E. J. Appl. Pol. Sci. 1999, 74, 1119.

24 Brandrup, J.; Immergut, E. H. in Polymer Handbook, 3rd ed., John Wiley \& Sons: New York, 1989.

25 Feng, C. L.; Embrechts, A.; Vancso, G. J.; Schönherr, H. Eur. Polym. J. 2006, 42, 1954.

26 Assuming a linear thermal expansion in the lateral direction and neglecting cooling, the maximum thermal expansion is calculated as follows: $\Delta \mathrm{L} / \mathrm{L}_{0}=\alpha_{\mathrm{L}} \times \Delta \mathrm{T}$ in which $\alpha_{\mathrm{L}}=200 \times 10^{-6}$ $\mathrm{K}^{-1}, \Delta \mathrm{T}=210 \mathrm{~K}$ and $\mathrm{L}_{0}=19.2 \mu \mathrm{m}$. Hence, the increase in periodicity was calculated to be approximately $0.8 \mu \mathrm{m} . \alpha_{\mathrm{L}}$ was estimated from: Schroeder, M. J.; Roland, C. M. Macromolecules 2002, 35, 2676.

27 a) Favre, E.; Schaetzel, P.; Nguygen, Q. T.; Clément, R.; Néel, J. J. Membrane Sci. 1994, 92, 169. b) Park, Y. I.; Yeom, C. K.; Kim, B. S.; Suh, J. K.; Hong, J. S.; Lee, J. M.; Joo, H. J. Desalination 2008, 233, 303.

28 Liu, Z.; Pappacena, K.; Cerise, J.; Kim, J.; Durning, C. J.; O’Shaughnessy, B.; Levicky, R. Nano Lett. 2002, 2, 219.

29 Feng, C. L.; Vancso, G. J.; Schönherr, H. Langmuir 2005, 21, 2356.

30 a) Quist, A. P.; Pavlovic, E.; Oscarsson, S. Anal. Bioanal. Chem. 2005, 381, 591. b) Thibault, C.; Séverac, C.; Mingotaud, A.; Vieu, C.; Mauzac, M. Langmuir 2007, 23, 10706.

31 a) Hamann, P. R.; Hinman, L. M.; Beyer, C. F.; Lindh, D.; Upeslacis, J.; Flowers, D. A.; Bernstein, I. Bioconjugate Chem. 2002, 13, 40. b) Wissink, M. J. B.; Beernink, R.; Pieper, J. S.; Poot, A. A.; Engbers, G. H. M.; Beugeling, T.; van Aken, W. G.; Feijen, J. J. Biomaterials 2001, 22, 151.

32 a) Sofia, S. J.; Premnath, V.; Merrill, E. W. Macromolecules 1998, 31, 5059. b) Kingshott, P.; McArthur, S.; Thissen, H.; Castner, D.G.; Griesser, H. J. Biomaterials 2002, 23, 4775.

33 Mougin, K.; Lawrence, M.; Fernandez, E. J.; Hillier, A. C. Langmuir 2004, 20, 4302.

34 Feng, C. L.; Embrechts, A.; Bredebusch, I.; Bouma, A.; Schnekenburger, J.; García-Parajó, M.; Domschke, W.; Vancso, G. J.; Schönherr, H. Eur. Polym. J. 2007, 43, 2177.

35 The lower grafting density is expected for grafting of longer molecules: Yang, Z.; Galloway, J. A.; Yu, H. Langmuir 1999, 15, 8405.

36 Zhang, M.; Murakami, T.; Ajima, K.; Tsuchida, K.; Sandanayaka, A. S. D.; Ito, O.; Iijima, S.; Yudasaka, M. P. Natl. Acad. Sci USA 2008, 105, 14773.

37 Bolton, B. A.; Scherer, J. R.; J. Phys. Chem. 1989, 93, 7635.

38 a) Huang, N. P.; Michel, R.; Vörös, J.; Textor, M.; Hofer, R.; Rossi, A.; Elbert, D. L.; Hubbell, J. A.; Spencer, N. D. Langmuir 2001, 17, 489. b) Pasche, S.; De Paul, S.; Vörös, J.; Spencer, N. D.; Textor, M. Langmuir 2003, 19, 9216. c) Sato, Y.; Yoshioka, K.; Tanaka, M.; Murakami, T.; Ishida, M.N.; Niwa, O. Chem. Commun. 2008, 4909.

39 Kenausis, G. L.; Vörös, J.; Elbert, D. L.; Huang, N.; Hofer, R.; Ruiz-Taylor, L.; Textor, M.; Hubbell, J. A.; Spencer, N. D. J. Phys. Chem. B 2000, 104, 3298.

40 Bennett, R. D.; Xiong, G. Y.; Ren, Z. F.; Cohen, R. E. Chem. Mater. 2004, 16, 5589.

41 Zhao, J.; Garza, E. G.; Lam, K.; Jones, C. M. Appl. Surf. Sci. 2000, 158, 246. 


\section{Chapter 7 \\ Scanning Thermal Lithography of PtBA Block Copolymer Films for Bioconjugation}

In this chapter scanning thermal lithography (SThL) in conjugation with block copolymer film platforms is introduced. The local thermal activation of thin polymer films for area-selective surface chemical modification on micrometer and nanometer length scales is described in detail. The thermally induced activation of tert-butyl ester moieties in poly(styrene)-block-poly(tert-butyl acrylate) (PS- $b$-P $t$ BA) block copolymer films leads to the formation of pending carboxylic acid groups, which are among the versatile functionalities for subsequent bioconjugation. From FTIR spectroscopic analysis the apparent activation energy $\left(E_{a}\right)$ for the tert-butyl ester deprotection in thin films was calculated to be $93 \pm 12 \mathrm{~kJ} \mathrm{~mol}^{-1}$, which is in good agreement with values reported for the bulk. The availability of the deprotected carboxylic acid groups in subsequent wet chemical grafting reactions on neat thermolyzed films was confirmed by covalently immobilizing fluoresceinamine and amino-endfunctionalized poly(ethylene glycol) ( $\left.\mathrm{PEG}-\mathrm{NH}_{2}\right)$ using established 1-ethyl3-(3-dimethylamino-propyl)carbodiimide hydrochloride / $\mathrm{N}$-hydroxysuccinimide (EDC/NHS) chemistry. Local thermal deprotection on $\mu \mathrm{m}$ and sub- $\mu \mathrm{m}$ length scales was achieved by SThL using an AFM equipped with heatable probe tips. Passivating PEG and fluoresceinamine layers were selectively covalently coupled to locally deprotected areas as small as $370 \mathrm{~nm} \times 580 \mathrm{~nm}$.

\footnotetext{
* Parts of this chapter have been published in: Duvigneau, J.; Schönherr, H.; Vancso, G. J. Langmuir 2008, 24, 10825.
} 


\subsection{Introduction}

Polymer thin films comprise promising platforms for surface structuring and patterning in diverse applications. ${ }^{1}$ The areas of interest range from advanced data storage $^{2}$ and photonic crystals ${ }^{3}$ to the development of substrates with tailored biointerfaces for cell-surface interaction studies ${ }^{4}$ or advanced (bio)sensors. ${ }^{5}$

In the context of surface structuring and patterning, for instance employing imprint lithographic approaches, the glass transition temperature $\left(T_{g}\right)$ of polymers and polymer films is of crucial importance. While polymers can be easily shaped at temperatures above $T_{g}$, they remain dimensionally stable at lower temperatures. This well-established macroscale concept has been exploited in nanoimprint lithography to fabricate structures in amorphous polymers down to $10 \mathrm{~nm}$ length scales. ${ }^{6}$ Provided that a suitable master is available (fabricated e.g. by electron beam lithography or focused ion beam milling), such structures can be produced in a massively parallel fashion.

When polymers are subjected to heat and pressure during imprinting, chemical transformations of the material are undesirable. By contrast, thermally induced reactions of polymers represent a class of yet largely unexplored reactions in terms of controlled surface modification and structuring. The few known examples at the start of this project have been introduced in Chapter 1 and Chapter $6 .{ }^{7,8}$ Simultaneous with our attempts described in this chapter, King and coworkers ${ }^{9,10}$ reported on the introduction of scanning thermal lithography (SThL). Since heat transport at small length scales does not suffer from limitations of classic lithography (given by the diffraction limit) or various drawbacks of scanning probe lithographies (SPL), SThL is expected to provide an avenue to highly defined structures.

Advanced SPL approaches that are applicable to soft condensed matter utilize, e.g., the transport of molecules from an atomic force microscopy (AFM) tip to a suitable substrate in dip pen nanolithography (DPN) ${ }^{11}$ or local photochemistry exploited in conjunction with scanning near-field optical microscopy (SNOM). ${ }^{12}$ These methods possess their particular areas of applicability and their own limitations. Although these techniques allow one to approach patterning down to or close to (macro)molecular length scales, molecular diffusion ${ }^{13}$ and the inefficient intensity of the near-field for very small apertured SNOM probes ${ }^{14}$ limit the attainable resolution.

As mentioned, thermally induced reactions have not been studied in detail for the purpose of patterning surfaces with high resolution. In addition to a localized heat source, a suitable platform with efficient surface chemistry is required. The recently introduced block copolymer film platform based on poly(styrene)-block-poly(tertbutyl acrylate), $\mathrm{PS}_{\mathrm{n}}-b-\mathrm{P} t \mathrm{BA}_{\mathrm{m}}$ meets the requirements for the immobilization of interesting (bio)molecules for the fabrication of tailored biointerfaces. ${ }^{15}$ The internal structure of the block copolymer films of $\mathrm{PS}_{690^{-}}-b-\mathrm{P} t \mathrm{BA}_{1210}$ with microphase separated 
PS cylinders and a skin layer of reactive PtBA exposed at the film surface can be exploited to obtain very robust architectures that show excellent stability under a broad range of processing conditions. The process comprises the acid-catalyzed deprotection of the $t \mathrm{BA}$ groups, NHS activation and covalent grafting of various functionalities. Using new concepts of soft lithography, patterns down to the $300 \mathrm{~nm}$ size range were also fabricated. ${ }^{16}$ In addition, nanoscale patterns are in principle accessible, ${ }^{17}$ however, the as-prepared and annealed films exhibit, as mentioned, a continuous skin layer of $\mathrm{P} t \mathrm{BA}$.

In addition to its instability in presence of acid, ${ }^{18}$ the $t$ BA ester group is known to undergo thermal reactions (see also Chapter 6). Upon heating isobutylene (gas) is liberated, resulting in carboxylic acid moieties. ${ }^{19}$ In the case of PtBA, the poly(acrylic acid) obtained can be further converted to the anhydride polymer by an intramolecular cyclization reaction (Figure 7.1). Both carboxylic acid and anhydride functionalities are versatile groups for further modification with a range of biologically interesting molecules.

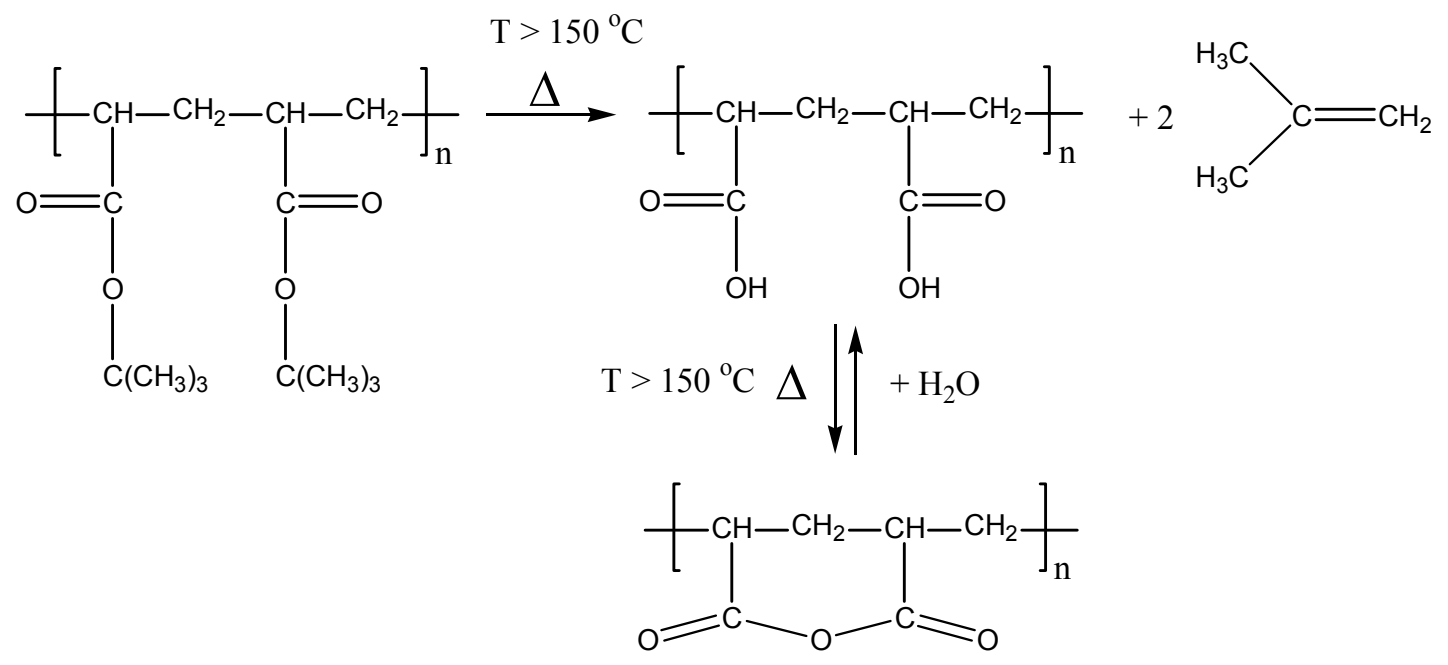

Figure 7.1: Thermal deprotection reaction of the PtBA block in $P S_{n}-b-P t B A_{m}$ block copolymers. The intramolecular cyclization step is limited in yield due to statistical reasons to $87 \% .^{20}$

This chapter describes the local thermal activation of thin block copolymer films by SThL for area-selective surface chemical modification down to sub- $\mu \mathrm{m}$ length scales. Based on the thorough investigation of the mechanism and kinetics of the thermally induced activation of $t \mathrm{BA}$ ester moieties in PS- $b$-P $t$ BA block copolymer films and the results described in Chapter 6, the basis for nanoscale local deprotection using a scanning thermal microscope was established. 


\subsection{Results and discussion}

The thermal deprotection reaction of the $t \mathrm{BA}$ ester groups in the $\mathrm{P} t \mathrm{BA}$ phase of thin films of $\mathrm{PS}_{\mathrm{n}}-b-\mathrm{P} t \mathrm{BA}_{\mathrm{m}}$ block copolymers on silicon was first investigated in detail in the temperature range of $200{ }^{\circ} \mathrm{C}$ to $250{ }^{\circ} \mathrm{C}$. Thereby, the possible impact of thin film confinement on the thermolysis mechanism and the corresponding reaction kinetics and activation energy was investigated. The subsequently studied chemical activation of the carboxylic acid groups generated in the thermolysis and covalent immobilization of $\alpha$-amines forms the basis for the targeted local thermal activation by SThL approaches and following functionalization.

\subsubsection{Mechanism of thermolysis}

It is well established that bulk $\mathrm{P} t \mathrm{BA}$ is converted at temperatures higher than $150{ }^{\circ} \mathrm{C}$ to poly(acrylic anhydride) $(\mathrm{PAnH})$ with the formation of poly(acylic acid) (PAA) as an intermediate step (Figure 7.1, see also Chapter 6). ${ }^{8,19,21-23}$ Isothermal TGA data of bulk PS- $b$-P $t$ BA samples confirmed that the thermal bulk conversion of $\mathrm{P} t \mathrm{BA}$ to $\mathrm{PAnH}$ and PAA goes to completion to within the experimental error of the experiment for temperatures between $230{ }^{\circ} \mathrm{C}$ and $250{ }^{\circ} \mathrm{C}$ (Figure 7.2). Based on the block copolymer composition, the expected mass loss due to the thermolysis of the $t$ BA ester is $28 \mathrm{wt} \%$ (upper dashed line) and the subsequent loss of water in the second step contributes another $5 \mathrm{wt} \%$ (lower dashed line).

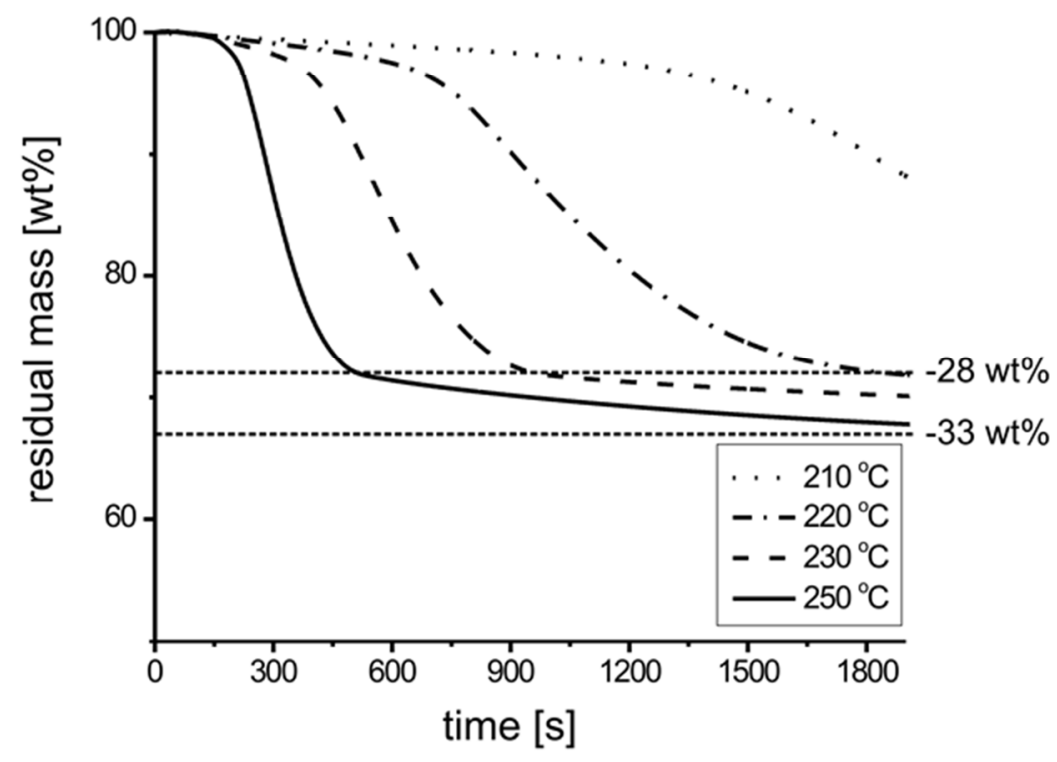

Figure 7.2: Isothermal thermo gravimetric analysis (TGA) data of $P S_{644}-b-P t B A_{938}$ for thermolysis temperatures between $230{ }^{\circ} \mathrm{C}$ and $250{ }^{\circ} \mathrm{C}$ showed that the bulk thermolysis reaches completion within 30 minutes according to the mechanism shown in Figure 7.1. 
Mass losses found for isothermal thermolysis at $230{ }^{\circ} \mathrm{C}$ and $250{ }^{\circ} \mathrm{C}$ were $30 \mathrm{wt} \%$ and $32 \mathrm{wt} \%$, respectively. Therefore, this temperature region was investigated with FTIR spectroscopy to confirm the presence and disappearance of characteristic functional groups in thin PS- $b$-P $t$ BA thin films within each step of the mechanism as proposed in Figure 7.1.

Figure 7.3 shows FTIR spectra of block copolymer films before and after thermolysis at $240{ }^{\circ} \mathrm{C}$ (compare Figure 6.3). Before thermolysis, both the $v_{\mathrm{C}=\mathrm{O}}$ at $1733 \mathrm{~cm}^{-1}$ (dashed line), characteristic for ester carbonyl absorbances, and the $\delta_{\mathrm{C}-\mathrm{H}}$ at $1368 \mathrm{~cm}^{-1}$ and $1392 \mathrm{~cm}^{-1}$, characteristic for methyl bending absorbances, are clearly visible. Upon thermolysis the latter bands disappeared completely, which agrees with the complete conversion of the $t \mathrm{BA}$ ester to acrylic acid as can be seen in the spectra for 260 seconds and 540 seconds thermolysis at $240{ }^{\circ} \mathrm{C}$. Furthermore for the spectrum obtained at 260 seconds a shift in the carbonyl absorbances to $1709 \mathrm{~cm}^{-1}$ and to $1755 \mathrm{~cm}^{-1}$ and $1804 \mathrm{~cm}^{-1}$ was observed, which corresponds to the carbonyl absorbances of carboxylic acid and anhydride groups, respectively. For longer thermolysis times, the $v_{\mathrm{C}=\mathrm{O}}$ at $1709 \mathrm{~cm}^{-1}$ showed significantly reduced absorbances. Using spectral deconvolution a conversion of PAA to PAnH of $60 \%$ was expected for long reaction times. This value may represent an underestimate, since the anhydride may react back to the carboxylic acid form in humid air and since decarboxylation may already play a role, albeit limited, under these conditions (see also below). From this, we conclude that the thermal activation mechanism of $\mathrm{PS}_{644}-b-\mathrm{P} t \mathrm{BA}_{938}$ polymer thin films is consistent with the mechanism shown in Figure 7.1 for thermal activation of bulk PtBA. The presence of the PS block does not alter the mechanism. This result is in agreement with data reported by Nealey et al. obtained for thicker PS- $b$-P $t$ BA films on gold substrates at lower thermolysis temperatures. ${ }^{8}$ 


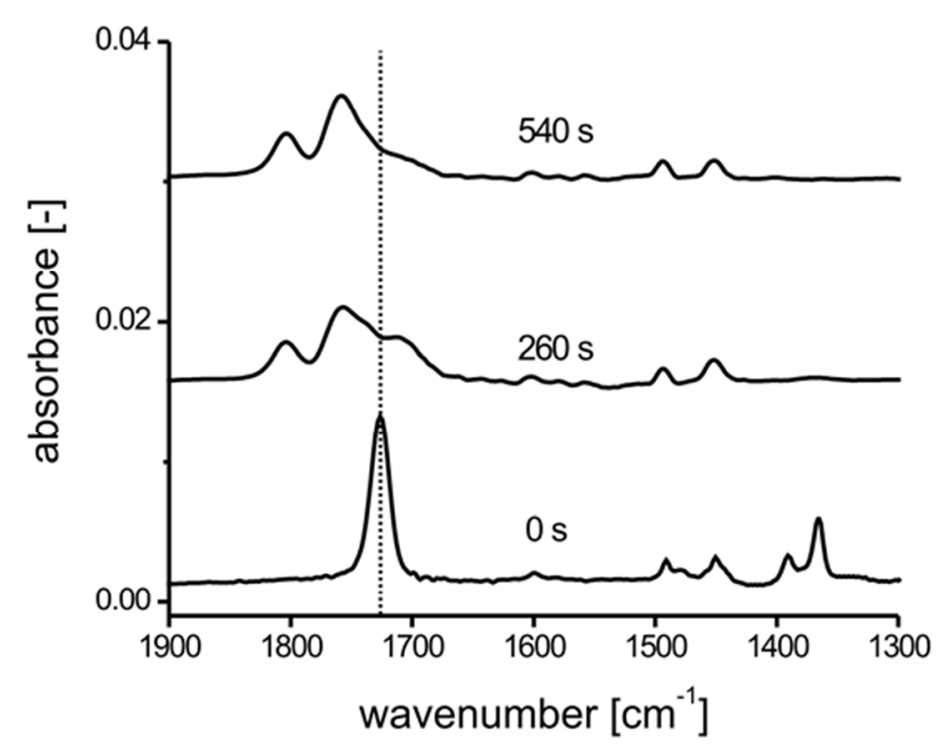

Figure 7.3: Transmission FTIR spectra of $P_{644}-b-P t B A_{938}$ thin polymer films before $(0 \mathrm{~s})$ and after thermolysis at $240{ }^{\circ} \mathrm{C}$ for 260 and 540 seconds. Upon thermolysis the $v_{C=0}$ (ester, $1733 \mathrm{~cm}^{-1}$, dashed line) shifts to $1709 \mathrm{~cm}^{-1}$, which agrees with the absorbance of $v_{C=0}$ (carboxylic acid), followed by a shift to $1752 \mathrm{~cm}^{-1}$ and $1804 \mathrm{~cm}^{-1}$, which corresponds to absorbances of $v_{C=O}$ (anhydride). During thermolysis the absorbances corresponding to $\delta_{\mathrm{C}-\mathrm{H}}$ (tBA methyl group) between $1350 \mathrm{~cm}^{-1}$ and $1400 \mathrm{~cm}^{-1}$ disappear.

Static water contact angle measurements on $\mathrm{PS}_{644}-b-\mathrm{P} t \mathrm{BA}_{938}$ films as a function of thermolysis time at $250{ }^{\circ} \mathrm{C}$ are shown in Figure 7.4. The initial value of the water contact angle agrees well with the values reported in the literature for $\mathrm{P} t \mathrm{BA}$ surfaces. ${ }^{15}$ From X-ray photoelectron spectroscopy (XPS) surface analysis it is known that $\mathrm{P} t \mathrm{BA}$ forms an approximately $8 \mathrm{~nm}$ thick skin layer in these films (see also Chapter 6). ${ }^{15}$ Upon increasing thermolysis time from 0 to approximately 150 seconds, the values of the water contact angle decreased from $86^{\circ}$ to $66^{\circ}$. Prolonged thermolysis resulted in an increase of the water contact angle to $78^{\circ}$ for 300 seconds of thermolysis. Thermolysis for 1 hour yielded films with a water contact angle of $85^{\circ}$. The initial decrease in water contact angles for thermolysis times around approximately 150 seconds at $250{ }^{\circ} \mathrm{C}$ indicates that carboxylic acid groups are formed, which is in agreement with the proposed mechanism in Figure 7.1. We expect that these groups can be activated via e.g. EDC/NHS chemistry for functionalization with primary amine containing molecules. An explanation for the observed increase in water contact angle for longer thermolysis times is an enhanced polymer mobility at these thermolysis temperatures, which is high enough to facilitate the formation of a PS skin layer (see Chapter 6). ${ }^{24}$ 


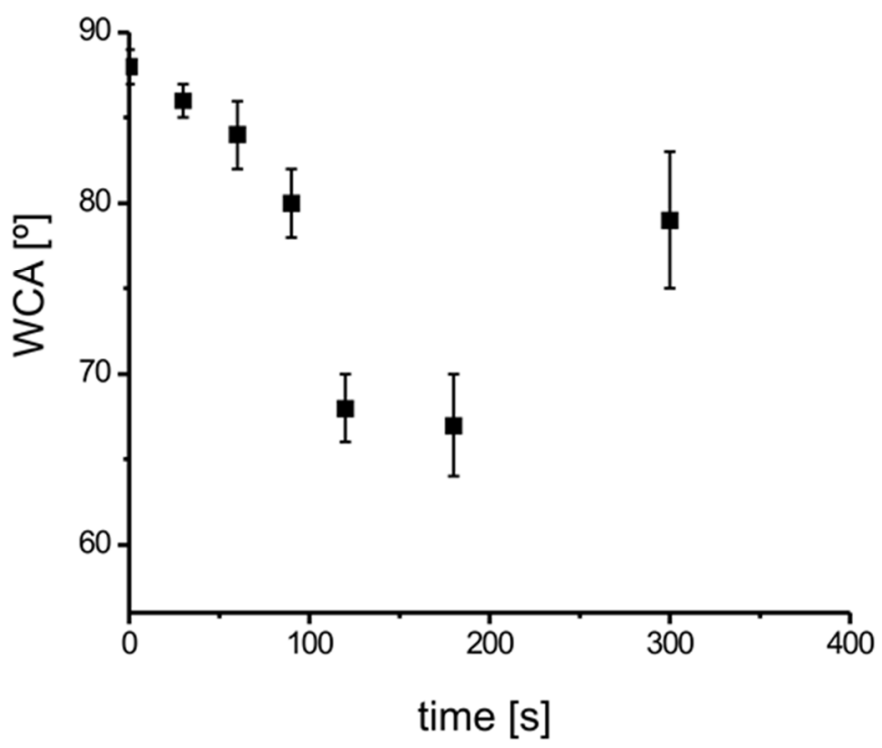

Figure 7.4: Static water contact angles of thermally activated $P S_{644}-b-P t B A_{938}$ thin films as a function of thermolysis time at $250{ }^{\circ} \mathrm{C}$. Following thermolysis, the films were incubated in a phosphate buffered saline solution (PBS, pH 7.4) for 12 hours to convert all anhydride functionalities into carboxylic acid moieties. The error bars represent the standard deviation $(n=6)$.

\subsubsection{Thermolysis kinetics}

From the FTIR spectra of thermolyzed $\mathrm{PS}_{644}-b-\mathrm{P} \mathrm{BA}_{938}$ films, the normalized fraction of $t \mathrm{BA}$ ester, carboxylic acid and anhydride groups was determined as a function of thermolysis time for reaction temperatures of $230^{\circ} \mathrm{C}, 240{ }^{\circ} \mathrm{C}$ and $250{ }^{\circ} \mathrm{C}$. The peak heights of characteristic absorbances of the corresponding functional groups were analyzed with respect to the absorbances attributed to poly(styrene) $\left(v_{s}(C-C)_{1455}\right.$ at $1455 \mathrm{~cm}^{-1}$ ), according to the following equations:

$$
\begin{aligned}
& \mathrm{f}_{\text {tert butyl ester }}=\left(\delta_{\mathrm{s}}(\mathrm{C}-\mathrm{H})_{1368} / \mathrm{v}_{\mathrm{s}}(\mathrm{C}-\mathrm{C})_{1455}\right)_{\mathrm{t}} /\left(\delta_{\mathrm{s}}(\mathrm{C}-\mathrm{H})_{1368} / \mathrm{v}_{\mathrm{s}}(\mathrm{C}-\mathrm{C})_{1455}\right)_{\mathrm{t} 0} \\
& \mathrm{f}_{\text {anhydride }}=0.6 \times\left(\mathrm{v}_{\mathrm{s}}(\mathrm{C}=\mathrm{O})_{1755} / \mathrm{v}_{\mathrm{s}}(\mathrm{C}-\mathrm{C})_{1455}\right)_{\mathrm{t}} /\left(\mathrm{v}_{\mathrm{s}}(\mathrm{C}=\mathrm{O})_{1755} / \mathrm{v}_{\mathrm{s}}(\mathrm{C}-\mathrm{C})_{1455}\right)_{\text {tmax }} \\
& \mathrm{f}_{\mathrm{COOH}}=1-\mathrm{f}_{\text {anhydride }}-\mathrm{f}_{\text {tert butyl ester }}
\end{aligned}
$$

The fractions of carboxylic acid were determined by assuming equation 3 , since it was impossible to quantify accurately the peak height of $v_{s}(C=O)_{1709}$ because of the relatively low carboxylic acid carbonyl absorbances and the overlapping of the remaining $t$ BA ester carbonyl absorbances. 
Results for the thermolysis at $250{ }^{\circ} \mathrm{C}$ are shown in Figure 7.5. The normalized $t$ BA ester fraction decreased within 120 seconds from 1.0 to 0 . Upon the disappearance of the $t \mathrm{BA}$ ester groups, the fractions of carboxylic acid groups and anhydride groups immediately increased. After $100 \%$ conversion of the $t$ BA ester to carboxylic acid groups, these groups were completely converted to anhydride groups. For longer thermolysis times the fraction of anhydride groups decreased. Most likely this is due to decarboxylation of the anhydride, which leads to the formation of carbon dioxide and ketons, as was reported by McNeill and Sadeghi. ${ }^{25}$ Although no direct evidence of the formed reaction products was found in the FTIR spectra, these authors reported that decarboxylation of PAA homopolymer occurs at temperatures above $210^{\circ} \mathrm{C}$. At temperatures of $250{ }^{\circ} \mathrm{C}$ and higher decarboxylation of the anhydride was significant. Later McGaugh and Kottle investigated the thermal degradation of poly(acrylic acid-co-ethylene) and found decarboxylation of the anhydride for thermolysis temperatures above $225{ }^{\circ} \mathrm{C}^{26}$ Significant decarboxylation might also explain the decrease in water contact angle as described above for longer thermolysis times. This process would lead to reduced functionality of the polymer film surfaces for too high thermolysis temperatures and too long thermolysis times.

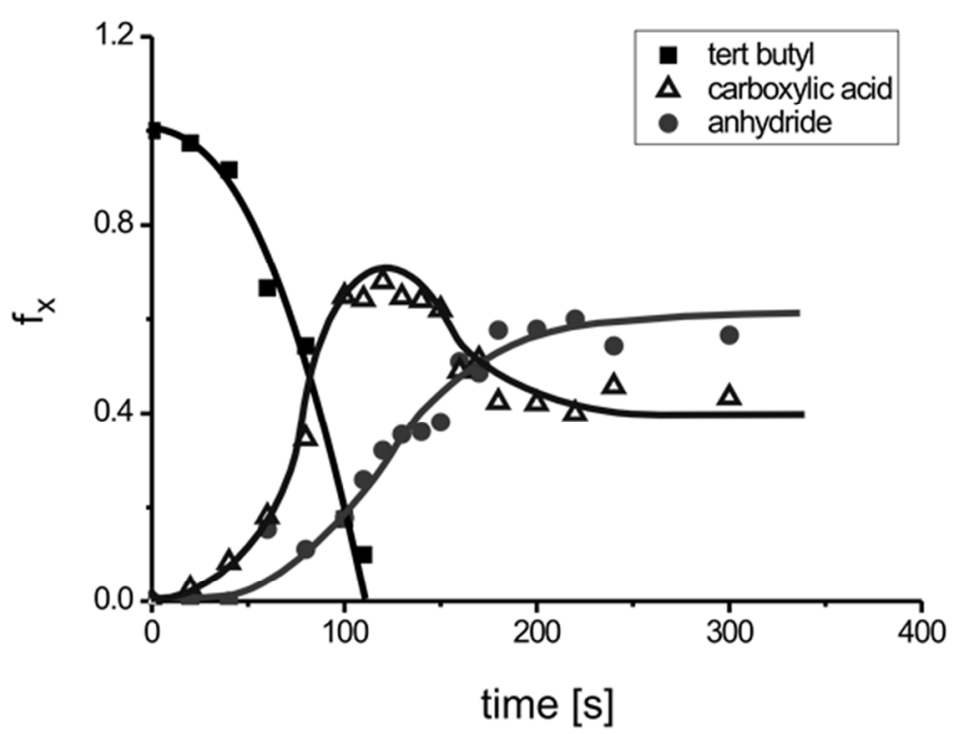

Figure 7.5: Normalized fractions of tBA ester, carboxylic acid and anhydride groups of $P S_{644}-b-P t B A_{938}$ films thermolyzed at $250{ }^{\circ} \mathrm{C}$ as a function of time. The solid lines are guides for the eye. The fraction of $\mathrm{BAA}$ ester and anhydride groups were determined from the FTIR spectra by analyzing the peak heights of the methyl and anhydride absorbances at $\delta_{\mathrm{C}-\mathrm{H}} 1368$ and $v_{C=0} 1804$, respectively, normalized to the peak heights of $v_{C-C}$ aromatic of $P S$ at $1453 \mathrm{~cm}^{-1}$ as a function of time. The fraction of carboxylic acid was calculated according to equation 3. 


\subsubsection{Apparent activation energy of $t \mathrm{BA}$ ester thermolysis}

Figure 7.6 shows the fraction of $t \mathrm{BA}$ ester of PS- $b-\mathrm{P} t \mathrm{BA}$ films as a function of thermolysis time for reactions carried out at temperatures of $230{ }^{\circ} \mathrm{C}, 240{ }^{\circ} \mathrm{C}$ and $250{ }^{\circ} \mathrm{C}$, respectively. Upon increasing the thermolysis temperature the time required to completely convert the $t \mathrm{BA}$ ester to carboxylic acid groups decreased. From this graph the half life of the reactions $\left(\tau_{1 / 2}\right)$ were determined to calculate the apparent activation energy of the $t \mathrm{BA}$ ester thermolysis.

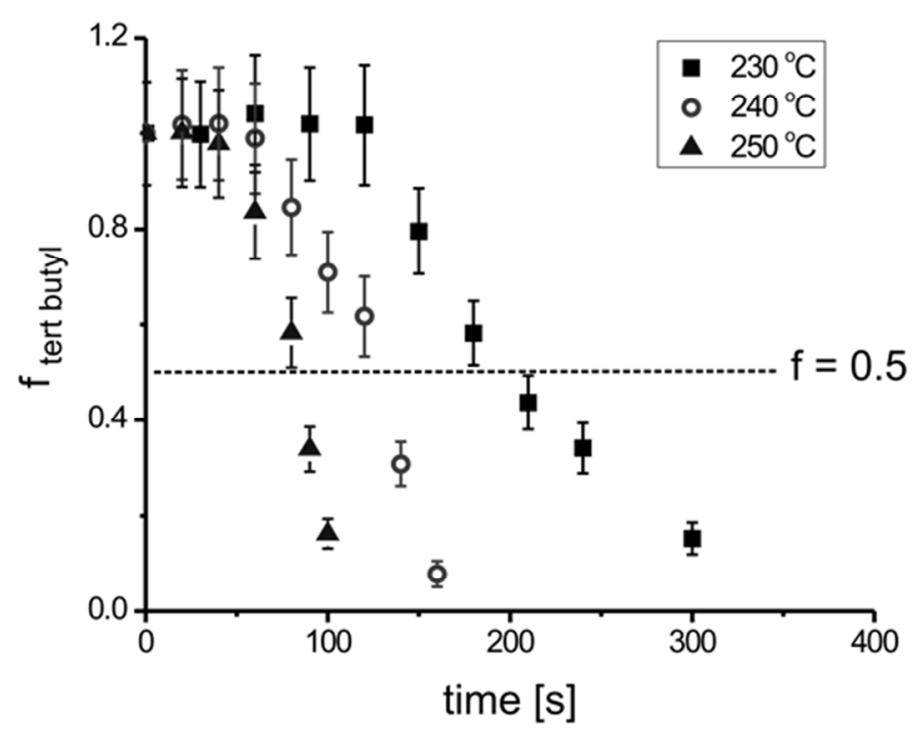

Figure 7.6: Normalized fractions of $t \mathrm{BA}$ ester groups of thermolyzed $P S_{644}-b-P t B A_{938}$ films as a function of time for different temperatures. From this plot $\tau_{1 / 2}$ of the $t B A$ ester thermolysis reaction at $230^{\circ} \mathrm{C}, 240{ }^{\circ} \mathrm{C}$ and $250^{\circ} \mathrm{C}$ was determined.

The apparent activation energy was calculated as:

$$
E_{a}=\frac{\ln \left(\frac{\tau_{1 / 2(1)}}{\tau_{1 / 2(2)}}\right)}{\frac{1}{R T_{1}}-\frac{1}{R T_{2}}}
$$

which was derived from Arrhenius equation by taking into account that $\tau_{1 / 2} \propto k^{-1}$ and by assuming, that the reaction order is constant throughout the course of the reaction. With the obtained values of $\tau_{1 / 2}$ and the corresponding thermolysis temperatures the apparent activation energy was calculated to be $93 \pm 12 \mathrm{~kJ} \mathrm{~mol}^{-1}$. This value is in good agreement with the value reported by Litmanovich and Cherkezyan for the autocatalytic part of the bulk thermolysis of P $t$ BA homopolymer. ${ }^{19}$ 


\subsubsection{Chemical activation and surface immobilization of fluoresceinamine}

The carboxylic acid groups exposed at the surface of thermolyzed $\mathrm{PS}_{644}-b-\mathrm{P} t \mathrm{BA}_{938}$ films were chemically activated with EDC/NHS and derivatized with a fluorescent dye to confirm their availability for surface reactions. Figure 7.7 shows the normalized green intensity count for samples that were thermally activated and subsequently modified with fluoresceinamine as a function of thermal activation time and thermal activation temperature. The maximum in this plot is observed at longer thermolysis times for lower thermolysis temperatures. Maximum surface functionalization was found at 140 seconds, 173 seconds and 338 seconds for thermolysis carried out at $250{ }^{\circ} \mathrm{C}, 240^{\circ} \mathrm{C}$ and $230{ }^{\circ} \mathrm{C}$, respectively. These times are in close proximity to the times required for $100 \%$ conversion of $\mathrm{P} t \mathrm{BA}$ to $\mathrm{PAA}$ as was determined by FTIR spectroscopy (Figure 7.6). These data are not identical, which can be explained by the fact that fluorescence microscopy probes the dye coupled to surface functional groups, while FTIR spectroscopy analyzes all functional groups throughout the entire film. The maximum in fluoresceinamine coverage for thermal activation at $250{ }^{\circ} \mathrm{C}$ corresponds well to the thermolysis time for which a minimum in water contact angle was observed (Figure 7.4).

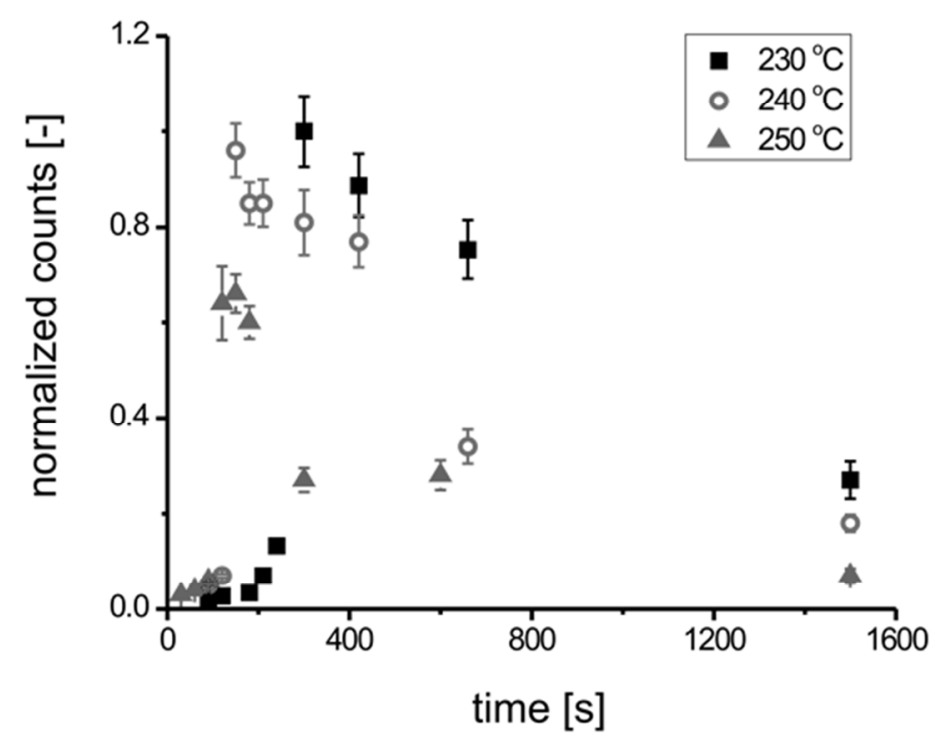

Figure 7.7: Normalized fluorescence microscopy intensity counts as a function of thermolysis time and thermolysis temperature of $P S_{644^{-}-b-P t B A_{938}}$ films covalently modified with fluoresceinamine following EDC/NHS activation.

Interestingly, for higher thermolysis temperatures, a lower maximum in green intensity counts was observed. This reduced fluoresceinamine coverage might be explained by the fact that decarboxylation of the anhydride occurs at temperatures above $200{ }^{\circ} \mathrm{C}$, as was reported by McNeill and Sadeghi. ${ }^{25}$ As a result fewer carboxylic acid groups are available for further surface modification. Another possibility for the 
lower surface functionality might be that the possible rearrangement of carboxylic acid groups away from the polymer film-air interfaces plays a bigger role at higher temperatures. Upon cooling down to room temperature these carboxylic acid groups might be frozen in the film being no longer available for subsequent surface immobilization of e.g. fluoresceinamine. These explanations are in agreement with the measured minimum in the water contact angle measurements, in which only the first few nanometers of the polymer film are probed.

Thus, from the combined results of TGA, FTIR spectroscopy, contact angle measurements and fluorescence microscopy the time and temperature windows were determined, in which maximum conversion of PtBA to PAA and PAnH, as well as maximum surface functionalization are obtained. Thermolysis temperatures above $230^{\circ} \mathrm{C}$ result in complete conversion of the PtBA block to PAA and PAnH, while at temperatures around $250{ }^{\circ} \mathrm{C}$ the degree of surface functionalization drops dramatically due to decarboxylation.

\subsubsection{Local thermal surface functionalization}

For various applications localized surface functionalization is required, as outlined in the introduction. To establish the concept of local thermal surface activation by scanning probe methods and subsequent functionalization, $\mathrm{PS}_{2092}$-b-P $t$ BA 1055 films were initially thermolyzed using a heated copper constantan wire (diameter $1 \mathrm{~mm}$ ). The wire was brought into close contact with the film surface and subsequent chemical activation and covalent immobilization of $\alpha$-amines was employed to test the viability of this approach for thermal surface patterning.

In the experiments controlled Joule heating of the wire bent in different geometries was used to locally thermally activate the films. The subsequent covalent functionalization in the thermally activated areas with $\mathrm{MeO}-\mathrm{PEG}_{45}-\mathrm{NH}_{2}$ was employed to render these area passivated against non-specific adsorption of proteins. ${ }^{27}$ The passivating properties of the thermally activated areas was shown in experiments with fluorescently labeled bovine serum albumin (BSA). Figure 7.8A shows that the PEG layer successfully prevents non-specific protein adsorption. Complementary to these data are fluorescence microscopy images for samples that were functionalized with fluoresceinamine in the deprotected areas, as shown in Figure 7.8B. Local thermal functionalized areas can be functionalized with similar functionalities as was reported by us earlier for chemically hydrolyzed and activated PS- $b$-P $t$ BA block copolymer films. ${ }^{15}$ Control experiments in which the EDC/NHS activation of the carboxylic acid groups was omitted confirmed that the fluorescence emission in the images shown in Figure 7.8 is not a result of local physical adsorption of PEG or fluoresceinamine. Nonchemical surface alternation effects in the locally thermally 
deprotected areas were also excluded as possible interpretation (insets in Figure 7.8). Depending on the wire geometry the smallest width of thermally activated area obtained was around $120 \mu \mathrm{m}$. Irregularities in the fluorescence microscopy images are explained by the fact that the wire was not brought into contact with the polymer film in a controlled way. As a result the wire may have moved upon heating, thereby scratching the polymer film. However, the complementarity of the labeling approaches demonstrates that local thermolysis is a feasible approach for generating small chemical patterns at surfaces.

A

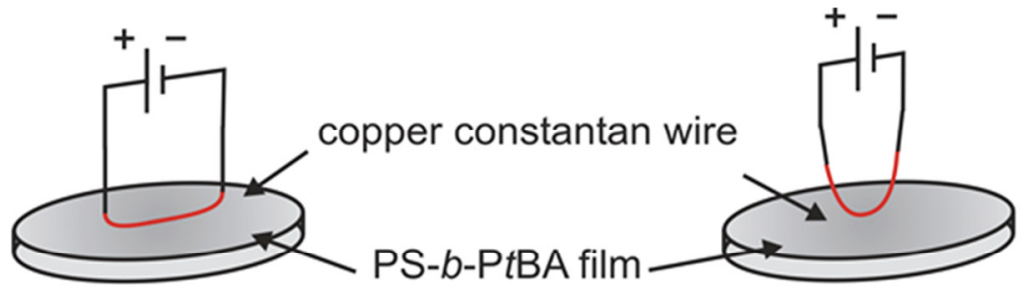

B
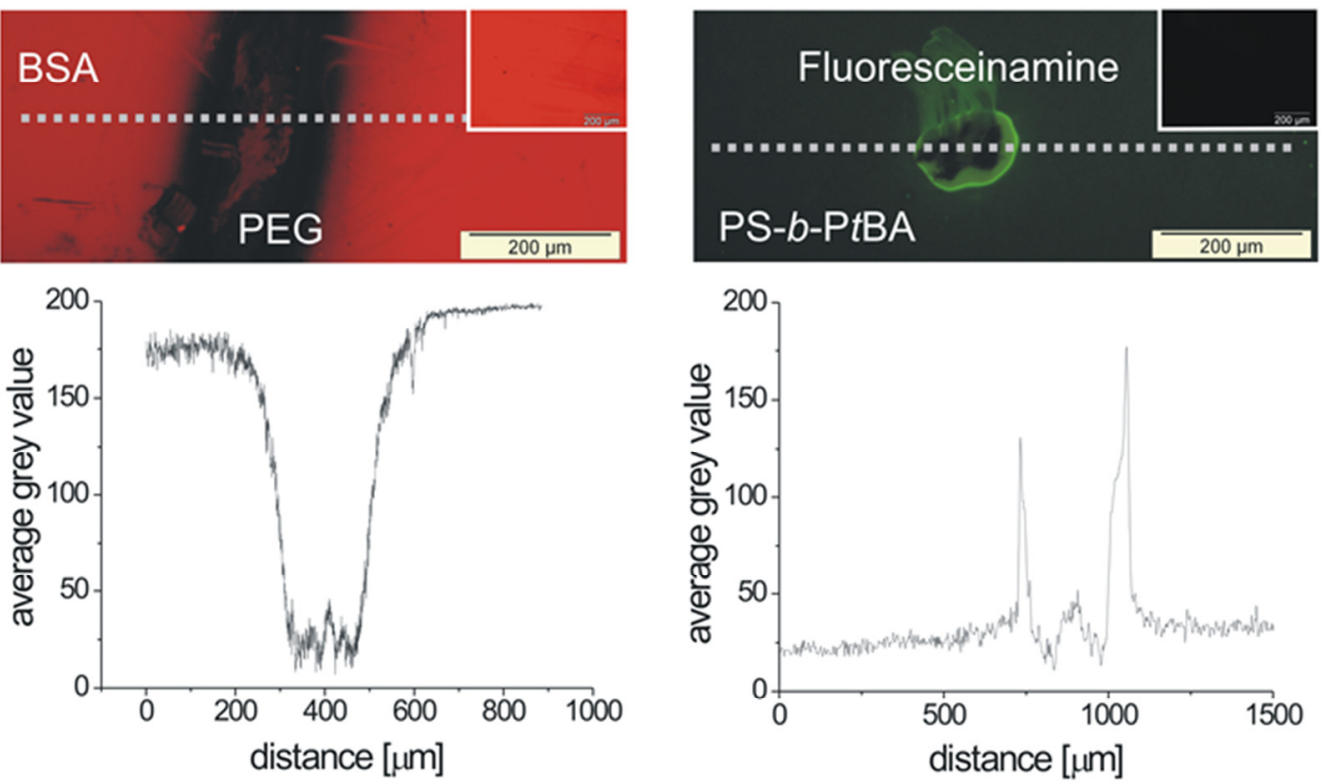

Figure 7.8: Fluorescence microscopy images of locally thermally deprotected $P S_{2092}-b-P t B A_{1055}$ thin film areas following chemical activation and derivatization. $(A)$ : The thermally activated areas were passivated with a covalently coupled $\mathrm{MeO}-\mathrm{PEG}{ }_{45}-\mathrm{NH}_{2}$ layer to prevent subsequent non-specific adsorption of dye-labeled BSA. In this case the wire was parallel to the surface. (B): After thermal activation fluoresceinamine was covalently immobilized. The wire was bended to a point to provide a small contact area. The inset in the fluorescence microscopy images show results for control experiments in which no EDC/NHS activation of the locally thermally deprotected areas was applied, the scale bar reads $200 \mu \mathrm{m}$.

Based on the promising results from this simple millimeter to micrometer heatable wire experiments the length scales of thermal surface activation were pushed down to sub- $\mu \mathrm{m}$ length scales by using heatable atomic force microscopy probes. In the first approach a heated probe $\left(T_{i}=265^{\circ} \mathrm{C}\right)$ was brought into contact with the $\mathrm{PS}_{644}-b-\mathrm{P} t \mathrm{BA}_{938}$ film in order to raster scan a $30 \mu \mathrm{m} \times 30 \mu \mathrm{m}$ square (Figure $7.9 \mathrm{~A}$ ). 
A
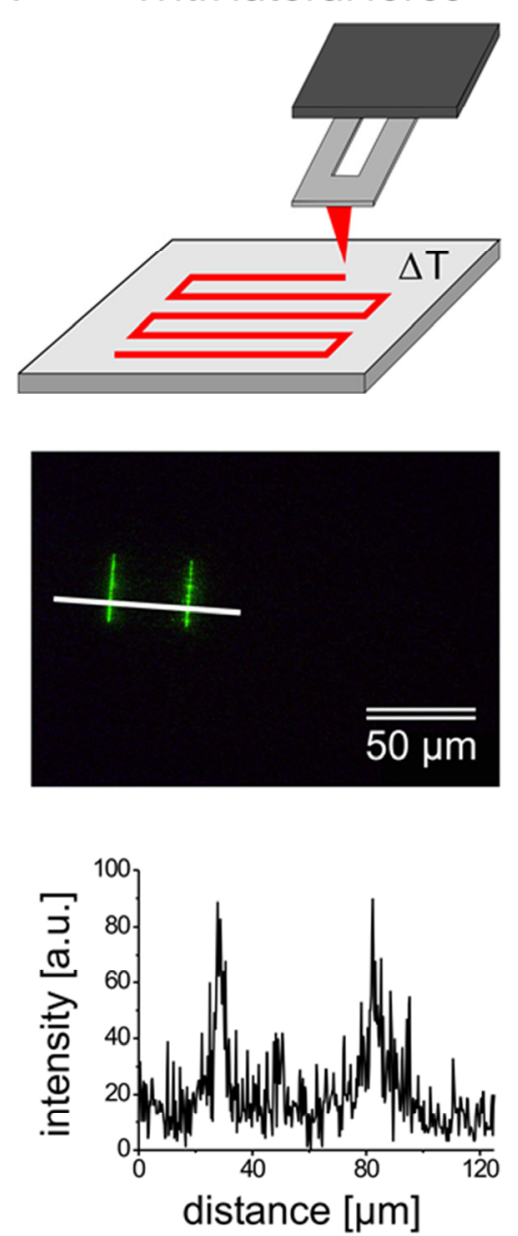

B No lateral force
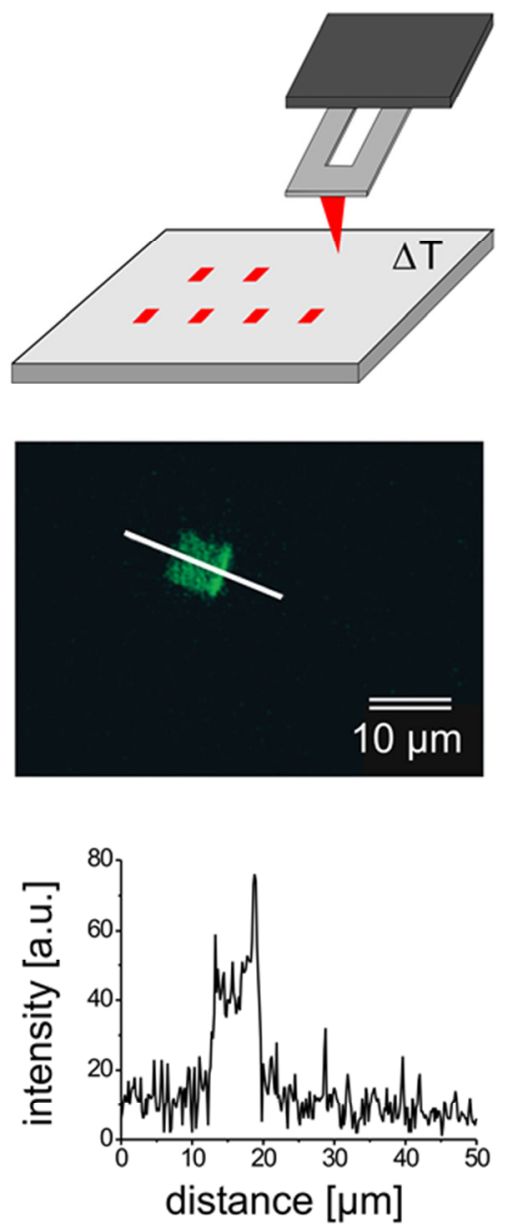

Figure 7.9: Schematic of scanning thermal microscopy approaches (top) and fluorescence microscopy images (middle) and cross-sections (bottom) of squares on PS 644- $_{-b-P t B A_{938}}$ films prepared by raster scanning in contact mode (A) a $30 \mu \mathrm{m} \times 30 \mu \mathrm{m}$ square (tip velocity $\left.\sim 30 \mu \mathrm{m} \mathrm{s}^{-1}\right)$ and $(B)$ by indenting an array of 25 points $\times 25$ points $(x-y$ separation $250 \mathrm{~nm}$, contact time $1 \mathrm{~s}$ per point) with a heated probe $\left(T=265^{\circ} \mathrm{C}\right)$. The applied contact loads for SThL were $\sim 10 \mathrm{nN}$. Following EDC/NHS activation, fluoresceinamine was covalently immobilized in these areas. For illustrative purpose the fluorescence microscopy images were filtered. The cross-sections shown are the original cross-sections.

After covalent immobilization of fluoresceinamine it turned out that most of the thermolyzed material was moved to the sides perpendicular to the probe scanning direction presumably due to the high lateral forces. This effect was confirmed by contact mode AFM analyses of the thermally functionalized areas after thermal activation with SThL (Figure 7.10A). Elevated ridges of approximately $100 \mathrm{~nm}$ in height were observed. In order to reduce the high lateral forces, an array of 25 points by 25 points was indented with a heated probe (probe tip temperature $\left(T_{i}\right)=265^{\circ} \mathrm{C}$ ). This second approaches resulted in a more homogeneous thermally activated film (Figure 7.9B). The indentations left behind were $\sim 370 \mathrm{~nm} \times 580 \mathrm{~nm}$ wide and $30 \mathrm{~nm}$ deep (Figure 7.10B). 
A

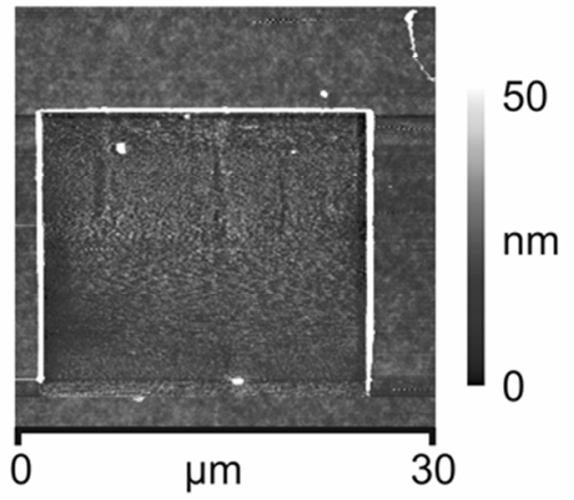

C

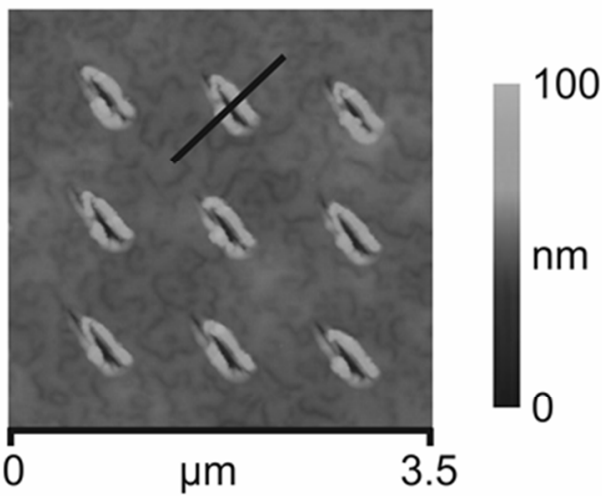

B

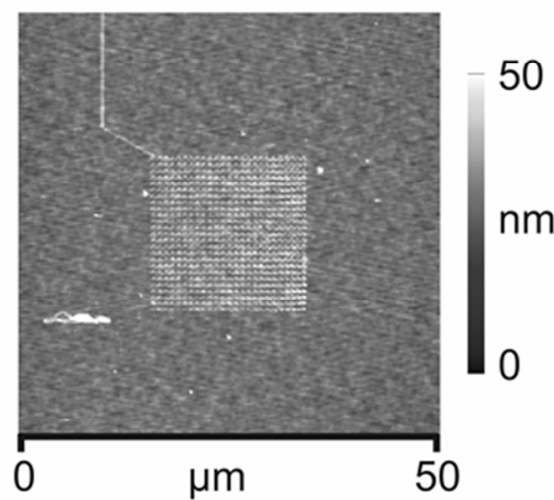

D

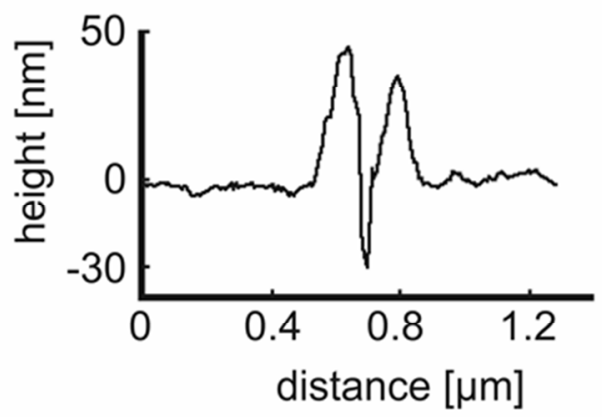

Figure 7.10: $A F M$ height images of $P S_{644}-b-P t B A_{938}$ areas thermally activated via SThL $\left(T_{i}=265{ }^{\circ} \mathrm{C}\right.$, contact load $\sim 10 \mathrm{nN}$ ) according to the two approaches described in Figure 7.9. (A) AFM height image shows a raster scanned square of $20 \mu \mathrm{m}$ by $20 \mu \mathrm{m}$ (tip velocity $\sim 20 \mu \mathrm{m} \mathrm{s}^{-1}$ ); (B) AFM height image shows an array of indents with a $\mathrm{x}$ and $\mathrm{y}$ spacing of $750 \mathrm{~nm}$ (contact time $1 \mathrm{~s}$ per point). (C) AFM height image of some indents produced as in (B) with $\mathrm{x}$ and $\mathrm{y}$ spacing $1 \mu \mathrm{m}$; (D) cross-section along the black line of an indent shown in $(C)$.

Since each of the 525 depressions measures only $370 \mathrm{~nm} \times 580 \mathrm{~nm}$ in width, these features cannot be resolved in the fluorescence microscopy images. The clear fluorescence emission (square shape) detected indicates that the covalent modification with the fluorescent dye was very efficient. The resolution limits of this new SPL approach are not established yet, but temperature, duration of the tip-sample contact, and feedback settings, among others, are expected to contribute to attainable minimum feature size and separation.

In Chapter 4 the proximity of silicon substrates in proximity of the heated probe tip-sample contact interface was described in terms of a deviation in $T_{i}$ from the calibrated $T_{i}\left(\Delta T_{i}\right)$. The mentioned $T_{i}$ of $265{ }^{\circ} \mathrm{C}$ for the patterns produced as shown in Figure 7.9 and Figure 7.10 is the uncorrected value. Based on the results provided in Chapter 4 and the thickness of the polymer films reported here $(\sim 98 \mathrm{~nm})$ a correction 
for $T_{i}$ of $\sim 32{ }^{\circ} \mathrm{C}$ is suggested. Hence, $T_{i}$ used during $\mathrm{SThL}$ as described in this chapter was approximately $233{ }^{\circ} \mathrm{C}$.

Having established all needs for the spatially controlled thermal chemical surface functionalization, the next chapter will focus on the development of polymer film platforms with improved thermomechanical stability for sub 50 nanometer length scale thermochemical surface functionalization by SThL.

\subsection{Conclusions}

The concept of local thermal activation of thin polymer films for area-selective surface chemical modification on micrometer and nanometer length scales with SThL was introduced. The kinetics and mechanism of the thermally induced activation of $t$ BA ester moieties in PS- $b$-P $t$ BA block copolymer films was quantitatively analyzed and the apparent activation energy $\left(E_{a}\right)$ for the $t \mathrm{BA}$ ester deprotection in thin films was calculated to be $93 \pm 12 \mathrm{~kJ} \mathrm{~mol}^{-1}$. The carboxylic acid groups generated locally using a heatable wire and SThL, down to length scales of $370 \mathrm{~nm} \times 580 \mathrm{~nm}$, were successfully chemically activated and covalently functionalized with fluoresceinamine and PEG. These results are very promising as they demonstrate the successful application of SThL in conjunction with a tailored block copolymer platform with established bioconjugation strategies that is stable under conditions for wet chemical processing. Thus, truly tailored patterned biointerfaces for cell-surface interaction studies can in principle be realized with SThL.

\subsection{Experimental}

Materials: $\mathrm{PS}_{\mathrm{n}}-b-\mathrm{P} t \mathrm{BA}_{\mathrm{m}}$ (with degrees of polymerization $\mathrm{n}, \mathrm{m}=644,938$ and 2092 , 1055) were purchased from Polymer Source Inc. (Dorval, Canada). The total molar mass of these polymers is $187 \mathrm{~kg} \mathrm{~mol}^{-1}$ with a polydispersity of 1.05 and $352 \mathrm{~kg} \mathrm{~mol}^{-1}$ with a polydispersity of 1.10 , respectively. Amino end-functionalized poly(ethylene glycol) $\left(\mathrm{MeO}-\mathrm{PEG}_{45}-\mathrm{NH}_{2}, \mathrm{MW}=2 \mathrm{~kg} \mathrm{~mol}^{-1}\right)$ was obtained from Iris Biotech $\mathrm{GmbH}$ (Marktredwitz, Germany), phosphate buffered saline solution (PBS, pH 7.4) from B. Braun (Melsungen, Germany). Toluene and ethanol were purchased from Biosolve (Valkenswaard, the Netherlands). Fluoresceinamine isomer I ( $\left.\lambda_{\max }=496 \mathrm{~nm}\right)$, 1-ethyl3-(3-dimethylaminopropyl)carbodiimide hydrochloride (EDC) and N-hydroxysuccinimide (NHS) were bought from Sigma-Aldrich (St. Louis, MO, USA). Bovine Serum Albumin Alexa Fluor ${ }^{8} 54$ conjugate was obtained from Molecular Probes/ Invitrogen Co. (Carlsbad, CA, USA). All compounds were used without further 
purification. Milli-Q water was produced by a Millipore Synergy system (Billireca, MA, USA).

Preparation of thin films: Block copolymer thin films were prepared by spin-coating polymer solutions (typically $2 \mathrm{wt} \%$ of polymer in toluene, filtered with a $0.2 \mu \mathrm{m}$ Whatman Spartan 13 filter) onto pre-cleaned silicon substrates (CZ, type P, Boron, $<100>$, diameter $100 \mathrm{~mm}$, OKMETIC, Vantaa, Finland) at $3.000 \mathrm{rpm}$ for 20 seconds (Suss MicroTec Delta20 spincoater, Garching/Hochbrück, Germany). Prior to usage the silicon substrates were cleaned in a $\mathrm{HNO}_{3}$ bath for 10 minutes, extensively rinsed with Milli Q water, treated in another $\mathrm{HNO}_{3}$ bath for 10 minutes (boiling), extensively rinsed with Milli Q water and finally spin dried. Spin cast films were either annealed at $130{ }^{\circ} \mathrm{C}$ in vacuum for 12 hours or were vacuum dried at room temperature for 12 hours. Mean film thicknesses were determined using a Plasmos SD 2002 ellipsometer (Munich, Germany) at a fixed wavelength and angle of $632.8 \mathrm{~nm}$ and $70^{\circ}$, respectively. A refractive index of the block copolymer films of 1.513 was used in the calculations. ${ }^{15}$ The mean film thickness measured was $98 \pm 2.1 \mathrm{~nm}$. For subsequent experiments $1 \mathrm{~cm} \times 1 \mathrm{~cm}$ sized samples were cut from the polymer filmcovered wafers.

Thermo gravimetric analysis: The isothermal weight loss of $\mathrm{PS}_{644}-b-\mathrm{P} t \mathrm{BA}_{938}$ polymer samples $(10 \mathrm{mg})$ under nitrogen was measured as a function of time at different temperatures with a Perkin Elmer Thermo Gravimetric Analyzer (TGA 7, Waltham, MA, USA). The initial temperature ramp from room temperature to thermolysis temperature was set to $100{ }^{\circ} \mathrm{C} \mathrm{min}^{-1}$ using a constant nitrogen flow of $20 \mathrm{ml} \mathrm{min}^{-1}$.

Thermal activation of polymer thin films: The thermolysis of thin films was carried out at temperatures of $230{ }^{\circ} \mathrm{C}, 240{ }^{\circ} \mathrm{C}$ or $250{ }^{\circ} \mathrm{C}$ in a small flask $(25 \mathrm{ml})$ positioned in a thermostatted oil bath (silicon oil AP100, Sigma Aldrich, St Louis, USA). Prior to sample loading, the flask was purged with nitrogen for approximately 5 minutes. The temperature inside and outside the flask was monitored. The films were placed inside the flask and the flask was sealed with a glass stopper. After the desired thermolysis time samples were taken out and quickly cooled to room temperature under a nitrogen stream.

Fourier transform infrared spectroscopy: Transmission mode FTIR spectra (spectral resolution $4 \mathrm{~cm}^{-1}$, 1024 scans) were collected with a BIO-RAD model FTS575C FTIR spectrometer equipped with a liquid nitrogen-cooled cryogenic mercury cadmium telluride detector (Hercules, CA, USA). The background spectra were obtained on piranha cleaned silicon substrates.

Coupling of MeO-PEG-NH $\mathrm{H}_{2}$ or fluoresceinamine: Freshly thermally activated polymer films were incubated in Milli-Q water for 1 hour followed by activation of the carboxylic acid groups via EDC/NHS chemistry $(200 \mathrm{mM}$ and $250 \mathrm{mM}$, 
respectively, in PBS). After EDC/NHS activation for 1 hour the samples were rinsed with PBS 3 times and immersed for 1 hour in either a $\mathrm{MeO}-\mathrm{PEG}_{45}-\mathrm{NH}_{2}$ or fluoresceinamine solution in PBS (concentration $1.0 \times 10^{-4} \mathrm{M}$ ). Fluoresceinamine modified films were extensively rinsed with Milli-Q water before drying in a nitrogen stream. PEG-ylated samples were rinsed 3 times with PBS and placed in a BSA Alexa Fluor ${ }^{\circledR}$ conjugate solution (concentration $1.0 \times 10^{-5} \mathrm{M}$ ) in PBS for 1 hour to fluorescently label the non-PEG-ylated film areas via non-specific protein adsorption of BSA. Subsequently, the films were extensively rinsed with Milli-Q water and dried in a nitrogen stream.

Fluorescence microscopy: Fluorescence microscopy images of the dry polymer films were taken directly after preparation of the samples with an Olympus IX71 fluorescence microscope. For the excitation of BSA labeled with Alexa Fluor ${ }^{\circledR} 594$ an U-MWB-2 filter cube combined with a DM570 dichroic mirror and BA590 barrier filter was used, while for the excitation of fluoresceinamine only a U-MWB-2 filter cube was used. Typical sample exposure times were $50 \mathrm{~ms}$. Fluorescence microscopy images were analyzed with Image J (National Institutes of Health, version 1.38, freeware).

Contact angle measurements: Static water contact angles (CA) of thermally activated films were measured with Milli-Q water as a probe liquid using a CA microscope (Data Physics OCA model 15 Plus, Filderstadt, Germany) at room temperature and ambient humidity. Freshly prepared thermolyzed samples were incubated in Milli Q water for 1 hour and dried under a nitrogen stream prior to the water contact angle measurements. Data for 3 different spots on each sample were averaged.

Heatable wire setup: Using a Delta Elektronika SM7020-D power supply (Zierikzee, the Netherlands) $2.6 \mathrm{~V}$ and $5 \mathrm{~A}$ were applied to a $1 \mathrm{~mm}$ diameter copper constantan wire to reach a calibrated temperature $\left(250{ }^{\circ} \mathrm{C}\right)$. Calibration was done by bringing the wire in contact with a thermocouple (5TC-TT-T-36-36, Omega Engineering Inc., Stamford, CT, USA ). Good thermal contact was provided by adding some thermal conductive paste (thermal compound part no. 120-2, Wakefield Engineering Inc., Pelham, NH, USA). The estimated error in temperature is $\pm 5{ }^{\circ} \mathrm{C}$. Local contact heating of $\mathrm{PS}_{2092}-b-\mathrm{P} t \mathrm{BA}_{1055}$ polymer films was carried out using different wire geometries and different heating times.

Scanning thermal lithography: Heatable AFM probes (type AN-2, with $200 \mu \mathrm{m}$ long cantilevers, Anasys Instruments, Santa Barbara, CA, USA) mounted in an Asylum research MFP-3D AFM (Santa Barbara, CA, USA) were used for SThL. The probe temperature was set to $265{ }^{\circ} \mathrm{C}$ with a Nano-TA2 controller (Anasys Instruments) using the following standard polymer samples: ${ }^{28}$ poly( $\varepsilon$-caprolactone) (PCL, $T_{m} \sim 55^{\circ} \mathrm{C}$ ), poly(ethylene) (PE, $T_{m} \sim 116{ }^{\circ} \mathrm{C}$ ) and poly(ethylene terephthalate) (PET, 
$T_{m} \sim 235^{\circ} \mathrm{C}$ ). After local thermal functionalization of the polymer films contact mode AFM images were taken with the same AFM probe. AFM analysis was also carried out in tapping mode with a Dimension D3100 equipped with a hybrid 153 scanner and a NanoScope IVa controller (Veeco/Digital Instruments (DI), Santa Barbara, CA) using silicon cantilevers (Nanosensors, Wetzlar, Germany) in ambient conditions.

\subsection{References and notes}

1 a) Falconnet, D.; Csucs, G.; Grandin, M. H.; Textor, M. Biomaterials 2006, 27, 3044. b) Truskett, V. N.; Watts, M. P. C. Trends Biotechn. 2006, 24, 312. c) Bernard, A.; Renault, J. P.; Michel, B.; Bosshard, H. R.; Delamarche, E. Adv. Mater. 2000, 12, 1067. d) Ge, Y.; Turner, A. P. F. Trends Biotechn. 2008, 26, 218.

2 Vettiger, P.; Despont, M.; Drechsler, U.; Dürig, U.; Häberle, W.; Lutwyche, M. I.; Rothuizen, H. E.; Stutz, R.; Widmer, R.; Binnig, G. K. IBM J. Res. Dev. 2000, 44, 323.

3 a) Böttger, G.; Liguda, C.; Schmidt, M.; Eich, M. App. Phys. Lett. 2002, 81, 2517. b) Wülbern, J. H.; Schmidt, M.; Eich, M.; Hübner, U.; Boucher, R.; Marlow, F.; Volksen, W. App. Phys. Lett. 2007, 91, 221104.

4 a) Mizutani, A.; Kikuchi, A.; Yamato, M.; Kanazawa, H.; Okano, T. Biomaterials 2008, 29, 2073. b) Lussi, J. W.; Michel, R.; Reviakine, I.; Falconnet, D.; Goessl, A.; Csucs, G.; Hubbell, J. A.; Textor, M. Progr. Surf. Sci. 2004, 76, 55. c) Chen, C. S.; Jiang, X.; Whitesides, G. M. MRS Bull. 2005, 30, 194.

5 a) Knoll, W.; Yu, F.; Neumann, T.; Schiller, S.; Naumann, R. Phys. Chem. Chem. Phys. 2003, 5, 5169. b) MacBeath, G.; Schreiber, S. L. Science 2000, 289, 1760. c) Wells, M.; Crooks, R. M. J. Am. Chem. Soc. 1996, 118, 3988.

6 Chou, S. Y.; Krauss, P. R. Microelectr. Eng. 1997, 35, 237-240.

7 a) Böker, A.; Reihs, K.; Wang, J.; Stadler, R.; Ober, C. K. Macromolecules 2000, 33, 1310. b) Böker, A.; Herweg, T.; Reihs, K. Macromolecules 2002, 35, 4929.

8 a) La, Y. H.; Edwards, E. W.; Park, S. M.; Nealey, P. F. Nano Lett. 2005, 5, 1379. b) La, Y. H.; Stoykovich, M. P.; Park, S. M.; Nealey, P. F. Chem. Mater. 2007, 19, 4538.

9 Szoszkiewicz, R.; Okada, T.; Jones, S. C.; Li, T. D.; King, W. P.; Marder, S. R.; Riedo, E. Nano Lett. 2007, 7, 1064.

10 Lee, J.; Beechem, T.; Wright, T. L.; Nelson, B. A.; Graham, S.; King, W. P. J. Microel. Syst. 2006, $15,1644$.

11 Piner, R. D.; Zhu, J.; Xu, F.; Hong, S. H.; Mirkin, C. A. Science 1999, 283, 661.

12 Sun, S.; Leggett, G. J. Nano Lett. 2004, 4, 1381.

13 Krämer, S.; Fuierer, R. R.; Gorman, C. B. Chem. Rev. 2003, 103, 4367.

14 Alvarez, L.; Xiao, M. J. Microscopy 2008, 229, 371.

15 a) Feng, C. L.; Vancso, G. J.; Schönherr, H. Langmuir 2005, 21, 2356. b) Feng, C. L.; Embrechts, A.; Vancso, G. J.; Schönherr, H. Eur. Polym. J. 2006, 42, 1954. c) Feng, C. L.; Embrechts, A.; Bredebusch, I.; Bouma, A.; Schnekenburger, J.; García-Parajó, M.; Domschke, W.; Vancso, G. J.; Schönherr, H. Eur. Polym. J. 2007, 43, 2177. d) Feng, C. L.; Vancso, G. J.; Schönherr, H. Langmuir 2007, 23, 1131.

16 Feng, C. L.; Embrechts, A.; Bredebusch, I.; Schnekenburger, J.; Domschke, W.; Vancso, G. J.; Schönherr, H. Adv. Mater. 2007, 19, 286. 
17 Schönherr, H.; Feng, C. L.; Tomczak, N.; Vancso, G. J. Macromol. Symp. 2005, 230, 149.

18 Pan, F.; Wang, P.; Lee, K.; Wu, A.; Turro, N. J.; Koberstein, J. T. Langmuir 2005, 21, 3605.

19 Litmanovich, A. D.; Cherkezyan, V. O. Eur. Polym. J. 1984, 20, 1041.

20 a) Alfrey, T.; Haas, H. C.; Lewis, C. W. J. Am. Chem. Soc. 1951, 73, 2851. b) Odian, G. Principles of Polymerization, $3^{\text {rd }}$ edition; John Wiley \& Sons Inc.: New York, 1991, p.692.

21 Schaefgen, J. R.; Sarasohn, I. M. J. Polym. Sci. 1962, 58, 1049.

22 Cherkezyan, V. O.; Litmanovich, A. D. Eur. Polym. J. 1985, 21, 623.

23 Litmanovich, A. D.; Cherkezyan, V. O. Polym. Sci. USSR 1985, 27, 2096.

24 Duvigneau, J.; Cornelissen, S.; Bardají Valls, N.; Schönherr, H.; Vancso, G. J. Adv. Funct. Mater. 2010, 20, 460.

25 McNeill, I. C.; Sadeghi, S. M. T. Polym. Degr. Stab. 1990, 29, 233.

26 McGaugh, M.C.; Kottle, S. J. Polym. Sci. Part A-1 1968, 6, 1243.

27 Jo, S.; Park, K. Biomaterials 2000, 21, 605.

28 In the calibration of the probe temperature using this procedure the softening of the polymer substrates is determined. Since polymers do not possess a priori a defined melting point, this procedure introduced some errors, which, as we note explicitly, are not relevant for the work discussed here. Recent progress in calibration of the probe temperature has been reported by Abel et al.: Abel, M. R.; Wright, T. L.; King, W. P.; Graham, S. IEEE Trans. Comp. Pack. Techn. 2007, 30, 200. See also Chapter 2. 



\section{Chapter 8}

\section{Tailored tert-Butyl Ester Protected Carboxylic Acid Functionalized (Meth)acrylate Polymer Platforms for SThL}

In this chapter the development of crosslinked polymer films bearing surface exposed tert-butyl ester protected carboxylic acid groups for sub 100 nanometer scanning thermal lithography (SThL) is described. Crosslinked polymer films comprising tert-butyl methacrylate $\left(\mathrm{MA}_{20}\right)$ or tert-butyl acrylate $\left(\mathrm{A}_{20}\right)$ were prepared via $\mathrm{UV}$ initiated free radical polymerization. Thermogravimetric analysis (TGA) and FTIR spectroscopy showed that upon heating $\mathrm{MA}_{20}$ thermal decomposition is initially the primary reaction mechanism followed by tert-butyl ester thermolysis. The values of the apparent activation energies $\left(\mathrm{E}_{\mathrm{a}}\right)$ for tert-butyl ester decomposition were calculated to be $125 \pm 13 \mathrm{~kJ} \mathrm{~mol}^{-1}$ and $116 \pm 7 \mathrm{~kJ} \mathrm{~mol}^{-1}$ for $\mathrm{MA}_{20}$ and $\mathrm{A}_{20}$, respectively. For both $\mathrm{MA}_{20}$ and $\mathrm{A}_{20}$ thermal decomposition of the tert-butyl ester results in the loss of isobutylene yielding carboxylic acid groups, followed by the formation of intramolecular anhydride groups through the loss of water. Both $\mathrm{MA}_{20}$ and $\mathrm{A}_{20}$ films showed improved thermomechanical stability during SThL compared to non crosslinked polymer films. During SThL no surface deformations other than those related to thermo chemical decomposition reactions were observed. The availability of carboxylic acid moieties in subsequent wet chemical grafting reactions with either fluoresceinamine or amine functionalized eFluor 605 quantum dots following well established EDC/NHS chemistry was confirmed with (confocal) fluorescence microscopy. The apparent activation energy for groove formation in $\mathrm{MA}_{20}$ films with SThL was calculated to be $45 \pm 3 \mathrm{~kJ} \mathrm{~mol}^{-1}$. Lines prepared by SThL in $A_{20}$ films had a typically $\sim 10$ times less width compared to lines written in $\mathrm{MA}_{20}$ films regardless of the tip radius of the heated probe used for SThL. This observation is ascribed to oxidative thermal depolymerization during $\mathrm{SThL}$ of $\mathrm{MA}_{20}$ films which is considered as the dominant reaction mechanism. The smallest line width values obtained for $\mathrm{MA}_{20}$ and $\mathrm{A}_{20}$ films with SThL were $83 \pm 7 \mathrm{~nm}$ and $21 \pm 2 \mathrm{~nm}$, respectively. 


\subsection{Introduction}

Scanning probe lithography (SPL) is among the most versatile tools to fabricate, manipulate and address surface domains with distinguished chemical and/or topological properties at the critical sub 100 nanometer length scale. ${ }^{1}$ Surface exposed domains that contain a specific type of information or functionality at these length scales are of great interest in different areas, e.g. cell-surface interaction studies, ${ }^{2}$ development of (bio)sensors, ${ }^{3}$ and data storage applications. ${ }^{4}$ Compared to other direct writing nanofabrication techniques (i.e. directed beam lithographies), ${ }^{5} \mathrm{SPL}$ is relatively cost effective and easy to operate in a broad range of environments on a wide variety of samples, including organic and biological materials. As potential alternatives, other recently established nano lithography approaches include soft lithography ${ }^{6}$ and nano imprint lithography (NIL). ${ }^{7}$ The various techniques of soft lithography mainly focus on the fabrication of chemical functional surface patterns in the sub micrometer range via printing with patterned elastomer stamps. NIL utilizes specially designed topographical nano patterned molds to imprint polymer films at elevated temperatures with sub 50 nanometer resolution. During imprinting at elevated temperatures thermal decomposition or other thermally induced reactions may take place, which are often undesired and potentially result in distorted pattern formation. In Chapter 7 the few known examples in which heat was exploited for the controlled chemical surface functionalization were identified. ${ }^{8}$ In Chapter 6 we have introduced the combined thermal chemical surface functionalization and topographic patterning of poly(styrene)-block-poly(tert-butyl acrylate) (PS- $b$-P $t$ BA) films for the development of topographically shaped chemically functional bio reactive interfaces. This technique is referred to as reactive imprint lithography (RIL) ${ }^{9}$ and comprises the imprinting of PS- $b$-P $t$ BA films with topographical patterned poly(dimethylsiloxane) (PDMS) stamps. Imprinting at temperatures above the thermal deprotection temperature of the tert-butyl ester renders the polymer film surfaces' carboxylic acid functional for further derivatization with e.g. biologically relevant molecules. In Chapter 7 scanning thermal lithography (SThL) was introduced as a promising approach for the spatially controlled and highly localized thermal chemical deprotection of surface exposed tert-butyl ester protected carboxylic acid groups in PS- $b$-P $t$ BA films. ${ }^{10}$

PS- $b$-P $t$ BA block copolymer films were recently introduced as robust and versatile (bio)reactive platforms that could be wet chemically or thermally activated prior to aqueous derivatization with biologically relevant molecules. ${ }^{11}$ Despite the robustness of the PS- $b$-P $t$ BA platforms, carboxylic acid functional domains smaller than $\sim 400 \mathrm{~nm} \times \sim 600 \mathrm{~nm}$ were not achieved via $\mathrm{SThL}$. This is ascribed to polymer deformation at temperatures higher than the glass transition temperature applied during writing with SThL. Hence, the domain morphology observed in Chapter 7 
Figure 7.10 is a result of both thermal chemical deprotection as well as thermal mechanical deformation (i.e. rim formation). In addition to SThL on PS- $b$-P $t$ BA films, King and coworkers have reported on the heated probe induced thermal chemical surface functionalization of specially designed, crosslinked polymer films yielding surface exposed carboxylic acid ${ }^{12}$ or amine moieties. ${ }^{13}$ Despite photo crosslinking of the copolymer films, topographic deformations, though smaller compared to those observed for SThL on poly(tetra-hydropyranyl methacrylate) homopolymer films, remained. At the reported high tip velocities $\left(>85 \mu \mathrm{m} \mathrm{s}^{-1}\right)$ and temperatures $\left(>160{ }^{\circ} \mathrm{C}\right)$ used for SThL, formation of rims was observed exhibiting heights $>10 \mathrm{~nm}$.

In order to improve the thermal mechanical stability of SThL, the development of crosslinked polymer films based on tert-butyl ester protected carboxylic acid bearing monomers and di-functional crosslinkers was proposed (Figure 8.1).

A<smiles>C=C(C)C(=O)OC(C)(C)[18O]CCCCCCCCCCCCCCC</smiles>
ethylene glycol dimethacrylate<smiles>C=C(C)C(=O)OCCOC(=O)C(=C)C</smiles>
Irgacure 184 (3 wt\%), UV $(\lambda=306 \mathrm{~nm}, \sim 11 \mathrm{~mW}, 30 \mathrm{~min})$

B<smiles>[B]C(=O)C=C</smiles>
tert-butyl acrylate<smiles>C=CC(=O)OCCCCOC(=O)C=C</smiles>
(c) 1,4 butanediol diacrylate

Figure 8.1: Schematic representation of synthesis of crosslinked polymer films based on A) tert-butyl methacrylate and ethylene glycol dimethacrylate, and B) tert-butyl acrylate and 1.4 butanediol diacrylate, respectively. Typically $20 \mathrm{wt} \%$ of di-functional crosslinker was used.

The two systems developed are based on the free radical photo initiated polymerization of either tert-butyl methacrylate ( $t \mathrm{BMA})$ and ethylene glycol dimethacrylate (EGDMA) or tert-butyl acrylate $(t \mathrm{BA})$ and 1,4 butanediol diacrylate (dBDA) monomer mixtures between two glass cover slides in the presence of a photo initiator. The prepared polymer films are referred to as $\mathrm{MA}_{\mathrm{x}}$ and $\mathrm{A}_{\mathrm{x}}$, in which MA and A stands for methacrylate and acrylate based films, respectively. The number in subscript ( $\mathrm{x}$ ) represents the $\mathrm{wt} \%$ of crosslinker in the monomer mixture (typically $\mathrm{x}$ was $\sim 20 \mathrm{wt} \%$, which corresponds to $\sim 15 \mathrm{~mol} \%$ for both systems). 
In this chapter the applicability of crosslinked $\mathrm{MA}_{\mathrm{x}}$ and $\mathrm{A}_{\mathrm{x}}$ polymer films for sub 100 nanometer thermal chemical surface functionalization with SThL was explored. The differences in thermal deprotection reactions for MA and A films and their subsequent wet chemical grafting with model compounds were unraveled. Furthermore, the effect of AFM cantilever tip radius and tip velocity for SThL on the ultimate resolution was elucidated.

\subsection{Results and discussion}

\subsubsection{Film formation}

$\mathrm{MA}_{20}$ and $\mathrm{A}_{20}$ monomer films between glass microscopy slides were UV polymerized in the presence of Irgacure 184 as photo initiator for 30 minutes. Figure 8.2 shows single reflection ATR-FTIR spectroscopy spectra of the corresponding monomer solutions before polymerization and of the crosslinked polymer films after UV polymerization.
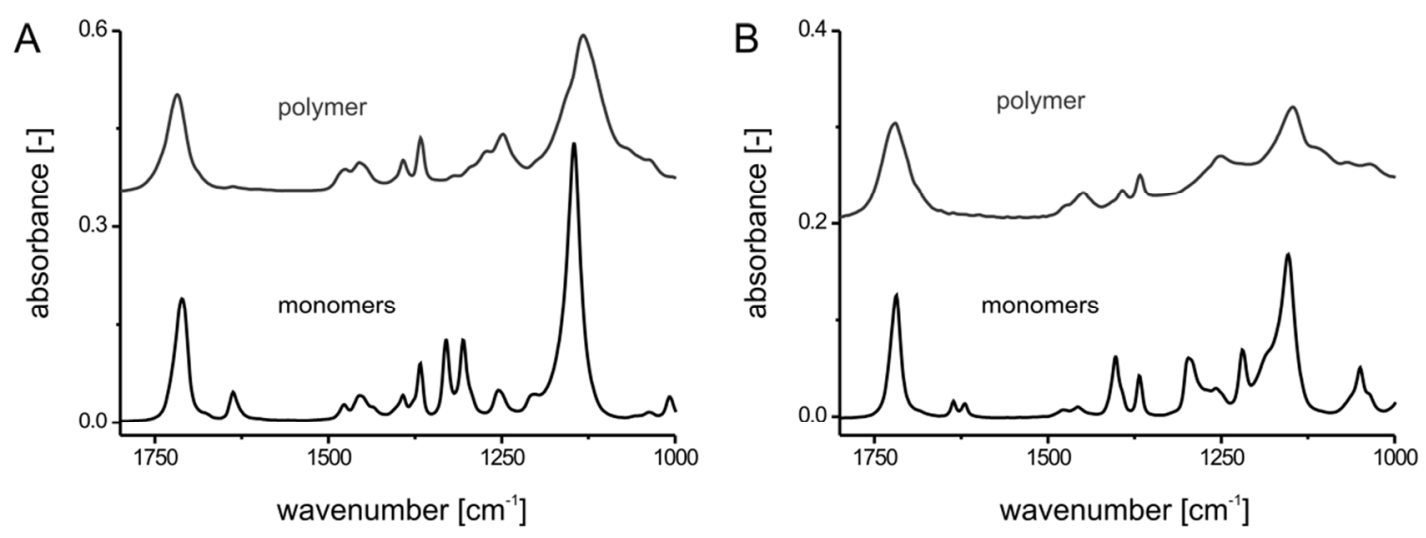

Figure 8.2: Single reflection ATR-FTIR spectra of $M A_{20}(A)$ and $A_{20}(B)$ monomer solutions before and after 30 minutes of UV polymerization. The complete disappearance of the $v_{C=C}\left(\right.$ vinyl) band near $1637 \mathrm{~cm}^{-1}(A)$ and $1620-1636 \mathrm{~cm}^{-1}(B)$ indicates a $100 \%$ conversion of monomer to polymer after 30 minutes of $11 \mathrm{~mW}$ UV radiation for both systems. This is confirmed by the disappearance of the $\delta_{C-H}\left(=\mathrm{CH}_{2}\right)$ absorbances near $1305-1330 \mathrm{~cm}^{-1}(\mathrm{~A})$ and $1409 \mathrm{~cm}^{-1}(B)$.

The formation of glass supported $\mathrm{MA}_{20}$ films is confirmed by the disappearance of the absorbances corresponding to vibrations of $v_{\mathrm{C}=\mathrm{C}}$ (vinyl) at $1635 \mathrm{~cm}^{-1}$ and $\delta_{\mathrm{C}-\mathrm{H}}\left(=\mathrm{CH}_{2}\right)$ at $1330 \mathrm{~cm}^{-1}$ and $1305 \mathrm{~cm}^{-1}$ respectively (Figure 8.2A). The disappearance of the $\delta_{\mathrm{C}-\mathrm{H}}\left(=\mathrm{CH}_{2}\right)$ absorbance at $1409 \mathrm{~cm}^{-1}$ and the $v_{\mathrm{C}=\mathrm{C}}$ (vinyl) absorbances at $1636 \mathrm{~cm}^{-1}$ and $1620 \mathrm{~cm}^{-1}$, respectively, confirms the formation of $\mathrm{A}_{20}$ films on glass substrates (Figure $8.2 \mathrm{~B}$ ). The observed disappearance of the vinyl 
absorbances indicates a complete conversion of monomer to polymer for both the acrylate as well as the methacrylate based polymer films.

\subsubsection{Thermal decomposition reactions in $\mathrm{MA}_{20}$ and $\mathrm{A}_{20}$}

Despite the close resemblance of methacrylate and acrylate monomer structures their thermolysis behavior is known to be quite different. Methacrylates are known to show a higher degree of depolymerization at elevated temperatures compared to acrylates with the same ester substituent. ${ }^{14}$ In Figure 8.3 the proposed reaction mechanisms for $\mathrm{MA}_{20}$ and $\mathrm{A}_{20}$ thermolysis are shown.

Grassie $^{14}$ provided an overview of the primary thermal degradation mechanism observed in methacrylates depending on the number of $\beta$ hydrogen atoms on the ester substituent. The two competing reaction mechanisms are $i$ ) depolymerization and ii) de-esterification with a strong autocatalytic nature followed by the loss of water yielding intramolecular anhydride bonds. In general, the higher the number of $\beta$ hydrogen atoms on the ester substituent, the higher is the contribution of deesterification as the (primary) reaction mechanism upon thermolysis. Tert-butyl esters, having $9 \beta$ hydrogen atoms, are thus mainly subjected to ester decomposition with very little depolymerization upon thermolysis. In another paper Grant and Grassie ${ }^{15}$ reported on the thermal decomposition of poly(tert-butyl methacrylate) P $t \mathrm{BMA}$ at temperatures between $180{ }^{\circ} \mathrm{C}$ and $200{ }^{\circ} \mathrm{C}$. In the initial stage of the thermal decomposition reaction depolymerization yielding tert-butyl methacrylate monomer was found to be the primary reaction. Upon thermal decomposition of tert-butyl ester groups and the subsequent formation of intramolecular anhydride groups, polymer decomposition was inhibited. In contrast to methacrylates, acrylates as a class of polymers typically do not undergo depolymerization reactions. It was reported by Grant and Grassie ${ }^{15}$ that besides the formation of isobutylene and water no monomer traces were observed upon thermolysis of PtBA. De-esterification followed by the formation of intramolecular anhydride groups are the primary thermal decomposition mechanism observed in PtBA (see also Chapter 6 and 7). 


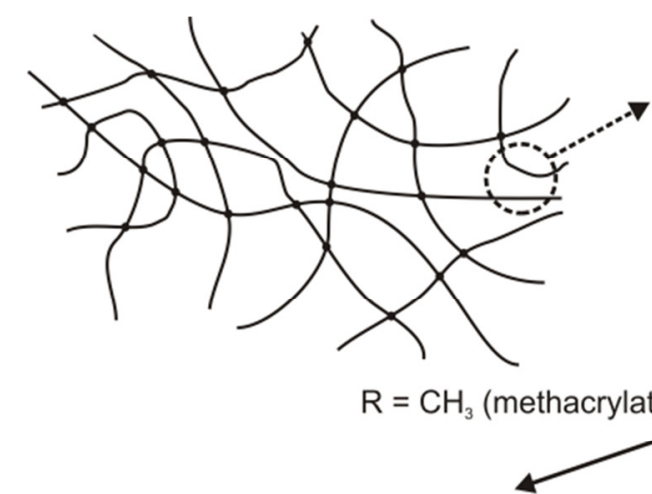

A

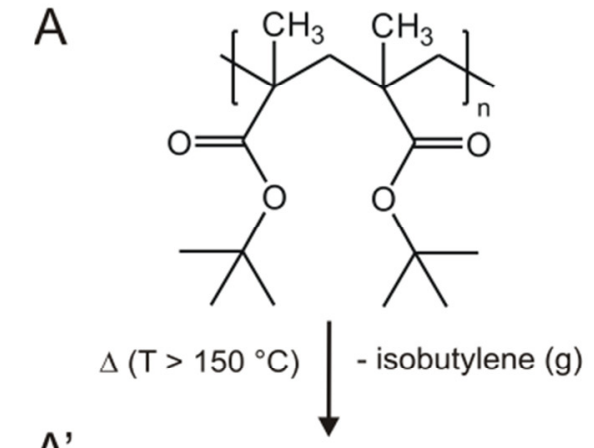

B

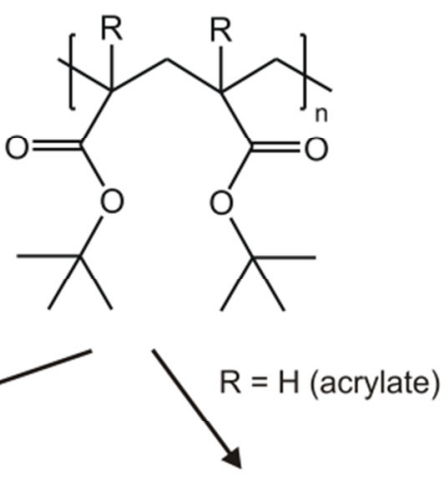

$A^{\prime}$
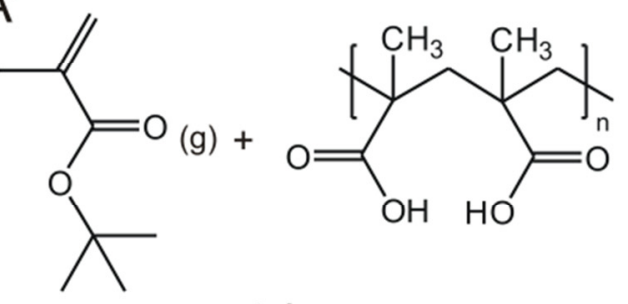

$\mathrm{B}^{\prime}$

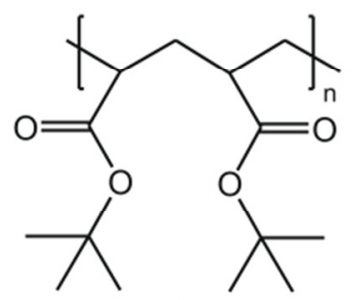

$\left.\Delta\left(\mathrm{T}>150^{\circ} \mathrm{C}\right)\right\rfloor$ - isobutylene $(\mathrm{g})$<smiles>CC(C)(C)C(CC(C(=O)O)C(C)(C)C)C(=O)O</smiles>

$$
+\mathrm{H}_{2} \mathrm{O} \uparrow \mid \begin{aligned}
& \Delta\left(\mathrm{T}>150^{\circ} \mathrm{C}\right) \\
& -\mathrm{H}_{2} \mathrm{O}(\mathrm{g})
\end{aligned}
$$

A"<smiles>CCC1(C)CC(C)(CC(C)(C)C)C(=O)OC1=O</smiles>

B"

$$
+\mathrm{H}_{2} \mathrm{O} \uparrow \mid \begin{aligned}
& \Delta\left(\mathrm{T}>150^{\circ} \mathrm{C}\right) \\
& -\mathrm{H}_{2} \mathrm{O}(\mathrm{g})
\end{aligned}
$$<smiles>CC1CC(CC(C)(C)C)C(=O)OC1=O</smiles>

Figure 8.3: Thermal deprotection mechanism of $M A_{20}(A)$ and $A_{20}(B)$ polymer films. The thermolysis of $M A_{20}$ is initially dominated by depolymerization quantitatively yielding tert-butyl methacrylate. Anhydride formation ( $A$ ") following ester decomposition yielding isobutylene and carboxylic acid moieties ( $\left.A^{\prime}\right)$ inhibits the depolymerization in a later stage of the thermal decomposition reaction. Thermal decomposition of $A_{20}$ initially yields carboxylic acid groups upon the loss of isobutylene ( $\left.B^{\prime}\right)$ followed by the loss of water to form intramolecular anhydride groups ( $B^{\prime \prime)}$. Note: The intramolecular cyclization step (i.e. anhydride formation in $A$ " and $B ")$ is limited in yield due to statistical reasons to $87 \%{ }^{16}$

In Figure 8.4 isothermal thermogravimetric analysis (TGA) data of $\mathrm{MA}_{20}(\mathrm{~A})$ and $\mathrm{A}_{20}(\mathrm{~B})$ are shown. The dashed horizontal lines in Figure 8.4A and 8.4B represent the theoretical residual mass for quantitative loss of isobutylene (upper) and the loss of water (lower). Obviously, thermal decomposition of $\mathrm{MA}_{20}$ for 30 minutes at temperatures above $230{ }^{\circ} \mathrm{C}$ results in a higher mass loss compared to the 
stoichiometric loss of isobutylene and water. This is in line with the occurrence of a depolymerization reaction during the initial stage of the thermal decomposition as discussed above. Thermal decomposition of $\mathrm{A}_{20}$ exhibits essentially quantitative mass loss values in accordance to the loss of isobutylene and water which is in agreement with the thermal decomposition mechanism presented in Figure 8.3. The observed slightly higher residual mass after 30 minutes is presumably the result of inhibition of the autocatalytic thermal ester decomposition as a result of the crosslinked morphology.
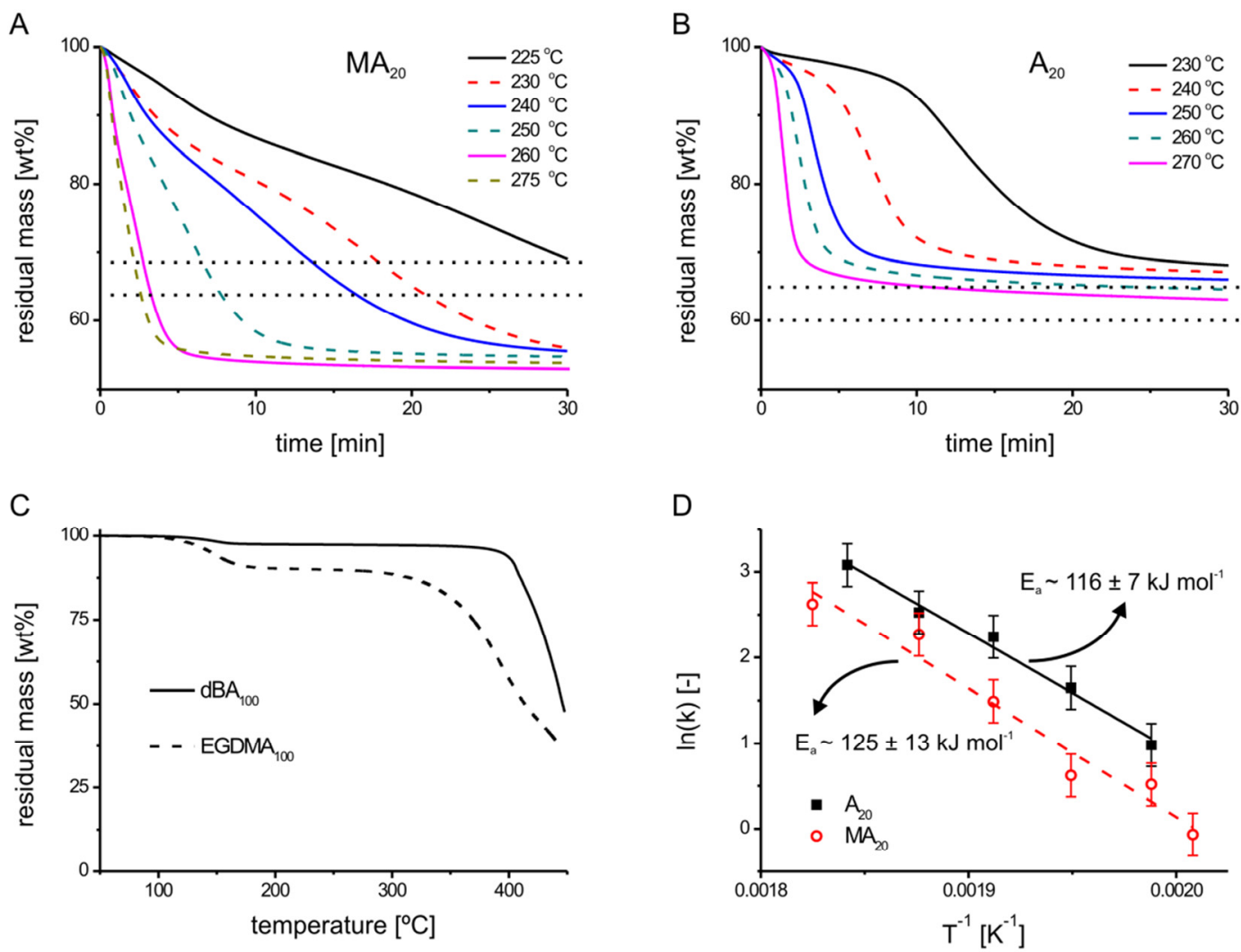

Figure 8.4: Isothermal TGA data of bulk samples of $M A_{20}(A)$ and $A_{20}(B)$ for temperatures above the ester thermal deprotection temperature. Heating from room temperature to the isothermal holding temperatures was applied at a rate of $200^{\circ} \mathrm{C} \mathrm{min}{ }^{-1}$. The horizontal dashed lines represent the theoretical expected weight loss of isobutylene (upper) and subsequently water (lower) upon thermolysis based on the actual polymer compositions. The statistically limited yield of the intramolecular cyclization is taken into account. C) Non isothermal TGA data of bulk polymerized EGDMA and dBDA (at a heating rate of $20{ }^{\circ} \mathrm{C} \mathrm{min}{ }^{-1}$ ). D) Arrhenius plot of zero order reaction rate constants obtained from linear least square fits to the data shown in $A$ and $B$ between a residual mass of 80 to $70 w t \%$ and 90 to $80 w t \%$, respectively.

In Figure 8.4C TGA data of EGDMA and dBDA bulk polymer samples are shown. The acrylate crosslinker is thermally stable up to temperatures of approximately $400{ }^{\circ} \mathrm{C}$. In contrast, the methacrylate crosslinker shows a significant mass loss around $150{ }^{\circ} \mathrm{C}$, which is in agreement with the occurrence of 
depolymerization reactions. The presence of crosslinks in the polymer possibly prevents the completion of polymer decomposition and stabilizes the polymer sample up to temperatures of approximately $320{ }^{\circ} \mathrm{C}$ after which further thermal decomposition occurs.
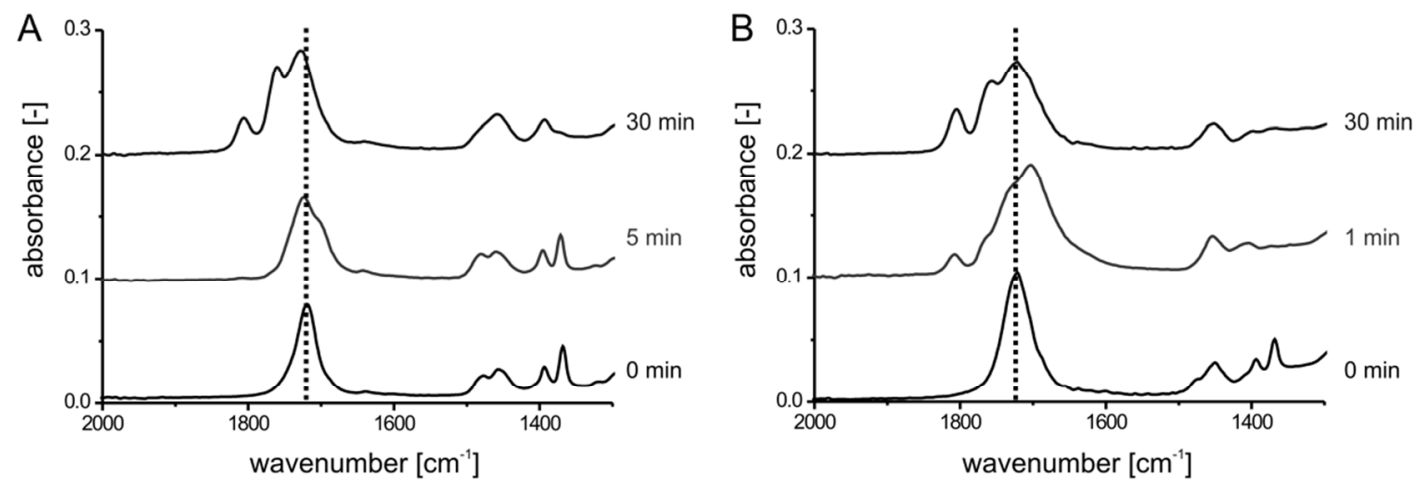

Figure 8.5: Single reflection ATR-FTIR spectra of $M A_{20}(A)$ and $A_{20}(B)$ before and after thermolysis at $250{ }^{\circ} \mathrm{C}$ for times up to 30 minutes. Upon thermolysis the $v_{C=0}$ (ester at $\left.1726 \mathrm{~cm}^{-1}\right)$ absorbance shifts to $1709 \mathrm{~cm}^{-1}$ ( $v_{C=0}$ carboxylic acid) followed by a shift to $1752 \mathrm{~cm}^{-1}$ and $1804 \mathrm{~cm}^{-1}$ corresponding to $v_{\mathrm{C}=\mathrm{O}}$ (anhydride) absorbances. During thermolysis the absorbances corresponding to $\delta_{C-H}$ (tert-butyl methyl) between $1350 \mathrm{~cm}^{-1}$ and $1400 \mathrm{~cm}^{-1}$ disappear. Anhydride formation occurs at lower thermolysis times for the acrylate based system compared the methacrylate based system.

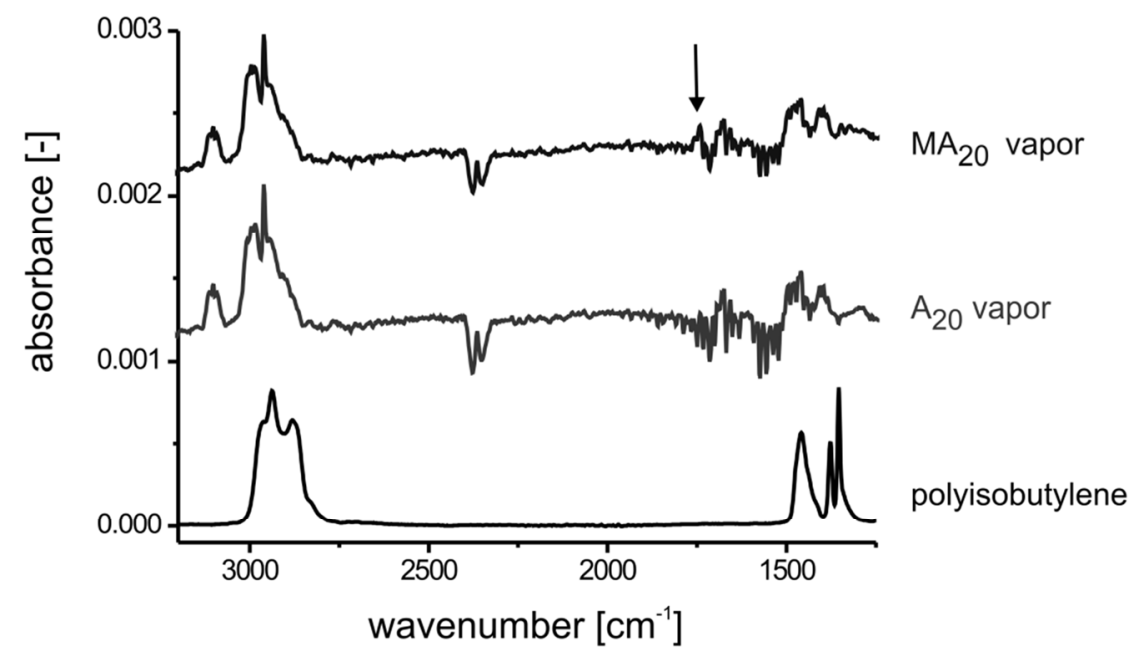

Figure 8.6: Transmission FTIR spectra of gaseous reaction products of $M A_{20}$ and $A_{20}$ heated at $260{ }^{\circ} \mathrm{C}$. For comparison the single reflection ATR-FTIR spectra of poly(isobutylene) is shown (bottom). The similarities of the $M A_{20}$ and $A_{20}$ gaseous reaction product traces with the poly(isobutylene) spectrum are obvious. The presence of $v_{C=C}$ (vinyl) absorbances near $1666 \mathrm{~cm}^{-1}$ and $3094 \mathrm{~cm}^{-1}$ is in agreement with the presence of isobutylene as a reaction product. In addition to isobutylene absorbance the presence of a $v_{C=0}$ (ester at $1726 \mathrm{~cm}^{-1}$ ) absorbance is clearly observed for $M A_{20}$, confirming the loss of monomer (tert-butyl methacrylate). 
Figure 8.5 shows single reflection ATR-FTIR spectra of $\mathrm{MA}_{20}(\mathrm{~A})$ and $\mathrm{A}_{20}(\mathrm{~B})$ bulk polymer samples before and after thermolysis at $250{ }^{\circ} \mathrm{C}$ for different times, respectively (compare Figure 6.3 and Figure 7.3). Before thermal decomposition the $v_{\mathrm{C}=\mathrm{O}}$ (ester) absorbance at $1726 \mathrm{~cm}^{-1}$ is clearly visible (dashed vertical lines). Upon thermolysis the ester stretching absorbance shifts to $1709 \mathrm{~cm}^{-1}$ which corresponds to $v_{\mathrm{C}=\mathrm{O}}$ (carboxylic acid) absorbances followed by a shift to $1752 \mathrm{~cm}^{-1}$ and $1804 \mathrm{~cm}^{-1}$ corresponding to $v_{\mathrm{C}=\mathrm{O}}$ (anhydride) absorbances. In agreement with the disappearance of the ester stretching absorbance, the $\delta_{\mathrm{C}-\mathrm{H}}$ (tert-butyl methyl) absorbance between $1350 \mathrm{~cm}^{-1}$ and $1400 \mathrm{~cm}^{-1}$ disappears, accordingly. The observed shifts in $v_{\mathrm{C}=\mathrm{O}}$ and disappearance of $\delta_{\mathrm{C}-\mathrm{H}}$ (tert-butyl methyl) are in agreement with the FTIR spectra shown in Chapter 6 and Chapter 7 for PS- $b$-P $t$ BA polymer thin films after thermolysis. The residual $v_{\mathrm{C}=\mathrm{O}}$ (ester) absorbances observed in both the $\mathrm{MA}_{20}$, as well as in the $\mathrm{A}_{20}$ spectra are related to the presence of ester groups within the used crosslinkers (see Figure 8.1). ${ }^{17}$ In addition, residual $v_{\mathrm{C}=\mathrm{O}}$ (ester) absorbances for $\mathrm{A}_{20}$ might partially be related to residual tert-butyl ester group absorbances, which would explain the residual mass observed in Figure $8.4 \mathrm{~B}$ being slightly higher compared to the theoretical quantitative yield of isobutylene and water. Furthermore it was observed that anhydride formation occurs at lower thermal decomposition times for $\mathrm{A}_{20}$ samples compared to $\mathrm{MA}_{20}$ samples (compare middle FITR spectra in Figures $8.5 \mathrm{~A}$ and $8.5 \mathrm{~B}$ ). This is in line with the competitive thermal decomposition reactions in $\mathrm{MA}_{20}$ samples during the initial stage of thermal decomposition (i.e. depolymerization $v s$. de-esterification).

Figure 8.6 shows transmission FTIR spectra of the gaseous reaction products of $\mathrm{MA}_{20}$ and $\mathrm{A}_{20}$ samples upon thermolysis at $260{ }^{\circ} \mathrm{C}$. The spectra resemble the poly(isobutylene) spectrum with the difference that the polymer lacks the presence of $v_{\mathrm{C}=\mathrm{C}}$ (vinyl) (monomer) absorbances near $1666 \mathrm{~cm}^{-1}$ and $3094 \mathrm{~cm}^{-1}$. The presence of tert-butyl methacrylate monomer in the $\mathrm{MA}_{20}$ reaction products is evident from the presence of a $\mathrm{v}_{\mathrm{C}=\mathrm{O}}$ (ester) absorbance at $1726 \mathrm{~cm}^{-1}$. The presence of water absorbances could partly be ascribed to the formation of intramolecular anhydride groups or to the variation of the water content compared to the background spectrum. Hence from these measurements no quantitative information could be obtained.

To obtain the apparent ester decomposition activation energy, the isothermal TGA data between 80 to $70 \%$ and 90 to $80 \%$ residual mass for $\mathrm{MA}_{20}$ and $\mathrm{A}_{20}$ was treated as the autocatalytic thermal decomposition of tert-butyl ester to yield carboxylic acid groups. Autocatalytic decomposition reactions typically show zero order reaction kinetics. Linear least square fits to the data within the mentioned residual mass ranges confirmed the zero order reaction kinetics for both systems 
under investigation. In this treatment, the following assumptions were made: i) Depolymerization occurs solely during the initial stage of $\mathrm{MA}_{20}$ decomposition. ii) The used crosslinkers do not interfere with the thermal ester decomposition reactions. iii) In the stated residual mass loss ranges, carboxylic acid groups and isobutylene are the primary reaction products.

Figure $8.4 \mathrm{D}$ shows a plot of the values of the zero order reaction rate constants versus $\mathrm{T}^{-1}$ according to the Arrhenius equation:

$k=A e^{-\frac{E_{a}}{R T}}$

In which $k$ is the reaction rate constant, $A$ is the pre-exponential factor, $R$ is the gas constant $\left(8.314 \mathrm{~J} \mathrm{~K}^{-1} \mathrm{~mol}^{-1}\right.$ ) and $T$ denotes the temperature $(\mathrm{K})$. The apparent activation energies $\left(E_{a}\right)$ for $\mathrm{MA}_{20}$ and $\mathrm{A}_{20}$ were calculated to be $125 \pm 13 \mathrm{~kJ} \mathrm{~mol}^{-1}$ and $116 \pm 7 \mathrm{~kJ} \mathrm{~mol}^{-1}$, respectively. These numbers are in good agreement with results reported by others for the activation energy of tert-butyl ester decomposition of PtBA. ${ }^{18}$

\subsubsection{Derivatization of thermally deprotected $\mathrm{MA}_{20}$ and $\mathrm{A}_{20}$ bulk samples}

Having confirmed the thermal decomposition mechanism with TGA and FTIR spectroscopy for both $\mathrm{MA}_{20}$ and $\mathrm{A}_{20}$ the chemical functionality of the in situ formed carboxylic acid and anhydride groups was investigated with fluorescence microscopy. Fluoresceinamine was covalently immobilized to thermally functionalized $\mathrm{MA}_{20}$ and $\mathrm{A}_{20}$ surfaces according to well known wet chemical grafting procedures in which the carboxylic acid groups were EDC/NHS activated.

Figure 8.7A shows the normalized fluorescence microscopy counts of fluoresceinamine modified $\mathrm{MA}_{20}$ and $\mathrm{A}_{20}$ samples before and after thermal activation at $250{ }^{\circ} \mathrm{C}$ for 30 seconds and 60 seconds, respectively. With increasing thermal activation time the normalized intensity increases for both $\mathrm{MA}_{20}$ as well as for $\mathrm{A}_{20}$ samples. Fluoresceinamine modified $\mathrm{MA}_{20}$ samples show a lower emission compared to $\mathrm{A}_{20}$ fluoresceinamine modified samples for the same thermal activation time and temperature. This is ascribed to the difference in thermal decomposition mechanism for $\mathrm{MA}_{20}$ and $\mathrm{A}_{20}$ as discussed earlier. The initial depolymerization reaction for the $\mathrm{MA}_{20}$ sample results in a significantly lower degree of functionalization as compared to the $\mathrm{A}_{20}$ thermally functionalized sample in which depolymerization reactions are absent. Despite the different reaction mechanisms both samples were successfully thermally activated and subsequently modified with fluoresceinamine following 
EDC/NHS activation of the in situ formed carboxylic acid moieties. Thermally activated samples without EDC/NHS activation of the carboxylic acid groups did not show significant emission after immersion in a fluoresceinamine solution.

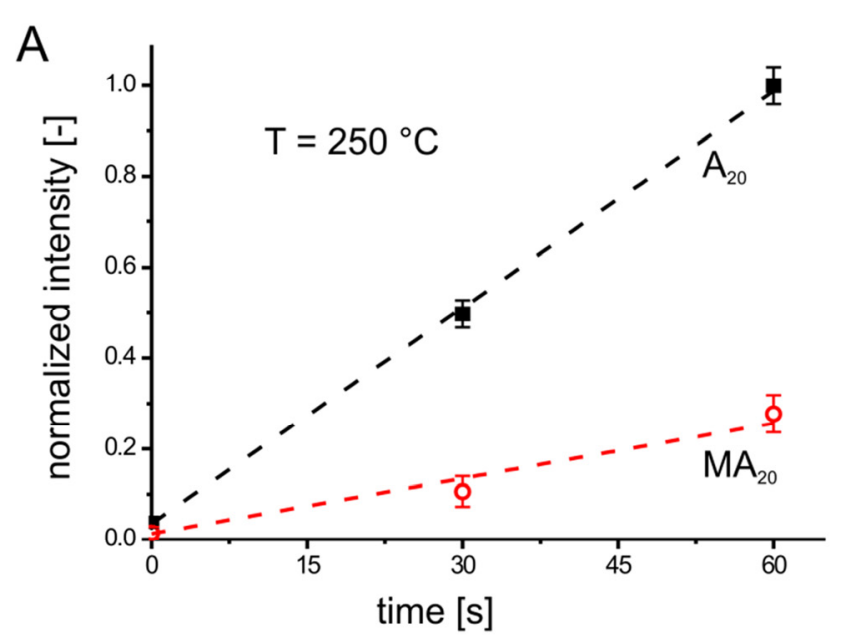

B
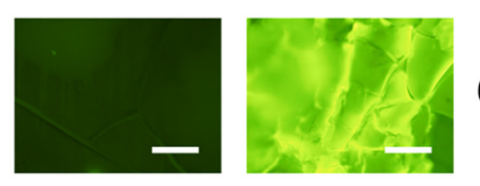
$60 \mathrm{~s}$
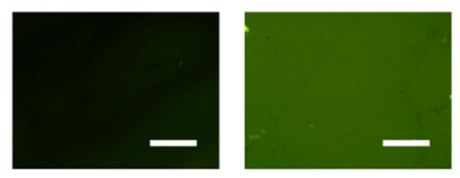

$30 \mathrm{~s}$

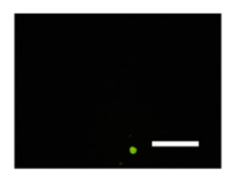

$\mathrm{MA}_{20}$

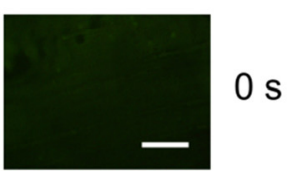

$\mathrm{A}_{20}$

Figure 8.7: A) Normalized fluorescence microscopy counts as a function of thermolysis time at $250{ }^{\circ} \mathrm{C}$ for $M A_{20}$ (open red circles) and $A_{20}$ (filled black squares) samples covalently modified with fluoresceinamine $\left(\lambda_{\text {ex }} \approx 496 \mathrm{~nm}\right.$ and $\lambda_{\text {em }} \approx 520 \mathrm{~nm}$ ) following EDC/NHS activation. A high pressure Mercury lamp was used for excitation ( $\lambda_{e x} 460-490 \mathrm{~nm}$ ). The dashed lines are a guide for the eye. In $B$ fluorescence microscopy images of the respective samples are shown. The scale bars in B represent $200 \mu \mathrm{m}$.

\subsubsection{Improved thermal mechanical properties for SThL}

Having established the thermal activation mechanism for both $\mathrm{MA}_{20}$ and $\mathrm{A}_{20}$ samples, as well as the successful surface derivatization with fluoresceinamine following EDC/NHS activation of the in situ formed carboxylic acid groups, the performance of $\mathrm{MA}_{20}$ and $\mathrm{A}_{20}$ films during SThL was investigated and compared to PS- $b$-P $t$ BA films discussed in Chapter 7. Figure 8.8 shows contact mode AFM height images (A-C, left) of $\mathrm{MA}_{20}, \mathrm{~A}_{20}$ and a PS- $b$-P $t$ BA polymer film after SThL of a square or set of squares at probe tip temperatures $\left(T_{t i p}\right)$ above the thermal deprotection temperature of the tert-butyl ester. The absence of thermomechanical surface deformations on the crosslinked polymer films is obvious, especially when compared with the micrometer scale deformations observed (i.e. rims) for the block copolymer film (compare Figure 7.10). Interestingly, after SThL ( $\mathrm{T}_{\text {tip }}$ at $260{ }^{\circ} \mathrm{C}, v_{\text {tip }}$ of $2 \mu \mathrm{m} \mathrm{s}^{-1}$ ) on the $\mathrm{MA}_{20}$ surface $\mathrm{a} \sim 15$ nanometer deep shallow depression was observed without the formation of surrounding rims. More fascinating, after SThL on $\mathrm{A}_{20}$ surfaces no signs of topographical changes were observed for $1 \mu \mathrm{m} \times 1 \mu \mathrm{m}$ squares prepared with $T_{\text {tip }}$ ranging from $260{ }^{\circ} \mathrm{C}$ to $280{ }^{\circ} \mathrm{C}$ and $v_{\text {tip }}$ between $2 \mu \mathrm{m} \mathrm{s}^{-1}$ to $6 \mu \mathrm{m} \mathrm{s}^{-1}$. 

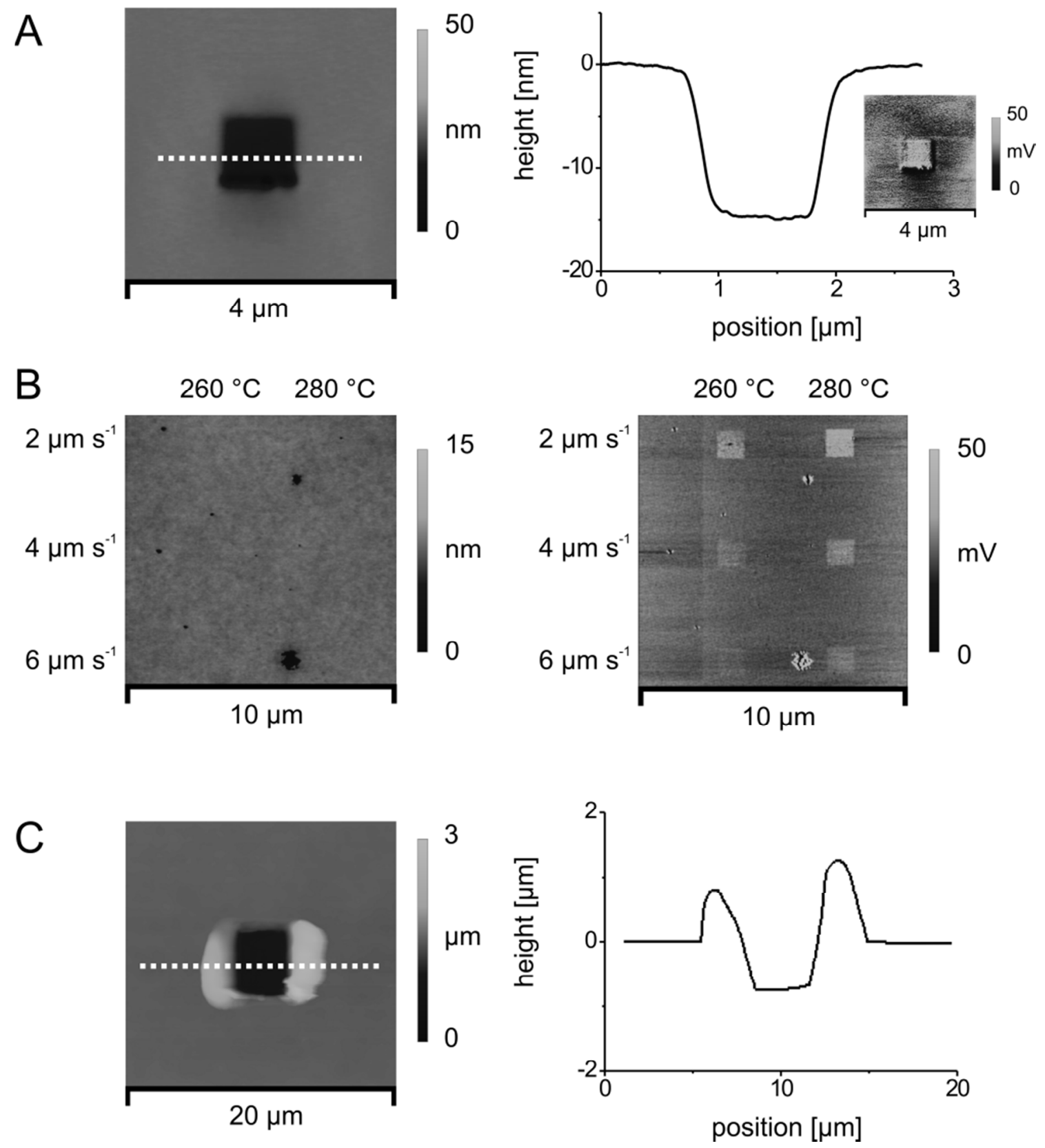

Figure 8.8: A) Contact mode AFM height image (left) of $M A_{20}$ after SThL of a $2 \mu m \times 2 \mu m$ square with $T_{\text {tip }}$ at $260{ }^{\circ} \mathrm{C}$ and a $v_{\text {tip }}$ of $2 \mu \mathrm{m} \mathrm{s}^{-1}$. On the right the corresponding cross-section along the white dashed line is shown. The inset shows the corresponding lateral force image. B) Contact mode AFM height (left) and lateral force (right) images of $A_{20}$ after SThL of $1 \mu \mathrm{m} \times 1 \mu \mathrm{m}$ squares with $T_{\text {tip }}$ at $260{ }^{\circ} \mathrm{C}$ and $280{ }^{\circ} \mathrm{C}$ for writing speeds of $2 \mu \mathrm{m} \mathrm{s}{ }^{-1}, 4 \mu \mathrm{m} \mathrm{s} \mathrm{s}^{-1}$ and $6 \mu \mathrm{m} \mathrm{s}^{-1}$, respectively. C) Contact mode AFM height image of a silicon supported PS-b-PtBA block copolymer film (left) after SThL of a $5 \mu m \times 5 \mu m$ square with $T_{\text {tip }} 265^{\circ} \mathrm{C}$ and a $v_{\text {tip }}$ of $5 \mu \mathrm{m} \mathrm{s}^{-1}$. On the right the corresponding cross-section along the white dashed line is shown. The force feedback loop maintained a contact load during SThL of approximately $10 \mathrm{nN}, 30 \mathrm{nN}$ and $10 \mathrm{nN}$ for the results shown in $A, B$ and C, respectively. 
In addition to the observed shallow surface depression after $\mathrm{SThL}$ on $\mathrm{MA}_{20}$ films, an increase in lateral force signal was observed in the areas thermochemical activated with SThL (inset Figure 8.8A right), indicating the formation of a spatially controlled more hydrophilic surface area. ${ }^{19}$ The observed increase in hydrophilicity is to be expected upon the thermal decomposition of surface exposed tert-butyl esters yielding carboxylic acid groups. The formation of shallow surface depressions is ascribed to the loss of $t$ BMA (g), resulting in localized partial degradation of the network structure. Despite the lack of topographical changes on $A_{20}$ films after SThL of $1 \mu \mathrm{m} \times 1 \mu \mathrm{m}$ squares the thermochemically activated areas show an increase in lateral force signal (i.e. increased hydrophilicity, Figure $8.8 \mathrm{~B}$ right). This is a strong indication for the localized conversion of tert-butyl esters to carboxylic acid groups in analogy with $\mathrm{MA}_{20}$ films. The observed lateral force signal as a qualitative measure of tert-butyl ester to carboxylic acid conversion shows an increase in conversion for higher $T_{t i p}$ and slower scan speeds (longer thermolysis times), as expected. The observed changes in topography and surface hydrophilicity are in agreement with the earlier discussed thermal decomposition mechanisms for $\mathrm{MA}_{20}$ and $\mathrm{A}_{20}$.

A

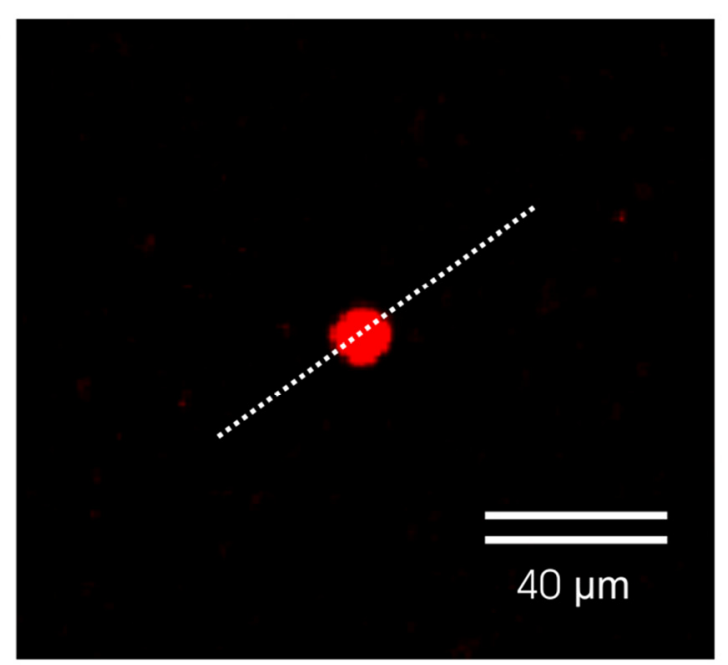

$\mathrm{B}$

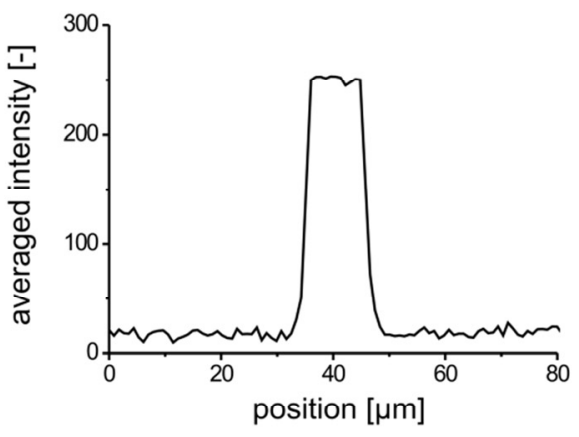

C

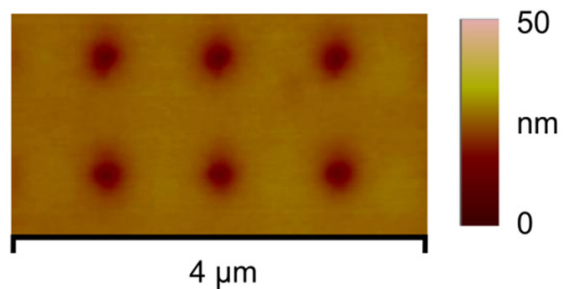

Figure 8.9: A) Confocal fluorescence microscopy image $\left(\lambda_{e x} \approx 488 \mathrm{~nm}\right.$ ) of a $10 \mu \mathrm{m} \times 10 \mu \mathrm{m}$ square ( $x$-y separation $1100 \mathrm{~nm}$ ) prepared on an $A_{15}$ film by $S T h L$ through indenting a $10 \times 10$ point array ((x-y separation $1100 \mathrm{~nm})$ with a heated probe $\left(T_{\text {tip }} 280{ }^{\circ} \mathrm{C}\right)$, subsequently chemically grafted with amino functionalized eFluor ${ }^{\circledR} 605$ quantum dots $\left(\lambda_{\mathrm{em}} \approx 605 \mathrm{~nm}\right)$ utilizing EDC/NHS activation. The probe contact load was $\sim 20 \mathrm{nN}$ while the contact time of the heated probe for every point was 1 second. B) Intensity profile along the white dashed line in A. C) Contact mode AFM height image of a part of the freshly prepared dot array. 
Figure 8.9 shows a confocal fluorescence microscopy image of a $10 \times 10$ array of pits on a $\mathrm{A}_{15}$ film that were thermochemically activated through indenting a $10 \times 10$ point array with $T_{\text {tip }}$ at $280{ }^{\circ} \mathrm{C}$ and a tip contact time of 2 seconds. Subsequently the produced pits were covalently modified with amine functionalized eFluor ${ }^{\circledR} 605$ quantum dots (red emission) following well known EDC/NHS chemistry. Since each pit measures approximately $420 \mathrm{~nm}$ by $470 \mathrm{~nm}$ in size, they could not be resolved with fluorescence microscopy. The full width half maximum (FWHM) of the intensity profile (Figure 8.9B) is approximately $11 \mu \mathrm{m}$, which is in good agreement with the overall size of the prepared array $(\sim 10.4 \mu \mathrm{m})$. The clear fluorescence emission detected indicates that the covalent modification with the quantum dots was very efficient. In addition, it points towards the quasi-3D nature of polymer film platforms as (bio)reactive interfaces. ${ }^{11}$

Despite several attempts, fluorescence emission from $\mathrm{MA}_{20}$ films covalently modified with fluoresceinamine was not observed in fluorescence microscopy. This is ascribed to the short thermal deprotection times used for SThL, with depolymerization being the primary reaction mechanism. Hence the yield of surface exposed carboxylic acid groups available for fluoresceinamine functionalization was too low to be detected with fluorescence microscopy. Another explanation that cannot be ruled out at the moment is that depolymerization was the only reaction occurring. However the use of longer SThL times did not improve the observed results. Interestingly, $15 \mu \mathrm{m} \times 15 \mu \mathrm{m}$ squares thermochemical activated via $\mathrm{SThL}\left(T_{\text {tip }}\right.$ at $250{ }^{\circ} \mathrm{C}$ with $\left.v_{t i p} 15 \mu \mathrm{m} \mathrm{s}^{-1}\right)$ on EGDMA crosslinked $t$ BA polymer films (20wt\% EGDMA) were successfully covalently modified with fluoresceinamine as was concluded from the clear green emission observed. The intensity profile had a FWHM of $15 \mu \mathrm{m}$ corresponding very well to the size of the prepared squares.

These results demonstrate that understanding the thermal deprotection mechanism at short time scales is important for the development of nanometer sized functional surface domains with SThL. In addition, the enhanced understanding might result in the development of improved platforms for SThL. For instance, in order to use methacrylate based polymer platforms without the undesired depolymerization reaction being dominant at the very low thermal decomposition time scales used in SThL, one might consider the use of an ester substituent that decomposes at temperatures below which depolymerization reactions occur. King and coworkers ${ }^{12,13}$ have successfully incorporated ester substituents based on tetrahydropyranyl in methacrylate based crosslinked platforms for SThL. In subsequent wet chemical grafting reactions they have demonstrated the wide variety in chemical functionalities 
that could possibly be incorporated in these films. However, their interpretation that the over $100 \mathrm{~nm}$ deep surface depressions observed in P $t$ BMA homopolymer films after SThL could solely be ascribed to thermomechanical surface deformation seems now questionable. In the most extreme scenario as we have demonstrated above (Figure 8.8A) it could solely be explained by depolymerization. However since the thermal deprotection temperature was much higher than the glass transition temperature of the PtBMA homopolymer, it is most likely a combination of both processes.

\subsubsection{SThL on MA20 samples}

Figure 8.10 shows tapping mode images acquired with super sharp silicon tapping mode tips (nominal tip radius $<10 \mathrm{~nm}$ ) and corresponding horizontal crosssections of grooves prepared with $\mathrm{SThL}$ on $\mathrm{MA}_{20}$ films as a function of the tip velocity (left to right) and $T_{\text {tip }}$ (top to bottom). The tip radius $\left(r_{t i p}\right)$ of the heated probe used for writing lines in $\mathrm{MA}_{20}$, determined via scanning an array of sharp tips, ${ }^{20}$ was calculated to be approximately $40 \mathrm{~nm}$. Figure 8.11 shows the measured groove width (A) and depth (B) as a function of $T_{\text {tip }}$ and tip velocity $\left(v_{t i p}\right)$ typically observed for SThL on $\mathrm{MA}_{20}$ samples. $T_{\text {tip }}$ was in the range of $220{ }^{\circ} \mathrm{C}$ up to $260{ }^{\circ} \mathrm{C}$ and $v_{\text {tip }}$ was between $0.2 \mu \mathrm{m} \mathrm{s}^{-1}$ and $1.5 \mu \mathrm{m} \mathrm{s}^{-1}$.
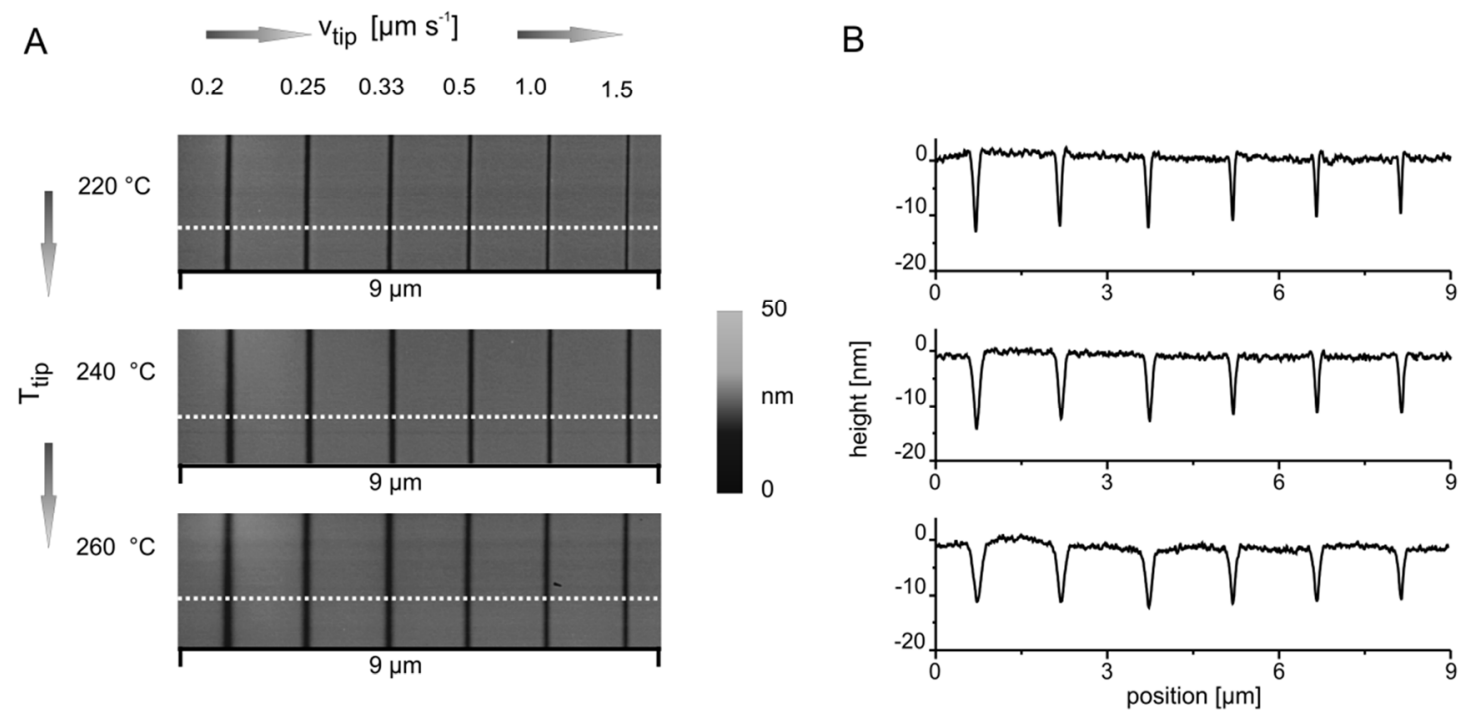

Figure 8.10: A) Tapping mode AFM height images of $M A_{20}$ after SThL of $4 \mu \mathrm{m}$ long lines written with $T_{\text {tip }}$ at $220{ }^{\circ} \mathrm{C}, 240{ }^{\circ} \mathrm{C}$ and $260{ }^{\circ} \mathrm{C}$ at writing speeds ranging between $0.2 \mu \mathrm{m} \mathrm{s}{ }^{-1}$ and $1.5 \mu \mathrm{m} \mathrm{s}^{-1}$. In $B$ the corresponding cross-sections along the white dashed lines are shown. Super sharp silicon tips were used to acquire the tapping mode images.

From Figures 8.10 and $8.11(\mathrm{~A}-\mathrm{B})$ it is clear that with decreasing $T_{\text {tip }}$ and increasing $v_{\text {tip }}$ the groove width and depth decreased from $\sim 307 \mathrm{~nm}$ to $\sim 101 \mathrm{~nm}$ and 
$\sim 13.7 \mathrm{~nm}$ to $\sim 9.8 \mathrm{~nm}$, respectively. This is in agreement with the expected conversion of $\mathrm{MA}_{20}$ upon thermal decomposition being lower for shorter reaction times and lower temperatures (compare Figure 8.4).

Analysis of the volume loss as a function of the averaged tip contact time for the different $T_{\text {tip }}$ used is presented in Figure 8.11C. For averaged contact times ranging between 40 to 200 milliseconds the volume loss was found to be linear in time. Under the assumption that the volume loss is directly proportional to the conversion of the thermal decomposition reaction, yielding presumably mainly $t$ BMA monomer and small amounts of isobutylene, this is in accordance to zero order reaction kinetics. In Figure 8.11D the corresponding Arrhenius plot is shown. The apparent activation energy for groove formation was calculated according to formula (1) to be $45 \pm 3 \mathrm{~kJ}$ $\mathrm{mol}^{-1}$.

A

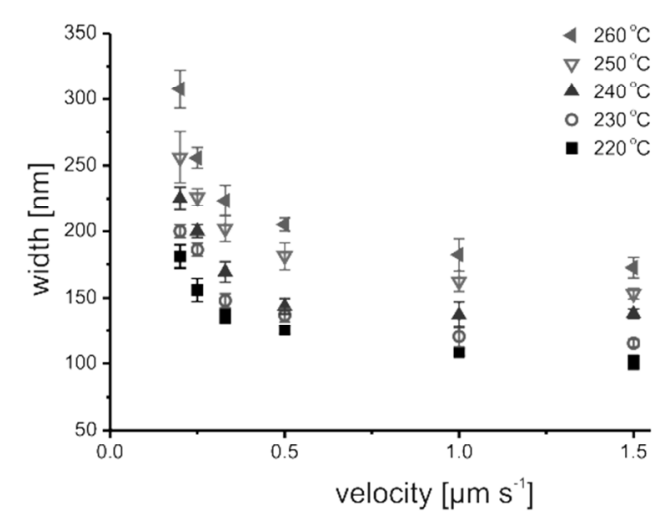

C

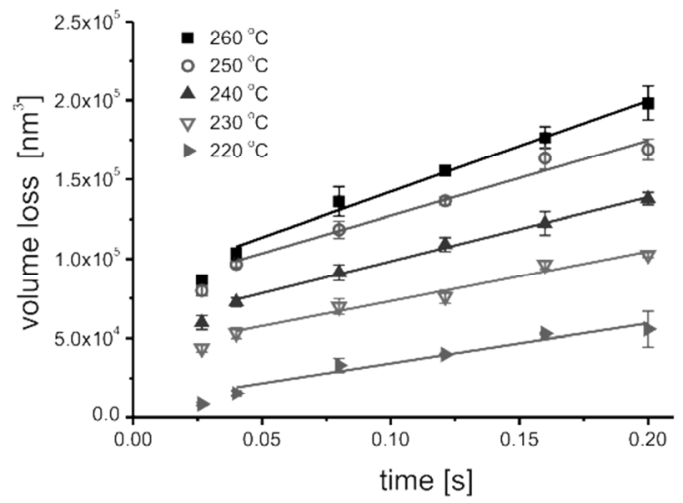

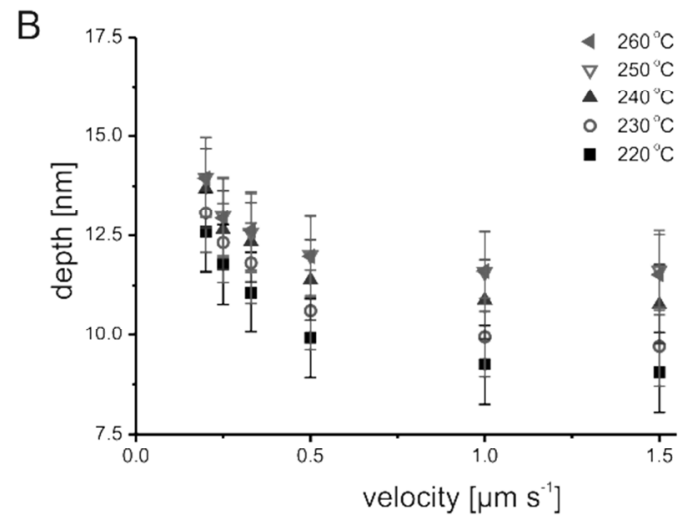

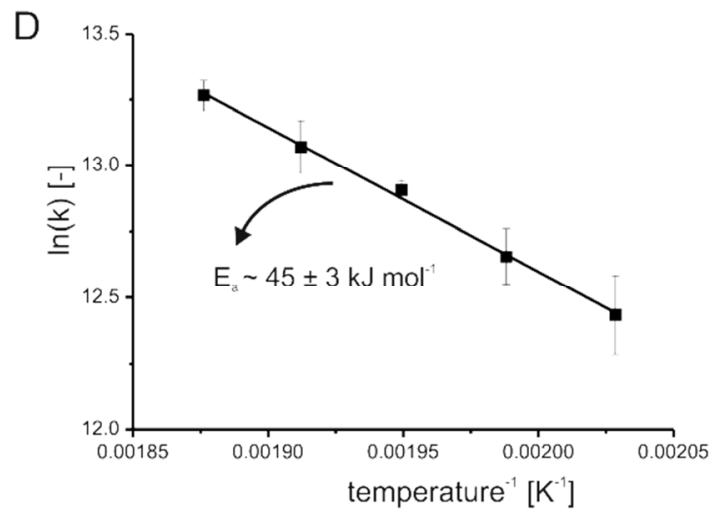

Figure 8.11: Width $(A)$ and depth $(B)$ of lines prepared by $S T h L$ in $M A_{20}$ as a function of the tip velocity for various tip temperatures. Lines of $4 \mu \mathrm{m}$ in length were written with a contact load of $\sim 10 \mathrm{nN}$. C) Volume loss as a function of the tip contact time. The solid lines correspond to linear least square fits of the data, corresponding to zero order reaction kinetics. D) Arrhenius plot of the zero order reaction rate constants obtained from the linear fits in $C$. The solid line represents a linear least square fit to the data.

This significantly lower value of the activation energy compared to the tert-butyl ester deprotection reaction further strengthens the assumption that depolymerization is the primary reaction during $\mathrm{SThL}$ on $\mathrm{MA}_{20}$ for the corresponding low reaction times 
used. The apparent activation energy of $\mathrm{MA}_{20}$ between 98 and $95 \mathrm{wt} \%$ residual mass of the TGA data presented in Figure 8.4A was calculated to be $112 \pm 11 \mathrm{~kJ} \mathrm{~mol}^{-1}$, representing a part of the thermal decomposition reaction in which depolymerization is assumed to be the primary reaction mechanism. However SThL is performed in air atmosphere whereas TGA was performed under nitrogen. For comparison, the oxidative thermal degradation of poly(methyl methacrylate) (PMMA), yielding monomer in quantitative amounts, was reported to have an activation energy of $\sim 64 \mathrm{~kJ} \mathrm{~mol}^{-1}$ whereas in the same study the activation energy for thermal decomposition of PMMA in nitrogen atmosphere was reported to be $\sim 233 \mathrm{~kJ} \mathrm{~mol}^{-1}$, respectively. $^{21}$

The calibrated $T_{t i p}$ used for the calculation of the apparent activation energy for groove formation with SThL in $\mathrm{MA}_{20}$ films $\left(45 \pm 3 \mathrm{~kJ} \mathrm{~mol}^{-1}\right)$ is assumed to be the isothermal reaction temperature in close proximity of the tip-sample contact point. However as already pointed out in Chapter 4 a sharp temperature gradient can be expected within radial distances of approximately $1 \mu \mathrm{m}$ from the tip-surface contact point. Lowering the set $T_{\text {tip }}$ temperature, by e.g. $40{ }^{\circ} \mathrm{C}$, to get a more realistic effective reaction temperature, will decrease the calculated apparent activation energy for groove formation with no more than $6.5 \mathrm{~kJ} \mathrm{~mol}^{-1}$.

\subsubsection{SThL on $A_{20}$ films}

Based on the relatively higher activation energy values determined for the tert-butyl ester decomposition compared to depolymerization, we expect to significantly decrease the pattern sizes prepared with SThL on $\mathrm{A}_{20}$ films in which no depolymerization reactions were observed. Figure 8.12 shows AFM contact mode and lateral force mode images of a $12 \times 12$ array of dots (x-y separation $550 \mathrm{~nm}$ ) prepared by indenting with a heated tip $\left(T_{t i p} \sim 280{ }^{\circ} \mathrm{C}\right.$, probe contact time 2 seconds) on an $\mathrm{A}_{20}$ film. Pits with an increased lateral force signal were prepared which is in accordance with the formation of surface exposed carboxylic acid groups (more hydrophilic) within these domains. The pit diameter was estimated from the lateral force images to be $60 \pm 8 \mathrm{~nm}$, which is about 1.5 times of the approximate tip radius of the used probe $(\sim 40 \mathrm{~nm})$. Despite the much higher contact time compared to the averaged contact times used for the grooves formed in $\mathrm{MA}_{20}$ films (compare Figures 8.10 and 8.11) the thermochemically activated domain size of the individual pits in $\mathrm{A}_{20}$ films is significantly reduced. 

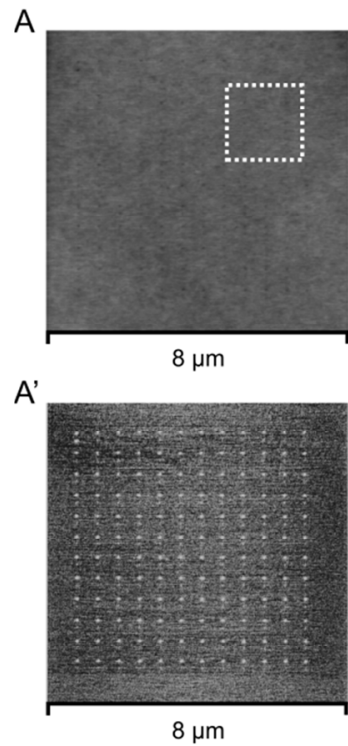

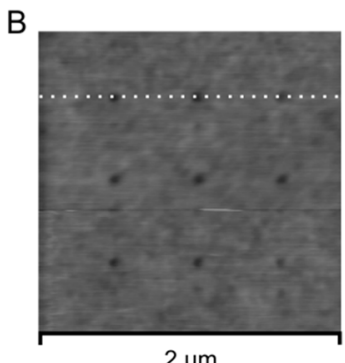

$B^{\prime}$

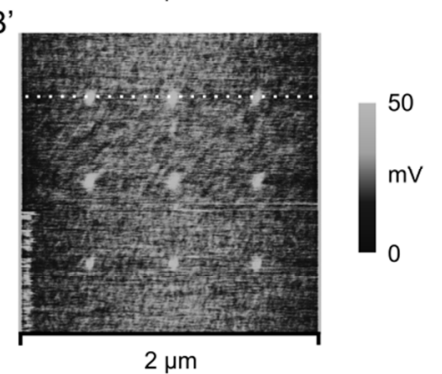

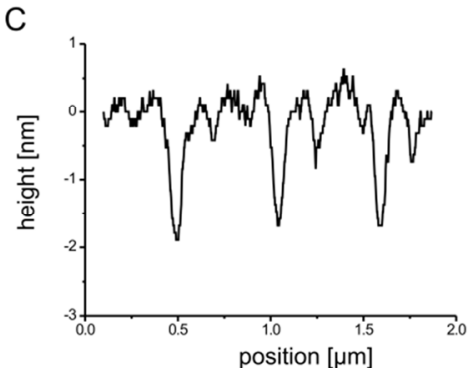

$C^{\prime}$

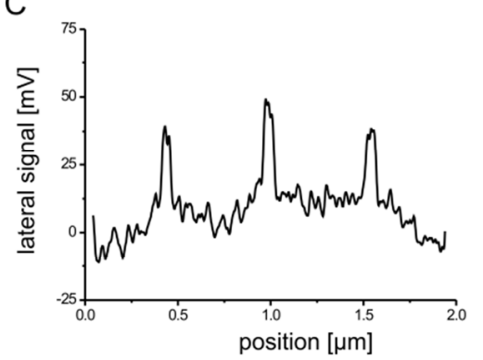

Figure 8.12: Contact mode AFM height images ( $A$ and $B$ ) of an 12 by 12 array prepared via SThL with $T_{\text {tip }} 280^{\circ} \mathrm{C}$ and probe contact times of 2 seconds while maintaining a contact force of $\sim 5 \mathrm{nN}$. Lateral force images of the respective areas are shown in ( $A^{\prime}$ and $B^{\prime}$ ). In $C$ and $C^{\prime}$ the cross-sections along the white dashed lines are shown, respectively.

In order to compare $\mathrm{SThL}$ on $\mathrm{MA}_{20}$ and $\mathrm{A}_{20}$ films, lines were written with identical settings for SThL on both films. $T_{\text {tip }}$ was set to $280{ }^{\circ} \mathrm{C}$ and a tip velocity of $1 \mu \mathrm{m} \mathrm{s}^{-1}$ was used. Furthermore the role of the size of the tip radius on the pattern size was elucidated. The results are shown in Table 8.1, which also includes the ultimate resolution obtained for $\mathrm{SThL}$ on $\mathrm{MA}_{20}$ and $\mathrm{A}_{20}$ films with empirically determined optimal settings.

Table 8.1: Measured line width and depth values for $M A_{20}$ and $A_{20}$ films after SThL with probes possessing a different tip radius. In addition, the optimized settings for achieving the highest spatial resolution with SThL on $M A_{20}$ and $A_{20}$ films are shown.

\begin{tabular}{|c|c|c|c|c|c|c|}
\hline Film & $r_{\text {tip }}[\mathrm{nm}]$ & $\mathrm{T}_{\text {tip }}\left[{ }^{\circ} \mathrm{C}\right]$ & $\mathrm{F}[\mathrm{nN}]$ & $\mathrm{v}\left[\mu \mathrm{m} \mathrm{s}^{-1}\right]$ & width $[\mathrm{nm}]^{\mathrm{a}}$ & depth [nm] \\
\hline $\mathrm{MA}_{20}$ & 40 & 280 & $\sim 20$ & 1 & $412 \pm 37$ & $10.8+-1$ \\
\hline $\mathrm{MA}_{20}$ & 100 & 280 & $\sim 20$ & 1 & $781 \pm 70$ & $13+-1$ \\
\hline $\mathrm{A}_{20}$ & 40 & 280 & $\sim 20$ & 1 & $40 \pm 4^{b}$ & n.d. \\
\hline $\mathrm{A}_{20}$ & 100 & 280 & $\sim 20$ & 1 & $80 \pm 7^{b}$ & n.d. \\
\hline $\mathrm{MA}_{20}$ & 40 & 240 & $\sim 30$ & 500 & $83 \pm 7^{c}$ & $<1$ \\
\hline $\mathrm{A}_{20}$ & 40 & 280 & $\sim 75$ & 5 & $21 \pm 2^{b}$ & n.d \\
\hline
\end{tabular}

${ }^{a}$ No tip deconvolution was applied. ${ }^{b}$ Measured from lateral force images. ${ }^{c}$ Signs of thermomechanical deformations were observed. 
From Table 8.1 it is clear that for the identical SThL settings used the obtained line width in $\mathrm{MA}_{20}$ is approximately 10.3 and 9.8 times higher compared to line formation of lines on $\mathrm{A}_{20}$ for $r_{\text {tip }}$ being $\sim 40 \mathrm{~nm}$ and $\sim 100 \mathrm{~nm}$, respectively. Furthermore, the increase in $r_{\text {tip }}$ with a factor of 2.5 resulted in an increase in observed line widths of approximately 1.9 and 2.0 times for $\mathrm{MA}_{20}$ and $\mathrm{A}_{20}$ films, respectively. Applying a simple Hertz model ${ }^{22}$ for the contact area between the heated probe and the flat polymer surface the increase in surface contact area was calculated as $\sim 1.8$ times. This is in good agreement with the increase in line widths obtained after $\mathrm{SThL}$ for both the $\mathrm{MA}_{20}$ as well as the $\mathrm{A}_{20}$ films for tip radii of 40 and $100 \mathrm{~nm}$, respectively. Ultimately, thinnest grooves written with $\operatorname{SThL}\left(r_{t i p} \sim 40 \mathrm{~nm}\right)$ in $\mathrm{MA}_{20}$ films were $83 \pm 7 \mathrm{~nm}$ in width. The relatively low activation energy for depolymerization of the $\mathrm{MA}_{20}$ film allowed the fast writing of patterns at speeds up to $500 \mu \mathrm{m} \mathrm{s}^{-1}$ and higher. In contrast, lines written this fast on $\mathrm{A}_{20}$ films did not show any surface change in topography and lateral force. Actually, lines written with SThL at $5 \mu \mathrm{m} \mathrm{s}^{-1}$ provided line widths of approximately $21 \mathrm{~nm}$ as was observed with lateral force microscopy. This was about half the size of the estimated tip radius used.

The optimized settings resulted in $r_{\text {tip }}$ to line width ratios of $\sim 0.5$ and $\sim 2.0$ for SThL on $\mathrm{MA}_{20}$ and $\mathrm{A}_{20}$, respectively. Cacialli and coworkers ${ }^{23}$ have reported on thermal chemical $\sim 30$ nanometer patterning of poly( $p$-phenylene vinylene) with a $5 \mu \mathrm{m}$ diameter platinum rhodium core bended wire tip with an estimated $100 \mathrm{~nm}$ contact radius. The achieved $r_{t i p}$ to line width ratio equals in this case approximately 3.6. This increase in pattern formation aspect ratio is related to the fact that the thermal probes as used throughout our experiments have a $12 \mu \mathrm{m} \times 12 \mu \mathrm{m}$ heater area positioned at the cantilever end above the tip. Therefore the heat source, although highly localized, is much more complex than the heated tip alone. Significant surface heating as described in Chapter 4 for example must be taken into account in order to achieve the highest possible resolution for SThL.

The effect of $r_{t i p}$ on the achievable resolution is also interesting in terms of tip wear. Typically, new tips have a radius in the order of $40 \mathrm{~nm}$ whereas this value readily increases to $100 \mathrm{~nm}$ after a few experiments. Hence tip wear must be taken into account for high resolution pattern reproducibility. Recently, King and coworkers $^{24}$ have adapted a method developed by Carpick and coworkers ${ }^{25}$ to fabricate wear resistant ultrananocrystalline diamond (UNCD) tips $\left(r_{\text {tip }} \sim 50 \mathrm{~nm}\right)$ at the end of their heatable silicon AFM cantilevers. Tip wear was significantly reduced (over a factor of 100) compared to silicon tips for relatively harsh writing conditions. Besides having improved wear resistance, the use of UNCD as tip material is beneficial for its' low surface energy reducing tip fouling, which is frequently 
observed for silicon tips to be the cause of increases in tip radius. A disadvantage of applying diamond tips is their limitation in tip temperatures, since diamond burns in air at temperatures higher than $600{ }^{\circ} \mathrm{C} .{ }^{26}$

In addition, carbon nanotubes (CNT) are also an interesting AFM probe tip material due to their high aspect ratio, typical sub $10 \mathrm{~nm}$ tip radius, and low tip wear. ${ }^{27}$ Hence SThL can possibly benefit from both the significantly reduced tip radius, allowing much smaller patterns to be produced as well as the long term tip radius stability. This was recognized by Vettiger and coworkers, ${ }^{28}$ who modified silicon heatable AFM probes with multi-walled CNT (MWCNT) tips. The approximately $150 \mathrm{~nm}$ to $350 \mathrm{~nm}$ long MWCNT tips were reported to have $r_{\text {tip }} \sim 10 \mathrm{~nm}$. The temperature decrease over the MWCNT is expected to be low due to the low thermal resistance of the tubes. Oxidative degradation of MWCNT tips occurred at temperatures (heater area) of $\sim 550{ }^{\circ} \mathrm{C}$. Hence similarly to diamond tips, CNT tips have a decreased upper temperature limit compared to silicon tips. Oxidative decomposition of the MWCNT tips resulted in shortening of the tips while $r_{\text {tip }}$ was practically unaffected.

In order to be able to achieve higher writing speeds for SThL films based on protected acrylates, we propose tuning the ester group to lower activation energies for faster thermolysis of the ester bond. In contrast, as we have shown for the fast depolymerization reaction, much higher writing speeds were obtained, with a penalty in achievable resolution for comparable tip temperatures. Hence an optimum is expected for the choice of ester group and writing speed, with respect to patterning resolution. Operating many probes parallel, in specially designed cantilever arrays (e.g. IBM Millipede $)^{29}$, enhances the pattering throughput with an order of 1000 or more. Future efforts should be made in these directions, in order to convert SThL as an attractive technique for lab scale prototyping of e.g. biosensors, into an established, widely applicable and commercially viable approach for large scale area patterning with sub 20 nanometer spatial resolution.

\subsection{Conclusions}

$\mathrm{MA}_{20}$ and $\mathrm{A}_{20}$ crosslinked polymer films with surface exposed tert-butyl ester protected carboxylic acid groups were prepared. TGA and FTIR spectroscopy measurements showed that for both $\mathrm{MA}_{20}$ and $\mathrm{A}_{20}$ thermal decomposition of the tert-butyl ester results in the loss of isobutylene yielding carboxylic acid groups, followed by the formation of intramolecular anhydride groups through the loss of water. $\mathrm{MA}_{20}$ initially showed depolymerization to be the primary reaction mechanism, whereas for $\mathrm{A}_{20}$ no depolymerization was observed. The apparent activation energy 
for tert-butyl ester decomposition was determined to be $125 \pm 13 \mathrm{~kJ} \mathrm{~mol}^{-1}$ and $116 \pm 7 \mathrm{~kJ} \mathrm{~mol}^{-1}$ for $\mathrm{MA}_{20}$ and $\mathrm{A}_{20}$, respectively. Both $\mathrm{MA}_{20}$ and $\mathrm{A}_{20}$ films showed improved thermomechanical stability during $\mathrm{SThL}$ compared to non crosslinked PS- $b$-P $t$ BA films. The availability of carboxylic acid moieties in subsequent wet chemical grafting reactions with amine functionalized fluorescent dyes following EDC/NHS chemistry was confirmed with (confocal) fluorescence microscopy. The apparent activation energy for groove formation in $\mathrm{MA}_{20}$ films with $\mathrm{SThL}$ was calculated to be $45 \pm 3 \mathrm{~kJ} \mathrm{~mol}^{-1}$. Lines prepared in $\mathrm{A}_{20}$ films were typically $\sim 10$ times less in width compared to lines written in $\mathrm{MA}_{20}$ films regardless of the tip radius of the heated probe used for SThL. This observation is ascribed to oxidative thermal depolymerization during $\mathrm{SThL}$ of $\mathrm{MA}_{20}$ films being the dominant reaction mechanism. Finally, the smallest line widths obtained for $\mathrm{MA}_{20}$ and $\mathrm{A}_{20}$ films with SThL were $83 \pm 7 \mathrm{~nm}$ and $21 \pm 2 \mathrm{~nm}$, respectively.

\subsection{Experimental}

Materials: Ethylene glycol dimethacrylate (EGDMA), tert-butyl methacrylate ( $t \mathrm{BMA})$, tert-butyl acrylate ( $t \mathrm{BA}), 1.4$ dibutanol diacrylate (dBDA), 1-ethyl-3-(3dimethylaminopropyl)carbodiimide hydrochloride (EDC), $N$-hydroxysuccinimide (NHS) and fluoresceinamine isomer I $\left(\lambda_{\max }=496 \mathrm{~nm}\right)$ were bought from Sigma Aldrich (St Louis, MO, USA). Ethanol was purchased from Biosolve (Valkenswaard, The Netherlands). Amine functionalized eFluor ${ }^{\circledR} 605 \mathrm{NC}\left(\lambda_{\mathrm{ex}}=350 \mathrm{~nm}-500 \mathrm{~nm}\right)$ CdSe core quantum dots were obtained from eBioscience, Inc. (San Diego, CA, USA) and Irgacure 184 (Ciba NV, Maastricht, The Netherlands) was a generous gift from ICH Zaanstad BV (Wormerveer, The Netherlands). Phosphate buffered saline solution (PBS, pH 7.4) was bought from B. Braun (Melsungen, Germany). Milli-Q water was produced by a Millipore Synergy system (Billireca, MA, USA). $\mathrm{PS}_{n}-b-\mathrm{P} t \mathrm{BA}_{m}$ (with degrees of polymerization $n$ and $m$ of 2092 and 1055, respectively) was purchased from Polymer Source Inc. (Dorval, Canada). Poly(isobutylene) $\left(\mathrm{M}_{\mathrm{n}} \sim 2900 \mathrm{~g} \mathrm{~mol}^{-1}\right.$ and PDI 1.23) was obtained from Polymer Standards Service GmbH (Mainz, Germany). All chemicals were used as received.

Film preparation: In a typical procedure, monomer mixtures comprising $80 \mathrm{wt} \%$ $t$ BMA and 20wt $\%$ EGDMA (for $\mathrm{MA}_{20}$ films) or $80 \mathrm{wt} \% t \mathrm{BA}$ and $20 \mathrm{wt} \% \mathrm{dBDA}$ (for $\mathrm{A}_{20}$ films) were prepared in a glass vial prior to polymerization. To these mixtures $3 \mathrm{wt} \%$ of Irgacure 184 as photo initiator was added. Subsequently the monomer mixture was purged with nitrogen. About $0.10 \mathrm{ml}$ of the monomer solution was deposited in the middle of a $26 \mathrm{~mm} \times 76 \mathrm{~mm}$ microscopy glass slide (Menzel-Glaser, Braunschweig, Germany) or a $\sim 20 \mathrm{~mm} \times 40 \mathrm{~mm}$ piece of silicon wafer (CZ, type $\mathrm{P}$, boron, $\langle 100 〉$, thickness $=525 \mu \mathrm{m}$, OKMETIC, Vantaa, Finland). Subsequently the 
solution was covered by placing a microscopy glass slide perpendicular orientated on top of the first slide/silicon support. The monomer mixture was allowed to spread between the two slides. Next the samples were UV irradiated by an array of 6 UV-B lamps (15 W, G15T8E, $\lambda=306 \mathrm{~nm}$, Ushio, Tokyo, Japan, sample to lamp distance $5 \mathrm{~cm}$ ) under nitrogen atmosphere for 30 minutes to polymerize and complete film formation. Careful separation of the bottom glass slide or silicon substrate yielded the polymer films on microscopy glass slides. The resulting film thickness was approximately $5 \mu \mathrm{m}$. Bulk samples were prepared according to the same procedure with that respect that aluminum differential scanning calorimetry pans (ALUM, Perkin Elmer, Waltham, MA, USA) were filled with the respective monomer solutions and covered by a glass microscopy slide. Glass slides were cleaned in piranha solution $\left(1: 3(\mathrm{v} / \mathrm{v})\right.$ solution of $30 \% \quad \mathrm{H}_{2} \mathrm{O}_{2}$ and concentrated $\left.\mathrm{H}_{2} \mathrm{SO}_{4}\right)$ for 10 minutes, rinsed with copious amounts of Milli-Q water and ethanol followed by spin drying, prior to use. Caution: Piranha solution should be handled with extreme caution. It has been reported to detonate unexpectedly. Silicon wafers were rinsed with ethanol and dried under a nitrogen stream, prior to use.

Fourier transform infrared (FTIR) spectroscopy: Single reflection attenuated total reflection (ATR) mode FTIR spectra (spectral resolution $4 \mathrm{~cm}-1,512$ scans) were collected with a Bruker model ALPHA FTIR spectrometer equipped with an ATR platinum diamond 1 reflection crystal (Bruker Optik GmbH, Ettlingen, Germany). Background spectra were recorded against air.

Thermo gravimetric analysis: The isothermal weight loss of bulk $\mathrm{MA}_{20}$ and $\mathrm{A}_{20}$ polymer samples $\left(\sim 8 \mathrm{mg}\right.$ ) under nitrogen (flow rate $20 \mathrm{ml} \mathrm{min}^{-1}$ ) was measured as a function of time for different temperatures with a Perkin-Elmer thermogravimetric analyzer (TGA 7, Waltham, MA, USA). The initial temperature ramp from room temperature to the thermolysis temperature was set to $200{ }^{\circ} \mathrm{C} \min ^{-1}$. The non isothermal weight loss of bulk polymerized EGDMA and dBDA samples $(\sim 8 \mathrm{mg})$ under nitrogen (flow rate $20 \mathrm{ml} \mathrm{min}{ }^{-1}$ ) was measured from $50{ }^{\circ} \mathrm{C}$ to $450{ }^{\circ} \mathrm{C}$ with a temperature ramp of $20^{\circ} \mathrm{C} \mathrm{min}^{-1}$.

FITR spectroscopy of TGA vapor reaction products: The vapor phase reaction products of $\mathrm{MA}_{20}$ and $\mathrm{A}_{20}$ samples isothermally heated at $260{ }^{\circ} \mathrm{C}$ in a thermogravimetric analyzer (nitrogen flow rate $20 \mathrm{ml} \mathrm{min}^{-1}$, TGA 7, Waltham, MA, USA) were fed to the inlet of an open gas cell positioned in a Vertex 70 FTIR spectrometer (Bruker Optik GmbH, Ettlingen, Germany) equipped with a MCT detector. The outlet of the gas cell was connected to a vacuum pump, maintaining a slight under pressure. FTIR spectra were continuously collected (spectral resolution $4 \mathrm{~cm}^{-1}, 4$ scans) for 10 minutes. For analysis over 30 spectra revealing the presence of reaction product were averaged. Hence the signal to noise ratio improved 
significantly. Background spectra (32 scans) were recorded against TGA outlet vapor for an empty sample pan isothermally heated to $260{ }^{\circ} \mathrm{C}$.

Scanning thermal lithography: Heatable probes (type AN-2, with $200 \mu \mathrm{m}$ long cantilevers, Anasys Instruments, Santa Barbara, CA, USA) mounted in a Dimension D3100 atomic force microscopy equipped with a hybrid scanner and a NanoScope IVa controller (Veeco/Digital Instruments (DI), Santa Barbara, CA, USA) were used for SThL. During the experiments the probe tip temperature was controlled with a Nano-TA2 controller (Anasys Instruments). Prior to the experiments, the probe tip temperature was calibrated using the following standard polymer materials: ${ }^{30}$ poly( $\varepsilon$-caprolactone) (PCL, $\left.T_{m}=55{ }^{\circ} \mathrm{C}\right)$, poly(ethylene) $\left(\mathrm{PE}, T_{m}=116^{\circ} \mathrm{C}\right.$ ) and poly(ethylene terephthalate) (PET, $T_{m}=235^{\circ} \mathrm{C}$ ). After SThL contact mode AFM images were taken with the same probes. Tapping mode AFM analysis was carried out with super sharp silicon cantilevers/tips (SSS-NCHR-10, super sharp silicon, typical tip radius of $\sim 2 \mathrm{~nm}$, Nanosensors, Wetzlar, Germany). The system operating frequency was typically $10 \%$ lower than the natural resonance frequency of the cantilever in air, the free amplitude was kept constant, while the set point amplitude was approximately $85 \%$ of the free amplitude $(\sim 1.5 \mathrm{~V})$.

Derivatization: Following SThL of polymer films the in situ formed carboxylic acid groups were activated with EDC/NHS (200 mM and $250 \mathrm{mM}$, respectively) in PBS. After 1 hour EDC/NHS activation the samples were rinsed 3 times with PBS and immersed in an amino functionalized eFluor ${ }^{\circledR} 605$ quantum dot solution in PBS (concentration $\sim 1 \times 10^{-6} \mathrm{M}$ ) for three hours, protected from direct sun light. The modified films were extensively rinsed with Milli-Q water prior to drying under a nitrogen stream and stored in the dark. Thermally activated bulk samples (Mettler FP82 hotstage temperature controlled by a Mettler FP80 central processor, MettlerToledo BV, Tiel, The Netherlands) were immersed in a fluoresceinamine solution in PBS (concentration $\sim 1 \times 10^{-4} \mathrm{M}$ ) for 1 hour, following EDC/NHS activation (200 $\mathrm{mM}$ and $250 \mathrm{mM}$, respectively in PBS, 1 hour). The fluoresceinamine functionalized samples were immersed in $50 \mathrm{ml}$ of Milli-Q water for 1 hour prior to rinsing with copious amounts of Milli-Q water and drying under a nitrogen stream.

(Confocal) fluorescence microscopy: Fluorescence microscopy images of dried fluoresceinamine modified bulk polymer samples were taken immediately after preparation of the samples with an Olypus IX71 fluorescence microscope. For the excitation of fluoresceinamine an U-MWB-2 filter cube was used, typical sample exposure times were $30 \mathrm{~ms}$. Confocal fluorescence microscopy images were taken with a Carl-Zeiss LSM510 confocal laser scanning microscope (Carl Zeiss MicroImaging GmbH, Göttingen, Germany) equipped with a LP 560 filter. For the excitation of eFluor ${ }^{\circledR} 605$ quantum dots an argon-ion laser with a wavelength of $488 \mathrm{~nm}$ was used. 


\subsection{References and notes}

1 a) Wouters, D.; Schubert, U. S. Angew. Chem. Int. Ed. 2004, 43, 2480. b) Krämer, S.; Fuierer, R. R.; Gorman, C. B. Chem. Rev. 2003, 103, 4367. c) García, R.; Martinez, R. V.; Martinez, J. Chem. Soc. Rev. 2006, 35, 29.

2 a) Wong, J. Y.; Leach, J. B.; Brown, X. Q. Surf. Sci. 2004, 570, 119. b) Lussi, J. W.; Michel, R.; Reviakine, I.; Falconnet, D.; Goessl, A.; Csucs, G.; Hubbell, J. A.; Textor, M. Prog. Surf. Sci. 2004, 76, 55. c) Mrksich, M. Chem. Soc. Rev. 2000, 29, 267.

3 a) Jonkheijm, P.; Weinrich, D.; Schröder, H.; Niemeyer, C. M.; Waldmann, H. Angew. Chem. Int. Ed. 2008, 47, 9618. b) Phizicky, E.; Bastiaens, P. I. H.; Zhu, H.; Snyder, M.; Fields, S. Nature 2003, 422, 208. c) MacBeath, G.; Schreiber, S. L. Science 2000, 289, 1760. d) Wells, M.; Crooks, R. M. J. Am. Chem. Soc. 1996, 118, 3988. e) LaFratta, C. N.; Walt, D. R. Chem. Rev. 2008, 108, 614 .

4 a) Mamin, H. J. Appl. Phys. Lett. 1996, 69, 433. b) Vettiger, P.; Despont, M.; Drechsler, U.; Dürig, U.; Häberle, W.; Lutwyche, M. I.; Rothuizen, H. E.; Stutz, R.; Widmer, R.; Binnig, G. K. IBM J. Res. Dev. 2000, 44, 323.

5 a) Christman, K. L.; Schopf, E.; Broyer, R. M.; Li, R. C.; Chen, Y.; Maynard, H. D. J. Am. Chem. Soc. 2009, 131, 521. b) A. A. Tseng, Small 2005, 1, 924.

6 a) Xia, Y. N.; Whitesides, G. M. Angew. Chem. Int. Ed. 1998, 37, 551. b) Weibel, D. B.; DiLuzio, W. R.; Whitesides, G. M. Nat. Rev. Microbiol. 2007, 5, 209.

7 a) Guo, L. J. J. Phys. D Appl. Phys. 2004, 37, R123. b) Chou, S. Y.; Krauss, P. R.; Renstrom, P. J. Science 1996, 272, 85. c) Chou, S. Y.; Krauss, P. R.; Renstrom, P. J. Appl. Phys. Lett. 1995, $67,3114$.

8 a) Böker, A.; Reihs, K.; Wang, J. G.; Stadler, R.; Ober, C. K. Macromolecules 2000, 33, 1310.

b) Böker, A.; Herweg, T.; Reihs, K. Macromolecules 2002, 35, 4929. c) La, Y. H.; Stoykovich, M. P.; Park, S. M.; Nealey, P. F. Chem. Mater. 2007, 19, 4538. d) La, Y. H.; Edwards, E. W.; Park, S. M.; Nealey, P. F. Nano Lett. 2005, 5, 1379.

Duvigneau, J.; Cornelissen, S.; Bardají Valls, N.; Schönherr, H.; Vancso, G. J. Adv. Funct. Mater. 2010, 20, 460.

10 Duvigneau, J.; Schönherr, H.; Vancso, G. J. Langmuir 2008, 24, 10825.

11 a) Feng, C. L.; Vancso, G. J.; Schönherr, H. Langmuir 2007, 23, 1131. b) Feng, C. L.; Vancso, G. J.; Schönherr, H. Langmuir 2005, 21, 2356. c) Feng, C. L.; Embrechts, A.; Vancso, G. J.; Schönherr, H. Eur. Polym. J. 2006, 42, 1954. d) Feng, C. L.; Embrechts, A.; Bredebusch, I.; Bouma, A.; Schnekenburger, J.; García-Parajó, M.; Domschke, W.; Vancso, G. J.; Schönherr, H. Eur. Polym. J. 2007, 43, 2177.

12 Szoszkiewicz, R.; Okada, T.; Jones, S. C.; Li, T. D.; King, W. P.; Marder, S. R.; Riedo, E. Nano Lett. 2007, 7, 1064.

13 Wang, D. B.; Kodali, V. K.; Underwood, W. D.; Jarvholm, J. E.; Okada, T.; Jones, S. C.; Rumi, M.; Dai, Z. T.; King, W. P.; Marder, S. R.; Curtis, J. E.; Riedo, E. Adv. Funct. Mater. 2009, 19, 3696.

14 Grassie, N. Pure Appl. Chem. 1972, 30, 119.

15 Grant, D. H.; Grassie, N. Polymer 1960, 1, 125.

16 a) Alfrey, T.; Haas, H. C.; Lewis, C. W. J. Am Chem. Soc. 1951, 73, 2851. b) Odian, G. Principles of Polymerization, 3rd ed.; John Wiley \& Sons Inc.: New York, 1991; p 692. 
17 FTIR spectroscopy of bulk polymerized EGDMA and dBDA samples before and after thermal decomposition at $250{ }^{\circ} \mathrm{C}$ for 10 minutes did not show thermal ester decomposition reactions of the crosslinkers.

18 a) Schaefgen, J. R.; Sarasohn, I. M. J. Polym. Sci. 1962, 58, 1049. b) Litmanovich, A. D.; Cherkezyan, V. O. Eur. Polym. J. 1984, 20, 1041.

19 a) Carpick, R. W.; Salmeron, M. Chem. Rev. 1997, 97, 1163. b) Morgenthaler, S. M.; Lee, S.; Spencer, N. D. Langmuir 2006, 22, 2706. c) Sasaki, K.; Koike, Y.; Azehara, H.; Hokari, H.; Fujihira, M. Appl. Phys. A 1998, 66, S1275.

20 Bykov, V.; Gologanov, A.; Shevyakov, V. Appl. Phys. A 1998, 66, 499.

21 Kashiwagi, T.; Hirata, T.; Brown, J. E. Macromolecules 1985, $18,131$.

22 Hertz, H. J. Reine Angew. Math. 1881, 92, 156.

23 Fenwick, O.; Bozec, L.; Credgington, D.; Hammiche, A.; Lazzerini, G. M.; Silberberg, Y. R.; Cacialli, F. Nature Nanotechnol. 2009, 4, 664.

24 Fletcher, P. C.; Felts, J. R.; Dai, Z. T.; Jacobs, T. D.; Zeng, H. J.; Lee, W.; Sheehan, P. E.; Carlisle, J. A.; Carpick, R. W.; King, W. P. ACS Nano 2010, 4, 3338.

25 Liu, J.; Grierson, D. S.; Moldovan, N.; Notbohm, J.; Li, S.; Jaroenapibal, P.; O'Connor, S. D.; Sumant, A. V.; Neelakantan, N.; Carlisle, J. A.; Turner, K. T.; Carpick, R. W. Small 2010, 6, 1140 .

26 Beffort, O.; Vaucher, S.; Khalid, F. A. Diam. Relat. Mater. 2004, 13, 1834.

27 a) Hafner, J. H.; Cheung, C. L.; Woolley, A. T.; Lieber, C. M. Prog. Biophys. Mol. Bio. 2001, 77, 73. b) Wilson, N. R.; Macpherson, J. V. Nature Nanotechnol. 2009, 4, 483.

28 Lantz, M. A.; Gotsmann, B.; Dürig, U. T.; Vettiger, P.; Nakayama, Y.; Shimizu, T.; Tokumoto, H. Appl. Phys. Lett. 2003, 83, 1266.

29 Binnig, G. K.; Cherubini, G.; Despont, M.; Dürig, U. T.; Eleftheriou, E.; Pozidis H.; Vettiger, P. In: B. Bhushan, Editor, Handbook of Nanotechnology, $2^{\text {nd }}$ ed., Springer Science and Business Media Inc., Heidelberg, 2006, p. 1457-1486.

30 In the calibration of the probe temperature using this procedure, the softening of the polymer substrates is determined. Since polymers do not possess a priori a defined melting point, this procedure introduced some errors, which, as we note explicitly, are not relevant for the work discussed here. Recent progress in calibration of the probe temperature has been reported by Abel et al.: Abel, M. R.; Wright, T. L.; King, W. P.; Graham, S. Compon. Packag. Technol. T. IEEE. 2007, 30, 200. See also Chapter 2. 



\section{Outlook}

This Thesis described our efforts to develop scanning thermal lithography (SThL) as a new concept for the spatially controlled highly localized thermochemical surface functionalization for the development of e.g. biosensors. We have demonstrated with the introduction of reactive imprint lithography (RIL) and SThL (Chapters 6 to 8) that heat can be exploited for the controlled thermal chemical surface functionalization across the length scales. Despite the demonstrated feasibility of SThL on soft condensed matter some challenges remain to be solved for this approach to reach a mature level.

For instance, the observed difference in thermolysis mechanism for the acrylate and methacrylate based polymer films, namely tert-butyl ester deprotection and poly(tert-butyl methacrylate) depolymerization, respectively, pointed towards an interesting parameter to control. The observed maximum line writing speed for these processes was $5 \mu \mathrm{m} \mathrm{s}^{-1}$ and $500 \mu \mathrm{m} \mathrm{s}^{-1}$, respectively. Hence, for lower apparent activation energy processes (i.e. in this case depolymerization) higher writing speed for SThL is possible. However the achieved minimum line width is broader compared to the higher activation energy process. This interplay between patterning speed and activation energy is directed towards the optimization of the choice for a protective group with respect to its activation energy for thermal deprotection. In addition, selecting a thermal labile protective group with a lower thermal deprotection temperature, lowers the probe tip temperatures that are required which might counterbalance the expected increase in resolution for selecting a lower activation energy thermal labile protective group.

In addition, with the currently available polymer films, only one type of chemical functionality is introduced at the surface of the polymer film after SThL, which lowers the applicability of the films for usage in large scale applications. King and coworkers ${ }^{1}$ have demonstrated that surface exposed primary amine groups that were thermally deprotected in subsequent SThL steps could be functionalized with multiple chemical functionalities following well known wet chemical grafting reactions. A disadvantage of this method is the multiple steps required to introduce for example three domains with different functionalities. Introduction of multiple protected functional groups with different thermal deprotection temperatures in the polymer films not only minimizes the number of processing steps required, it also makes them more attractive for large scale applications in e.g. biosensors. 
Furthermore we recognize the possibility to use sharper tips to enhance the resolution of the technique. For instance like described in Chapter 8, carbon nanotube functionalized tips, with tip radii of $\sim 10 \mathrm{~nm}$ are expected to enable SThL with a spatial resolution of $10 \mathrm{~nm}$ or less. Besides the use of carbon nanotube functionalized tips is beneficial in terms of the insignificant tip wear effects on the tip radius of curvature of carbon nanotube tips (see Chapter 2). This advantage was nicely described by Dürig and coworkers. ${ }^{2}$

SThL has been proven to have high potential for usage in future applications. For instance working cantilever arrays with over 5000 heatable cantilevers have been reported. ${ }^{3}$ In addition, the fundamental limit in writing speed due to heat transport at the probe tip-polymer contact point has been calculated to be on the order of $\sim 30 \mathrm{~mm} \mathrm{~s}^{-1}$. Hence, the suggested directions for future research mainly aim at improvement of the polymer film platforms for SThL. These directions offer in our opinion the best opportunities for increasing the number of embedded chemical functionalities and enhancing the patterning throughput.

\section{References}

1 Wang, D. B.; Kodali, V. K.; Underwood, W. D.; Jarvholm, J. E.; Okada, T.; Jones, S. C.; Rumi, M.; Dai, Z. T.; King, W. P.; Marder, S. R.; Curtis, J. E.; Riedo, E. Adv. Funct. Mater. 2009, 19, 3696.

2 Lantz, M. A.; Gotsmann, B.; Dürig, U. T.; Vettiger, P.; Nakayama, Y.; Shimizu, T.; Tokumoto, H. Appl. Phys. Lett. 2003, 83, 1266.

3 Binnig, G. K.; Cherubini, G.; Despont, M.; Dürig, U. T.; Eleftheriou, E.; Pozidis H.; Vettiger, P. In: B. Bhushan, Editor, Handbook of Nanotechnology, $2^{\text {nd }}$ ed., Springer Science and Business Media Inc., Heidelberg, 2006, p. 1457-1486.

4 Szoszkiewicz, R.; Okada, T.; Jones, S. C.; Li, T. D.; King, W. P.; Marder, S. R.; Riedo, E. Nano Lett. 2007, 7, 1064. 


\section{Summary}

The aim of this Thesis is the development of scanning thermal lithography (SThL) as an alternative nanotechnology tool for scanning probe lithography (SPL) of soft condensed matter at the sub 50 nanometer length scale. Heated atomic force microscopy (AFM) cantilevers are used as spatially controlled highly localized heat sources for the thermochemical modification of tert-butyl (meth)acrylate based polymer film platforms. The surface exposed tert-butyl esters of these polymer platforms are known to be thermally cleavable to yield carboxylic acid moieties at the air exposed interface that are readily available for subsequent wet chemical grafting reactions with relevant (bio)molecules. The length scales of heat transport, as well as effects from the proximity of solid substrates supporting the polymer films on the heated probe tip interface temperature, are discussed in detail. In addition, a systematic study on the effect of copolymer film composition on the resolution of SThL is presented, starting from poly(styrene)-block-poly(tert-butyl acrylate) (PS- $b$-P $t$ BA) films, followed by specifically tailored tert-butyl ester exposing reactive surfaces.

A short introduction to the topics discussed in this Thesis and the motivation for the research described herein are provided in Chapter 1.

In Chapter 2 a literature overview on the general topics discussed in this Thesis is presented. Cantilever design, probe tip temperature calibration and an overview of scanning thermal microscopy applications are highlighted.

In Chapter 3 transient heat flow effects for a heated cantilever-tip assembly in contact with a polymer film are described. The heated probe-induced surface crystallization of amorphous poly(ethylene terephthalate) (PET) films revealed a steep short range temperature gradient close to the tip-polymer contact interface. In addition, thermal expansion measurements in poly(dimethylsiloxane) (PDMS) films with varying thickness values provided insight in the long range thermal transport during typical non steady state nanoscale thermal analysis (NanoTA) conditions. The results obtained emphasize the importance of taking heat transport from the heated cantilevers in contact with a polymer film to the environment into account for optimizing the resolution of SThL.

In Chapter 4 heated probe tip-sample interface temperature $\left(T_{i}\right)$ deviations from the calibrated $T_{i}$ for NanoTA measurements on thin polymer films are described. With decreasing film thickness an increase in measured softening temperature by NanoTA was observed for films on good thermally conductive solid supports (e.g. silicon or glass). This effect was more pronounced for silicon compared to glass, which is 
ascribed to the $\sim 100$ times higher thermal conductivity of silicon compared to glass. The close proximity of the highly thermally conductive solid support resulted in effective lowering of $T_{i}$. The deviation of $T_{i}$ from the calibrated $T_{i}$ was several tens of degrees Celsius for $50 \mathrm{~nm}$ thick poly(styrene) (PS), polycarbonate (PC) and poly(methyl methacrylate) (PMMA) films on silicon substrates. Significant silicon substrate proximity effects occurred for polymer film thicknesses below several hundreds of nanometers.

In Chapter 5 the melting behavior of poly(isoprene)-blockpoly(ferrocenyldimethylsilane) (PI- $b$-PFS) crystals deposited on hydrophilic (native oxide surface of silicon) and hydrophobic (highly ordered pyrolytic graphite) surfaces was described. Following an in situ isothermal temperature controlled AFM study, the heatable AFM cantilevers were introduced as a novel tool for the rapid nanoscale thermal analysis of the PI- $b$-PFS crystalline architectures. NanoTA measurements on the 15 nanometer thick PI- $b$-PFS crystals deposited on silicon were found to be below the physical limit of the technique. This was ascribed to $i$ ) the low sensitivity of the heated cantilevers with respect to the small sample penetration of thin samples and ii) the decreased probe tip interface temperature in close proximity of the silicon substrate. The latter effect is in full agreement with the results presented in Chapter 3 and Chapter 4.

Reactive imprint lithography (RIL) was introduced in Chapter 6 as a new onestep approach for the combined thermal chemical surface functionalization and topographical patterning of PS- $b$-P $t$ BA block copolymer films. Imprinting above the tert-butyl ester deprotection temperature yielded topographically patterned polymer films with carboxylic acid functional groups exposed at the surface. Well established wet chemical grafting reactions were used to confirm the availability of the formed carboxylic acid groups for bioconjugation. Besides the introduction of RIL as an interesting complementary approach for the preparation of reactive (bio)interfaces in a one-step process, the feasibility of thermal chemical surface functionalization of tert-butyl ester protected acrylates was also demonstrated. Hence Chapter 6 formed one of the pillars for an in depth investigation of exploiting SThL on tert-butyl ester containing polymer film platforms.

Following the successful large area thermal chemical surface functionalization with RIL, Chapter 7 continues with exploring SThL for the localized spatially controlled thermochemical surface functionalization of PS- $b$-P $t$ BA films. First the thermolysis kinetics and thermal deprotection mechanism of PS- $b$-P $t$ BA were established followed by the introduction of SThL. The smallest domain sizes obtained remained above the critical sub $100 \mathrm{~nm}$ length scale, which was ascribed to the poor thermomechanical properties of the block copolymer films at the relatively high temperatures used during SThL $\left(\sim 250^{\circ} \mathrm{C}\right)$. Hence the formed domains are the result 
of thermomechanical surface deformation combined with the localized thermochemical deprotection of the $\mathrm{P} t \mathrm{BA}$ block.

Therefore in Chapter $\boldsymbol{8}$ the development of crosslinked tert-butyl ester protected carboxylic acid groups containing (meth)acrylate based copolymer substrates for $\mathrm{SThL}$ is described. Crosslinking the polymer films enabled the development of thermochemical SThL that does not result in thermomechanical surface deformation. The difference in thermal deprotection mechanism for methacrylate and acrylate based films (depolymerization versus ester deprotection, respectively) was discussed in detail. The higher apparent activation energy $\left(E_{a}\right)$ observed for tert-butyl ester deprotection in the tert-butyl acrylate system compared to the $E_{a}$ for poly(tert-butyl methacrylate) depolymerization significantly enhances the resolution of thermochemical surface functionalization via SThL. The chemical functionality of the deprotected tert-butyl acrylate domains (i.e. acrylic acid domains) was confirmed by fluorescence microscopy following wet chemical derivatization with a fluorescent dye. The highest achieved resolution obtained with SThL on the crosslinked tert-butyl acrylate based films is approximately $20 \mathrm{~nm}$, which is below the radius of curvature of the used probes.

The work described in this Thesis contributes significantly to the development of SThL. Deepened insights in the thermal transport routes as well as in probe tip-sample interface temperatures for heated cantilevers in contact with a polymer film were obtained. Differences in thermal deprotection mechanism for tert-butyl methacrylate and tert-butyl acrylate based polymer film platforms emphasize the importance of understanding the thermal deprotection mechanism in detail in order to achieve the highest possible resolutions for SThL. Future directions envisaged to enhance the further development SThL should focus on the incorporation of multiple functionalities within the polymer film platforms as well as on increasing the patterning throughput. In addition, sharper probe tips with tip radii of curvature smaller than 30 nanometer (e.g. carbon nanotube tips) compared to the commercially available cantilevers used throughout this Thesis, are expected to further enhance the resolution. 



\section{Samenvatting}

Het doel van het werk beschreven in dit proefschrift is de ontwikkeling van scanning thermal lithography (SThL) als een alternatieve nanotechnologie toepassing voor scanning probe lithography (SPL) van polymere materialen op sub-50 nanometer lengteschaal. Verwarmde atomic force microscopy (AFM) tips worden gebruikt als nauwkeurig te positioneren, zeer locale warmte bronnen voor de thermochemische modificatie van tert-butyl (meth)acrylaat gebaseerde polymeer film platformen. De zich aan het oppervlak bevindende tert-butyl esters van deze polymeer platformen kunnen thermisch worden afgesplitst, wat resulteert in de vorming van carbonzuurgroepen in het aan lucht blootgestelde grensvlak. De aldus gevormde carbonzuurgroepen zijn zeer geschikt voor chemische vervolg reacties met relevante (bio)moleculen. De lengteschalen van warmtetransport, alsmede het effect van de nabije aanwezigheid van polymeer film ondersteunende substraten op de verwarmde probe tip grensvlak temperatuur, worden in detail besproken. Daarnaast wordt een systematische studie naar het effect van de copolymeer film compositie op de resolutie van SThL gegeven, met als uitgangspunt poly(styreen)-blok-poly(tert-butyl acrylaat) (PS- $b$-P $t$ BA) films, gevolgd door speciaal ontworpen tert-butyl ester bevattende reactieve oppervlakken.

Een korte inleiding van de onderwerpen besproken in dit proefschrift en de motivatie voor het hierin beschreven uitgevoerde onderzoek wordt gegeven in Hoofdstuk 1.

In Hoofdstuk 2 wordt een literatuuroverzicht van de algemene onderwerpen besproken in dit proefschrift gegeven. Probe ontwerp, probe tip temperatuur kalibratie en een overzicht van scanning thermal microscopy worden met name toegelicht.

In Hoofdstuk 3 worden niet evenwichts warmtetransport effecten voor een verwarmde probe in contact met een polymeer film beschreven. De verwarmde probe tip geïnduceerde oppervlakte kristallisatie van amorfe poly(ethylene terephthalate) (PET) films onthulde een steile temperatuurgradiënt nabij het tip-polymeer contact punt. Daarnaast gaven thermische uitzettingsmetingen op poly(dimethylsiloxaan) (PDMS) films met een variërende dikte inzicht in warmtetransport op grotere lengteschalen tijdens nanoscale thermal analysis (NanoTA) metingen met representatieve non steady state condities. De verkregen resultaten onderstrepen dat warmtetransport, van de verwarmde probes in contact met een polymeer film naar de omgeving toe, moet worden meegenomen in de optimalisatie van de resolutie voor SThL.

In Hoofdstuk 4 wordt de afwijking van de verwarmde probe tip-sample grensvlak temperatuur $\left(T_{i}\right)$ van de gekalibreerde $T_{i}$ voor NanoTA metingen op dunne polymeer 
films beschreven. Voor afnemende filmdiktes werd een toename in de verwekingstemperatuur gemeten met NanoTA voor films op substraten met een goede warmtegeleiding (bijvoorbeeld silicium of glas). Dit effect was groter voor silicium in vergelijking met glas, wat is toegeschreven aan de ongeveer 100 keer hogere warmtegeleiding van silicium ten opzichte van glas. De nabije aanwezigheid van de goed warmte geleidende substraten resulteerde in het effectief verlagen van $T_{i}$. De afwijking van $T_{i}$ ten opzichte van de gekalibreerde $T_{i}$ was in de orde van enkele tientallen graden Celsius voor $50 \mathrm{~nm}$ dikke poly(styreen) (PS), polycarbonaat (PC) en poly(methyl methacrylaat) (PMMA) films op silicium substraten. Significante afwijkingen van $T_{i}$ door de nabije aanwezigheid van goede warmte geleidende substraten werd al waargenomen voor films dunner dan enkele honderden nanometers.

In Hoofdstuk 5 wordt het smelt gedrag van poly(isopreen)-blok-poly(ferrocenyl dimethylsilaan) (PI- $b$-PFS) kristallen op hydrofiele (silicium oxide) en hydrofobe (highly ordered pyrolytic graphite) oppervlakken beschreven. Volgend op de in situ isothermische, temperatuur gecontroleerde AFM studie worden de verwarmbare AFM probes geïntroduceerd als een nieuwe techniek voor de snelle op nanoschaal uit te voeren thermische analyse van de PI-b-PFS kristallijne architecturen. NanoTA metingen op de 15 nanometer dikke PI- $b$-PFS kristallen gedeponeerd op silicium bleken beneden de fysische limiet van de techniek te zijn. Dit werd toegeschreven aan i) de lage gevoeligheid van de verwarmde probes in relatie tot de lage sample penetratie diepte van deze dunne samples en $i$ ) de verlaagde probe tip grensvlak temperatuur door de nabije aanwezigheid van het silicium substraat. Dit laatste effect is in goede overeenstemming met de resultaten gepresenteerd in Hoofdstuk 3 en

\section{Hoofdstuk 4.}

Reactive imprint lithography (RIL) werd geïntroduceerd in Hoofdstuk 6 als een nieuwe één staps benadering voor de gecombineerde thermisch chemische oppervlakte functionalisatie en topografische patroonoverdracht van PS- $b$-P $t$ BA blok copolymeer films. Imprinting boven de tert-butyl ester ontschermingstemperatuur resulteert in topografisch vormgegeven polymeer films met carbonzuur functionaliteit aan het lucht grensvlak. Bekende waterige koppelings reacties werden toegepast om de beschikbaarheid van de carbonzuurgroepen aan het oppervlak voor bioconjugatie aan te tonen. Naast de introductie van RIL als een interessante complementerende techniek voor de vervaardiging van reactieve (bio)grensvlakken in een stap, werd ook de haalbaarheid van thermisch chemische oppervlakte functionalisatie van tert-butyl ester beschermde acrylaten gedemonstreerd. Hierdoor vormt Hoofdstuk 6 een van de pijlers voor de uitvoerige studie naar de toepassing van SThL op tert-butyl ester bevattende polymeer film platformen.

Volgend op de succesvolle thermisch chemische grensvlak functionalisatie van grote oppervlakken door middel van RIL, gaat Hoofdstuk 7 verder met de verkenning 
van de toepasbaarheid van SThL op PS- $b$-P $t$ BA films. Allereerst werden de thermolyse kinetiek en het thermische ontschermingsmechanisme van PS- $b$-P $t$ BA vastgesteld, gevolgd door de introductie van SThL. De kleinste gefunctionaliseerde domeinen bleven boven de kritische sub $100 \mathrm{~nm}$ lengteschaal. Dit werd toegeschreven aan de slechte thermisch mechanische eigenschappen van de blok copolymeer films bij de relatief hoge temperaturen gebruikt tijdens SThL $\left(\sim 250{ }^{\circ} \mathrm{C}\right)$. De aldus gevormde domeinen zijn hierdoor het resultaat van thermisch mechanische oppervlakte deformatie gecombineerd met de lokale thermisch chemische ontscherming van het $\mathrm{P} t \mathrm{BA}$ blok.

Als gevolg hiervan wordt in Hoofdstuk 8 de ontwikkeling van gecrosslinkte tertbutyl ester beschermde carbonzuurgroepen-bevattende (meth)acrylaat-gebaseerde copolymeer substraten voor SThL beschreven. Crosslinken van de polymeer films resulteerde in de ontwikkeling van thermisch chemische SThL zonder thermisch mechanische oppervlakte deformatie. Het verschil in thermisch ontschermingsmechanisme voor methacrylaat en acrylaat gebaseerde films (respectievelijk depolymerisatie versus ester ontscherming) wordt uitvoerig besproken. De waargenomen hogere klaarblijkelijke activeringsenergie $\left(E_{a}\right)$ voor tert-butyl ester ontscherming in het tert-butyl acrylaat gebaseerde systeem vergeleken met de $E_{a}$ voor depolymerisatie van poly(tert-butyl methacrylaat) resulteerde in een significante verbetering van de behaalde resolutie voor de thermisch chemische oppervlakte functionalisatie met SThL. De chemische functionaliteit van de ontschermde tert-butyl acrylaat domeinen (ofwel acrylzuur domeinen) werd aangetoond met fluorescentie microscopie na chemische koppeling van fluorescente moleculen. De hoogst behaalde resolutie met SThL op het gecrosslinkte tert-butyl acrylaat gebaseerde systeem is ongeveer $20 \mathrm{~nm}$. Dit is beneden de tip radius van de gebruikte probes.

Het onderzoek beschreven in dit Proefschrift draagt significant bij aan de ontwikkeling van SThL. Diepgaand inzicht werd verkregen in de thermische transport routes alsmede in $T_{i}$ voor verwarmde probes in contact met polymeer films. Waargenomen verschillen in de thermische ontschermingsmechanismen voor tert-butyl methacrylaat en tert-butyl acrylaat gebaseerde polymeer film platformen benadrukken dat gedetailleerde kennis van de thermische ontschermingsreactie mechanismen onontbeerlijk is voor het uitvoeren van SThL met de best haalbare resolutie. Voor de verdere ontwikkeling van SThL worden de uitbreiding van het aantal chemische functionaliteiten geïncorporeerd in de polymeer film platformen alsmede de doorvoercapaciteit genoemd. Daarnaast wordt de ontwikkeling van probe tips met een tip radius kleiner dan 30 nanometer (bijvoorbeeld carbon nanotube tips) aangeraden om de resolutie van SThL verder te verbeteren. 



\section{Acknowledgements}

Having finished the scientific part of my Thesis I would like to use a few more pages to express my deepest gratitude to all those that supported me from the start to the end. And what a start it was.... Since then I envisaged many times to come this far, as an internal motor it kept me going. This motor was refueled numerous times by the wonderful people that surrounded me during these years. Without them the work described in this Thesis would not have been accomplished.

First of all I would like to acknowledge my promoter, Prof. Julius Vancso, who offered me the opportunity to be part of MTP. Dear Julius, thank you very much for the great support and freedom you provided me to perform my own research. You, as an 'old school physicist', taught me, 'a new age engineer', how to refocus my eyes on the fields of science and technology from a different point of view. This was one of the main reasons I wanted to join your group. I really enjoyed my stay in your group.

The second person I would like to express my deepest gratitude to is my assistant promoter, Prof. Holger Schönherr. Dear Holger, thank you very much for your guidance and endless support. You were actually the second reason I wanted to join MTP. Your skills to motivate, teach and guide people are one of a kind. I never left empty handed your office. Frequently, improved experiments or data analysis were directly the result of a scientific discussion with you. I sincerely appreciate it that you were always there to reach, even after you left to Siegen.

Dear Julius and Holger, I would like to thank both of you for your confidence in me during the start of the project. I will never forget how both of you allowed me to recover and how sometimes you had to protect me for my own drive to rush.

I am grateful to dr. Mark Hempenius for his support in the chemical lab and your sense of humor that surfaced after I got to know you better. I also thank you for correcting my Thesis. I would like to thank Geneviève Rietveld (Gen) for her help with the endless paperwork and the many small talks we had. I am grateful for your efforts to design my cover. The next person I would like to thank is dr. Peter Schön. Dear Peter, soon after you arrived to Twente our brainwaves were in phase and we were capable to discuss many things, both scientific matters as well as other less defined topics. I really enjoyed our trip to Boston, SIR, YES, SIR.... WAIT.... I also would like to thank you for correcting my Thesis.

The next person to be grateful to is mentioned in so many acknowledgements that if I would sum up the 'special words of thank you' addressed to him, I could write another chapter. Dear Clemens, without you taking care of everything, the life of 
MTP members would be more harsh. You have the incredible ability to fix equipment that appears to be FUBAR and you are always willing to help out with anything. The many breaks we had together were filled with among others, lots of fun, serious talk, construction advise and way too much coffee. Clemens, thank you for your friendship and for one of the cupboards that you emptied (or are about to empty).

During the past couple of years I had the pleasure to work with three students, Stijn Cornelissen, Núria Bardají Valls and Michel Klein Gunnewiek. Their work contributed significantly to Chapters 3 and 6 of my Thesis, for which they are acknowledged. Dear Stijn, your clear understanding of science and outstanding organization skills will definitely contribute to your success as entrepreneur (I already see you are building an empire). Dearest Núria, The Kidd, the time we spend together during your stay in '399' I enjoyed a lot. I am sure you will find your way with the synthesis you are struggling with these days ;-) and I promise a visit to Barcelona is on the time table. Such a small lady with a very big heart! I will never forget you. Last but not least there is this rider of the apocalypse. Dear Michel, your devoted contributions to mastering the evil beasts have complemented my work significantly. Your independent style of working and tackling problems will be beneficial for you for the coming four years. I am looking forward to our renewed carpooling sessions.

Now it is time to thank my two office mates, Edit Kutnyanszky and Qi Chen for their support, corrections on my chapters and their endurance (I tend to speak when I think.... and thus I talk a lot....). It is my pleasure and honor to have both of you as my paranimfen. Dear Edit, you were always there willing to help out with anything or to talk about life, science and more. Fortunately our daily conversations during the early morning coffee will continue for a while. We sure had some good drinking parties, in which neither of us was willing to resist one more last.... Dear Qi, my only and thus favorite matching, together we managed the bureaucracy of the system. Thank you for addressing the so many small questions, with patience and a smile. Besides the interesting scientific struggles we discussed, our talks on a personal level were appreciated no less. I thank you both for being the best officemates.

Next I would like to thank all present and former MTP group members I had the chance to work and spend time with. Janet, your generous hospitality and taking care of everyone is incredible. I really enjoyed the numerous talks we had about whatever was to our minds. Denis, best representative of Russia, I am glad your sense of humor was international minded. Eddy, I still miss your remarkable ability to give daily words or phrases a totally new dimension. Dear Yujie, as a part of the Chen family, you also have a special place in my memories. I appreciated the many talks we had and your open attitude to my western style of communication. With you, Qi and Walter (your adorable son), there was always fun. Anika, I thank you for the many (scientific) discussions we had, the drinking sessions and your support during these 
years. I would also like to thank: Oya (I hope once you will understand guys, my advice is still free of charge), Aysegul, XiaoFeng (you are an amazing guy), Wilma (thanks a lot for the corrections), Monique, Marina \& Miguel, In Yee (it was always fun to work (and drink) with you) \& Xing Yi, Cynthia, Melba, Hong-Jing, Azis, Matthijs, Mine, Gabriella, Nikodem, Szczepan (thanks for the scientific support), Xueling, Nayeli, Gerwin, Anna (thanks for the little cows), Saurabh, Kristin, Joris, Johannes, Weiqing (weightwatcher, but remember size matters), Thomas, Maria, Emilia, Ewa, Nina, Yuan Yuan, German Alex (womanizer) and last but not least the other three riders of the apocalypse: Bas, Fons and Lennard. In addition, I would like to thank a few more people from other groups: Jacob (thanks for the rides and your great anecdotes) and Mukund (I thank you for your friendship and good times we had), Hans, Sytze, Martin, Niels, Janine, Mark Ankone, Jeleamy (I wish your tongue will heal), ShuHan Hsuuuuuuuuu (I can still hear you), Richard and Marcel, Jordi, Franscesca, Pieter, Kim (you can't stop loving pink), Michiel (some NanoNed conferences had good (after) parties), Bernke and Maureen. I apologize if I forgot someone. Thanks to all of you for the good time we had.

Daarnaast wil ik graag mijn 'hometown' vrienden en familie bedanken voor de getoonde interesse in mijn werk en voor de steun en toeverlaat. Mijn 'Brother in Arms', Jelle, uniek als je bent, heb je me vaak verbaasd doen staan hoe simpel het is wat druk af te laten en alles weer te relativeren.

Alle hierboven genoemde personen hebben er toe bijgedragen dat ik met veel plezier en motivatie mijn onderzoek heb uitgevoerd. Maar ik zou niet zover zijn gekomen zonder mijn naaste familie. Allereerst wil ik graag Ellie, Joris en Bart bedanken voor het warme hart dat ze me toedragen. Freek en Corine, jullie als broer en 'zus' hebben me altijd gesteund en interesse getoond in mijn werk. Te weten dat je op elkaar kunt bouwen is heel waardevol. Mijn diepste respect gaat uit naar mijn ouders, Ada en Marcel, jullie hebben altijd voor mij klaar gestaan en jullie interesse en vertrouwen in mij hebben me gevormd tot wie ik nu ben. Een voorbeeld dat ik slechts hoop te kunnen evenaren. Lieve Ellen, zonder jouw onvoorwaardelijke support had ik dit niet kunnen realiseren. Met het leven dat in je groeit zullen we nog een droom waar maken. Ik heb je lief, nu en in de toekomst.

Joost 



\section{Curriculum Vitae}

Joost Duvigneau was born on May $11^{\text {th }}, 1981$ in Winterswijk, The Netherlands. After graduating from secondary school (O.S.G. De Driemark, Winterswijk), he continued his studies at the University of Twente. He studied chemical engineering with a major in biomedical material engineering. The master research was performed in the group of Professor Jan Feijen on the development of block copolymer based targeted drug delivery systems. After graduating in 2005, he started pursuing a Ph.D. degree under the supervision of Professor G. Julius Vancso at the University of Twente. The results of his research are described in this Thesis. Since January $1^{\text {st }} 2011$, he has been appointed as CTO of Aerotech Development B.V. (Heteren, The Netherlands). 
